\title{
TECHNICAL \\ DEVELOPMENT of \\ INTERVENTIONAL \\ NUCLEAR IMAGING
}

Martijn M.A. Dietze 


\section{Colophon}

Technical Development of Interventional Nuclear Imaging

Dissertation, Utrecht University - with a summary in Dutch

(C) Martijn M.A. Dietze, Utrecht, 2020

Publication of this dissertation was financially supported by:

Nuclear Fields

Quirem Medical

Sirtex Medical

ChipSoft

All rights reserved. No part of this publication may be reproduced or transmitted in any form or by any means without permission in writing from the author. The copyright of the articles that have been published or have been accepted for publication has been transferred to the respective journals.

ISBN:

$978-90-393-7315-6$ 


\section{Technical Development of Interventional Nuclear Imaging}

Technische Ontwikkeling van Interventionele Nucleaire Beeldvorming (met een samenvatting in het Nederlands)

\section{Proefschrift}

ter verkrijging van de graad van doctor aan de Universiteit Utrecht op gezag van de rector magnificus, prof. dr. H.R.B.M. Kummeling, ingevolge het besluit van het college voor promoties in het openbaar te verdedigen op donderdag 17 september des ochtends te 11.00 uur

door

Martijn Michiel Alexander Dietze

geboren op 18 september 1993

te Capelle aan den IJssel 
Promotores: $\quad$ Prof. dr. ir. H.W.A.M. de Jong

Prof. dr. ir. M.A. Viergever

Prof. dr. M.G.E.H. Lam 


\section{Contents}

Chapter 1

Introduction

Chapter 2

A Prototype Interventional Scanner

Chapter 3

A Full-Scale Interventional Scanner

Chapter 4

Nuclear Imaging with an X-Ray Flat Panel Detector

Chapter 5

Respiratory Motion Compensation for Interventional Imaging

Chapter 6

Interventional Respiratory Motion Compensation Validation

Chapter 7

Nuclear Imaging with Focusing Collimators

Chapter 8

Multifocal Collimator Imaging Validation

Chapter 9

An Adaptive Nuclear Scan Duration

Chapter 10

Accelerated Image Reconstruction with Deep Learning

Chapter 11

Summary and Discussion

Addenda

Nederlandse Samenvatting

Acknowledgments

List of Publications

Biography 


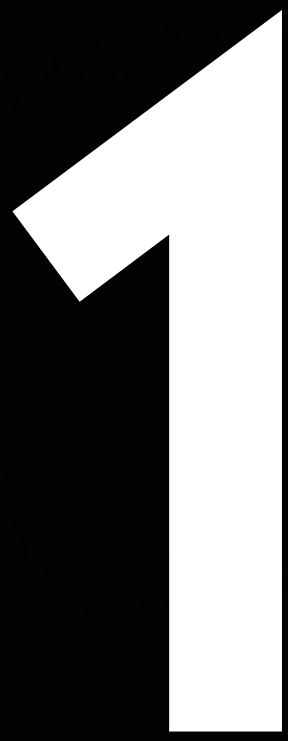


INTRODUCTION 


\section{Diagnostic Imaging}

Imaging has an important role in medicine by supporting disease diagnosis. Examples of imaging modalities that are often employed to visualize a patient's (patho)physiological anatomy include magnetic resonance imaging (MRI), computed tomography (CT), and ultrasound (US) imaging [1]. A medical imaging technique that focusses more specifically on the (dys)functioning of the tissue rather than its anatomy, is nuclear imaging [2].

\section{Nuclear Imaging}

Nuclear imaging is performed by the administration of radiopharmaceuticals that accumulate in specific locations in the body. Different radiopharmaceuticals are used for different purposes [3]. When tumors need to be visualized, a radiopharmaceutical can be administered that accumulates in tissues with increased metabolic activity [4]. When one wants to evaluate thyroid functioning, iodine isotopes can be injected since these replace the endogenous molecules in the thyroid with their radioactive counterparts [5]. The radionuclides that are used for such diagnostic purposes emit photons ( $\gamma$-radiation) that are not harmful to the patient at low activity levels.

The photons emitting from the radionuclides radiate out of the body in all directions. By scanning the patient on a dedicated device that captures these photons, the radionuclide distribution in the body can be visualized and a clinical evaluation can be made. Two imaging procedures are currently used in nuclear medicine to visualize radionuclide distributions: single-photon emission computed tomography (SPECT) and positron emission tomography (PET) [6-9], which rely on different physical principles for their photon detection. This dissertation will solely investigate imaging using the SPECT scanner (Fig. 1).

\section{SPECT Scanner}

The SPECT scanner consists of one or multiple gamma cameras for the detection of the gamma photons. These gamma cameras compromise a collimator, a scintillator, and photomultipliertubes [10]. The collimator is made out of a dense material (usually, lead) that has an alternating pattern of holes and septa [11]. Only the photons that are traveling in the direction parallel to the septa can pass through the holes in the collimator so that directional information is acquired. These photons then interact with a scintillation material (often, thallium-doped sodium iodine) that transforms the photons into light. The signal arising from a single photon is low and should be amplified. This is achieved by the photomultiplier tubes that convert the light into an electronic signal that can be measured. The strength of the electronic signal is proportional to the energy of the gamma photon and the signal distribution across multiple photomultiplier tubes can be used to determine the initial gamma photon interaction position.

It can be challenging to determine where exactly in the nuclear images the radionuclide accumulations occurred since no detailed anatomical information is given. An important innovation in nuclear medicine has hence been the introduction of hybrid imaging systems that consist of SPECT 


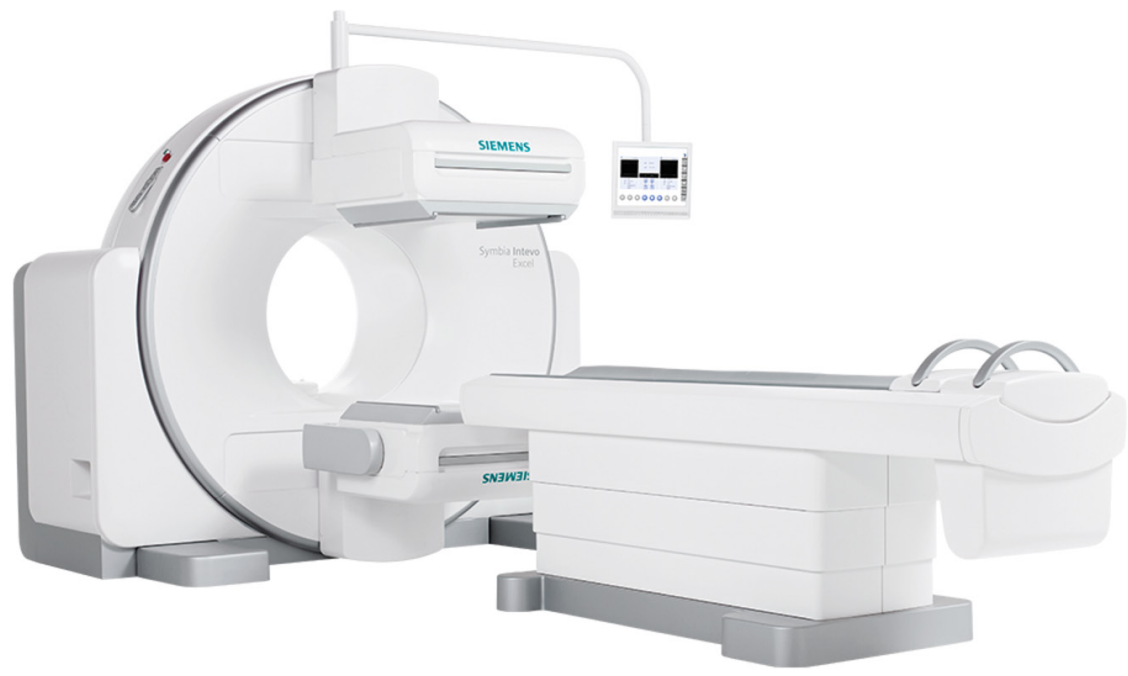

Figure 1: A typical example of a hybrid clinical SPECT/CT scanner (displayed is the Siemens Intevo Excel; courtesy of Siemens Healthineers).

scanners that are coupled to CT scanners so that such anatomical information is included (see Fig. 2) [12]. These SPECT/CT scanners acquire the images in sequence: the CT scan is made first and, while the patient is still on the table, a SPECT scan is acquired next. The combination of several modalities into one system has proved to be very valuable and has substantially increased the presence of nuclear imaging in the imaging workflow.

\section{Image Acquisition}

Planar images (2D) are acquired when the patient is scanned with a non-moving detector. It is, however, often beneficial to visualize a distribution in 3D since this allows a more detailed evaluation of the radionuclide distribution. Such 3D imaging can be achieved by rotating the detector around the patient to obtain images over many different angles. All of these images should then be combined to determine the source distribution. This is a mathematical inverse problem that is known as image reconstruction and many approaches (analytical and iterative) have been developed to approximate its solution [13-15].

SPECT scans generally require a substantial acquisition time ( $>20 \mathrm{~min}$ ) to gather sufficient photon events and are hence not performed under breath-hold (as is commonly done in other imaging modalities). This means that patient respiratory motion can substantially blur the acquired images. Compensation for motion can be performed by only processing the photons that are obtained at a specific motion state (e.g. exhalation) by referencing a motion signal over time. This motion signal can be extracted from the data (if sufficient photons can be detected for an accurate determination) or obtained from an external device that measures the patient's respiration (e.g. a respiratory belt). By employing motion compensation, the nuclear images become sharper and their quantitative accuracy increases. 

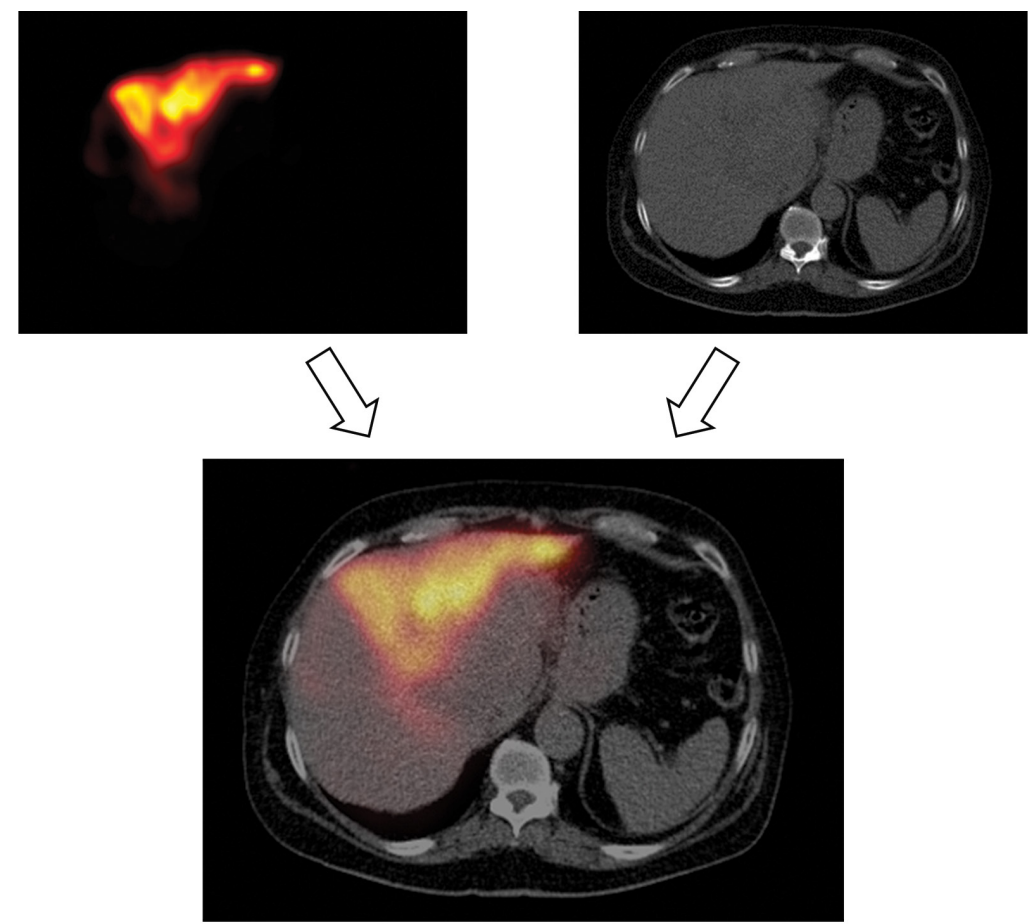

Figure 2: The SPECT reconstruction visualizes the radionuclide distribution (upper left). The CT reconstruction visualizes the anatomy (upper right). The combined SPECT/CT reconstruction complements the radionuclide distribution with anatomical details (lower).

\section{Image Reconstruction in SPECT}

Image reconstruction is notoriously challenging in SPECT because of two reasons. First, the activity that is injected in the patient is generally low to ensure that the patient receives little radiation dose. Consequently, the number of photons that are detected by the gamma camera is also low, which results in substantial noise in the acquired images. And second, many of the photons that are detected with the gamma camera have had physical interactions in the body of the patient, which changed their traveling direction (scatter) and their average signal amplitude (attenuation). These effects can substantially worsen the spatial resolution of the nuclear images (i.e. the images become less sharp) and introduce image artifacts.

To obtain the best image quality in nuclear imaging, there has been a major research focus on developing sophisticated mathematical algorithms and physical models that compensate for the above effects [16]. Some of the methods that are now routinely employed are attenuation correction by incorporating the anatomy of the patient in the SPECT reconstruction, scatter correction by measuring the energies of the detected photons to determine whether they had interactions (and discarding those that did), and resolution recovery by modeling the intrinsic spatial resolution of the gamma camera. 


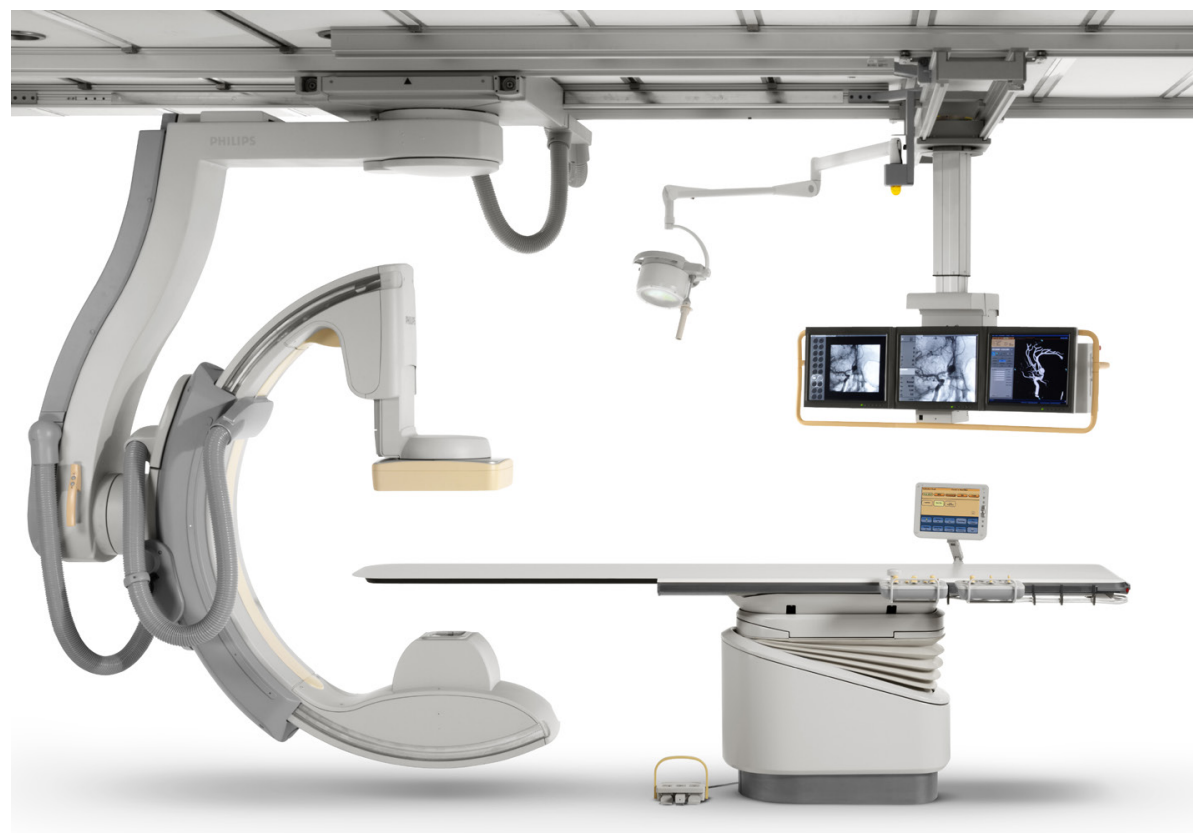

Figure 3: The c-arm CBCT scanner is frequently used for fluoroscopic image guidance of interventional procedures (displayed is the Allura Xper FD20; courtesy of Philips Healthcare). The scanner design is optimized for interventional imaging by its small size, flexibility, and capability of real-time imaging.

\section{Interventional Imaging}

Medical imaging is, in addition to disease diagnosis, increasingly used for guidance of (interventional) treatments. This dynamic setting places specific demands on the scanner designs, the acquisition protocols, and the subsequent data processing. In diagnostic imaging, the scanners can be large with a closed gantry (since no interaction with the patient is necessary), the acquisition can be relatively long, and the data processing can be performed in the hours following the acquisition. For interventional imaging, however, the scanners need to be flexible and compact to allow for smooth integration into the busy operation room and should preferably have an open gantry to enable easy access to the patient. Images are ideally obtained in real time so that direct feedback on the procedure is obtained. Furthermore, image acquisition should be performed as fast as possible to not prolong the interventional procedure and the data processing should be available within minutes.

\section{CBCT Scanner}

Several scanner designs have already been successfully employed in interventional imaging. One of these examples is the c-arm scanner design, which is a compact system that has much flexibility in scanner movement and sufficient space for the physicians to operate in. The c-arm design has been used to translate diagnostic CT imaging to an interventional setting. This was achieved by placing an $\mathrm{x}$-ray tube on one end of the c-arm and a flat panel detector on the opposite side (see Fig. 3). 
When the $\mathrm{x}$-ray tube is switched on, real-time planar fluoroscopic ( $\mathrm{x}$-ray) images are acquired, which can be used to visualize the position of a catheter and to map the vasculature. A visualization in $3 \mathrm{D}$ can be made by rotating the c-arm around the patient. This form of scanning is known as cone beam computed tomography (CBCT) [17]. The CBCT scanner acquires all projection angles in a matter of seconds and the scan reconstruction is generally available within a minute. This scanner system is hence an excellent example of how interventional imaging can be implemented.

\section{Flat Panel Detector}

The flat panel detector in the CBCT scanner is used for the detection of $\mathrm{x}$-rays [18]. Similar to the functioning of the gamma camera, the $\mathrm{x}$-ray photons interact with a scintillating material when entering the flat panel detector (often, cesium iodine) that converts the $\mathrm{x}$-ray photons into light. This light signal is digitized with photodiodes (instead of with photomultiplier tubes as in the gamma camera because of the greater signal strength). Flat panel detectors do not require a collimator for the detection process (as in the gamma camera) since the origin of the $\mathrm{x}$-rays is known (i.e. the $\mathrm{x}$-ray tube). However, to retrieve the best image quality, a grid that focuses on the $x$-ray tube is often placed before the flat panel detector so that scattered photons (which have a changed traveling direction) cannot pass the grid and are hence not detected by the flat panel detector. This grid is known as the anti-scatter grid [19].

\section{Interventional Nuclear Imaging}

For nuclear imaging (and especially hybrid imaging), it has proven much more difficult to translate the diagnostic scanner to an interventional setting. This is likely for two reasons. First, the SPECT and CT detectors are two separate entities on the gantry, which results in a relatively bulky device that is not flexible and has a closed gantry (Fig. 1). And second, the nuclear and x-ray images are acquired in sequence instead of simultaneously. Such sequential imaging results in motion artifacts in a dynamic setting and limits the interpretation of real-time acquired nuclear images. Nuclear imaging in the intervention room has hence so far hence only consisted of imaging with small (hand-held) gamma cameras that lack the ability to complement the nuclear images with real-time anatomical details $[20,21]$.

The lack of adequate nuclear imaging devices in the interventional setting illustrates that the translation from diagnostic to interventional imaging is not straightforward. Interventional nuclear imaging can likely only become a standard clinical option when a novel scanner is developed that meets all of the following criteria: the scanner should visualize nuclear images together with anatomical images in real time, the scanner should acquire images both in $2 \mathrm{D}$ (planar) and in $3 \mathrm{D}$ (reconstructions), the field of view of the scanner should be sufficient for imaging of large organs (e.g. the liver), the acquisition duration of a 3D scan should be short $(<10 \mathrm{~min})$, the data analysis should be completed quickly $(<1 \mathrm{~min}$ ), the nuclear images should be compensated for respiratory motion, the scanner should have an open gantry to provide sufficient space for the physicians, and the scanner should be compact and flexible. 

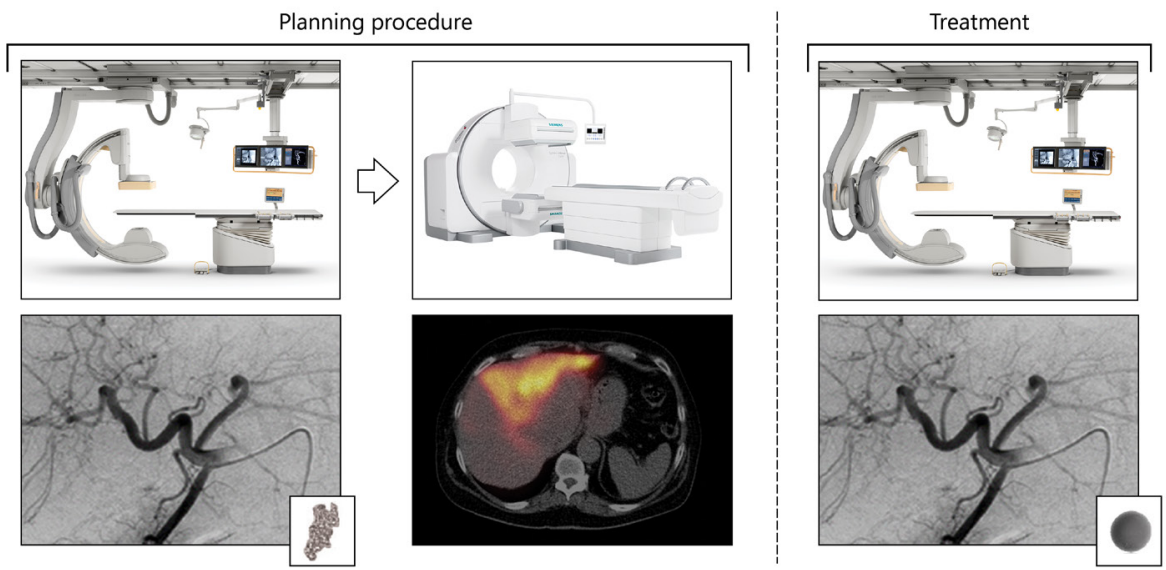

Figure 4: Imaging in the radioembolization workflow. The planning procedure starts by injecting MAA particles under fluoroscopic image guidance in the intervention room. The particle distribution is visualized by scanning the patient on a SPECT/CT scanner in the nuclear medicine department. When the patient proves to be a good candidate, the treatment is performed several weeks later by injecting the therapeutic microspheres. With an interventional nuclear scanner, it may become possible to merge the planning procedure with the treatment in a single-session procedure by allowing the visualization of the MAA particle distribution inside the intervention room.

\section{Applications}

Should interventional nuclear imaging become available in the clinic, it may be applied in several interventional procedures involving radionuclides, including sentinel node procedures [22], intraarterial injections of radiopharmaceuticals [23], and radio-guided surgeries [24]. One specific interventional procedure that has been pioneered in the University Medical Center Utrecht and is expected to benefit greatly from interventional nuclear imaging, is radioembolization for the treatment of liver cancer [25-27].

In radioembolization, radioactive microspheres (loaded with either yttrium-90 $\left({ }^{90} \mathrm{Y}\right)$ or holmium-166 $\left.\left({ }^{166} \mathrm{Ho}\right)\right)$ are injected into the tumor bloodstream with a microcatheter during a fluoroscopy-guided procedure. Due to the small size of the microspheres (25-32 $\mu \mathrm{m}$ in diameter), the particles primarily lodge in the small arteries where they deliver their therapeutic radiation. The radioembolization treatment is preceded by a planning procedure (performed up to weeks before treatment), in which the treatment is mimicked with macroaggregated albumin (MAA) particles that are labeled with technetium-99m $\left({ }^{99 \mathrm{~m}} \mathrm{Tc}\right)$ [28]. The planning procedure is used to determine whether a patient is a good candidate for treatment by determining safety measures such as the lung shunt fraction and potential other extrahepatic accumulations.

Interventional nuclear imaging may realize at least three potential benefits for radioembolization: i) the injection of activity can be performed under planar imaging guidance so that it is immediately known whether the correct tissues are targeted, ii) a SPECT/CT scan of the activity distribution can be acquired to quantitatively optimize the absorbed doses to the tissues [29,30], and iii) the planning procedure and the treatment may be merged in a single-session procedure to reduce the total time spent in the intervention room (see Fig. 4). 


\section{Outline}

This dissertation aims to translate diagnostic SPECT/CT imaging to an interventional setting. This requires the development of a scanner that meets the criteria for use in the intervention room and needs several methodological contributions to optimize the acquisition protocols and to shorten the acquisition and data processing durations. The contributions will be primarily evaluated for radioembolization as a potential application for interventional nuclear imaging.

\section{Interventional Scanner}

This dissertation starts by developing a SPECT scanner that can be used in the intervention room. A novel design is proposed that accomplishes a compact and mobile scanner with an open gantry (based on the c-arm construction of the CBCT scanner) that can furthermore provide simultaneous anatomical images to complement the nuclear images.

- CHAPTER 2 evaluates a prototype interventional SPECT scanner using digital simulations and phantom experiments.

- CHAPTER 3 evaluates a fully-developed interventional SPECT scanner using phantom experiments.

Instead of developing a new scanner, it may also be possible to adapt the CBCT scanner that is already available in the intervention room for fluoroscopic imaging. This approach could result in a more cost-effective method to achieve interventional nuclear imaging.

- CHAPTER 4 investigates the feasibility of nuclear imaging using the $\mathrm{x}$-ray flat panel detector of the CBCT scanner.

\section{Motion Compensation}

Patient respiratory motion is a major degrading factor for the quality of nuclear images. Conventional methods to compensate for respiratory motion require either external apparatus (e.g. a respiratory belt) or high activity levels. Both of these methods are undesired in an interventional setting and hence a new approach to motion compensation for interventional nuclear imaging should be developed.

- CHAPTER 5 introduces a novel method for motion compensation by extracting the motion signal from the anatomical images that are acquired simultaneously with the nuclear imaging in the interventional scanner and evaluates the method performance using digital simulations.

- CHAPTER 6 validates the proposed motion compensation technique using phantom experiments. 


\section{Fast Scanning}

Nuclear imaging scans conventionally need a long time ( $>20$ minutes) to acquire images with low noise. This scan duration should ideally be reduced to keep the interventional procedure as short as possible. One of the methods to reduce the scan duration is to improve the sensitivity of the system by making hardware modifications. One of the possible hardware modifications is to employ collimators that focus specifically on the organ of interest.

- CHAPTER 7 develops fast and quantitative reconstruction software for cone beam and multifocal collimators and evaluates the potential for scan duration shortening in liver imaging using simulations.

- CHAPTER 8 validates the performance of the developed reconstruction software for the multifocal collimator using phantom experiments.

A different method to accomplish scan duration shortening is to evaluate the radionuclide distribution characteristics during the acquisition so that the scan duration is personalized for every patient.

- CHAPTER 9 introduces a method for adaptive scan duration reduction by performing multiple fast detector rotations and terminating the scan when sufficient information on the distribution has been obtained.

\section{Fast Reconstruction}

Image reconstructions should be available quickly after scanning to ensure that the valuable time in the intervention room is not wasted. Current state-of-the-art reconstructors require several minutes to complete and hence should be accelerated.

- CHAPTER 10 develops a deep learning-based reconstruction method that generates images with a quality close to that of state-of-the-art reconstruction software in a matter of seconds.

The dissertation is summarized and discussed in CHAPTER 11. 


\section{References}

1. BushbergJT, Boone JM. The essential physics of medical imaging. Lippincott Williams \& Wilkins; 2011.

2. Cherry SR, Sorenson JA, Phelps ME. Physics in nuclear medicine. Elsevier Health Sciences; 2012.

3. Mariani G, Bruselli L, Kuwert T, Kim EE, Flotats A, Israel O, Dondi M, Watanabe N. A review on the clinical uses of SPECT/CT. European journal of nuclear medicine and molecular imaging. 2010;37(10):1959-85.

4. Boellaard R, Delgado-Bolton R, Oyen WJ, Giammarile F, Tatsch K, Eschner W, Verzijlbergen FJ, Barrington SF, Pike LC, Weber WA, Stroobants S. FDG PET/CT: EANM procedure guidelines for tumour imaging: version 2.0. European journal of nuclear medicine and molecular imaging. 2015;42(2):328-54.

5. Avram AM. Radioiodine scintigraphy with SPECT/CT: an important diagnostic tool for thyroid cancer staging and risk stratification. Journal of Nuclear Medicine. 2012;53(5):754-64.

6. Madsen MT. Recent advances in SPECT imaging. Journal of Nuclear Medicine. 2007;48(4):661-73.

7. Slomka PJ, Pan T, Germano G. Recent advances and future progress in PET instrumentation. Seminars in nuclear medicine 2016; 46(1):5-19.

8. Slomka PJ, Pan T, Berman DS, Germano G. Advances in SPECT and PET hardware. Progress in cardiovascular diseases. 2015;57(6):566-78.

9. Peterson TE, Furenlid LR. SPECT detectors: the Anger Camera and beyond. Physics in Medicine \& Biology. 2011;56(17):R145.

10. Anger HO. Scintillation Camera with Multichannel Collimators. Journal of Nuclear Medicine. 1964;5(7):515-31.

11. Van Audenhaege K, Van Holen R, Vandenberghe S, Vanhove C, Metzler SD, Moore SC. Review of SPECT collimator selection, optimization, and fabrication for clinical and preclinical imaging. Medical physics. 2015;42(8):4796-813.

12. Schillaci O. Hybrid SPECT/CT: a new era for SPECT imaging? European journal of nuclear medicine and molecular imaging. 2005;32(5):521-524

13. Shepp LA, Vardi Y. Maximum likelihood reconstruction for emission tomography. IEEE transactions on medical imaging. 1982;1(2):113-22.

14. Hudson HM, Larkin RS. Accelerated image reconstruction using ordered subsets of projection data. IEEE transactions on medical imaging. 1994;13(4):601-9.

15. Natterer F, Wübbeling F. Mathematical methods in image reconstruction. Siam; 2001.

16. Bailey DL, Willowson KP. Quantitative SPECT/CT: SPECT joins PET as a quantitative imaging modality. European journal of nuclear medicine and molecular imaging. 2014;41(1):17-25.

17. Floridi C, Radaelli A, Abi-Jaoudeh N, Grass M, De Lin M, Chiaradia M, Geschwind JF, Kobeiter H, Squillaci E, Maleux G, Giovagnoni A. C-arm cone-beam computed tomography in interventional oncology: technical aspects and clinical applications. La radiologia medica. 2014;119(7):521-32.

18. Rowlands JA, Yorkston J. Flat panel detectors for digital radiography. Handbook of medical imaging. 2000;1:223-328.

19. Veldkamp WJ, Thijssen MA, Karssemeijer N. The value of scatter removal by a grid in full field digital mammography. Medical Physics. 2003:1712-8.

20. Beijst C, Kunnen B, Lam MG, de Jong HW. Technical advances in image guidance of radionuclide therapy. Journal of nuclear medicine technology. 2017;45(4):272-9. 
21. Tsuchimochi M, Hayama K. Intraoperative gamma cameras for radioguided surgery: technical characteristics, performance parameters, and clinical applications. Physica Medica. 2013;29(2):126-38.

22. Vidal-Sicart S, Paredes P, Zanón G, Pahisa J, Martinez-Román S, Caparrós X, Vilalta A, Rull R, Pons F. Added value of intraoperative real-time imaging in searches for difficult-to-locate sentinel nodes. Journal of Nuclear Medicine. 2010;51(8):1219-25.

23. Ebbers SC, Barentsz MW, Braat AJ, Lam MG. Intra-Arterial Peptide Receptor Radionuclide Therapy for Neuroendocrine Tumor Liver Metastases. Digestive Disease Interventions. 2019;3(01):081-90.

24. Povoski SP, Neff RL, Mojzisik CM, O’Malley DM, Hinkle GH, Hall NC, Murrey DA, Knopp MV, Martin EW. A comprehensive overview of radioguided surgery using gamma detection probe technology. World journal of surgical oncology. 2009;7(1):11.

25. Kennedy A, Coldwell D, Sangro B, Wasan H, Salem R. Radioembolization for the treatment of liver tumors: general principles. American journal of clinical oncology. 2012;35(1):91-9.

26. van Roekel C, Braat AJ, Smits ML, Bruijnen RC, de Keizer B, Lam MG. Radioembolization. Clinical Nuclear Medicine, 2020;961-999.

27. Bastiaannet R, Kappadath SC, Kunnen B, Braat AJ, Lam MG, de Jong HW. The physics of radioembolization. EJNMMI physics. 2018;5(1):22.

28. Garin E, Rolland Y, Laffont S, Edeline J. Clinical impact of $99 \mathrm{~m}$ Tc-MAA SPECT/CT-based dosimetry in the radioembolization of liver malignancies with 90 Y-loaded microspheres. European journal of nuclear medicine and molecular imaging. 2016;43(3):559-75.

29. Braat AJ, Kappadath SC, Bruijnen RC, van den Hoven AF, Mahvash A, de Jong HW, Lam MG. Adequate SIRT activity dose is as important as adequate chemotherapy dose. The Lancet Oncology. 2017;18(11):e636.

30. Smits ML, Elschot M, Sze DY, Kao YH, Nijsen JF, Iagaru AH, de Jong HW, van den Bosch MA, Lam MG. Radioembolization dosimetry: the road ahead. Cardiovascular and interventional radiology. 2015;38(2):261-9. 


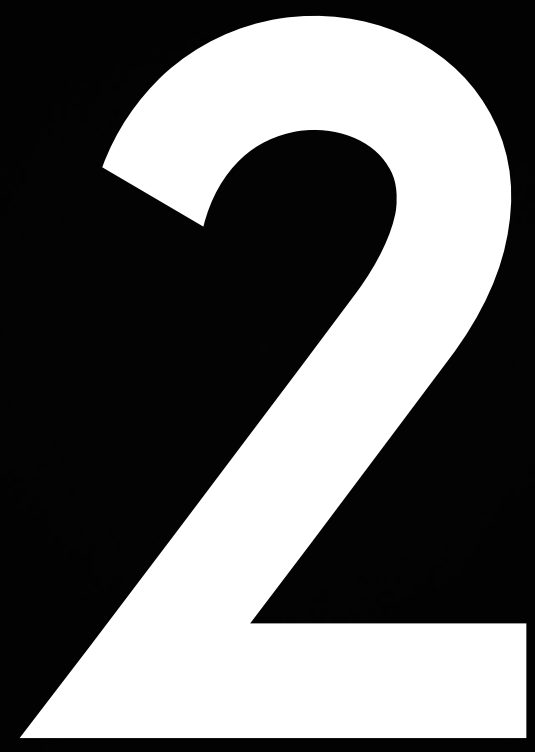




\section{A PROTOTYPE INTERVENTIONAL SCANNER}

PUBLISHED AS:

MMA Dietze, B Kunnen, S van der Velden, JHL Steenbergen, WJC Koppert, MA Viergever, HWAM de Jong Performance of a Dual-Layer Scanner for Hybrid SPECT/CBCT 64(10):105020 (2019) 


\begin{abstract}
Purpose

Fluoroscopic procedures involving radionuclides would benefit from interventional nuclear imaging by obtaining real-time feedback on the activity distribution. We have previously proposed a dual-layer detector that offers such procedural guidance by simultaneous fluoroscopic and nuclear planar imaging. Acquisition of single photon computed tomography (SPECT) and cone beam computed tomography (CBCT) could provide additional information on the activity distribution. This study investigates the feasibility and the image quality of simultaneous SPECT/CBCT, by means of phantom experiments and simulations.
\end{abstract}

\title{
Methods
}

Simulations were performed to study the obtained reconstruction quality for (i) clinical SPECT/ CT, (ii) a dual-layer scanner configured with optimized hardware, and (iii) our (non-optimized) dual-layer prototype. Experiments on an image quality phantom and an anthropomorphic phantom (including extrahepatic depositions with volumes and activities close to the median values encountered in hepatic radioembolization) were performed with a clinical SPECT/CT scanner and with our dual-layer prototype. Nuclear images were visually and quantitatively evaluated by measuring the tumor/non-tumor $(\mathrm{T} / \mathrm{N})$ ratio and contrast-to-noise ratio $(\mathrm{CNR})$.

\section{Results}

The simulations showed that the maximum obtained CNR was $38.8 \pm 0.8$ for the clinical scanner, $30.2 \pm 0.9$ for the optimized dual-layer scanner, and $20.8 \pm 0.4$ for the prototype scanner. T/N ratio showed a similar decline. The phantom experiments showed that performing simultaneous SPECT/CBCT is feasible. The CNR obtained from the SPECT reconstruction of largest sphere in the image quality phantom was 43.1 for the clinical scanner and 28.6 for the developed prototype scanner. The anthropomorphic phantom showed that the extrahepatic depositions were detected with both scanners.

\section{Conclusion}

A dual-layer detector is able to simultaneously acquire SPECT and CBCT. Both CNR and T/N ratio are worse than that of a clinical system, but the phantom experiments showed that extrahepatic depositions with volumes and activities close to the median values encountered in hepatic radioembolization could be distinguished. 


\section{Introduction}

In recent years, significant progress has been made in the development of hybrid medical scanners. For instance, single photon emission computed tomography (SPECT) scans are now routinely corrected for photon attenuation by using the included computed tomography (CT) component of SPECT/CT devices [1]. Together with software improvements in e.g. scatter correction and resolution modeling, SPECT/CT is increasingly considered a quantitative modality [2]. These improvements have initiated new opportunities in the evaluation of administered activity [3].

For example, in hepatic radioembolization, where yttrium-90 $\left({ }^{90} \mathrm{Y}\right)$ labeled microspheres are injected into the liver $[4,5]$, a separate pre-treatment fluoroscopy-guided interventional procedure with technetium-99m-labeled macroaggregated albumin ( ${ }^{99 m}$ Tc-MAA) is performed. After this procedure, the lung shunt fraction and potential extrahepatic depositions are determined on SPECT/CT to decide whether the treatment can safely continue. Ideally, the results from this SPECT/CT scan would be directly available during the interventional procedure, so that both ${ }^{99 \mathrm{~m}} \mathrm{Tc}$ pre-treatment and ${ }^{90} \mathrm{Y}$ treatment administrations could be merged into a single fluoroscopic interventional session [6].

However, the activity distribution can currently only be visualized using the scanners at the nuclear medicine department. Evaluation during the fluoroscopic interventional session would thus require patients to be transferred across the hospital, which takes a substantial amount of time. Additionally, the catheter used to inject activity could shift in position, creating an unintended difference in the vascular flow (and thus in the distribution) between several administrations [7]. The combination of these effects makes transportation unattractive for clinical practice; a dedicated scanner in the intervention room would be preferred.

With this application in mind, a compact hybrid scanner [8] was previously proposed. The duallayer detector, consisting of a cone beam CT (CBCT) flat panel positioned in front of a gamma camera, can acquire nuclear and fluoroscopic projections with the same field of view (FOV) simultaneously (see Fig. 1 for an example). The use of a C-arm gantry resulted in a compact scanner that would enable seamless integration in the intervention room. For hepatic radioembolization, this means that both anatomy and activity distribution could be simultaneously visualized. Such information is currently not available and could aid radionuclide administration by offering direct feedback to the physician.

The proposed dual-layer scanner has been evaluated for a $2 \mathrm{D}$ setting (i.e., planar imaging). Additional information on the activity distribution can be achieved if a complete rotation around the patient is performed; resulting in a hybrid SPECT/CBCT. Quantitative results can then be retrieved by including attenuation and scatter correction in the SPECT reconstruction. The dual-layer scanner has potential advantages over a clinical SPECT/CT system by achieving intrinsically registered reconstructions (as the detectors capture the same FOV at the same time) and compensation for respiratory motion in the SPECT reconstruction (exploiting the simultaneous nature of the acquired projections). 


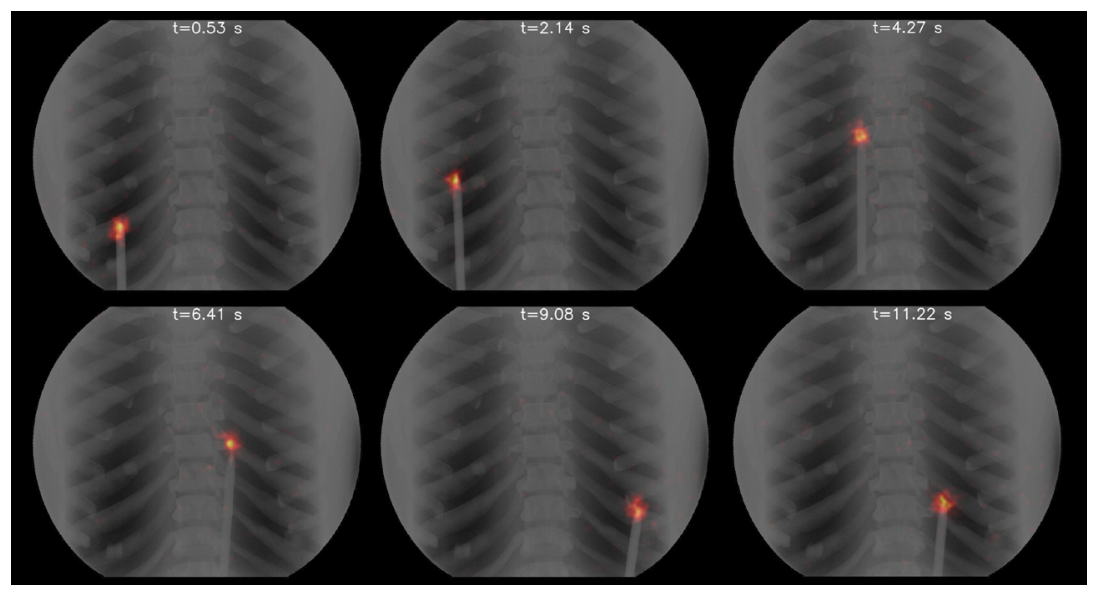

Figure 1: An illustration of how nuclear (color) and fluoroscopic (gray-scale) information is captured simultaneously in planar imaging. A cobalt-57 pen marker was moved inside the thorax of an anthropomorphic phantom. Details of the acquisition can be found elsewhere [8].

Since there exist substantial differences in system design between the dual-layer scanner and a clinical SPECT/CT scanner, the SPECT reconstruction quality of the dual-layer scanner will be lower. The extent of this is, however, currently not clear. For our proposed specific application in hepatic radioembolization, image quality requirements could be relaxed as compared with a diagnostic SPECT/CT scan. Accordingly, the lower quality of the dual-layer scanner would not necessarily impede clinical usage in interventional procedures.

This study evaluates the image quality and quantitative accuracy of the dual-layer scanner in comparison to a clinical SPECT/CT scanner via simulations and phantom experiments. For the simulations, the dual-layer design will be evaluated for our developed (non-optimized) prototype and for a scanner optimized for gamma transmission, to illustrate the potential future capabilities of the dual-layer design.

\section{Methods}

\section{Studied scanners}

This study compared the performance of three scanners, whose configurations are shown in Tab. 1.

\section{Clinical SPECT/CT}

The clinical SPECT/CT scanner was a Symbia T16 (Siemens Healthineers, Erlangen, Germany). This scanner has two detector heads, was mounted with a low-energy high-resolution (LEHR) parallel hole collimator $(24.05 \mathrm{~mm}$ hole length, $1.11 \mathrm{~mm}$ hole diameter and $0.16 \mathrm{~mm}$ septal thickness) and is able to perform a body-tracing orbit. The effective FOV of the gamma camera was $53.3 \times 38.7 \mathrm{~cm}^{2}$. The CT scan is always performed separately from the SPECT scan. Intrinsic spatial resolution was $3.8 \mathrm{~mm}$ full width at half maximum (FWHM) and the energy resolution was $9.9 \%$ [9]. 
Table 1: Configurations of the clinical scanner, the optimized dual-layer scanner, and the prototype dual-layer scanner, evaluated in this study.

\begin{tabular}{l|ccc} 
& Clinical & Optimized & Prototype \\
\hline Mobile & No & Yes & No \\
Detector heads & Two & One & One \\
CT acquisition & Separate & Simultaneous & Simultaneous \\
Detector orbit & Body-tracing & Parameterized & Circular \\
Flat panel transmission & - & $65 \%$ & $52 \%$ \\
Flat panel thickness & - & $2.0 \mathrm{~cm}$ & $7.5 \mathrm{~cm}$ \\
Collimator & Parallel hole $(\mathrm{LEHR})$ & Cone beam $(\mathrm{LEHR})$ & Cone beam $($ Custom $)$ \\
Gamma camera FOV & $53.3 \times 38.7 \mathrm{~cm}^{2}$ & $60.0 \times 45.0 \mathrm{~cm}^{2}$ & $40.0 \times 24.0 \mathrm{~cm}^{2}$
\end{tabular}

\section{Optimized Dual-Layer}

The proposed dual-layer scanner is shown in Fig. 2. A flat panel detector is positioned in front of a gamma camera with cone beam LEHR collimator with $100 \mathrm{~cm}$ focal distance. The $\mathrm{x}$-ray tube is positioned at $100 \mathrm{~cm}$ from the collimator, so that nuclear and fluoroscopic projections capture the same FOV. The majority of $\mathrm{x}$-rays are absorbed in the flat panel, whilst the gamma photons are, due to their relatively higher energy, more likely to pass through the flat panel and be detected by the gamma camera. This hybrid scanner can, due to its compact configuration, be mounted on a custom C-arm.

Only one detector head will be present on the dual-layer scanner, which increases the noise levels in comparison with the clinical SPECT/CT scanner with two detector heads. The introduction of the flat panel in front of the gamma camera also decreases the number of detected gamma photons. The expected gamma transmission for an optimized flat panel $(2 \mathrm{~cm}$ in thickness $)$ at $140 \mathrm{keV}$ is between 65 and $73 \%$ [8]; transmission of $65 \%$ was chosen for this study. The effective FOV of the gamma camera would need to be somewhat larger than a clinical scanner, to account for the cone geometry; a FOV of $60.0 \times 45.0 \mathrm{~cm}^{2}$ is likely sufficient. The gamma camera was assumed to have the performance as in the prototype dual-layer scanner: intrinsic spatial resolution of $3.1 \mathrm{~mm}$ FWHM and energy resolution of $9.3 \%$.

No collision monitors will be present on the scanner, in order to keep the design simple. This means that a regular body-tracing orbit will not be performed. Rather, by measuring the detector-patient distance at a few points, a parameterized orbit can be constructed (see Fig. 3 for an illustration). Such an orbit is not optimal, since it needs to account for many possible patient shapes and sizes, but will be substantially better in tracking the patient contour than a circular orbit.

\section{Prototype Dual-Layer}

The developed prototype scanner, see Fig. 4, was configured with the same dual-layer approach as in Fig. 2, but was not yet mounted on a C-arm. The phantom was therefore positioned on a rotating table, which means that a circular orbit was performed. The gamma camera and the flat 


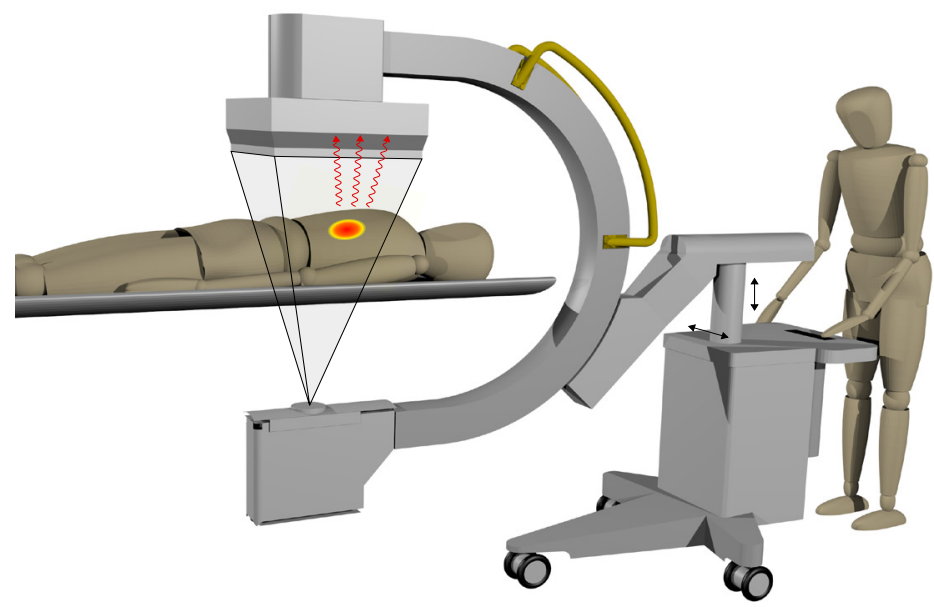

Figure 2: An illustration of the optimized dual-layer scanner. The flat panel is placed in front of the cone beam collimator and the gamma camera. The entire C-arm is translated in order to follow the patient contour.

panel were positioned as close to each other as possible. The focal spot of the $\mathrm{x}$-ray tube (Veradius; Philips Healthcare, Best, The Netherlands) was positioned at $98 \mathrm{~cm}$ from the front of the cone beam collimator and was aligned with the center of the detectors.

The used flat panel was adjusted from a commercially available product (Pixium 3040; Trixell, Moirans, France) by reducing the thickness of the back shielding plate, which resulted in a gamma transmission rate of $52 \%$ at $140 \mathrm{keV}$ [8]. The flat panel thickness was $7.5 \mathrm{~cm}$ and the effective FOV was $38.2 \times 29.6 \mathrm{~cm}^{2}$. The difference with an optimized flat panel (as above) comes from the printed circuit board electronics that would ideally be placed outside of the detector by extending the flat cables, which would allow for a more compact detector housing.

No adjustments were made to the gamma camera (P3000; Inter Medical Medizinservice, Lübbecke, Germany), which had an effective FOV of $40.0 \times 24.0 \mathrm{~cm}^{2}$, intrinsic spatial resolution of $3.1 \mathrm{~mm}$ FWHM, and energy resolution of $9.3 \%$. The cone beam collimator (Nuclear Fields, VortumMullem, The Netherlands) had a focal distance of $98 \mathrm{~cm}, 40.00 \mathrm{~mm}$ hole length, $1.90 \mathrm{~mm}$ hole diameter and $0.25 \mathrm{~mm}$ septal thickness. These hole dimensions were slightly larger than those of a regular LEHR collimator (but had approximately the same aspect ratio), owing to limitations of the manufacturing process.

\section{Simulation study}

There are substantial differences in hardware between the clinical SPECT/CT scanner and the dual-layer scanners. The effects of the differences on the final nuclear reconstruction quality were evaluated in a simulation study. The simulation study functions as a reference for observed performance in the phantom experiments, finds the influence of individual components, evaluates the expected performance of the optimized design, and allows a more rigorous evaluation than with the phantom experiments. 

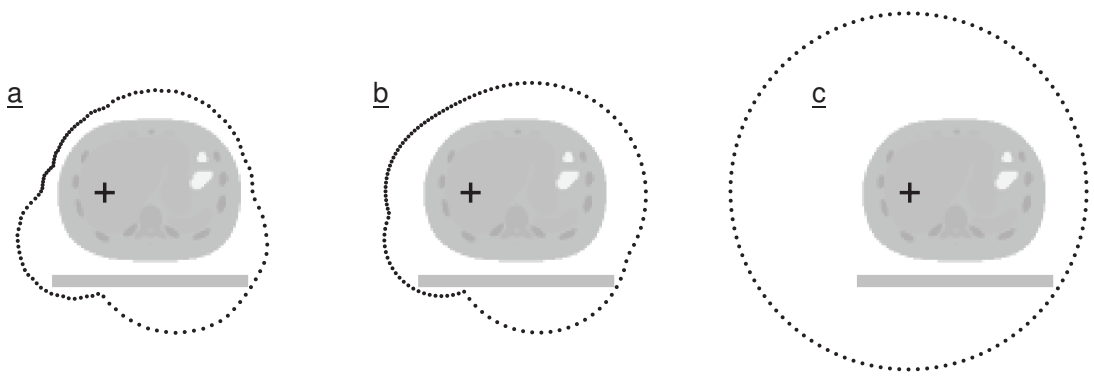

Figure 3: An illustration of the detector position for the several orbit options with the XCAT phantom: (a) body-tracing, (b) parameterized, and (c) circular. The detector is centered on the center of mass of the liver, visualized by the marker. Under the patient bed, the detector increases its distance to the rotation point to avoid collision with the patient bed (for the body-tracing and parameterized orbit). The orbits are asymmetric, because the rotation point lies in the center of mass of the liver.

The XCAT phantom [10] was used for the creation of realistic digital patient data, using the standard settings of a $95 \mathrm{~kg}$ male. The phantom contained a total of $150 \mathrm{MBq}{ }^{99 \mathrm{~m}} \mathrm{Tc}$, in which a tumor with respectively 20,30 or $50 \mathrm{~mm}$ radius was added at an uptake ratio of 5:1. Activity in the lungs was added at a lung-shunting fraction (LSF) of 5.00\%. A flat patient bed (as in the intervention room) was positioned $5 \mathrm{~cm}$ underneath the phantom. The activity and attenuation maps were configured on a $240 \times 240$ x 300 matrix, with $4.7 \mathrm{~mm}$ isotropic voxels. Attenuation maps were assumed to be known perfectly from the CT or CBCT scan, hence no effects from these maps on SPECT reconstruction were included.

The Utrecht Monte Carlo System (UMCS) [11,12] was used to generate projections. UMCS simulates the photon physics and detector response in a fast manner, making it possible to acquire noise-free projections. The required collimator point spread functions were generated in GATE [13] for every detector configuration. The effects of measurement time and nuclear activity were incorporated by addition of Poisson noise to the projections. The gamma camera was centered on the liver center-of-mass.

Simulated scan time was 10 minutes, as is what we envision for interventional procedures. Projections were simulated with 120 angles and photons were captured in an energy window of $15 \%$ around the $140 \mathrm{keV}$ photopeak. Reconstructions were performed with 10 iterations and 8 subsets and a postreconstruction Gaussian filter of $2.5 \mathrm{~mm}$ FWHM was applied. The effects of potential truncation in SPECT reconstructions owing to the different gamma camera sizes were not taken into account, as it was previously shown that truncation does not influence the accuracy of imaging in the region seen by all angles [12]. Ten noise realizations were performed per setting to study the stability of the various measures.

Reconstruction quality was compared on tumor to non-tumor $(\mathrm{T} / \mathrm{N})$ ratio (a crucial measure for dosimetry) and contrast-to-noise ratio (CNR) (as a measure of the visual quality). The T/N ratio was measured at iteration 10 . The reported CNR was the maximum retrieved value over all 


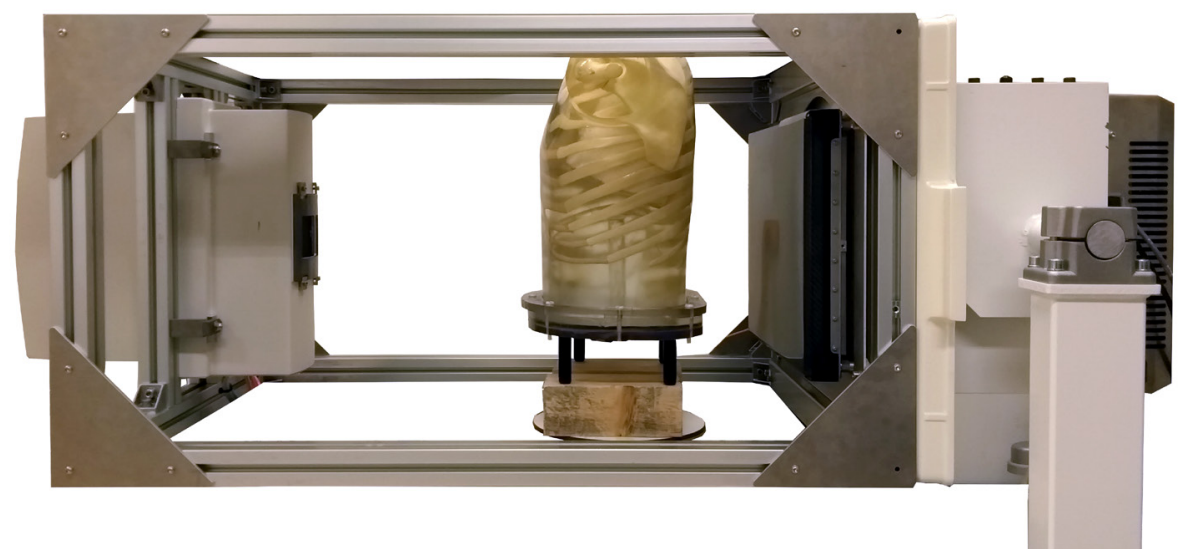

Figure 4: The prototype dual-layer scanner. Whilst the detector is not yet mounted on a C-arm, the same duallayer approach as in Fig. 2 can be observed. The phantom gives an illustration of where a patient would be located.

iterations, inasmuch as CNR is a trade-off between recovered activity and background noise and will differ as a function of reconstruction convergence rate. Tumor and background liver masks were available from the initial phantom creation. The background liver mask was eroded by 2 voxels to reduce partial volume effects.

The clinical scanner was taken as the initial configuration for the simulations. The impact of changing an individual scanner component was hence evaluated for the decrease of detector heads from two to one, for the change of detector orbits from body tracing to parameterized or circular, for the introduction of a flat panel in front of the gamma camera, and for changing the collimator from parallel to cone beam. Furthermore, the three scanners, with all individual components combined together (e.g. for the prototype dual-layer scanner: one head, circular orbit, prototype flat panel, and prototype cone beam collimator), were also simulated.

\section{Phantom study}

A phantom study was performed to illustrate the image quality retrieved by the prototype dual-layer scanner and to evaluate the impact of simultaneous imaging. A custom image quality phantom and a realistic anthropomorphic phantom were scanned on both the clinical scanner and the developed prototype scanner. Images of the used phantoms are shown in Fig. 5.

The image quality phantom consisted of the spheres from a regular image quality (IQ) phantom $(37,28,22,17,13$, and $10 \mathrm{~mm}$ diameter) (IEC NEMA 2007) in their usual pattern, but they were enclosed in a different cylinder (Hoffman Brain phantom). The latter was used because it is somewhat smaller than the regular IQ enclosing, which ensured that no truncation effects affected the acquisition. The spheres were filled with an uptake ratio of 10:1 compared with the background compartment. The spheres and background were filled with water and a total of $150 \mathrm{MBq}^{99 \mathrm{~m}} \mathrm{Tc}$ was used. 

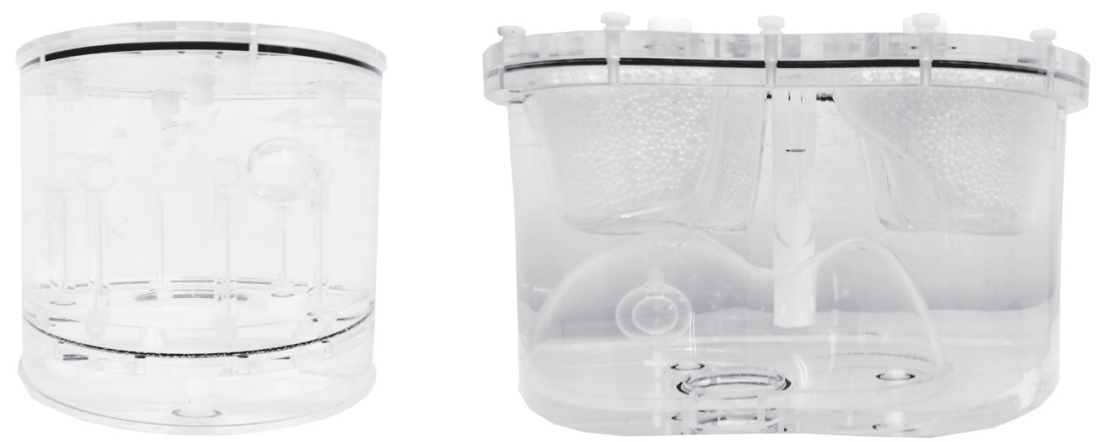

Figure 5: The used phantoms in this study. On the left, the image quality phantom, which was used to study the resolution and noise of the scanners. On the right, the anthropomorphic phantom, which is clinically relevant.

The anthropomorphic phantom was modified from a commercially available phantom (Anthropomorphic Torso Phantom: ECT/TOR/P) by inclusion of a sphere with cold core (18.6 $\mathrm{ml}$ active volume, 5.6:1 uptake ratio relative to the background liver insert of $1200 \mathrm{ml}$ ) inside the liver and three spheres (4.1,7.9, and $15.7 \mathrm{ml}$, all with 2.8:1 uptake ratio) in the torso. The lungs were filled with $5.4 \%$ of the total activity. The phantom was filled with water and a total of $160 \mathrm{MBq}$ ${ }^{99 \mathrm{~m}} \mathrm{Tc}$ was present. The phantom was configured in this way to represent the median volume $(6.8 \mathrm{ml})$ and activity (1.3\% of the total injected activity) of extrahepatic depositions encountered in hepatic radioembolization [14].

In the prototype scanner, the rotation was continuous and the CBCT scan was performed simultaneously with SPECT at an acquisition rate of $3.75 \mathrm{~Hz}$, hence $2250 \mathrm{x}$-ray projections were taken. The list mode SPECT data was binned into 120 projections. The image quality phantom was scanned using $50 \mathrm{kVp}, 4 \mathrm{mAs}$, and $13.5 \mathrm{~cm}$ rotation radius. The anthropomorphic phantom was scanned using $60 \mathrm{kVp}, 12 \mathrm{mAs}$, and $20.5 \mathrm{~cm}$ rotation radius. Reconstructions of the fluoroscopic projections were performed with the GPU-based iterative CBCT reconstruction TIGRE toolbox [15], using the SIRT algorithm with 50 iterations. The resulting CBCT reconstruction was used as attenuation map by applying a previously made look-up table.

For the clinical scanner, the SPECT (in step-and-shoot mode) and CT data were acquired sequentially. Again, 120 SPECT projections were acquired. The clinical scanner used $110 \mathrm{kVp}$ with $14 \mathrm{mAs}$ or $68 \mathrm{mAs}$ for the image quality and anthropomorphic phantom, respectively. The attenuation maps were retrieved by means of the clinical software. Nuclear scanning time was 10 minutes for both scanners.

Since the anthropomorphic phantom did not fit completely into the prototype FOV, it could only be partially reconstructed. The resulting CBCT reconstruction was hence registered to the attenuation map of the clinical scanner by performing a rigid transformation with Elastix [16]. The clinical attenuation map could in this way be used for SPECT reconstruction of the prototype. Such a registration would also be required in a clinical interventional setting if the same size flat panel is used. 

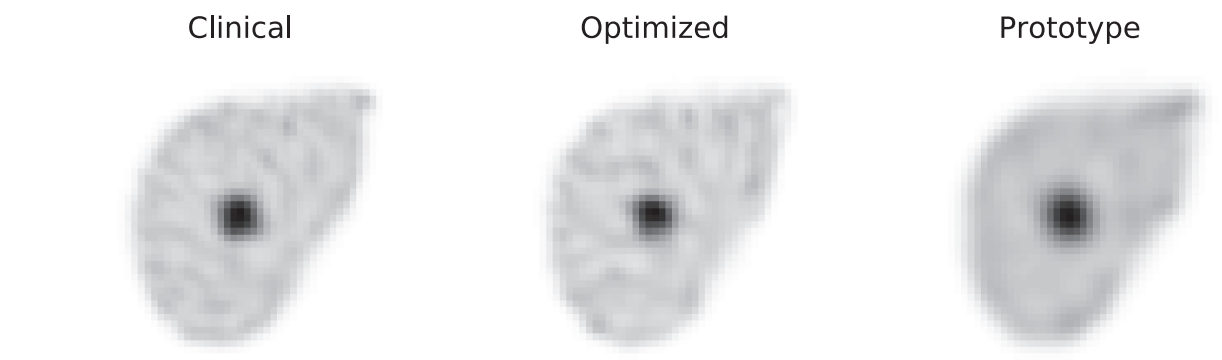

Figure 6: Reconstruction examples for the scanners at iteration 10 for the sphere of $30 \mathrm{~mm}$ radius.

Nuclear reconstructions for both the prototype and the clinical scanner were performed using UMCS. A photopeak window of $15 \%$ around $140 \mathrm{keV}$ was used and 10 iterations with 8 subsets were performed. For the prototype scanner, the pulses of the x-ray tube were cut from the SPECT list mode data by applying temporal selection to ensure only gamma photons were used. The anthropomorphic phantom was reconstructed on a $128 \times 128 \times 128$ grid (voxel size of $4.79 \mathrm{~mm}$ ), because this setting is currently used in clinical practice in our institute. The IQ phantom was reconstructed on a $256 \times 256 \times 256$ grid (isotropic voxel size of $2.40 \mathrm{~mm}$ ), because this smaller voxel size allowed a more detailed visualization. A post-reconstruction Gaussian filter of $2.5 \mathrm{~mm}$ FWHM was applied to both SPECT reconstructions.

For the image quality phantom, $\mathrm{T} / \mathrm{N}$ ratio and $\mathrm{CNR}$ were calculated. Lesion masks were made by thresholding the SPECT reconstruction to determine the spheres' centers of masses and then growing the volumes to the reported sphere sizes. The background volume was created by thresholding the active volume, removing the sphere masks and eroding the resulting mask by 3 voxels. For the anthropomorphic phantom, the reconstructions were solely visually assessed.

\section{Results}

\section{Simulation study}

Examples of reconstructions from the simulation study are shown for illustration purposes in Fig. 6 for the clinical, optimized, and prototype scanners. The clinical and optimized scanners generated the sharpest reconstructions, whereas the reconstruction was blurrier for the prototype scanner.

The effect on reconstruction quality of the individual scanner components and the components combined into the scanner configurations are collected in Tab. 2. The maximum CNR and LSF are reported solely for the $30 \mathrm{~mm}$ tumor, since these measures were less dependent on sphere size than $\mathrm{T} / \mathrm{N}$ ratio.

The change in going from two detector heads to one resulted in higher noise levels in the reconstruction, since the time per view was halved. Therefore, CNR decreased for the dual-layer scanners. This reduction could be corrected for if the total scan time would be doubled. $\mathrm{T} / \mathrm{N}$ ratio was largely unaffected by the number of detector heads. 
Table 2: Overview of the results from the simulation study by changing a specific component with regards to the clinical scanner and by combining all individual components.

\begin{tabular}{l|ccc|c|c} 
& \multicolumn{3}{|c|}{ T/N ratio } & Max CNR & LSF [\%] \\
& $20 \mathrm{~mm}$ & $30 \mathrm{~mm}$ & $50 \mathrm{~mm}$ & & \\
\hline Clinical scanner & $3.88 \pm 0.16$ & $4.28 \pm 0.05$ & $4.64 \pm 0.03$ & $37.6 \pm 1.5$ & $5.10 \pm 0.03$ \\
& & & & & \\
Single detector head & $3.95 \pm 0.13$ & $4.27 \pm 0.08$ & $4.66 \pm 0.03$ & $30.1 \pm 0.9$ & $5.04 \pm 0.05$ \\
& & & & & \\
Parameterized orbit & $3.80 \pm 0.12$ & $4.24 \pm 0.06$ & $4.64 \pm 0.04$ & $36.6 \pm 1.2$ & $5.12 \pm 0.03$ \\
Circular orbit & $2.81 \pm 0.12$ & $3.95 \pm 0.07$ & $4.40 \pm 0.03$ & $28.8 \pm 0.7$ & $5.25 \pm 0.03$ \\
& & & & & \\
Optimized flatpanel & $3.71 \pm 0.17$ & $4.20 \pm 0.05$ & $4.60 \pm 0.03$ & $33.2 \pm 1.3$ & $5.10 \pm 0.02$ \\
Prototype flat panel & $3.53 \pm 0.19$ & $4.06 \pm 0.05$ & $4.51 \pm 0.03$ & $28.4 \pm 1.1$ & $5.11 \pm 0.07$ \\
& & & & & \\
LEHR cone beam collimator & $3.87 \pm 0.11$ & $4.27 \pm 0.04$ & $4.64 \pm 0.04$ & $42.2 \pm 1.2$ & $5.16 \pm 0.03$ \\
Custom cone beam collimator & $3.81 \pm 0.12$ & $4.28 \pm 0.05$ & $4.64 \pm 0.02$ & $43.2 \pm 1.3$ & $5.18 \pm 0.03$ \\
& & & & & \\
Optimized scanner & $3.74 \pm 0.11$ & $4.19 \pm 0.06$ & $4.59 \pm 0.04$ & $31.2 \pm 1.2$ & $5.16 \pm 0.04$ \\
Prototype scanner & $2.38 \pm 0.07$ & $3.68 \pm 0.10$ & $4.24 \pm 0.03$ & $22.0 \pm 1.0$ & $5.39 \pm 0.05$
\end{tabular}

Body tracing allowed the detector to get closest to the patient body, the parameterized orbit needed somewhat more space to account for all possible patients, and the circular orbit had a radius of 34 $\mathrm{cm}$. When the gamma camera was positioned further away from the patient, the system resolution decreased. The $\mathrm{T} / \mathrm{N}$ ratio and the $\mathrm{CNR}$, therefore, both became worse in the dual-layer designs.

The effects on the reconstruction of introducing a flat panel were twofold. First, although the resolution recovery was incorporated in the reconstruction, the system resolution still decreased, because the thickness of the flat panel induced a larger phantom distance. Second, the number of detected nuclear counts reduced as a consequence of the attenuation of gamma photons. Therefore, both the $\mathrm{T} / \mathrm{N}$ ratio and the $\mathrm{CNR}$ decreased when introducing a flat panel in front of the gamma camera.

The introduction of cone beam collimators improved noise levels somewhat compared with the parallel hole collimator, thanks to the increased system sensitivity. The CNR was thus increased. However, this improvement in reconstruction quality was negligible as compared with the effects of the other studied detector components. $\mathrm{T} / \mathrm{N}$ ratio was largely unaffected, inasmuch as the system resolution remained the same.

Finally, all individual changes were combined to assess their joint influence on the image quality of the several studied scanners. Evidently, the clinical scanner had the best performance. The prototype possessed lower $\mathrm{T} / \mathrm{N}$ ratios and lower $\mathrm{CNR}$. The optimized dual-layer scanner had a lower CNR, but $\mathrm{T} / \mathrm{N}$ ratios approached that of the clinical scanner. 

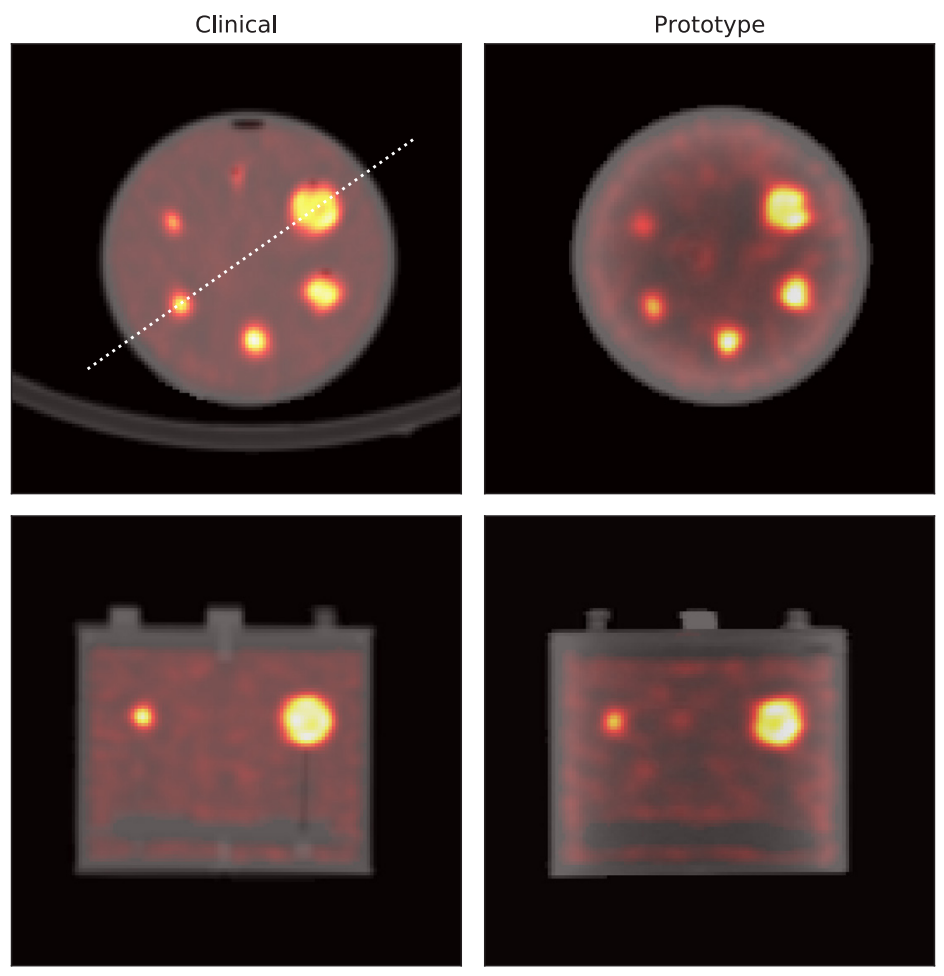

Figure 7: The reconstructions of the image quality phantom at iteration 10 for the clinical SPECT/CT (left) and the prototype SPECT/CBCT (right) of a transverse (top) and coronal (bottom) slice. The white dotted line denotes the position of the coronal slice, and of the profile shown in Fig. 8a.

\section{Phantom study}

The reconstructions of the image quality phantom for the clinical and prototype scanners are shown in Fig. 7. The CBCT reconstructions made with the prototype scanner had no patient bed present, but the phantom showed the same shape and values as in the clinical scanner and was thus used for attenuation correction. The maximum CNR of the largest sphere in the reconstruction was 43.1 for the clinical scanner and 28.6 for the prototype scanner, which is in line with the simulations.

The profile over the spheres of 22 and $17 \mathrm{~mm}$ diameter is shown in Fig. 8a. The profile was normalized to the total activity in the background of both SPECT reconstructions. The peak of the small sphere in the prototype scanner reached a lower maximum than the clinical scanner. This behavior is confirmed by the $\mathrm{T} / \mathrm{N}$ ratios for all spheres in Fig. $8 \mathrm{~b}$, which shows that the relative difference between the scanners increased toward smaller spheres. These results were in agreement with the trends on the $\mathrm{T} / \mathrm{N}$ ratio observed in the simulation study.

The reconstructions of the anthropomorphic phantom are shown in Fig. 9. Because the phantom was larger than the FOV of the prototype scanner, it could only be partially reconstructed. Although the bone insert in the prototype scanner did not stand out as much as in the clinical scanner, the lungs were clearly visible. Hence, it was possible to register the CBCT from the prototype scanner to 

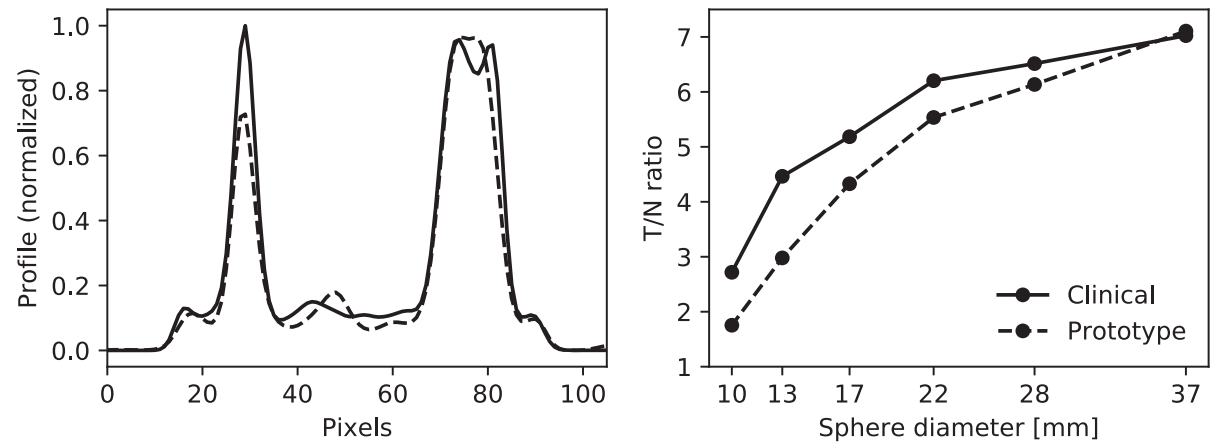

Figure 8: (a) The profile over the spheres of 22 and $17 \mathrm{~mm}$ diameter (see the white dotted line in Fig. 7), normalized to the total background activity. (b) $\mathrm{T} / \mathrm{N}$ ratios for all spheres present in the phantom. The spheres were filled with 10:1 uptake ratio.

the attenuation map generated by the clinical SPECT/CT. The patient bed was manually removed from the attenuation map for the reconstruction of the prototype scanner.

The SPECT reconstruction of the prototype scanner showed that the extrahepatic deposition was visible. The cold core was distinguishable, but had lower contrast than in the reconstruction of the clinical scanner. Some truncation was present toward the edges of the reconstruction, since the FOV of the gamma camera was smaller than that of the CBCT. Higher noise levels were observed in the liver background volume than in the reconstruction of the clinical scanner.

\section{Discussion}

A dual-layer detector that is capable of acquiring simultaneous fluoroscopic and nuclear projections was previously evaluated using planar imaging. This study evaluated via simulations and phantom experiments what image quality can be retrieved when performing SPECT. Although $\mathrm{CNR}$ and $\mathrm{T} / \mathrm{N}$ ratio were lower with the dual-layer scanner, the extrahepatic depositions of the anthropomorphic phantom were distinguished with this system. Furthermore, acquisition of simultaneous SPECT and CBCT was shown to be feasible. This could have advantages in a good integration in the intervention room due to the compact scanner size, intrinsically registered SPECT and CBCT reconstructions, and potential for respiratory motion correction. Acquiring information on the nuclear distribution during the intervention opens up a new area of research: in hepatic radioembolization, for instance, it could lead to single-session procedures.

A limitation of this study is that the LSF, often assessed in hepatic radioembolization, could not be measured with the phantom experiments due to the limited FOV of the prototype scanner. This evaluation was, however, performed in the simulation study, which showed that the LSF could be determined within 0.5 percent point, which can be considered clinically sufficient. Since the simulation study showed similar results as the phantom experiments, we are confident that we would be able to accurately determine the LSF in a system with larger FOV. 

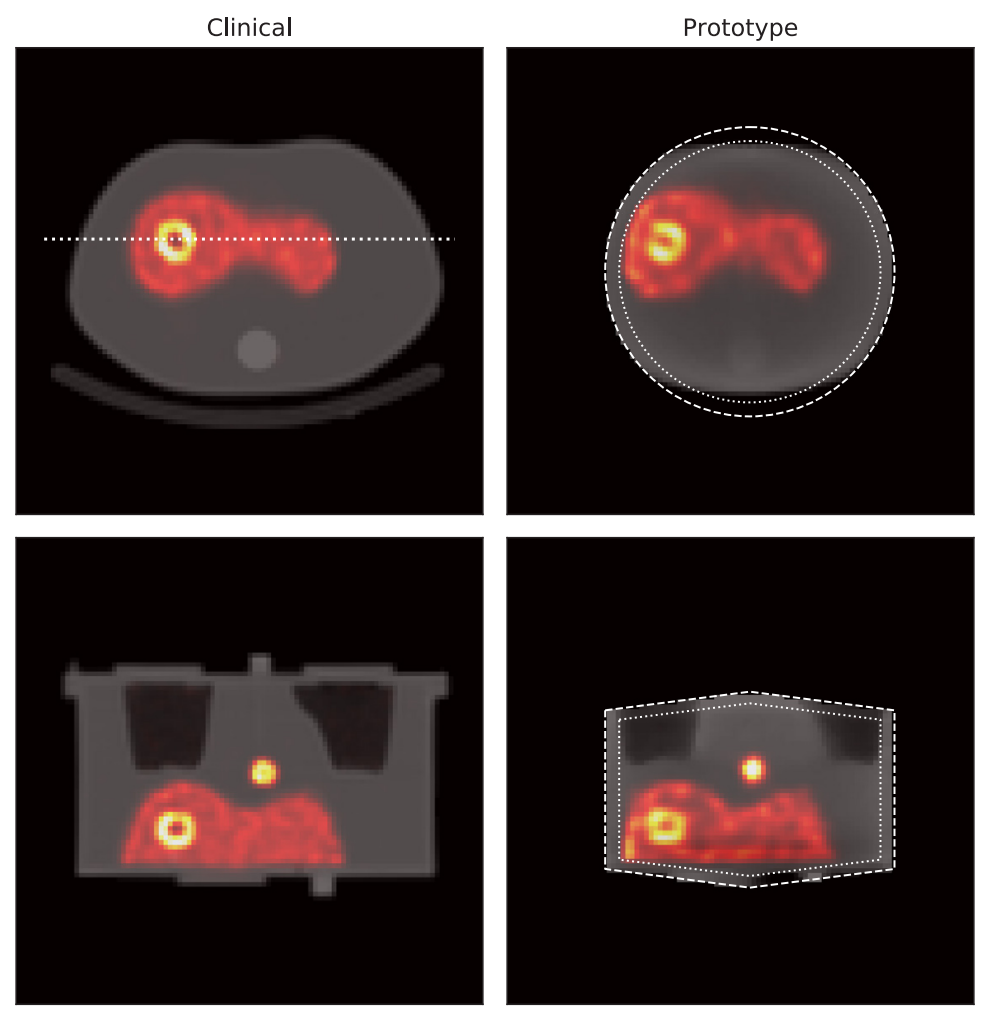

Figure 9: The reconstructions of the anthropomorphic phantom at iteration 10 for the clinical SPECT/CT (left) and the prototype SPECT/CBCT (right) of a transverse (top) and coronal (bottom) slice. The extrahepatic deposition in the shown slice had $15.7 \mathrm{ml}$ volume. The dotted white line in the upper-left figure denotes the position of the coronal slices. The dashed white lines in the right figures denote the field of view of the CBCT detector and the dotted lines denote the SPECT field of view.

A further limitation is that only extrahepatic depositions with volumes $(4.1,7.9$, and $15.7 \mathrm{ml})$ and activities (respectively $0.8 \%, 1.5 \%$, and $2.9 \%$ of the total injected activity) close to the median values as encountered in hepatic radioembolization $(6.8 \mathrm{ml}$ and $1.3 \%$ activity) were evaluated in this study. There is a large spread these values: in the study of Prince et al. [14], activities were found from 0.1 to $19.5 \%$ of the total injected activity and the volumes ranged from 1.1 to $42 \mathrm{ml}$. It might be that the dual-layer scanner will not able to detect the very small, low in activity extrahepatic depositions due to the decrease in visual image quality.

It will depend per procedure whether planar imaging with the dual-layer scanner is sufficient for feedback on the nuclear distribution or that a SPECT scan should be performed. In hepatic radioembolization, we envision that a physician could use planar imaging for determining the lung shunt fraction and to validate whether the correct liver segments are treated, and SPECT for detection of extrahepatic depositions, because of their smaller size. SPECT can, in contrast with planar imaging, also provide quantitative results, which can be beneficial for treatments in which the absolute administered activity is important. 
The fluoroscopic image quality of the dual-layer scanner will be lower than that of a conventional scanner, due to the removed anti scattergrid. Accordingly, we will perform the first step of the hepatic radioembolization procedure (i.e. vessel identification and catheter positioning) with a conventional CBCT scanner. The patient bed would be rotated after this step and the administration of activity would then be performed under guidance of the dual-layer scanner, whereupon a SPECT scan will be performed to evaluate the distribution in $3 \mathrm{D}$. In a later phase of the project, we will implement a scatter grid that aligns with the conebeam collimator so that the original CBCT quality might be restored without sacrificing SPECT quality.

The dual-layer scanner might be able to provide respiratory motion compensation, since nuclear and anatomical information is gathered simultaneously. The motion signal over time could be retrieved from the $\mathrm{CBCT}$ projections by tracking the lung-liver barrier. The corresponding motion vector field could be then obtained by registering gated $\mathrm{CBCT}$ reconstructions to a stationary frame, which could be included in the SPECT reconstruction. This approach was shown to work well in simulations [17]. The SPECT image quality improvement in phantom experiments will be studied in future work.

To be able to use the proposed scanner in an interventional setting, imaging should be performed quickly, ideally within minutes. The reduction in counts when scanning faster will increase noise in the reconstruction. In the case of hepatic radioembolization, however, quantitative measures as $\mathrm{T} / \mathrm{N}$ ratio are most important, which are less sensitive to noise. Recent work has shown that one can move towards a substantially reduced imaging time and still obtain quantitative results [18]. Furthermore, the possibility of acquiring images directly after injection will increase the number of counts as compared with a later imaging session at a clinical SPECT/CT. We expect that imaging within 10 minutes should be feasible.

Parameterized orbits require measurements of several distances to calculate the orbit from. We aim to perform these measurements automatically in the device under construction, so that potential miscalculations are avoided. A test rotation of approximately 30 seconds in duration will furthermore be performed before scanning to ensure that no collisions with the patient or bed occur. There is a large freedom in the configuration of orbits. If it appears that more general orbits (e.g. more elliptical) are required, these can be implemented without much effort. At a later stage in the project, the option to perform a body-tracing orbit can be added.

Similar to imaging speed, reconstructions should be performed within minutes to ensure a smooth workflow. In the current implementation, reconstruction takes approximately half an hour for 10 iterations with the mentioned image matrix size. With the use of multiprocessing, GPU-based matrix operations, and further code optimization, we expect that Monte Carlo-based reconstruction within 5 minutes should be achievable.

A different configuration, in which the flat panel is not positioned in front of the gamma camera (and thus is not reducing the gamma transmission and system resolution), could also be used for SPECT, for example using pinhole collimators as in a previous design [19]. However, a primary objective of the proposed scanner is to perform simultaneous planar imaging. This is more straightforwardly 
achieved with a dual-layer design, since both modalities intrinsically overlap. The dual-layer design furthermore admits of a compact detector that can be mounted on a custom C-arm. The scanner design could potentially be altered between planar imaging and SPECT during the procedure, but this would introduce extra operations and would limit practical use. This option has therefore not been further studied in this work.

The phantom experiments in this work cannot be directly compared with the results from the simulation study. The physical phantoms were positioned relatively close to the detector in the prototype scanner, in order to enlarge the reconstruction FOV. Such a situation cannot be achieved in a realistic setting, as the patient bed would get in the way. The patient bed would reduce sensitivity due to increased attenuation and reduce resolution due to a larger object-detector distance. The main goal of the experiments was to study the impact of performing simultaneous SPECT/CBCT, which has been shown not to hinder accurate nuclear imaging. We observed in the experiments no systematic artifacts from the removal of the anti scattergrid or the thinning of the back shielding on the fluoroscopic images.

The CBCT reconstruction with the prototype scanner were performed using low dose $(50 \mathrm{kVp}$ with $4 \mathrm{mAs}$ for the IQ phantom; $60 \mathrm{kVp}$ with $12 \mathrm{mAs}$ for the anthropomorphic phantom), because we want to limit the extra administered dose to patients. The above settings were found to be the minimum values where attenuation correction or registration could still be correctly performed. The IQ phantom required lower dose than the anthropomorphic phantom, because it is substantially smaller in size.

An increase in CBCT quality could be achieved by increasing the beam strength (both voltage and current). However, at higher beam strengths, the impact of $x$-ray pulses on the gamma camera will become substantial in the form of afterglow and photomultiplier-tube (PMT) limitations [20], which will severely reduce the fraction of usable gamma photons. Solutions for this limitation may be found in the modification of PMT circuitry by the introduction of a high-pass filter, mechanical shielding, or the use of different crystals for the gamma camera. For now, the obtained CBCT reconstructions are sufficiently accurate to perform attenuation correction or registration.

This work has focused on the visualization of ${ }^{99 \mathrm{~m}} \mathrm{Tc}$, because this is the most common isotope in SPECT and also used in the pre-treatment procedure of hepatic radioembolization. Eventually, we want to move towards imaging of the microspheres used in hepatic radioembolization treatment. These isotopes (e.g. ${ }^{90} \mathrm{Y}$ ) however radiate at higher energies, which requires thicker collimator septa and increased gamma camera shielding. Both have a major influence on the weight of the detector, complicating the design. For now, we concentrate on ${ }^{99 \mathrm{~m}} \mathrm{Tc}$ to facilitate a fast implementation in the clinic, but work is ongoing to also enable imaging of isotopes with higher energy.

Future work on the proposed dual-layer scanner will be on the development of the custom C-arm, so that the scanner can be used for patient studies. Additionally, it will be investigated whether the optimized flat panel can be manufactured. The gamma camera will be increased in size to enlarge the reconstruction FOV and changes to the PMT circuitry will be made, to cope with higher x-ray fluxes. 


\section{Conclusion}

Performing simultaneous SPECT/CBCT did not introduce artifacts in either the SPECT or the $\mathrm{CBCT}$ reconstructions for the studied situations. SPECT reconstruction quality of the dual-layer scanners was less than that of a clinical SPECT/CT scanner, but extrahepatic depositions with volumes and activities close to the median values encountered in hepatic radioembolization were distinguished from the background activity. The compact design of our system will allow inclusion of the scanner in intervention suite equipment and hence might improve interventional procedures involving radionuclides.

\section{References}

1. Ritt P, Sanders J, Kuwert T. SPECT/CT technology. Clin. Transl. Imaging 2(6):445-457 (2014)

2. Bailey DL, Willowson KP. Quantitative SPECT/CT: SPECT joins PET as a quantitative imaging modality. Eur. J. Nucl. Med. Mol. Imaging 41(1):17-25 (2014)

3. Bailey DL, Willowson KP. An Evidence-Based Review of Quantitative SPECT Imaging and Potential Clinical Applications. J. Nucl. Med. 54(1):83-89 (2013)

4. Kennedy A, Coldwell D, Sangro B, Wasan H, Salem R. Radioembolization for the treatment of liver tumors: General principles. Am. J. Clin. Oncol. Cancer Clin. Trials 35(1):91-99 (2012)

5. Braat AJAT, Smits MLJ, Braat MNGJA, et al. $Y^{90}$ Hepatic Radioembolization: An Update on Current Practice and Recent Developments. J. Nucl. Med. 56(2):1079-1087 (2015)

6. Gabr A, Kallini JR, Gates VL, et al. Same-day ${ }^{90} \mathrm{Y}$ radioembolization: implementing a new treatment paradigm. Eur. J. Nucl. Med. Mol. Imaging 43(13):2353-2359 (2016)

7. Garin E, Edeline J, Rolland Y. High Impact of Preferential Flow on ${ }^{99 \mathrm{~m}} \mathrm{Tc}-\mathrm{MAA}$ and ${ }^{90} \mathrm{Y}$-Loaded Microsphere Uptake Correlation. J. Nucl. Med. 57:1829-30 (2016)

8. Van der Velden S, Kunnen B, Koppert WJC, et al. A Dual Layer Detector for Simultaneous Fluoroscopic and Nuclear Imaging. Radiology 290(3):833-838 (2019)

9. Siemens, Symbia $S$ and T System Specifications. (2010)

10. Segars WP, Sturgeon G, Mendonca S, Grimes J, Tsui BMW. 4D XCAT phantom for multimodality imaging research. Med. Phys. 37(9):4902-4915 (2010)

11. De Jong HWAM, Slijpen ETP, Beekman FJ. Acceleration of Monte Carlo SPECT simulation using convolution-based forced detection. IEEE Trans. Nucl. Sci. 48(1):58-64 (2001)

12. Dietze MMA, Van der Velden S, Lam MGEH, Viergever MA, De Jong HWAM. Fast quantitative reconstruction with focusing collimators for liver SPECT. EJNMMI Phys. 5(1):28 (2018)

13. Jan S, Santin G, Strul D, et al. GATE: A simulation toolkit for PET and SPECT. Phys. Med. Biol. 49(19):4543-4561 (2004)

14. Prince JF, Van Rooij R, Bol GH, De Jong HWAM, Van den Bosch MAAJ, Lam MGEH. Safety of a Scout Dose Preceding Hepatic Radioembolization with ${ }^{166}$ Ho Microspheres. J. Nucl. Med. 56:817-23 (2015)

15. Biguri A, Dosanjh M, Hancock S, Soleimani M. TIGRE: a MATLAB-GPU toolbox for CBCT image reconstruction. Biomed. Phys. Eng. Express 2(5):055010 (2016)

16. Klein S, Staring M, Murphy K, Viergever MA, Pluim JPW. Elastix: A toolbox for intensity-based medical image registration. IEEE Trans. Med. Imaging 29(1):196-205 (2010)

17. Dietze MMA, Bastiaannet R, Kunnen B, Van der Velden S, Lam MGEH, Viergever MA, De Jong 
HWAM. Respiratory Motion Compensation in Interventional Liver SPECT using Simultaneous Fluoroscopic and Nuclear Imaging. Med. Phys. 46(8):3496-3507 (2019)

18. Van der Velden S, Dietze MMA, Viergever MA, De Jong HWAM. Fast technetium-99m liver SPECT for evaluation of the pre-treatment procedure for radioembolisation dosimetry. Med. Phys. 46(1):345355 (2019)

19. Beijst C, Elschot M, Viergever MA, De Jong HWAM. Toward Simultaneous Real-Time Fluoroscopic and Nuclear Imaging in the Intervention Room. Radiology 278(1):232-8 (2016)

20. Koppert WJC, Van der Velden S, Steenbergen JHL, De Jong HWAM. Impact of intense x-ray pulses on a $\mathrm{NaI}(\mathrm{Tl})$-based gamma camera. Phys. Med. Biol. 63(6):065006 (2018) 



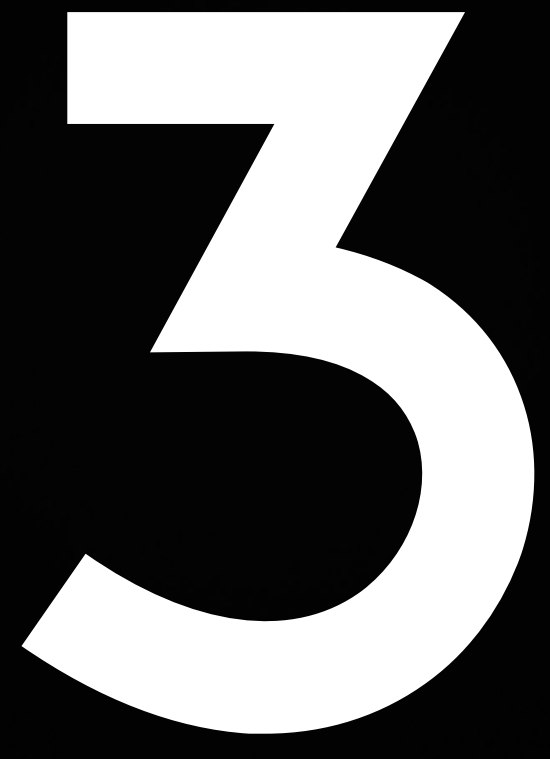




\section{A FULL-SCALE INTERVENTIONAL SCANNER}

BASED ON:

MMA Dietze, B Kunnen, F Brontsema, P Ramaekers, C Beijst, M Afifah, AJAT Braat, MGEH Lam, HWAM de Jong

A Compact and Mobile Hybrid C-arm Scanner for Simultaneous Nuclear and Fluoroscopic Image Guidance 


\begin{abstract}
Background

Current hybrid SPECT/CT scanners are bulky and acquire $\mathrm{x}$-ray and nuclear images in sequence, which hinders the integration into the intervention room and dynamic imaging.

\section{Purpose}

This study evaluates the performance and demonstrates the potential applications of a mobile and compact hybrid c-arm scanner (referred to as IXSI) that is capable of simultaneous fluoroscopic and nuclear projection acquisition with the same field of view and which can be easily introduced in the intervention room.
\end{abstract}

\title{
Materials and Methods
}

Two applications of IXSI were demonstrated: i) simultaneous acquisition of planar (2D) fluoroscopic and nuclear projections (for procedure guidance of interventional procedures involving the administration of radionuclides), and ii) acquisition of SPECT/CBCT (3D) reconstructions (for a quantitative evaluation of radionuclide distributions inside the intervention room). The reconstruction quality of IXSI was compared with that of a clinical SPECT/CT scanner. The impact of slightly misaligning the IXSI modalities (in an off-focus geometry) was investigated for the reduction of the $\mathrm{x}$-ray and nuclear interference for simultaneous imaging.

\section{Results}

A phantom experiment in which a mixture of ${ }^{99} \mathrm{~m} T \mathrm{c}$ and iodinated contrast was slowly injected into an anthropomorphic phantom during simultaneous acquisition of nuclear and fluoroscopic projections simulated the procedure guidance capability of IXSI for interventions involving radionuclides. The activity recoveries of the NEMA spheres obtained by the SPECT/CBCT scan of IXSI showed $<8 \%$ difference compared with those obtained by the clinical scanner. The off-focus geometry improved the maximum tolerable $\mathrm{x}$-ray tube beam strength for simultaneous imaging from $80 \mathrm{kVp}$ to $>100 \mathrm{kVp}$.

\section{Conclusion}

A mobile and compact hybrid c-arm scanner, capable of simultaneous fluoroscopic and nuclear projection acquisition with the same field of view, accomplishes unique image guidance and may benefit interventional procedures involving radionuclides. 


\section{Introduction}

Fluoroscopy-guided procedures involving radionuclides (e.g. hepatic radioembolization [1] and sentinel node procedures [2]) would benefit from nuclear imaging performed in the intervention room by allowing a direct evaluation of the activity distribution. Such interventional imaging may result in more personalized treatments and could introduce an easier one-stop-shop treatment approach.

Current hybrid SPECT/CT scanners are bulky and acquire $\mathrm{x}$-ray and nuclear images in sequence, which hinders dynamic imaging and the integration into the intervention room. A detector configuration that meets the requirements for use in the intervention room was previously proposed and evaluated in prototype systems [3-5]. The current study evaluates the first scanner that has been approved for clinical use.

\section{Materials and methods}

\section{IXSI}

The developed scanner will be referred to as IXSI (Interventional X-ray and Scintigraphy Imaging). The detector stack of IXSI (see Fig. 1a) consists of an x-ray flat panel detector (Pixium 3040; Trixell, Moirans, France) that is positioned in front of a gamma camera (P3000; Inter Medical, Lübbecke, Germany) that is mounted with a cone beam collimator (Nuclear Fields, VortumMullem, The Netherlands). The detector stack is placed on a custom-made c-arm (Indes, Enschede, The Netherlands) together with an $\mathrm{x}$-ray tube (Veradius; Philips Healthcare, Best, The Netherlands) on the opposite side. The $\mathrm{x}$-ray tube is placed in the focal point of the cone beam collimator (105 $\mathrm{cm}$ ), which results in simultaneously overlapping nuclear and fluoroscopic projections (see Fig. 1b). The c-arm can translate in two dimensions while rotating and is able to perform parameterized non-circular orbits. A collision detector is present on top of the detector stack to ensure movement termination upon contact with an object.

The flat panel detector module was, with the help of Philips Healthcare (Amsterdam, The Netherlands), adjusted from the commercial product by the reduction of the back-shielding thickness and the removal of highly attenuating objects (e.g. support screws). This adjusted flat panel detector module has a thickness of $6.8 \mathrm{~cm}$, an average gamma transmission of $52 \%$ at $140 \mathrm{keV}$, and a field of view (FOV) of $39.9 \times 29.5 \mathrm{~cm}^{2}$. The cone beam collimator has holes with $40.0 \mathrm{~mm}$ length, $1.90 \mathrm{~mm}$ inner diameter, and $0.25 \mathrm{~mm}$ septal thickness. The gamma camera has a $3.9 \mathrm{~mm}$ full width at half maximum (FWHM) intrinsic spatial resolution, a $9.4 \%$ energy resolution (at 140 $\mathrm{keV}$ ), and a $51.0 \times 38.1 \mathrm{~cm}^{2}$ effective FOV.

\section{Nuclear and $\mathrm{x}$-ray interference}

The majority of the $x$-ray photons is absorbed by the flat panel detector when imaging simultaneously. However, a fraction penetrates the flat panel detector to be absorbed by the gamma camera. Previously, it was demonstrated that this $\mathrm{x}$-ray spillover effect does not influence the gamma camera performance for dose levels of up to $80 \mathrm{kVp}$ (at $1.17 \mathrm{~mA}$ ) [6,7]. However, since the fluoroscopic 

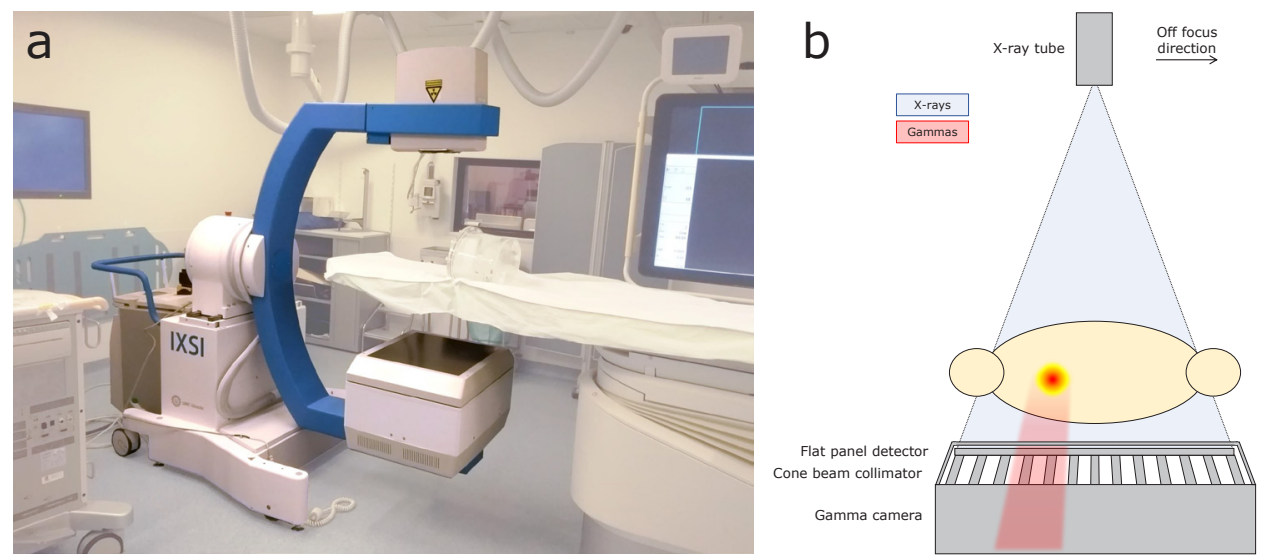

Figure 1: (a) IXSI in the intervention room. (b) A schematic illustration of the IXSI scanner components. For the in-focus configuration, the collimator holes are aligned with the $\mathrm{x}$-ray focal point. For the off-focus configuration, the $\mathrm{x}$-ray tube is shifted by $5 \mathrm{~cm}$ in the indicated direction.

protocols in our institute are often performed with higher beam strengths, a higher maximum tolerable beam strength may be beneficial.

In this work, we propose to translate the $\mathrm{x}$-ray tube off-focus from the focal point of the cone beam collimator by $5 \mathrm{~cm}$ (see Fig. 1b). This translation introduces a distance-dependent mismatch between the fluoroscopic and nuclear projections but has the advantage that $\mathrm{x}$-ray spillover is greatly reduced (due to the increased collimator attenuation). Two experiments were conducted to study these effects.

\section{Registration mismatch}

Three ${ }^{57}$ Co pen markers (1.2 MBq each) were positioned 5, 15, and $25 \mathrm{~cm}$ in front of the detector stack. Nuclear projections (measured for 2 minutes and smoothed with a $5 \mathrm{~mm}$ FWHM Gaussian filter) and fluoroscopic projections were acquired with the $\mathrm{x}$-ray tube positioned: $\mathrm{i}$ ) in the focal point of the cone beam collimator, and ii) off-focus by $5 \mathrm{~cm}$. The centers of the pen markers were determined in both projections and the mismatch was quantified.

\section{$X$-ray spillover}

A $350 \mathrm{MBq}{ }^{99 \mathrm{~m}} \mathrm{Tc}$ point source was placed on the back of an anthropomorphic phantom (to create a realistic scatter situation). Nuclear projections were acquired in list-mode and the gamma count rate in the $140 \pm 7.5 \% \mathrm{keV}$ energy window was determined. Fluoroscopic projections ( $27.8 \mathrm{~ms}$ pulse length, $3.75 \mathrm{~Hz}$ ) were then acquired from 70 to $100 \mathrm{kVp}(1.14$ to $1.22 \mathrm{~mA})$ while the gamma count rate in the same energy window was again determined (for the intervals in which the $\mathrm{x}$-ray tube was not active). The usable gamma data fraction, as a function of the $\mathrm{x}$-ray tube strength, was calculated by dividing these two count rates. 
a

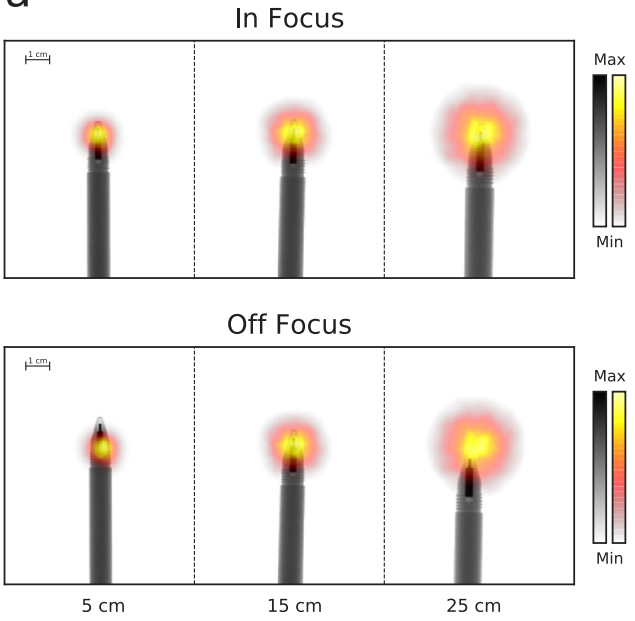

b

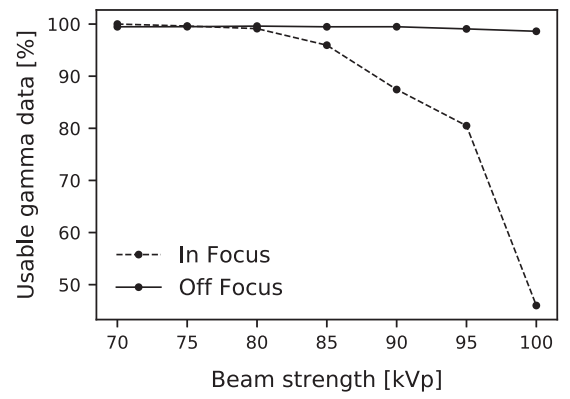

Figure 2: (a) The fluoroscopic (in gray-scale) and nuclear (in color) projections obtained with the in- and off-focus x-ray tube configurations. (b) The usable gamma data when simultaneously acquiring nuclear and fluoroscopic projections, as a function of beam strength, for the in- and off-focus x-ray tube configuration.

\section{Scanner comparison}

The detector characteristics of IXSI were compared to those of a clinical SPECT/CT system (Symbia T16; Siemens Healthineers, Erlangen, Germany). The clinical scanner had two detector heads that were mounted with parallel-hole low-energy high-resolution (LEHR) collimators (24.05 mm hole length, $1.11 \mathrm{~mm}$ inner diameter, and $0.16 \mathrm{~mm}$ septal thickness) and had a $3.9 \mathrm{~mm}$ FWHM intrinsic spatial resolution, a $9.9 \%$ energy resolution (at $140 \mathrm{keV}$ ), and a $53.3 \times 38.7 \mathrm{~cm}^{2}$ effective FOV.

The detector spatial resolution and sensitivity were determined. A ${ }^{99} \mathrm{~m} T \mathrm{Tc}$ point source $(30 \mathrm{MBq})$ was positioned at distances from 5 to $40 \mathrm{~cm}$ to the detector (in steps of $5 \mathrm{~cm}$ ) and measured for one minute. The detector spatial resolution was determined by measuring the FWHM of the Gaussian functions fitted to the projections (for IXSI, divided by the projection magnification from the cone beam collimator). The detector sensitivity was determined by dividing the total obtained counts by the calibrated source activity.

\section{Applications}

Two applications of IXSI were demonstrated with phantom experiments: simultaneous planar imaging and SPECT/CBCT imaging.

\section{Simultaneous planar imaging}

An empty syringe of $20 \mathrm{ml}$ was placed in an anthropomorphic phantom and during 40 seconds filled with a mixture of iodinated contrast and $50 \mathrm{MBq}{ }^{99 \mathrm{~m}} \mathrm{Tc}$ via a connecting tube (resembling an injection during the pre-treatment procedure of hepatic radioembolization). The fluoroscopic 

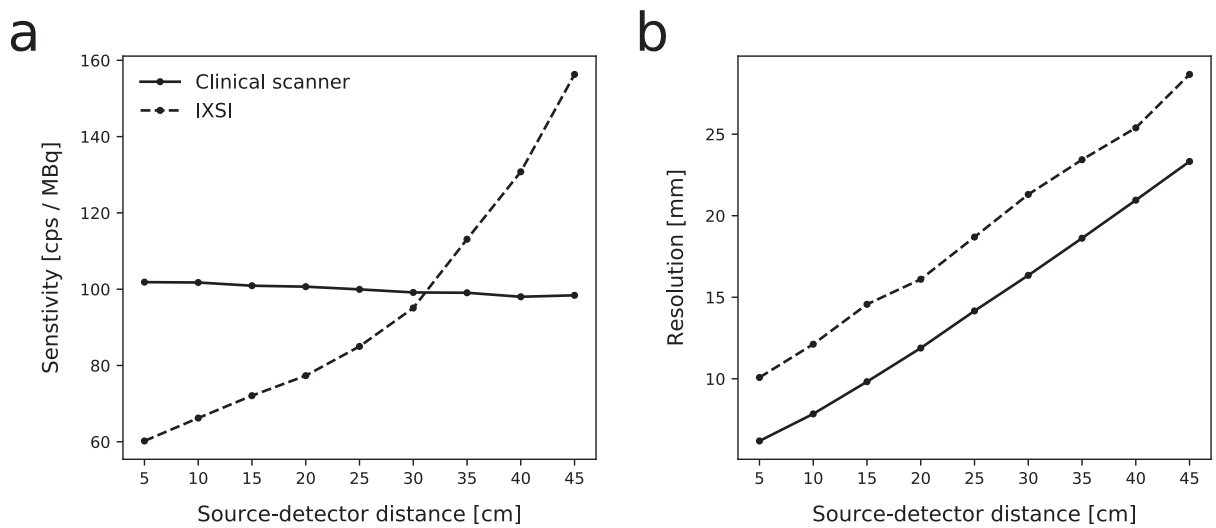

Figure 3: (a) The detector sensitivity and (b) the spatial resolution, as a function of the distance from the source to the detector, for IXSI and one detector head of the clinical scanner.

projections were acquired at $80 \mathrm{kVp}(27.8 \mathrm{~ms}$ pulse length, $3.75 \mathrm{~Hz}, 1.17 \mathrm{~mA})$. The nuclear projections were created with an integration time of 2.7 seconds and were smoothed with a $5 \mathrm{~mm}$ FWHM Gaussian filter. Digital subtraction images were created by subtracting the fluoroscopic projections obtained during the injection with those acquired before the start of the injection.

\section{SPECT/CBCT imaging}

The NEMA image quality phantom $\left(150 \mathrm{MBq}{ }^{99 \mathrm{~m}} \mathrm{Tc}\right.$ total activity; the spheres filled at an 8:1 concentration ratio) was scanned on both IXSI and the clinical scanner for 10 minutes over 360 degrees with 120 angles. Both scanners rotated closely around the phantom. The scans were repeated 5 times (with the acquisition times adjusted to account for radioactive decay) to assess the stability of the reconstructions.

For the clinical scanner, the attenuation map was retrieved from the clinical software after performing a low-dose CT (made with $110 \mathrm{kVp}$ at $14 \mathrm{mAs}$ ). For IXSI, the attenuation map was retrieved by registering the CBCT reconstruction (made with $80 \mathrm{kVp}$ at $73 \mathrm{mAs}$ ) to the attenuation map of the clinical scanner using the elastix software package [8]. The registration was required for two reasons. First, the CBCT reconstruction quality suffered from scattered photons, which may affect the quantitative accuracy of the SPECT reconstruction. And second, the CBCT reconstruction FOV is limited, which results in an incomplete attenuation map when larger objects are imaged.

The nuclear projections were, for both scanners, reconstructed with the Utrecht Monte Carlo System iterative reconstruction software package, which includes attenuation compensation, resolution recovery, and Monte Carlo-based scatter correction [9,10]. The projections were reconstructed on a $128 \times 128 \times 128$ grid with a $4.79 \mathrm{~mm}$ isotropic voxel size. The reconstructor used the OSEM reconstruction algorithm ( 6 iterations with 8 subsets) and the reconstructions were smoothed with a $5 \mathrm{~mm}$ FWHM Gaussian filter. The activity recoveries of the spheres in the NEMA image quality phantom (delineated on the low-dose CT reconstruction) were determined as a measure of the reconstruction quality. 

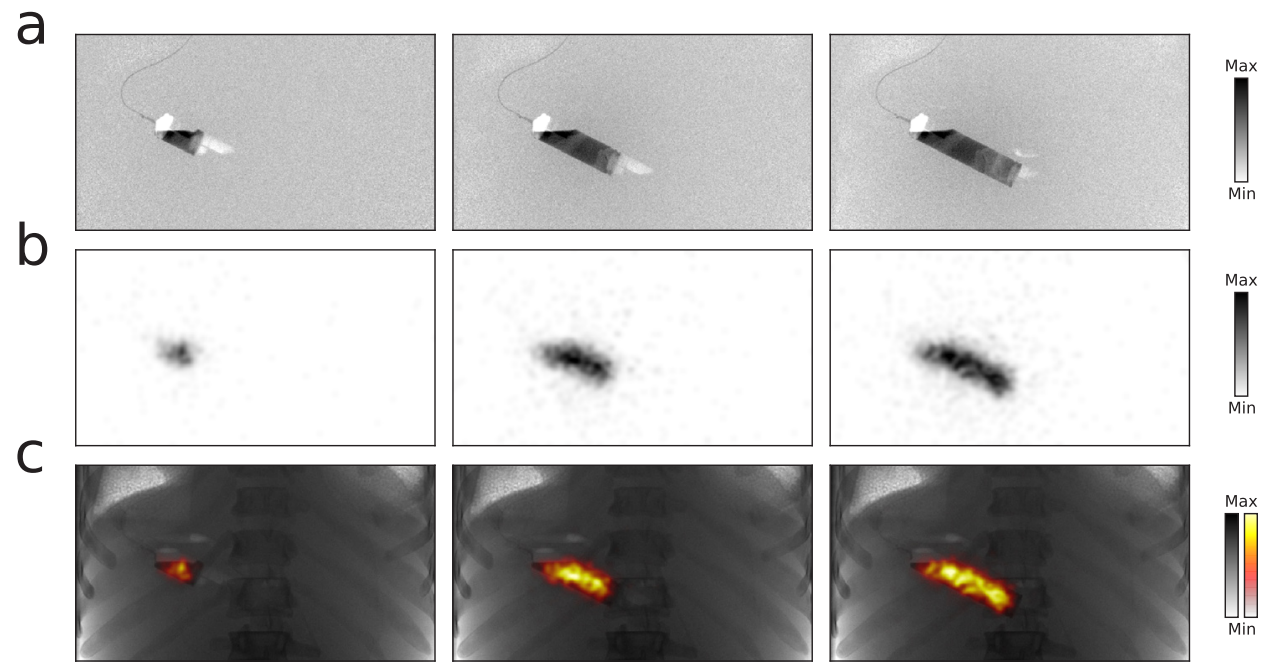

Figure 4: The projections at three time points (15, 30, and 40 seconds from the start of the injection) that are obtained during the injection of iodinated contrast with ${ }^{99 \mathrm{~m}} \mathrm{Tc}$. Shown are (a) the digital subtraction images, (b) the nuclear projections, and (c) the fluoroscopic projections (in gray-scale) merged with the nuclear projections (in color).

\section{Results}

\section{Nuclear and $x$-ray interference}

The fluoroscopic and nuclear projections of the point sources obtained with the in- and off-focus $\mathrm{x}$-ray tube configuration are shown in Fig. 2a. The scanner was calibrated such that the nuclear and fluoroscopic projections overlapped at $15 \mathrm{~cm}$ from the detector. The resulting mismatch was $<1 \mathrm{~cm}$ for the pen markers positioned at 5 and $25 \mathrm{~cm}$ from the detector.

The usable data fraction of the gamma camera, as a function of the $\mathrm{x}$-ray tube beam strength, is shown in Fig. $2 \mathrm{~b}$ for both $\mathrm{x}$-ray tube configurations. The maximum beam strength at which no gamma data loss occurred increased from 80 to $>100 \mathrm{kVp}$ (the limit of the $\mathrm{x}$-ray tube) when moving from the in-focus to the off-focus configuration. The off-focus configuration was hence used in the remainder of this work.

\section{Scanner comparison}

The detector sensitivity and resolution, as a function of the distance of the point source to the detector, are shown in Fig. 3 for IXSI and the clinical scanner. IXSI had a lower sensitivity than the clinical scanner due to the increased attenuation from the flat panel detector module, which increased over the source to detector distance due to the converging nature of the cone beam collimator. IXSI had a worse spatial resolution than the clinical scanner (consistently $5 \mathrm{~mm}$ larger) due to the extra source to collimator distance arising from the thickness of the flat panel detector module. 

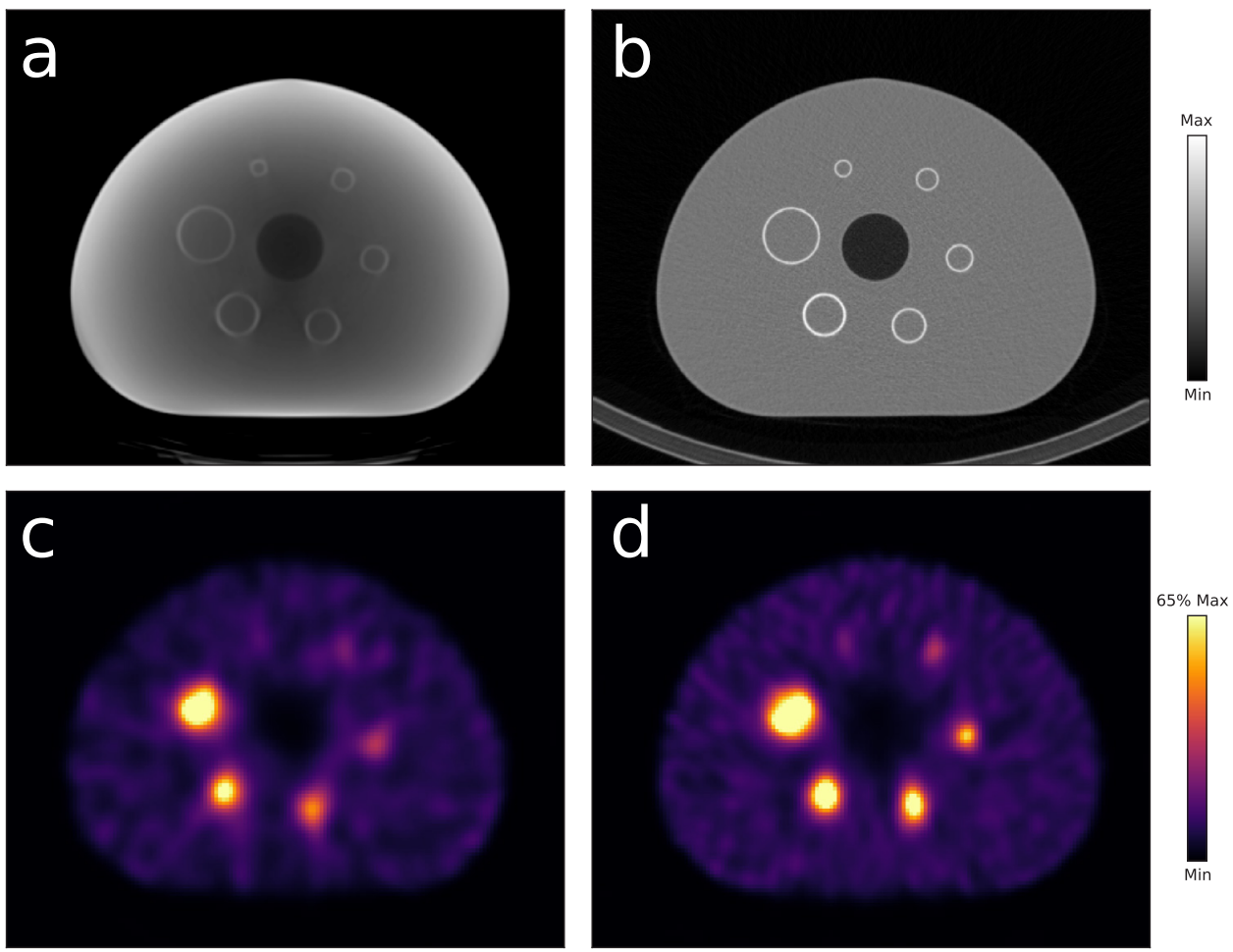

Figure 5: (a) The CBCT reconstruction from IXSI. (b) The low-dose CT reconstruction from the clinical scanner. (c) The SPECT reconstruction from IXSI. (d) The SPECT reconstruction from the clinical scanner.

\section{Applications}

\section{Simultaneous planar imaging}

The nuclear, fluoroscopic, and digital subtraction projections obtained during the injection of iodinated contrast with ${ }^{99 \mathrm{~m}} \mathrm{Tc}$ are shown at three time points in Fig. 4.

\section{SPECT/CBCT imaging}

Slices of the SPECT and (CB)CT reconstructions of the NEMA image quality phantom are shown in Fig. 5. The clinical scanner acquired $(5.38 \pm 0.02) \times 10^{6}$ nuclear counts, while IXSI acquired $(2.25$ $\pm 0.01) \times 10^{6}$ counts. The activity recoveries of the NEMA image quality phantom spheres are given in Tab. 1 . The activity recoveries from IXSI showed $<8 \%$ difference compared with those obtained by the clinical scanner.

\section{Discussion}

This study presented the evaluation of a hybrid c-arm scanner and demonstrated its potential applications. The combination of $\mathrm{x}$-ray and nuclear detection capability in one detector allows for a compact scanner that is compatible with a c-arm which enables a smooth implementation in 
Table 1: The activity recoveries (in percentage) of the NEMA image quality phantom spheres for IXSI and the clinical scanner.

\begin{tabular}{c|cc} 
Sphere diameter $[\mathrm{mm}]$ & IXSI & Clinical scanner \\
\hline 37 & $72.7 \pm 0.9$ & $74.2 \pm 0.9$ \\
28 & $61.7 \pm 1.1$ & $68.2 \pm 1.0$ \\
22 & $50.4 \pm 4.7$ & $53.3 \pm 2.1$ \\
17 & $37.2 \pm 2.8$ & $44.5 \pm 4.8$ \\
13 & $25.6 \pm 2.9$ & $31.2 \pm 1.7$ \\
10 & $17.9 \pm 2.5$ & $18.8 \pm 2.1$
\end{tabular}

the intervention room. The ability to simultaneously gather information on the anatomy and the activity distribution allows for unique image guidance of (dynamic) radionuclide procedures.

The off-focus geometry introduced a distance-dependent mismatch between the fluoroscopic and the nuclear projections. This mismatch limits the simultaneous image guidance of small activity accumulations. However, when imaging relatively larger accumulations (e.g. in the planar imaging phantom experiment), the average mismatch is comparable with the collimator resolution and the interpretation of the projections is not substantially affected.

The noise in the SPECT reconstruction of IXSI was substantially higher than that of the clinical scanner due to the lower number of detected counts. It has, however, previously been demonstrated that quantitative SPECT measures in such high-noise reconstructions can often be accurately extracted [11-13].

A further application of the simultaneous acquisition of fluoroscopic and nuclear projections lies in the potential for motion compensation. This methodology has been studied with digital simulations and substantially improved the quantitative accuracy of the SPECT reconstructions [14].

\section{References}

1. Braat AJAT, Smits MLJ, Braat MNGJA, et al. ${ }^{90}$ Y Hepatic Radioembolization: An Update on Current Practice and Recent Developments. J Nucl Med 2015;56(7):1079-1087

2. Vidal-Sicart S, Paredes P, Zanón G, Pahisa J, Martinez-Román S, Caparrós X, Vilalta A, Rull R, Pons F. Added value of intraoperative real-time imaging in searches for difficult-to-locate sentinel nodes. J Nucl Med. 2010;51(8):1219-25

3. Beijst C, Elschot M, Viergever MA, De Jong HWAM. Toward Simultaneous Real-Time Fluoroscopic and Nuclear Imaging in the Intervention Room. Radiology 2016;278(1):232-238

4. Van der Velden S, Kunnen B, Koppert WJC, et al. A Dual-layer Detector for Simultaneous Fluoroscopic and Nuclear Imaging. Radiology 2019;290(3):833-838

5. Dietze MMA, Kunnen B, Van der Velden S, Steenbergen JHL, Koppert WJC, Viergever MA, De Jong HWAM. Performance of a dual-layer scanner for hybrid SPECT/CBCT. Phys Med Biol 2019;64(10):105020

6. Koppert WJC, Van der Velden S, Steenbergen JHL, De Jong HWAM. Impact of intense x-ray pulses on a NaI(Tl)-based gamma camera. Phys Med Biol 2018;63(6):065006 
7. Koppert WJC, Dietze MMA, Van der Velden S, Steenbergen JHL, De Jong HWAM. A comparative study of $\mathrm{NaI}(\mathrm{Tl}), \mathrm{CeBr}_{3}$, and $\mathrm{CZT}$ for use in a real-time simultaneous nuclear and fluoroscopic dual-layer detector. Phys Med Biol 2019;64(13):135012

8. Klein S, Staring M, Murphy K, Viergever MA, Pluim JP. elastix: A Toolbox for Intensity-Based Medical Image Registration. IEEE Trans Med Im 2009; 29(1):196-205

9. De Jong HWAM, Slijpen ETP, and Beekman FJ, Acceleration of Monte Carlo SPECT simulation using convolution-based forced detection, IEEE Trans. Nucl. Sci. 2011; 48(1) 58-64

10. Dietze MMA, Van der Velden S, Lam MGEH, Viergever MA, De Jong HWAM, Fast quantitative reconstruction with focusing collimators for liver SPECT, EJNMMI Phys. 2018; 5(1) 28

11. Van der Velden S, Dietze MMA, Viergever MA, De Jong HWAM. Fast technetium-99m liver SPECT for evaluation of the pretreatment procedure for radioembolization dosimetry. Med Phys 2019;46(1):345355

12. Dietze MMA, Kunnen B, Beijst C, De Jong HWAM. Adaptive Scan Duration in SPECT: Evaluation for Radioembolization. Med Phys 2020; 47(5) 2128-2138

13. Kunnen B, Dietze MMA, Braat AJAT, Lam MGEH, Viergever MA, de Jong HWAM, Feasibility of imaging ${ }^{90} \mathrm{Y}$ microspheres at diagnostic activity levels for hepatic radioembolization treatment planning, Med Phys 2019; 47(3) 1105-1114

14. Dietze MMA, Bastiaannet R, Kunnen B, Van der Velden S, Lam MGEH, Viergever MA, De Jong HWAM. Respiratory Motion Compensation in Interventional Liver SPECT using Simultaneous Fluoroscopic and Nuclear Imaging. Med Phys 2019;46(8):3496-3507 



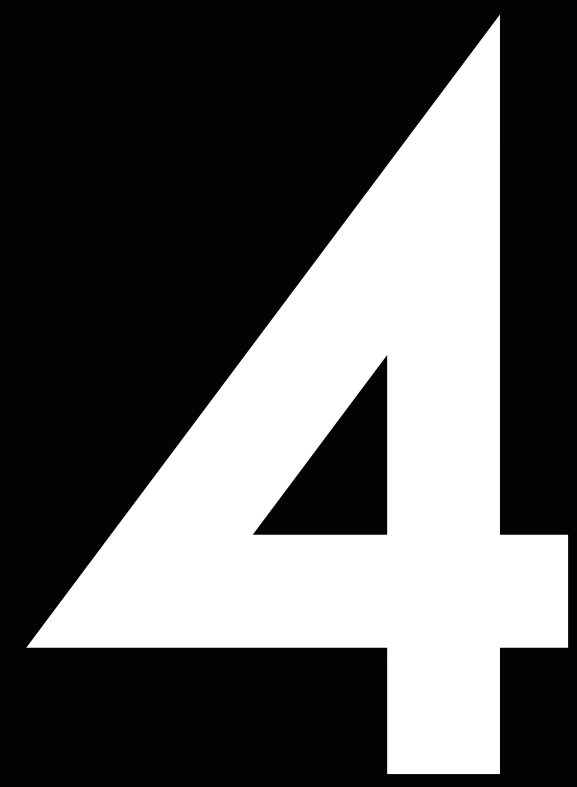




\section{NUCLEAR IMAGING WITH AN X-RAY FLAT PANEL DETCTOR}

PUBLISHED AS:

MMA Dietze, WJC Koppert, R van Rooij, HWAM de Jong Nuclear Imaging with an X-Ray Flat Panel Detector: A Proof-of-Concept Study 


\begin{abstract}
Purpose

Interventional procedures involving radionuclides (e.g. radioembolization) would benefit from SPECT performed in the intervention room because the activity distribution could be immediately visualized. We believe it might be possible to perform SPECT with the C-arm CBCT scanner present in the intervention room by equipping the $\mathrm{x}$-ray flat panel detector with a collimator. The purpose of this study is to demonstrate the approach and to investigate the achievable SPECT reconstruction quality.
\end{abstract}

\title{
Methods
}

A proof-of-concept experiment was performed to evaluate the possibility of nuclear imaging with an $\mathrm{x}$-ray flat panel detector. The experiment was digitally replicated to study the accuracy of simulations. Three flat panel configurations (with standard hardware and reconstruction methodology, with sophisticated reconstruction methodology, and with expected future hardware) and a conventional gamma camera were evaluated. The Jaszczak and the NEMA IQ phantom (filled with ${ }^{99 \mathrm{~m}} \mathrm{Tc}$ ) were simulated and assessed on resolution and contrast-to-noise ratio (CNR).

\section{Results}

The proof-of-concept experiment demonstrated that nuclear images could be obtained from the flat panel detector. The simulation of the same configuration demonstrated that simulations could accurately predict the flat panel detector response. The CNR of the $37 \mathrm{~mm}$ sphere in the NEMA IQ phantom was $22.8 \pm 1.2$ for the gamma camera reconstructions, while it was $11.3 \pm 0.7$ for the standard flat panel detector. With sophisticated reconstruction methodology, the CNR improved to $13.5 \pm 1.4$. The CNR can be expected to advance to $18.1 \pm 1.3$ for future flat panel detectors.

\section{Conclusion}

The x-ray flat panel detector of a CBCT scanner might be used to perform nuclear imaging. The SPECT reconstruction quality will be lower than that achieved by a conventional gamma camera. The flat panel detector approach could, however, be useful in providing a cost-effective alternative to the purchase of a mobile SPECT scanner for enabling interventional scanning. 


\section{Introduction}

Interventional procedures involving radionuclides (e.g. radioembolization) would benefit from single-photon emission computed tomography (SPECT) performed in the intervention room because this would allow the activity distribution to be immediately visualized. Work is ongoing on the development of mobile and compact SPECT scanners [1-3]. However, the associated costs of a new scanner might raise a threshold for widespread application in clinical practice. It would be beneficial if a method for interventional SPECT scanning is developed which does not require the purchase of an additional scanner.

A scanner that is usually already available in the intervention room is the C-arm cone beam computed tomography (CBCT) scanner, which is used e.g. for fluoroscopic guidance in $2 \mathrm{D}$ and position verification in 3D [4]. Although the CBCT scanner design is optimized for imaging of $\mathrm{x}$-rays, its flat panel detector should also be able to detect gamma photons when equipped with a collimator. The difference with a conventional gamma camera is that the detecting efficiency of the flat panel detector will be much lower and that currently no photon counting and thus photon energy selection can be performed; its intrinsic spatial resolution is, however, much better.

Due to the lower detector sensitivity, the SPECT reconstructions from a flat panel detector can be expected to be noisier than those from a conventional gamma camera. We propose a combination of two methods to boost the flat panel detector sensitivity. First, since the flat panel detector has an excellent intrinsic spatial resolution, the reconstruction resolution can be improved by incorporating a detailed collimator model. This methodology has been shown to be feasible in other studies utilizing small pixel size detectors [5-8]. Second, the improved resolution can be traded with a higher sensitivity by enlarging the collimator hole width. The combination of these methods should be able to improve the reconstruction quality.

This study starts by illustrating the feasibility of nuclear imaging with a flat panel detector using a proof-of-concept experiment. Using simulations, the achievable reconstruction quality of a flat panel detector is evaluated and compared with that of a conventional gamma camera. It is studied whether the combination of detailed collimator modeling and enlarged collimator holes provides improvements to the reconstruction quality. Finally, the reconstruction quality of future flat panel detectors (which are expected to possess the capability for photon energy selection $[9,10]$ ) is assessed.

\section{Methods}

\section{Proof-of-concept experiment}

An experiment was performed to illustrate the feasibility of nuclear imaging with an $\mathrm{x}$-ray flat panel. A parallel-hole collimator (29.1 mm hole length, $2.49 \mathrm{~mm}$ hole width, $0.50 \mathrm{~mm}$ septal thickness) was positioned directly in front of a commercially available flat panel detector with $0.308 \mathrm{~mm}$ isotropic pixel size (Pixium 3040; Trixell, Moirans, France). The flat panel detector used a thin CsI crystal for scintillation and had an energy-integrating readout. A bottle (12 mm inner radius) filled 
Gamma Camera
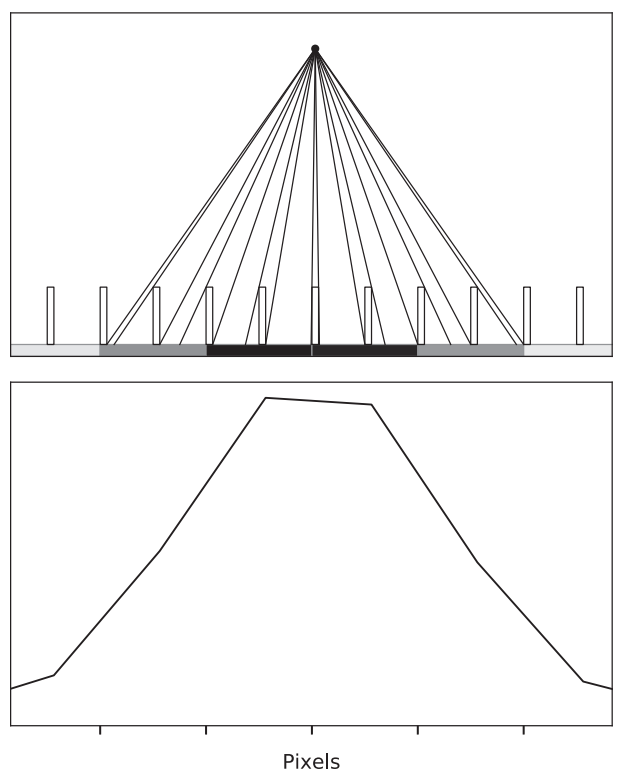

Flat Panel
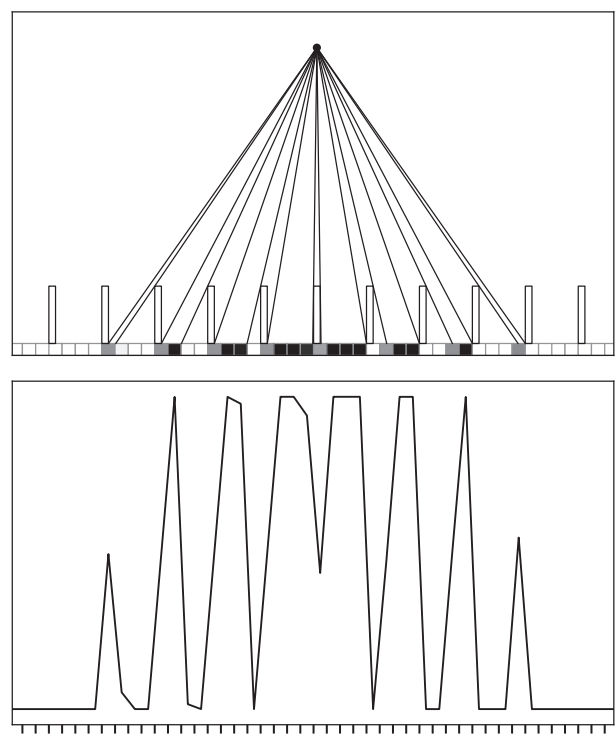

Pixels

Figure 1: The profiles obtained from a point source positioned $10 \mathrm{~cm}$ in front of the collimator for a conventional gamma camera and a flat panel detector. The black lines in the top figures denote the optical transmission of photons through the collimator.

with $414 \mathrm{MBq}{ }^{99 \mathrm{~m}} \mathrm{Tc}$ was positioned $12 \mathrm{~cm}$ in front of the flat panel detector. A projection was made with a measurement time of 50 seconds.

The experiment configuration was then digitally replicated in GATE [11] (with photoelectric effect, Compton scatter, and Rayleigh scatter in the source and the collimator) to study to what extent the simulations can accurately predict the flat panel detector response. The acquired projections from the real data and the simulation were compared on their shape and size.

\section{Detailed collimator modeling}

Figure 1 illustrates the projection geometry of a point source positioned $10 \mathrm{~cm}$ in front of a lowenergy parallel hole collimator $(24.05 \mathrm{~mm}$ hole length, $1.077 \mathrm{~mm}$ hole width, $0.155 \mathrm{~mm}$ septal thickness) for a conventional gamma camera (with a pixel size of $2.464 \mathrm{~mm}$ ) and a flat panel detector (pixel size of $0.308 \mathrm{~mm}$ ). The profile of the flat panel detector projection is very different from that of the conventional gamma camera because the small pixel size allows to resolve patterns that are smaller than the distance between the septa. Additional resolution information is retrieved from the position and the magnitude of the peaks present in the detector profile.

To take the additional resolution information fully into account in the reconstruction, accurate point spread function (PSF) models are required in both the forward projection of the reconstructor (i.e. given a source in the reconstruction space, this is the resulting profile on the detector) as well as the 


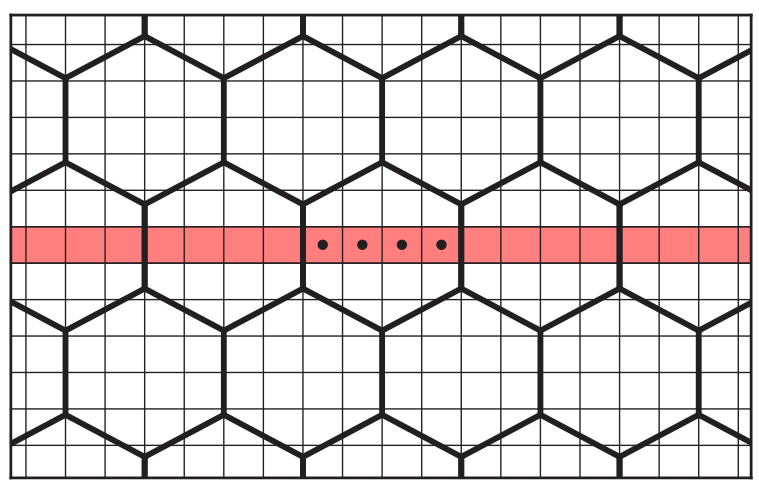

Figure 2: Top view of the custom LEHR collimator in front of the flat panel detector pixels. The black dots denote the locations where the PSF models are sampled. Marked in red is the slice that is used in the $2 \mathrm{D}$ reconstruction.

backward projection (i.e. given a count on the detector, these are the locations in the reconstruction space where it could have originated from). Practically, this means that the PSF models used in the reconstructor should be a function of the relative position above the collimator septum (in addition to being depth-dependent). We will refer to this method as 'detailed collimator modeling.

\section{Point spread function generation}

The detailed collimator modeling requires a separate PSF model for every individual pixel in the reconstruction space, which is computationally demanding to simulate. To reduce the number of samples required, we will make use of the repeating pattern of a parallel-hole collimator. A simulated low-energy high-resolution (LEHR) collimator (hole width of $1.270 \mathrm{~mm}$ [12]) had its dimensions slightly reduced to obtain a hole width of $1.232 \mathrm{~mm}$ (this resized collimator will be referred to as the 'custom LEHR'). Exactly four detector pixels (with a size of $0.308 \mathrm{~mm}$ ) now fit into a collimator hole (see Fig. 2). Only those pixels need to be sampled since the remainder of the space is covered by repetition (see the black dots in Fig. 2).

Reconstructions were performed in 2D (rather than 3D) to reduce the computation time even further. Only the slice that is positioned over the middle of the collimator holes (see the pixels marked in red in Fig. 2) was used in the reconstruction and hence only the PSF models for this specific slice were generated. Reconstruction of other slices would require additional PSF models.

The PSF models of the forward projector were created by simulating ${ }^{99 \mathrm{~m}} \mathrm{Tc}$ point sources in GATE, positioned above the locations marked in Fig. 2 at distances from the detector ranging from 0 to 40 $\mathrm{cm}$ (in steps of $4.6 \mathrm{~mm}$ ). The simulations were made as realistic as possible by including the parallax effect, signal mixing, collimator attenuation and scatter, and septal penetration. The models were created for the flat panel detector as well as a conventional gamma camera (based on the Siemens Symbia T16 scanner). The intrinsic spatial resolution of the flat panel detector was assumed to be smaller than the detector pixel size. For the conventional gamma camera, a $3.8 \mathrm{~mm}$ full width at half maximum (FWHM) intrinsic spatial resolution was applied [12]. 
Table 1: Dimensions of the studied collimators.

\begin{tabular}{l|c|ccc} 
Collimator & Pixels per hole & Hole length $[\mathrm{mm}]$ & Hole width $[\mathrm{mm}]$ & Septal thickness [mm] \\
\hline LEHR & 4.12 & 24.05 & 1.110 & 0.160 \\
Custom LEHR & 4 & 24.05 & 1.077 & 0.155 \\
Enlarged holes & 8 & 24.05 & 2.154 & 0.310
\end{tabular}

The PSF models of the backward projector were created by positioning point sources of ${ }^{99 \mathrm{~m}} \mathrm{Tc}$ at the detector pixel locations and then tracing their backward path through the collimator. This results in a matrix with the potential source locations that can be included in the back projection step of the reconstructor.

\section{Collimator design}

In the design of a parallel-hole collimator, there exists a trade-off between sensitivity and resolution: by enlarging the collimator hole width, the detector sensitivity can be boosted at the expense of its resolution. Hence, in addition to the custom LEHR collimator (of 4-pixel hole width), a collimator with enlarged holes (with 8-pixel hole width) was also simulated. For every configuration, the septal thickness was set such that the septal penetration was $1 \%$ (see Tab. 1 for the obtained specific dimensions) [13].

\section{Reconstruction}

A simulation study was performed to study the reconstruction resolution and noise characteristics of the detector configurations. For every configuration, the detector simulated a body-tracing orbit with a $1 \mathrm{~cm}$ phantom-detector gap. Projections were created with 120 angles over 360 degrees and the reconstruction used the OSEM algorithm with 10 iterations and 8 subsets. The distributions were reconstructed on a 1496 x 1496 grid with $0.308 \mathrm{~mm}$ isotropic pixel size.

Photon attenuation was simulated using the accompanying attenuation maps. Photon scatter was challenging to implement since the reconstruction was performed in $2 \mathrm{D}$. To achieve a realistic scatter estimate, the $2 \mathrm{D}$ slice was during the reconstruction temporarily transformed to a downsampled symmetric 3D volume (136 x $136 \times 70$ grid with $3.388 \mathrm{~mm}$ isotropic voxel size) from which the scatter contribution to the $2 \mathrm{D}$ slice was calculated. The scattered photons were simulated using the Utrecht Monte Carlo System (UMCS) simulator [14,15]. This methodology is valid for the phantoms studied in this work since they have a attenuation map that is symmetric in height.

The reconstructions were made with or without detailed collimator modeling depending on the detector configuration. In the case of no detailed collimator modeling, only the depth dependence of the PSF models was taken into account (as is done in the majority of SPECT reconstructors). Similarly, the reconstructions were made with or without the capability for energy selection. In the case of energy selection, only the photopeak window (from 130 to $150 \mathrm{keV}$ ) was used. In the case of no energy selection, the entire energy spectrum was summed together. 
Four detector configurations were simulated. The reference reconstruction for comparison purposes was the Gamma camera (custom LEHR collimator, no detailed collimator modeling, with energy selection). This option illustrates the performance that can currently be achieved in the clinic. For the flat panel detector, the following three configurations were simulated:

- Flat panel detector - Standard (custom LEHR collimator, no detailed collimator modeling, no energy selection). This configuration illustrates the reconstruction performance if one uses the conventional reconstruction methodology for the currently used flat panel detector.

- Flat panel detector - With Modifications (enlarged collimator holes, with detailed collimator modeling, no energy selection). This configuration employs detailed collimator modeling to utilize the excellent intrinsic spatial resolution of the flat panel detector to obtain improved reconstruction resolution. The improved reconstruction resolution is hence traded with an improved detector sensitivity by enlarging the collimator holes in order to reduce the reconstruction noise.

- Flat panel detector - Future (enlarged collimator holes, with detailed collimator modeling, with energy selection). This configuration illustrates which reconstruction quality might be achieved for future flat panel detectors with the capability for photon energy selection.

Two distributions were simulated to assess the performance of the detector configurations:

- The Jaszczak phantom (consisting of a circular disk with cold rods of 4.8, 6.4, 7.9, 9.5, 11.1, and $12.7 \mathrm{~mm}$ in diameter) without Poisson noise. The average contrast of the $7.9 \mathrm{~mm}$ spheres was calculated as a measure of the reconstruction resolution.

- The NEMA IQ phantom (consisting of a background compartment with hot spheres of 10, $13,17,22,28$, and $37 \mathrm{~mm}$ in diameter added at an 8:1 ratio) with Poisson noise equivalent to $1000 \mathrm{MBq}$ total phantom activity at a $4.79 \mathrm{~mm}$ slice reconstruction with 30 seconds per projection measurement time. A post-reconstruction Gaussian filter of $5 \mathrm{~mm}$ FWHM was applied to smooth the reconstructions. Ten noise realizations were performed for every detector configuration to study the stability of the reconstructions. The maximum CNR of the $37 \mathrm{~mm}$ sphere was calculated (with the background compartment eroded by $5 \mathrm{~mm}$ ) as a measure of the reconstruction quality.

\section{Results}

\section{Proof-of-concept experiment}

The projection image obtained from the proof-of-concept experiment is illustrated in Fig. 3a. Since the acquisition time was long and the source activity was relatively high, an almost noiseless projection was obtained and the collimator structure can be easily observed in the image. The profile on the dashed line is shown together with the profile obtained from the simulation of the experiment in Fig. 3b. The agreement between the profiles indicates that the simulations are able to accurately model the physical measurements. 

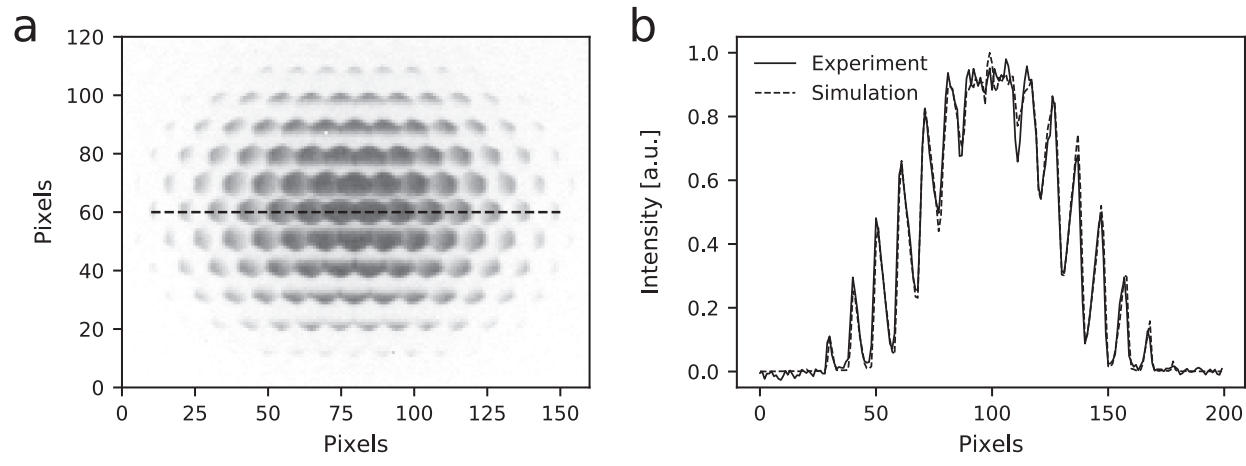

Figure 3: (a) Projection image obtained from the proof-of-concept experiment. (b) Line profile corresponding to the dashed line in the projection image, in arbitrary units (a.u.) together with the profile obtained from the simulation.

\section{Reconstruction}

\section{Gamma camera}

The reconstructions obtained from the conventional gamma camera (custom LEHR collimator, no detailed collimator modeling, with energy selection) are shown in Fig. 4a. The performance of this detector configuration can be currently achieved in the clinic and may be used as a reference to compare the quality of the flat panel reconstructions with.

\section{Flat panel detector - Standard}

The reconstructions obtained from the currently used flat panel detector when using conventional reconstruction technology (custom LEHR collimator, no detailed collimator modeling, no energy selection) are shown in Fig. 4b. The contrast in the Jaszczak phantom was lower than that achieved by the gamma camera due to scattered photons (since no photon energy selection was available). The CNR in the NEMA IQ phantom was lower than that of the gamma camera because of the scattered photons and the relatively lower detector sensitivity.

\section{Flat panel detector - With modifications}

The reconstructions obtained from the flat panel detector with modifications (enlarged collimator holes, with detailed collimator modeling, no energy selection) are shown in Fig. 4c. The contrast obtained in the Jaszczak phantom was similar to that achieved with the standard flat panel detector, which indicates that the detailed collimator modeling was able to accurately maintain the reconstruction contrast when moving to a lower-resolution collimator. The CNR in the NEMA IQ phantom improved when compared with the standard flat panel detector due to the relatively higher detector sensitivity.

\section{Flat panel detector - Future}

The reconstructions obtained from the future flat panel detector (enlarged collimator holes, with 

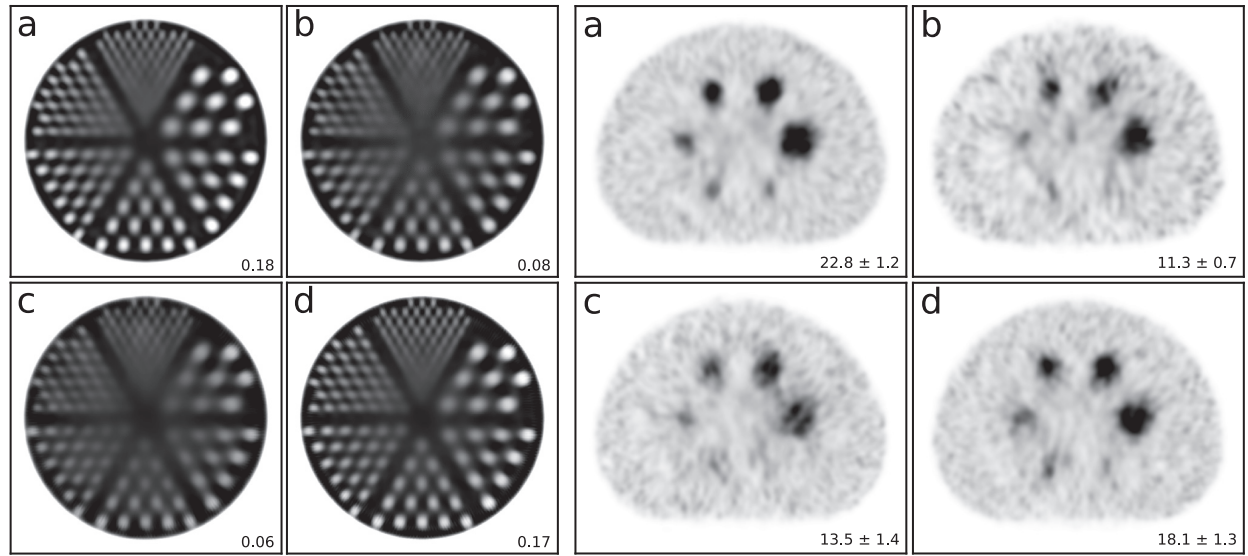

Figure 4: Reconstructions of the Jaszczak phantom and NEMA IQ phantom obtained from the (a) conventional gamma camera, (b) standard flat panel detector, (c) flat panel detector with modifications, and (d) future flat panel detector. For the Jaszczak phantom, the values reported in the bottom right represent the average contrast over the $7.9 \mathrm{~mm}$ rods. For the NEMA IQ phantom, they represent the maximum CNR of the $37 \mathrm{~mm}$ sphere averaged over the ten noise realizations.

detailed collimator modeling, with energy selection) are shown in Fig. 4d. By making use of the photon energy selection capability, most scattered photons could now be accurately discarded. The contrast in the Jaszczak phantom became similar to that obtained from the gamma camera. The CNR in the NEMA IQ phantom improved considerably over that achieved by the flat panel with modifications and approached the value obtained by the conventional gamma camera.

\section{Discussion}

Interventional procedures involving radionuclides would benefit from SPECT performed in the intervention room because the activity distribution could be immediately visualized. This study demonstrated the achievable SPECT reconstruction with the $\mathrm{x}$-ray flat panel of the C-arm CBCT scanner when using current technology, when making modifications to the collimator design and the reconstruction methodology, and when using projected future technology.

It is unknown which image quality will be required for interventional SPECT since such guided procedures have not yet been performed. However, some assumptions can be made based on current clinical care. For instance in radioembolization (which is of one the proposed applications for interventional SPECT scanning), it has been demonstrated that various quantitative measures (e.g. lung-shunting fraction, tumor uptake ratio) can be accurately determined down to $1 / 10^{\text {th }}$ of the clinically used number of counts [16-18]. These findings suggest that the flat panel detector (which similarly has a lower count rate) should also be able to retrieve accurate clinical results.

Unfortunately, it was not possible to perform SPECT with the proof-of-concept experiment because our approach required a pixel-matched collimator, which was not available. Therefore, this study was mainly intended as an initial exploration of the combined SPECT and CBCT scanner 
design. Using the results from this work as a reference, the next step would be to produce a pixelmatched collimator and to illustrate the feasibility of SPECT on a flat panel detector with physical phantom experiments.

The generation of the PSF models and the MLEM reconstructions are computationally very demanding. For these reasons, we chose to use employ a pixel-matched collimator and to only reconstruct in $2 \mathrm{D}$. There are no physical limitations in using other collimators or reconstructing in $3 \mathrm{D}$ except for the substantial increase in computational time. Variance reduction techniques and multiprocessing can improve the speed of both the generation of the PSF models and the MLEM reconstruction. We recommend using square-shaped pixel-matched collimators (as applied in CZT cameras) for future studies since this collimator design is symmetric in both directions and hence reduces the number of required PSF models.

The collimator needs to be designed very precisely for the detailed collimator modeling to function. Current lead collimators tend to possess small manufacturing errors, which makes their response unpredictable at times. There is, however, an increasing trend of manufacturing collimators via 3D printing. These printers can use more dense materials (e.g. tungsten) and can very precisely construct the collimator. We thus believe that collimator manufacturing will not be a limiting factor for the presented approach.

Similar to the above argument, the location of the collimator relative to the detector needs to be known with high precision. In the setting of interventional SPECT, the collimator would be placed on the CBCT scanner after the regular interventional procedure is finished. An x-ray shot could then be made to determine the collimator position relative to the flat panel detector.

The majority of CBCT scanners perform a circular orbit around the patient. For SPECT, however, it is desirable to perform a body-tracing orbit so that the best resolution is achieved. This would require some mechanical adjustments to the scanner design, but these are not expected to be limiting because most CBCT scanners already have large freedom in their movement. The extra weight introduced by the collimator might, however, require stronger motors for the detector rotation.

Summarizing the above points: although we have shown that it would be technically feasible to perform nuclear imaging with a flat panel detector, it is not expected that current CBCT scanners can be immediately used to perform interventional SPECT with. Most of the mentioned challenges should, however, be resolvable by either more computational power or small modifications to the CBCT scanner design. We thus believe it is interesting to further study the combined SPECT and $\mathrm{CBCT}$ scanner so that it might be used in future generation systems.

\section{Conclusion}

The x-ray flat panel detector of a C-arm CBCT scanner might be used to perform nuclear imaging. The SPECT reconstruction quality will be lower than that achieved by a conventional gamma camera. The flat panel detector approach could, however, be useful in providing a cost-effective alternative to the purchase of a mobile SPECT scanner for enabling interventional scanning. 


\section{References}

1. Van der Velden S, Kunnen B, Koppert WJC, Steenbergen JHL, Dietze MMA, Beijst C, Viergever MA, Lam MGEH, De Jong HWAM. A Dual-layer Detector for Simultaneous Fluoroscopic and Nuclear Imaging. Radiology 290(3):833-838 (2019)

2. Dietze MMA, Kunnen B, van der Velden S, Steenbergen JHL, Koppert WJC, Viergever MA, De Jong HWAM. Performance of a Dual-Layer Scanner for Hybrid SPECT/CBCT. Phys. Med. Biol. 64(10):105020 (2019)

3. Dietze MMA, Bastiaannet R, Kunnen B, van der Velden S, Lam MGEH, Viergever MA, De Jong HWAM. Respiratory Motion Compensation in Interventional Liver SPECT using Simultaneous Fluoroscopic and Nuclear Imaging. Med. Phys. 46(8):3496-3507 (2019)

4. Siewerdsen JH. Cone-beam CT with a flat-panel detector: From image science to image-guided surgery. Nucl. Instr. Meth. Phys. Res. Sect. A 648(S1):S241-S250 (2011)

5. Robert C, Montémont G, Rebuffel V, Buvat I, Guérin L, Verger L. Simulation-based evaluation and optimization of a new CdZnTe gamma-camera architecture (HiSens). Phys. Med. Biol. 55(9):2709-26 (2010)

6. Robert C, Montémont G, Rebuffel V, Verger L, Buvat I. Optimization of a parallel hole collimator/ CdZnTe gamma-camera architecture for scintimammography. Med. Phys. 38(4):1806-19 (2011)

7. Suzuki A, Takeuchi W, Ishitsu T, Tsuchiya K, Ueno Y, Kobashi K. A four-pixel matched collimator for high-sensitivity SPECT imaging. Phys. Med. Biol. 58(7):2199-217 (2013)

8. Suzuki A, Takeuchi W, Ishitsu T, Tsuchiya K, Morimoto Y, Ueno Y, Kobashi K, Kubo N, Shiga T, Tamaki N. High-sensitivity brain SPECT system using cadmium telluride (CdTe) semiconductor detector and 4-pixel matched collimator. Phys. Med. Biol. 58(21):7715-31 (2013)

9. Kasap S, et al. Amorphous and Polycrystalline Photoconductors for Direct Conversion Flat Panel X-Ray Image Sensors. Sensors 11(5):5122-57 (2011)

10. Seco J, Clasie B, Partridge M. Review on the characteristics of radiation detectors for dosimetry and imaging. Phys. Med. Biol. 59(20):R303 (2014)

11. Jan S, et al. GATE: A simulation toolkit for PET and SPECT. Phys. Med. Biol. 49(19):4543-61 (2004)

12. Siemens. Symbia $S$ and T System Specifications (2010)

13. Cherry SR, Sorenson JA, Phelps ME. Physics in Nuclear Medicine (2012)

14. De Jong HWAM, Slijpen ETP, Beekman FJ. Acceleration of Monte Carlo SPECT simulation using convolution-based forced detection. IEEE Trans. Nucl. Sci. 48(1):58-64 (2001)

15. Dietze MMA, Van der Velden S, Lam MGEH, Viergever MA, De Jong HWAM. Fast quantitative reconstruction with focusing collimators for liver SPECT. EJNMMI Phys. 5(1):28 (2018)

16. Van der Velden S, Dietze MMA, Viergever MA, De Jong HWAM. Fast technetium-99m liver SPECT for evaluation of the pretreatment procedure for radioembolization dosimetry. Med. Phys. 46(1):345355 (2019)

17. Dietze MMA, Kunnen B, Beijst C, De Jong HWAM. Adaptive Scan Duration in SPECT: Evaluation for Radioembolization. Med. Phys. 47(5):2128-2138 (2020)

18. Kunnen B, Dietze MMA, Braat AJAT, Lam MGEH, Viergever MA, De Jong HWAM. Feasibility of imaging ${ }^{90} \mathrm{Y}$ microspheres at diagnostic activity levels for hepatic radioembolization treatment planning. Med. Phys. 47(3):1105-1114(2020) 


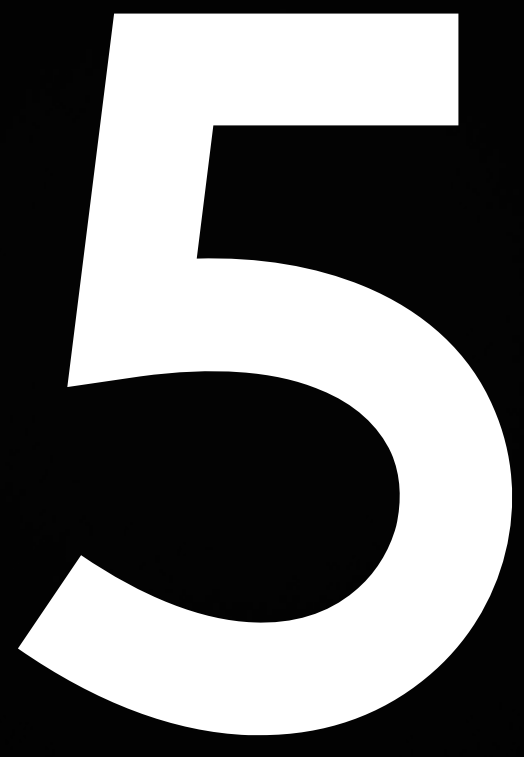




\section{RESPIRATORY MOTION COMPENSATION FOR INTERVENTIONAL IMAGING}

PUBLISHED AS:

MMA Dietze, R Bastiaannet, B Kunnen, S van der Velden, MGEH Lam, MA Viergever, HWAM de Jong Respiratory Motion Compensation in Interventional Liver SPECT using Simultaneous Fluoroscopic and Nuclear Imaging 


\section{Abstract}

\section{Purpose}

Quantitative accuracy of the SPECT reconstruction of the pre-treatment procedure of liver radioembolization is crucial for dosimetry; visual quality is important for detecting doses deposited outside the planned treatment volume. Quantitative accuracy is limited by respiratory motion. Conventional gating eliminates motion by count rejection but increases noise, which degrades the visual reconstruction quality. Motion compensation using all counts can be performed if the motion signal and motion vector field over time are known. The measurement of the motion signal of a patient currently requires a device (such as a respiratory belt) attached to the patient, which complicates the acquisition. The motion vector field is generally extracted from a previously acquired $4 \mathrm{D}$ scan and can differ from the motion in the scan performed during the intervention. The simultaneous acquisition of fluoroscopic and nuclear projections can be used to obtain both the motion vector field and the projections of the corresponding (moving) activity distribution. This eliminates the need for devices attached to the patient and provides an accurate motion vector field for the SPECT reconstruction. Our approach to motion compensation would primarily be beneficial for interventional SPECT because the time-critical setting requires fast scans and no inconvenience of an external apparatus. The purpose of this work is to evaluate the performance of the motion compensation approach for interventional liver SPECT by means of simulations.

\section{Methods}

Nuclear and fluoroscopic projections of a realistic digital human phantom with respiratory motion were generated using fast Monte Carlo simulators. Fluoroscopic projections were sampled at 1-5 Hz. Nuclear data were acquired continuously in list mode. The motion signal was extracted from the fluoroscopic projections by calculating the center-of-mass, which was then used to assign each photon to a corresponding motion bin. The fluoroscopic projections were reconstructed per bin and coregistered, resulting in a motion vector field that was used in the SPECT reconstruction. The influence of breathing patterns, fluoroscopic imaging dose, sampling rate, number of bins, and scanning time was studied. In addition, the motion compensation method was compared with conventional gating to evaluate the detectability of spheres with varying uptake ratios.

\section{Results}

The liver motion signal was accurately extracted from the fluoroscopic projections, provided the motion was stable in amplitude and the sampling rate was greater than $2 \mathrm{~Hz}$. The minimum total fluoroscopic dose for the proposed method to function in a five-minute scan was $10 \mu \mathrm{Gy}$. Although conventional gating improved the quantitative reconstruction accuracy, substantial background noise was observed in the short scans because of the limited counts available. The proposed method similarly improved the quantitative accuracy, but generated reconstructions with higher visual quality. The proposed method provided better visualization of low-contrast features than when using gating. 


\section{Conclusion}

The proposed motion compensation method has the potential to improve SPECT reconstruction quality. The method eliminates the need for external devices to measure the motion signal and generates an accurate motion vector field for reconstruction. A minimal increase in the fluoroscopic dose is required to substantially improve the results, paving the way for clinical use. 


\section{Introduction}

Respiratory motion is a major degrading factor for the quantitative accuracy of single photon emission computed tomography (SPECT) scans and should ideally be compensated for in the reconstruction [1-3]. Clinically, the effect of motion is mitigated by applying gating: a projection set is generated by only incorporating data with a minimum motion amplitude [4]. This improves the resolution but increases the noise owing to the limited counts that are accepted, especially when performing fast scans. More elaborate methods that use all data additionally require a motion vector field: a map of the patient-specific organ movements and deformations over the respiratory cycle [5]. Using such a model, the data from all individual motion phases can be combined into a single motion-corrected reconstruction [6].

To extract the motion signal, data-driven approaches are often used in other modalities $[7,8]$. However, since SPECT scans are often limited by count statistics, data-driven approaches are more challenging for this modality [9] and additional information is usually required. For instance, the respiratory signal can be tracked using a respiratory belt, which measures the expansion of the abdomen over time. Other frequently used approaches include camera systems and spirometers [10]. Most of these devices require extra work for the technicians and are difficult to work with in time-critical situations.

It is difficult to construct a general model for the motion vector field due to inter-patient variability and different breathing preferences [11]. Therefore, the most common approach to estimate the motion vector field is to extract it per patient from a previously acquired $4 \mathrm{D}$ scan, such as CT [12] or magnetic resonance imaging (MRI) [13]. However, observed motion can depend appreciably on the scan modality or procedure [14], e.g., because of different levels of stress or scan duration. Hence, the motion vector field from these scans potentially describes the motion in the scan of interest suboptimally.

A device that simultaneously measures nuclear and fluoroscopic projections [15,16], and that is now under construction, could be used to intrinsically correct for respiratory motion. The motion signal can be extracted from the fluoroscopic projections and be used to allocate the nuclear and fluoroscopic projections to motion bins. The fluoroscopic projections are reconstructed per bin and then registered to each other to extract the motion vector field over time, which can subsequently be included in the SPECT reconstruction [17]. A single, averaged motion vector field is thus used to correct the data over the entire scan. The advantages of such a device are twofold: no external devices are required to extract the motion signal, simplifying the procedure in the clinic, and the motion vector field is retrieved from the same scan, ensuring that changes in breathing between several scans do not influence motion compensation performance. The disadvantages are that gamma sensitivity is reduced and extra dose is administered.

Our approach to motion compensation will most likely not be suitable for general motion compensation, but there are situations in which it could be of benefit. The proposed use for our device is in hepatic radioembolization, in which small radioactive microspheres are inserted into the liver [18]. A pre-treatment SPECT/CT scan of technetium-99m macroaggregated albumin 


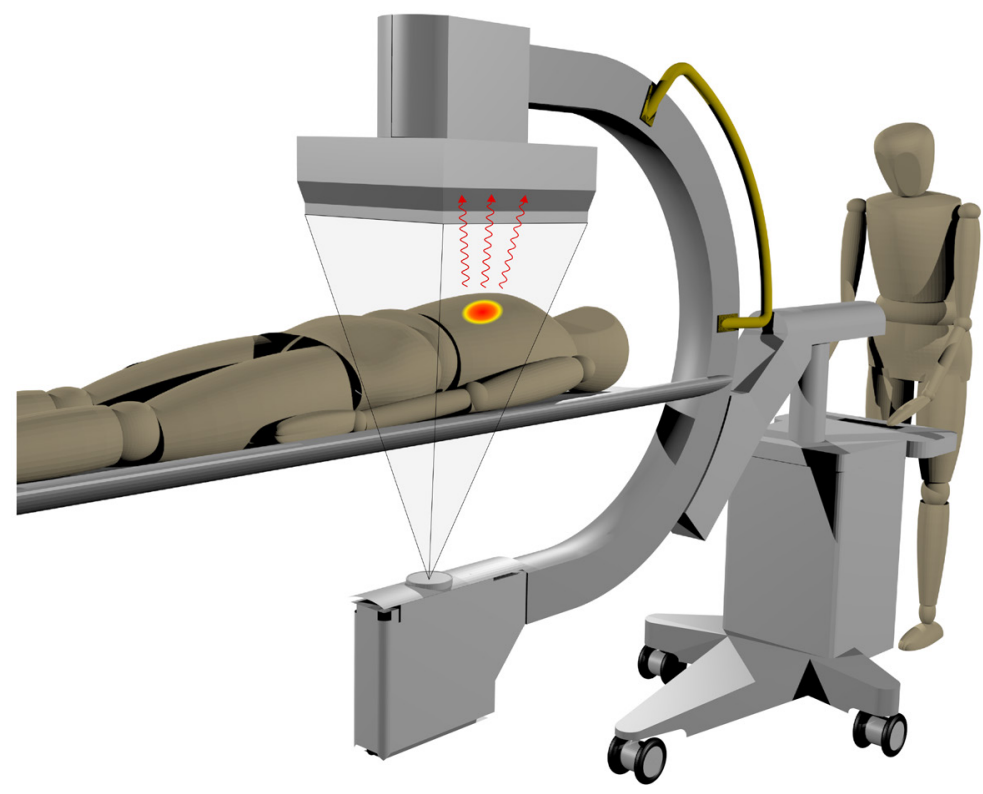

Figure 1: The proposed detector that can measure fluoroscopic and nuclear projections simultaneously. The depicted cone is the irradiated $\mathrm{x}$-ray area that is captured on the flat panel. In red are the gamma photons that pass through the flat panel to be detected on the gamma camera.

is normally performed before therapy to detect potential inadvertent regions of high activity and for the treatment planning of dosimetry. Ideally, this pre-treatment procedure and therapy are performed in a single setting (a one-day procedure) to minimize changes in anatomy and the catheter position [19]. Our mobile dual-layer detector would be moved into the intervention room after the pre-treatment procedure to perform an interventional SPECT scan. The activity distribution would be assessed, after which the physician can start the therapy. Our proposed approach to motion compensation is expected to be beneficial for this situation because the time-critical setting requires fast scans and no inconvenience of an external apparatus.

The purpose of this work is to evaluate the quantitative accuracy and visual quality retrieved from the proposed motion compensation approach for fast interventional liver SPECT in a simulation study. The influence of the fluoroscopic imaging dose, sampling rate, total scanning time, number of bins, and motion patterns will be studied. We will investigate whether inadvertent regions of high activity could be better distinguished with our motion compensation technique than with gating.

\section{Methods}

\section{Detector}

An overview of the proposed detector system is shown in Fig. 1. The system consists of a gamma camera with a $100 \mathrm{~cm}$ focal length low-energy, high-resolution (LEHR) cone beam collimator, 


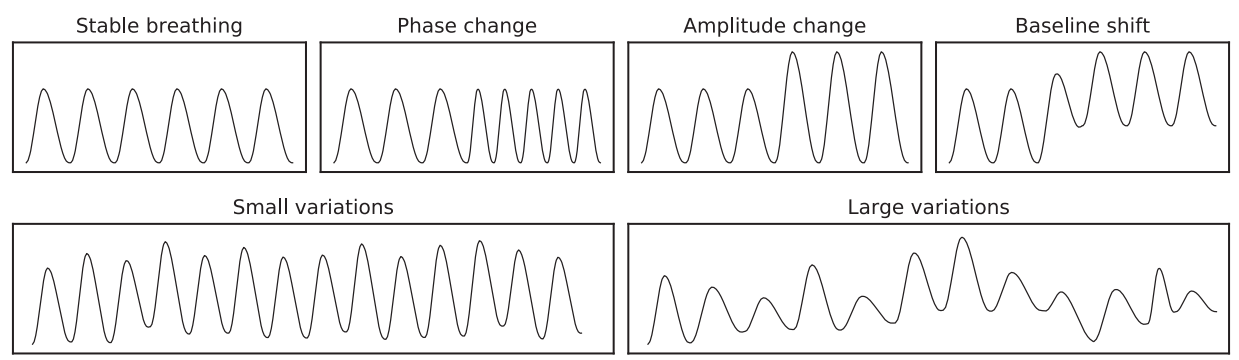

Figure 2: The respiratory motion patterns used in this study: stable breathing and five types of irregular breathing. Visualized is the diaphragm amplitude over time.

merged with a cone beam CT (CBCT) flat panel in front of it. This dual-layer detector is, together with the $\mathrm{x}$-ray tube, mounted on a mobile c-arm so it can be used during interventions. Most generated $\mathrm{x}$-rays will be absorbed in the flat panel; the gamma photons possess a higher energy, allowing a major fraction to pass through the flat panel to be detected on the gamma camera. The custom flat panel anti-scatter grid was not included because the CBCT reconstruction quality is not of key importance. A prototype system has shown the feasibility of this configuration $[15,16]$.

\section{Phantom}

A realistic patient phantom was generated using the XCAT phantom program [20] with the standard body settings of a $95 \mathrm{~kg}$ male. Respiratory motion was generated with 50 samples per breathing cycle, for one stable and five irregular breathing patterns (see Fig. 2) [21]. The six studied patterns were:

- Stable breathing: respiratory cycle of five seconds with a maximum diaphragm amplitude of $2.0 \mathrm{~cm}$ and anterior-posterior (AP) expansion of $1.2 \mathrm{~cm}$

- Phase change: patient switches halfway in the procedure from stable breathing to a respiratory cycle of three seconds

- Amplitude change: patient switches halfway from stable breathing to a maximum diaphragm amplitude of $3.0 \mathrm{~cm}$ and AP expansion of $1.8 \mathrm{~cm}$

- Baseline shift: patient switches halfway from stable breathing to a baseline $1.0 \mathrm{~cm}$ higher than the previous baseline

- Small variations: a motion signal with a respiratory cycle of five seconds is generated by randomly assigning a maximum amplitude (between 2.0 and $2.5 \mathrm{~cm}$; scaled AP expansion) and baseline shift (between 0.0 and $0.5 \mathrm{~cm}$ ).

- Large variations: a motion signal is generated by randomly assigning a maximum amplitude (between 0.5 and $2.0 \mathrm{~cm}$; scaled AP expansion), phase (between three and seven seconds), and baseline (between 0.0 and $1.0 \mathrm{~cm}$ ). 

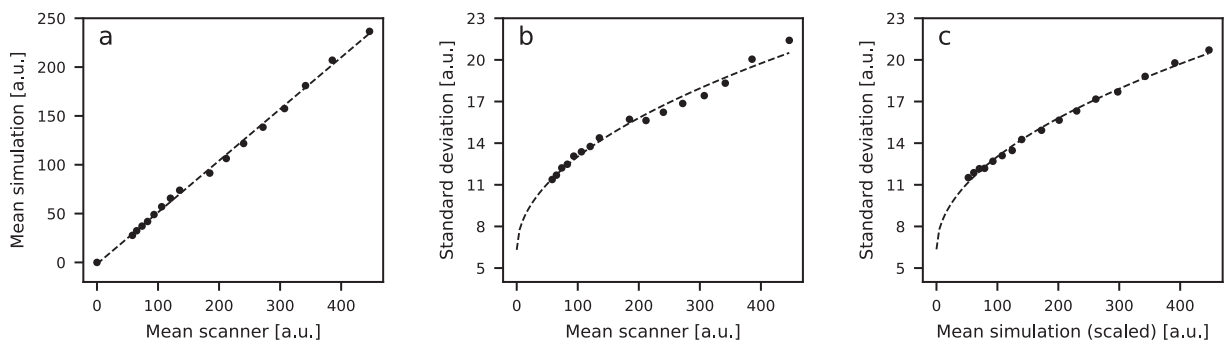

Figure 3: Results on the dose-noise relation in arbitrary units (a.u.): (a) shows the relation between the mean value in the tiles of varying copper thickness observed in the simulation and the scanner projections; (b) shows the standard deviation of the scanner projections as a function of the mean value; and (c) shows that similar noise can be generated on the simulated projections by incorporating the parameters of the scanner fit. The mean values of the simulation were scaled to the mean values of the scanner.

A total of $150 \mathrm{MBq}{ }^{99 \mathrm{~m}} \mathrm{Tc}$ was inserted into the liver, in which a sphere of $30 \mathrm{~mm}$ in diameter was added. Several uptake ratios $(2: 1,3: 1,4: 1$, and 5:1) were evaluated to study the detectability for the different imaging protocols. Activity and attenuation maps were generated on a $128 \times 128 \times 100$ matrix with $4.7 \mathrm{~mm}$ isotropic voxels.

\section{Projection generation}

Fluoroscopic projections were generated in GATE [22], using the fixed forced detection variance reduction actor for the scatter generation [23]. Scatter was simulated on a factor 4 down-scaled attenuation map, using $5 \times 10^{5}$ particle instances per projection. The input beam spectrum was retrieved from the Simulation of Fluoroscopic Spectra tool [24] at the clinically used setting of $120 \mathrm{kVp}$ with a $1.0 \mathrm{~mm}$ copper filter. The CBCT flat panel was positioned $27 \mathrm{~cm}$ from the body center. The gamma transmission through the flat panel was set at $64 \%$, in line with what we expect to achieve at $140 \mathrm{keV}$ [15].

Nuclear projections were generated using the Utrecht Monte-Carlo System (UMCS), which is a fast Monte Carlo-based software package that simulates particle interactions in the body [25]. The forward projector has previously been validated for several isotopes [26-28] and has been extended to converging collimators [29]. An energy window of $15 \%$ was set around the $140 \mathrm{keV}$ ${ }^{99} \mathrm{~m} T \mathrm{Tc}$ photopeak and the point-spread function was specifically generated for the proposed detector system, making quantitative reconstructions possible. The intrinsic spatial resolution of the gamma camera was set at a $3.8 \mathrm{~mm}$ full width at half maximum [30]. The flat panel thickness $(2 \mathrm{~cm})$ increased the nuclear orbit radius to $29 \mathrm{~cm}$. Poisson noise, scaled with the time per projection and the total activity, was added to the projections.

Both fluoroscopic and nuclear projections were generated for 120 angles over 360 degrees for the different breathing patterns at a camera size of $40 \times 50 \mathrm{~cm}^{2}$. Two protocols were investigated: one with a scan time of 5 minutes, which will be referred to as the fast interventional protocol, and one with a scan time of 30 minutes, which is current clinical practice. The fluoroscopic sampling rate was varied from $1-5 \mathrm{~Hz}$ and the total fluoroscopic dose from 1 to $1000 \mu \mathrm{Gy}$. Additionally, we 

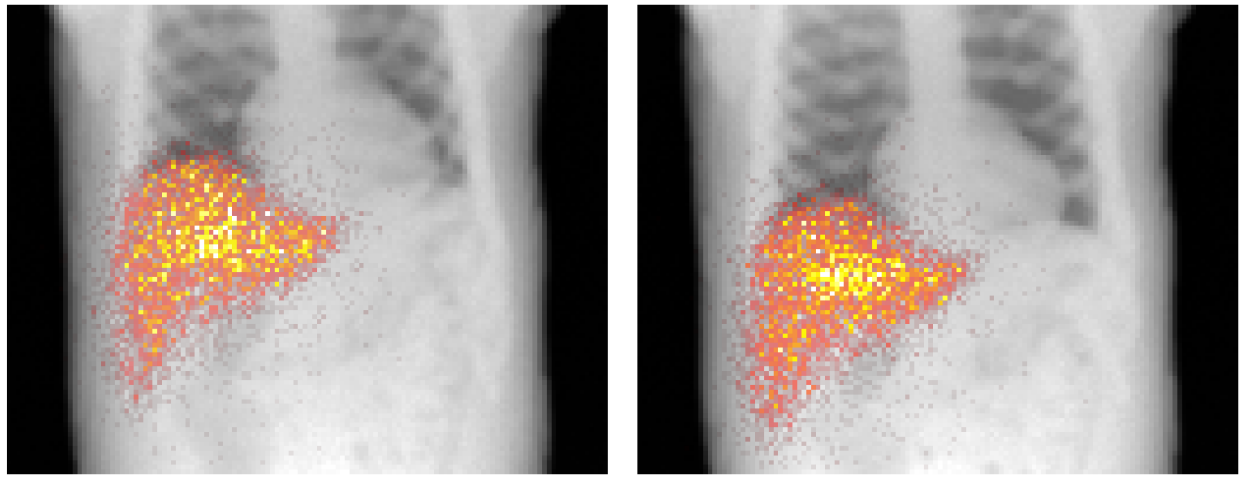

Figure 4: Fluoroscopic (gray scale) and nuclear (color) projections for two respiratory phases (left: amplitude of $0 \mathrm{~cm} /$ right: $5 \mathrm{~cm}$ ).

investigated whether it would be better to increase the sampling rate or increase the fluoroscopic dose per view, while keeping the total fluoroscopic dose level constant.

\section{Projection noise}

To realistically simulate the effect of the fluoroscopic dose on the reconstruction results, a dosenoise relation for the fluoroscopic projections is required. To this end, the noise characteristics of a clinical CBCT scanner (Allura FD20; Philips Healthcare, Amsterdam, The Netherlands) were studied. An image quality phantom (Fluorad A+D; Pehamed, Sulzbach, Germany), consisting of 16 tiles of varying copper thicknesses (representing the range of attenuation in patients), was scanned using a clinical high-dose CBCT protocol, while monitoring the air kerma reported on the scanner. Additionally, the phantom configuration (as reported in the manual) was simulated in GATE.

The mean values and standard deviations were measured in a square of $74 \mathrm{x} 74$ pixels positioned on the center of the tiles for a single projection. The fluoroscopic detector noise consists of both a Gaussian (electrical) component and a Poisson distributed component [31]. The Poisson noise is expected to scale with the square root of the number of detected particles, while the Gaussian noise depends only on the size of the detector pixels. The observed standard deviation for the tiles was therefore fitted with:

$$
\sigma=\sigma_{0}+c \sqrt{\mu}
$$

where $\sigma$ is the standard deviation on the tile, $\sigma_{0}$ the electrical noise, c a constant, and $\mu$ the mean value. A change in pixel size results in the scaling of the electrical noise $\sigma_{0}$ with the square root of the resize factor and in a linear scaling of the mean value $\mu$.

Using the relation between the mean values of the experimental and simulated (noise-free) projections and the reported air kerma, the noise in the simulations can be generated as a function of the fluoroscopic dose. In practice, this fluoroscopic dose could be tuned by changing the exposure 

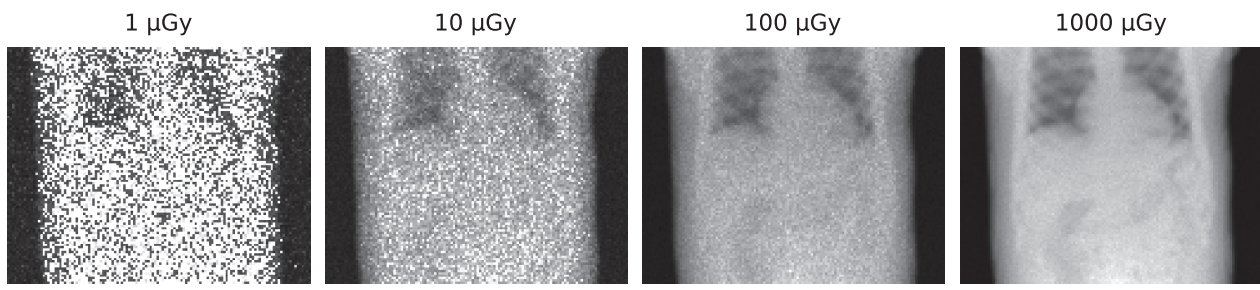

Figure 5: Effect of fluoroscopic dose on the fluoroscopic projections. Reported is the total fluoroscopic dose (air kerma) for all projections in a five-minute scan while sampling at $5 \mathrm{~Hz}$.

(mA per projection). Therefore, the fluoroscopic dose would scale linearly with the number of particles detected, thus only influencing the Poisson noise component.

\section{Motion signal extraction}

The motion signal was extracted from the pixel intensity distribution of the simulated fluoroscopic projections. Over a vertical strip of 50 pixels, centered on the lung-liver boundary, the center-ofmass was calculated for every sampled fluoroscopic projection. The collection of centers-of-masses over the projection angles has a background component, which was removed by subtracting the time-averaged signal. Peaks found in the remaining signal were used to normalize all values between no motion and the maximum motion amplitude. All projections were then binned by placing the bin boundaries at equal distances based on the motion amplitude.

If the number of bins is increased, the resolution of the reconstruction should improve, as more accurate compensation for motion is made. However, the computational time scales linearly with the number of bins and hence an optimum should be found.

\section{CBCT reconstruction}

The fluoroscopic projection sets were reconstructed per bin using TIGRE [32], which is a fast GPU-based CBCT reconstruction package with several options for iterative algorithms. The reconstruction was performed using the ordered-subset simultaneous algebraic reconstruction technique (OS-SART) with 50 iterations. This iterative reconstruction has an advantage over the regular clinical FDK algorithm [33] because it suppresses noise.

\section{Registration}

The CBCT reconstruction of the first bin was registered to the reconstructions of the other bins using the Elastix image registration package [34]. The deformable registration provided a motion vector field from the first bin to the other bins. The inverse motion vector fields were retrieved by performing registration with the fixed and moving volumes exchanged, i.e., the registration of the CBCT reconstructions of the other bins to that of the first bin. The vector fields were mean filtered over three voxels to increase the smoothness and mean filtered over three motion bins to ensure temporal regularity. 


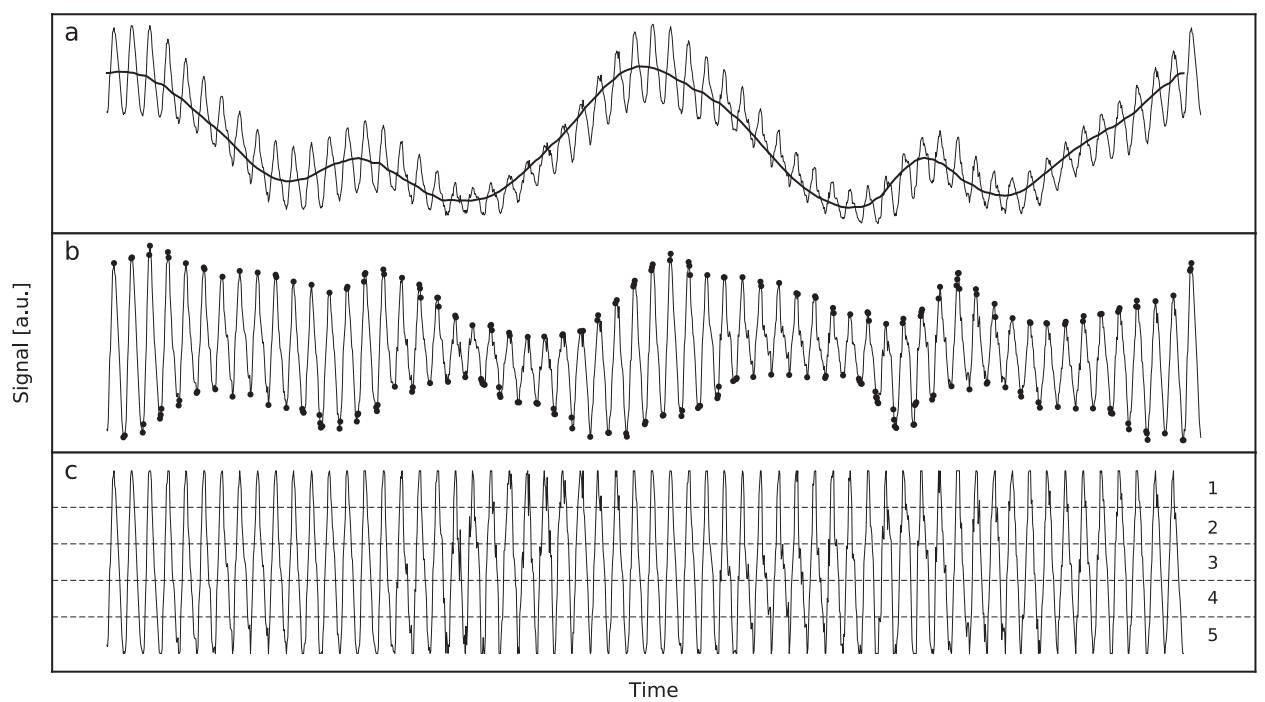

Figure 6: Extraction of the motion signal (acquired with stable motion, a five-minute scan, a $5 \mathrm{~Hz}$ sampling rate, and a $1000 \mu \mathrm{Gy}$ fluoroscopic dose) from the fluoroscopic projections using a center-of-mass approach. Frame (a) is the raw motion signal with the time-averaged signal; (b) the signal with background removed, with the found peaks; and (c) the normalized signal with five bin boundaries.

The quality of the CBCT reconstruction is expected to be low (due to a relatively high scatter contribution, small motion artifacts, potential partial sampling, and high noise in the projections). The liver contours themselves may therefore not be tracked over time. Instead, several regions were selected from which to extrapolate the motion. For the superior-inferior direction, the vector field below the lungs was set to the motion observed in the diaphragm. For the anterior-posterior direction, the vector field below the lungs was set to the motion observed in the sternum. The spine was assumed to be fixed.

\section{SPECT reconstruction}

The nuclear projections were reconstructed using UMCS, with the same settings as in the projection generation. No subsets were used, as the number of projections was not constant for all options. All reconstructions were performed with 25 iterations and 10 noise realizations were made to study the stability of the metrics to noise. Only the dual-layer configuration was studied in this work, which means that the flat panel was present for all simulated options.

The following options for SPECT reconstruction were compared:

- No motion compensation. The nuclear projections were reconstructed with no form of motion compensation. This is the current practice in most hospitals.

- Use of gating. The estimated motion signal was used to select the projections in the breathing cycle with minimum motion: $1 / 5^{\text {th }}$ of the motion amplitude was used. 

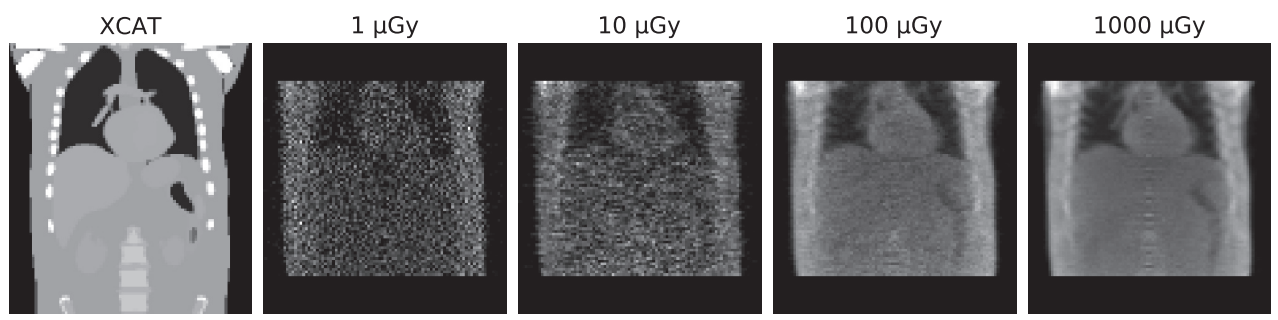

Figure 7: Reconstructions of one bin for the studied fluoroscopic dose levels (acquired with stable motion, a five-minute scan, and a $5 \mathrm{~Hz}$ sampling rate), together with the XCAT phantom used as input for the fluoroscopic projections.

- Use of the motion vector field. For every iteration during reconstruction and for every bin, the intermediate motion-free SPECT reconstruction was translated to each respective motion bin using the associated motion vector field. The estimated projections were obtained from the forward projection of the translated reconstruction image, and the error projections were calculated by dividing the estimated projections by the true projections (that should have the same translation due to motion). The error image was obtained by performing backprojection of the error projections. The error images of all bins were translated back to the stationary motion bin, and these images were averaged. This final error image was used to update the intermediate motion-free SPECT reconstruction, after which the next iteration was started.

- No motion. A projection set with no motion was generated and reconstructed. These reconstructions were used as a reference.

To correct for photon attenuation, an attenuation map is required. We assumed that a breathhold diagnostic CT was available. This high-quality CT would be registered to the obtained lowquality CBCT and scaled to generate the breath-hold attenuation map. For the motion vector field reconstruction option, a $4 \mathrm{D}$ attenuation set was subsequently made by applying the respiratory motion transformations.

The incorporation of motion into the SPECT reconstruction was evaluated with two vector fields: one estimated from the motion vector field during the procedure with the proposed detector system, and one that could be obtained from a previously acquired 4D scan. Since motion will vary between scans, the latter vector field was assumed to underestimate the true respiratory motion by $2 \mathrm{~mm}$ for the maximum diaphragm motion (resulting in $2.0-0.2=1.8 \mathrm{~cm}$ max motion) and $1 \mathrm{~mm}$ for the AP expansion (resulting in $1.2-0.1=1.1 \mathrm{~cm}$ max motion) [35].

The reconstruction results were evaluated in terms of the activity recovery (as a measure of quantitative accuracy) and contrast-to-noise ratio (CNR) (as a measure of visual quality). The CNR has the additional benefit that it reaches a maximum value, which eliminates the effect of different convergence rates for the several reconstruction methods. Practically, this means that a certain iteration number does not have to be chosen. 

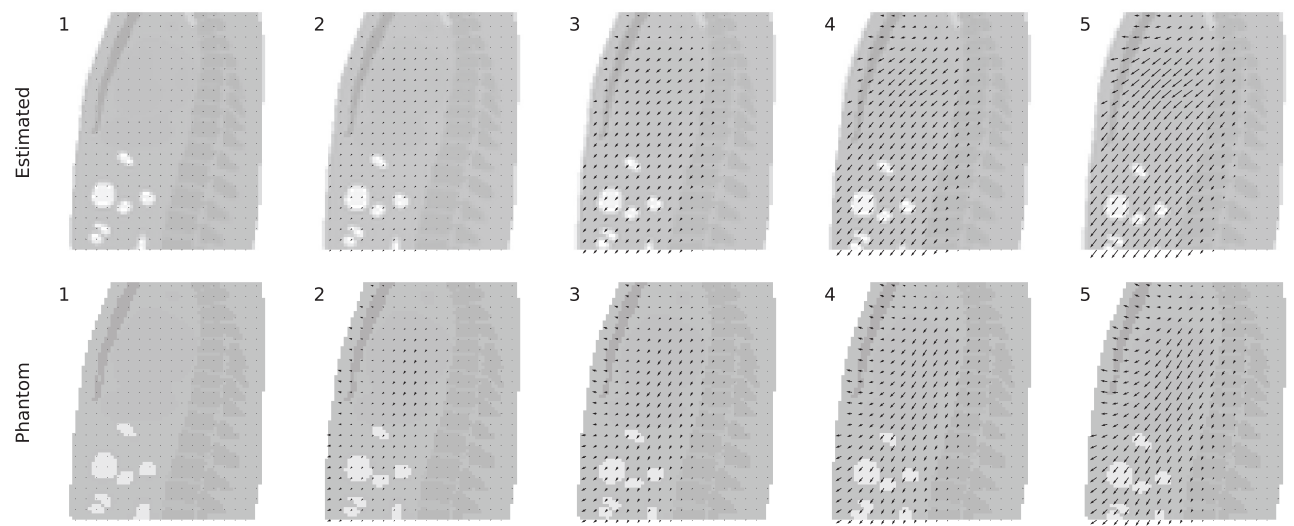

Figure 8: Estimated motion vector field for five bins (acquired with stable motion, a five-minute scan, a $5 \mathrm{~Hz}$ sampling rate, and a $1000 \mu \mathrm{Gy}$ fluoroscopic dose) with the transformed attenuation map (top) and the phantom motion field with the phantom attenuation map (bottom).

\section{Results}

\section{Fluoroscopic dose-noise relation}

The relation between the mean projection values of the tiles as measured by the clinical CBCT scanner and those simulated with GATE is shown in Fig. 3a. The relation was found to be linear, which indicates that the simulation is an accurate tool for predicting the scanner projections. Fig. $3 \mathrm{~b}$ shows the relation between the standard deviation and the mean values in the scanner projections. Since the curve crosses the $\mathrm{x}$-origin at a value larger than zero, the electrical noise is important in describing the scanner noise.

With the combined information of the relation between the scanner mean and simulation mean, and the scanner standard deviation, the noise was generated in the simulations. The results are presented in Fig. 3c, which shows a similar noise pattern as in the scanner projections. This noise is now directly linked to fluoroscopic dose and will be used for further analyses.

\section{Projection generation}

Two nuclear projections ( $0.5 \mathrm{sec}$ in duration) and corresponding fluoroscopic projections (total fluoroscopic dose level of $1000 \mu \mathrm{Gy}$ ) at a diaphragm motion of 0 and $5 \mathrm{~cm}$ for the phantom with a 5:1 uptake ratio are shown in Fig. 4 for illustration purposes. Histogram equalization was performed on the shown fluoroscopic projections to increase the soft-tissue contrast for visualization.

The effect of noise and scatter on the fluoroscopic images can be studied in more detail by evaluating the fluoroscopic projections for the different fluoroscopic dose levels in Fig. 5. It becomes increasingly difficult to identify the individual organs at a lower fluoroscopic dose, but the lung-liver boundary can be visualized even for very low dose levels. 


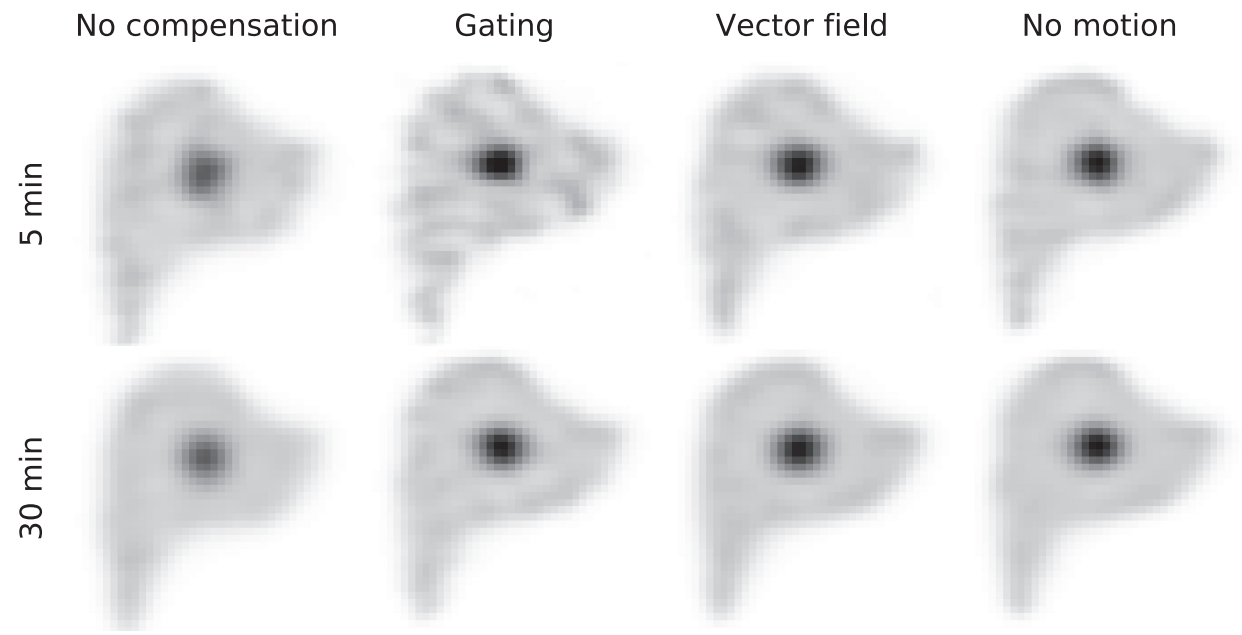

Figure 9: Reconstructions of the nuclear liver images for stable motion for a single noise realization, with both the five- and 30-minute scans for the phantom with a 5:1 uptake ratio. Reconstruction was performed without motion compensation, with gating, and with vector field compensation (acquired with a $5 \mathrm{~Hz}$ sampling rate and a 1000 or $6000 \mu \mathrm{Gy}$ fluoroscopic dose). As a reference, the last column shows the reconstructions for the case in which no motion was present. The iteration in which the maximum CNR was obtained is shown.

\section{Motion signal extraction}

Fig. 6 shows the motion signal that was extracted from the fluoroscopic projections for a stable respiratory pattern (acquired with a $1000 \mu \mathrm{Gy}$ fluoroscopic dose, a $5 \mathrm{~Hz}$ sampling rate, and a fiveminute scan). Fig. 6 a shows the raw signal obtained from the center-of-mass tracking, together with the time-averaged signal. Fig. $6 \mathrm{~b}$ shows the signal with the background component removed (i.e., the raw signal minus the time average), together with the found peaks. Fig. $6 \mathrm{c}$ shows the signal normalized to the peaks, together with the bin boundaries.

\section{CBCT reconstruction}

The binned fluoroscopic projections were reconstructed per bin, and the result for one bin is shown in Fig. 7. Some artifacts towards the ribs are present due to intra-bin motion. The CBCT reconstruction image contrast was lower than that of the XCAT phantom due to beam hardening and the contribution of scattered photons. In the upper and lower parts of the reconstruction, some volume is truncated because of the scanner size and the cone geometry. Again, it is difficult to localize the individual organs, but the lung-liver barrier can be well identified.

\section{Motion vector field}

The CBCT reconstructions of the binned fluoroscopic projections were coregistered, from which the motion field was extracted. Fig. 8 shows the evolution of the motion field over five bins, together with the motion field of the phantom. The motion in both the superior-inferior and anteriorposterior directions is evident. The abdomen is enlarged in size in the transformed attenuation map over the respiratory cycle. 


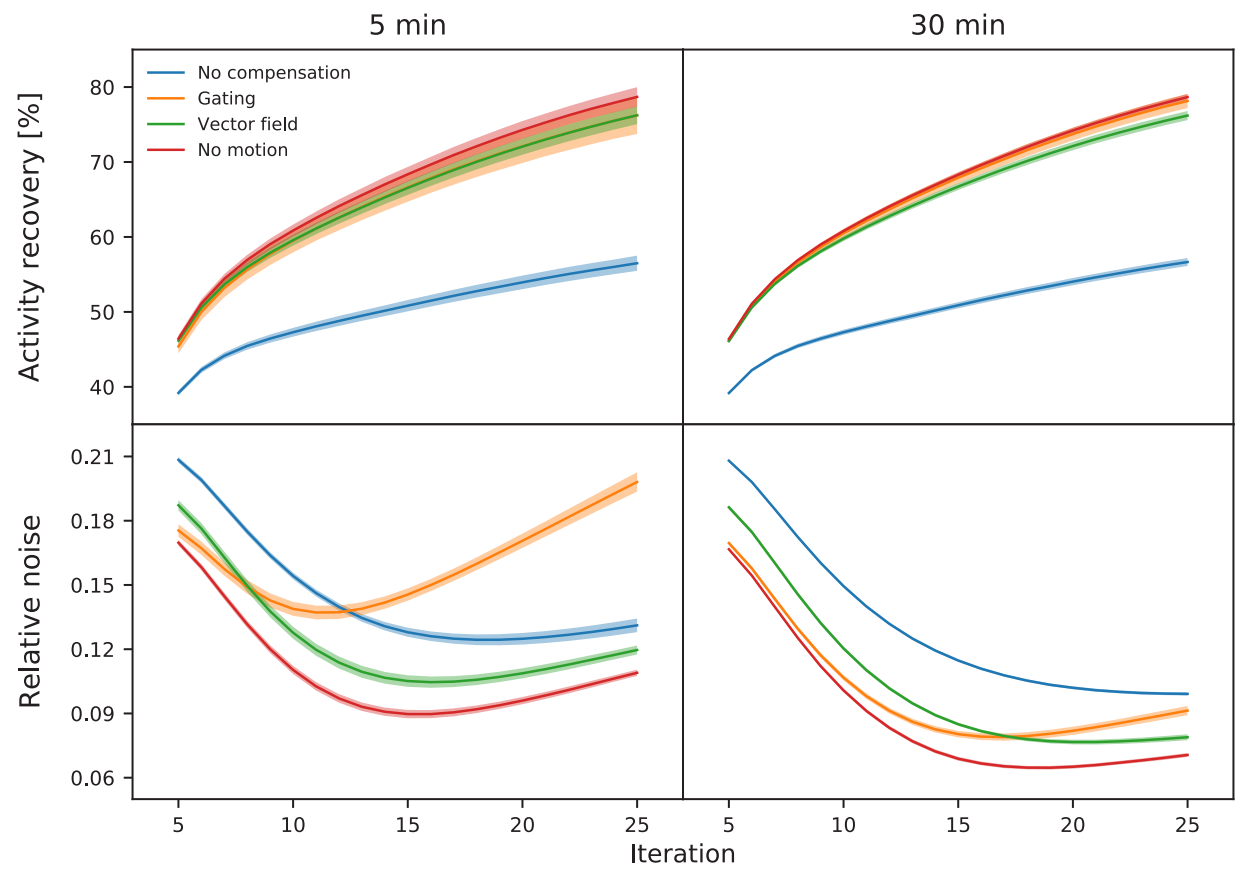

Figure 10: Sphere activity recovery and relative background noise for the motion compensation options, shown for the five- and 30-minute scans (acquired with stable motion, a $5 \mathrm{~Hz}$ sampling rate, and a 1000 or $6000 \mu \mathrm{Gy}$ fluoroscopic dose) for the phantom with a 5:1 uptake ratio as a function of the iteration number. The shaded regions represent the standard deviation obtained from the noise realizations.

\section{SPECT reconstruction}

Fig. 9 shows the SPECT reconstructions for the four reconstruction methods for both the five- and 30-minute scans (acquired with stable motion, a $5 \mathrm{~Hz}$ sampling rate, five bins, and a $1000 \mu \mathrm{Gy}$ (five-minute) or $6000 \mu \mathrm{Gy}$ (30-minute) fluoroscopic dose). Visual inspection shows that no motion compensation resulted in a low contrast between the sphere and liver background. Gating resulted in a high-contrast image, but the noise in the background was increased, which was especially evident in the five-minute scan. Motion compensation resulted in a high sphere contrast and low noise levels. This option best resembled the reconstructions with no motion present.

The above results are presented in more detail in Fig. 10, which shows the activity recovery and the relative noise levels (standard deviation divided by the average intensity) as a function of the iteration number. These results confirm that both gating and the vector motion field compensation provided good quantitative accuracy, but noise quickly dominated in the case of gating.

Using the contrast-to-noise ratio, the above results can be combined in a single measure. The maximum CNR over the iterations was determined and is shown in Fig. 11. This figure shows how gating performed relatively better for the 30-minute scan than for the five-minute scan, thanks to the increased number of counts available for reconstruction. 

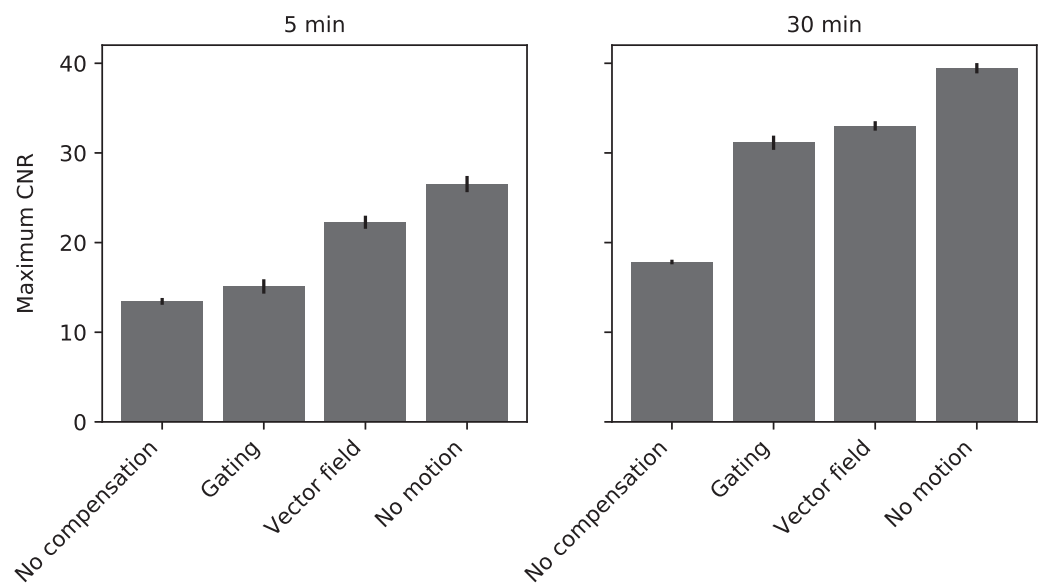

Figure 11: Maximum CNR of the sphere in the SPECT reconstruction for the various reconstruction methods, for the five- and 30-minute scans (acquired with stable motion, a $5 \mathrm{~Hz}$ sampling rate, and a 1000 or $6000 \mu \mathrm{Gy}$ fluoroscopic dose) for the phantom with a 5:1 uptake ratio.

\section{Reconstruction properties}

The maximum CNR for the studied reconstruction properties for the five-minute scan are shown in Tab. 1. The standard vector field compensation is the reconstruction performed with a 5:1 uptake ratio, stable motion, a $5 \mathrm{~Hz}$ sampling rate, a $1000 \mu$ Gy fluoroscopic dose, and five bins. The studied reconstruction options have settings equal to those of this standard vector field compensation unless specified otherwise.

We investigated the cases of two, three, five, and 10 bins. Even for only two bins, the CNR was boosted significantly in comparison with no motion compensation. Using additional bins improved the CNR, but this increase was much smaller. This is because the magnitude of motion (average diaphragm amplitude of $10 \mathrm{~mm}$ for stable breathing) is in the same order of magnitude as the resolution of the gamma camera $(\sim 7.5 \mathrm{~mm}$ at $10 \mathrm{~cm})$ [30]. Accordingly, the mismatch in timeaveraged nuclear data with snap-shot fluoroscopic projections does not significantly influence the reconstruction quality.

The fluoroscopic dose levels were varied from 1-1000 $\mu \mathrm{Gy}$. A fluoroscopic dose of $10 \mu \mathrm{Gy}$ boosted the CNR considerably compared to a $1 \mu \mathrm{Gy}$ dose. However, an increase in the fluoroscopic dose did not further substantially improve the results.

The $\mathrm{x}$-ray sampling rate was varied between $1,2,3$, and $5 \mathrm{~Hz}$ at a total constant fluoroscopic dose level of $10 \mu \mathrm{Gy}$. This fluoroscopic dose level was chosen, as it was shown that this is the threshold for vector field motion compensation to function. The highest CNR was achieved at a fluoroscopic sampling rate of $2 \mathrm{~Hz}$. Fluoroscopic noise started to dominate with a further increase in the sampling rate, thus decreasing the $\mathrm{CNR}$. 
Table 1: Maximum CNR values for the several reconstruction options for the five-minute scan. No compensation resulted in a maximum CNR of $13.4 \pm 0.4$. No motion resulted in a maximum CNR of $26.5 \pm 0.9$.

\begin{tabular}{|c|c|c|c|c|c|}
\hline Number of bins & CNR & Fluoroscopic dose & CNR & Sampling rate & CNR \\
\hline 2 bins & $21.4 \pm 0.6$ & $1 \mu \mathrm{Gy}$ & $16.8 \pm 1.2$ & $1 \mathrm{~Hz}$ & $22.0 \pm 0.9$ \\
\hline 3 bins & $21.4 \pm 0.7$ & $10 \mu \mathrm{Gy}$ & $21.1 \pm 0.7$ & $2 \mathrm{~Hz}$ & $22.6 \pm 1.0$ \\
\hline 5 bins & $22.3 \pm 0.7$ & $100 \mu \mathrm{Gy}$ & $22.3 \pm 0.8$ & $3 \mathrm{~Hz}$ & $22.4 \pm 1.3$ \\
\hline 10 bins & $22.4 \pm 0.8$ & $1000 \mu \mathrm{Gy}$ & $22.3 \pm 0.7$ & $5 \mathrm{~Hz}$ & $21.1 \pm 0.7$ \\
\hline
\end{tabular}

The effect of using a motion vector field from a previously acquired 4D scan (with an inaccuracy of a few $\mathrm{mm}$ in the motion amplitudes), instead of using the vector field from the scan of interest, resulted in a CNR of $21.5 \pm 0.7$ instead of $22.3 \pm 0.7$.

The effects of irregular breathing motion were evaluated by performing reconstruction with several breathing patterns. These results are shown in Tab. 2. The reconstruction quality was largely unaffected by phase changes. The reconstruction quality increasingly deteriorated for amplitude changes, small variations, and baseline shifts. Vector field motion compensation led to minimal improvements in the reconstruction quality for large motion pattern variations, but yet it did not reduce performance more than when no motion compensation was applied. This suggests that the proposed motion compensation can always be performed since reconstruction quality did not decrease in the studied situations.

Finally, the difference in the detectability of regions with high activity using the two motion compensation methods was evaluated by reconstructing phantoms with different uptake ratios. These results are shown in Tab. 3. If the level of detectability is set at the Rose criterion of a CNR level of 4 [36], the spheres with a 2:1 uptake ratio would not be detected using gating. Conversely, this sphere could be observed using motion vector field compensation.

\section{Discussion}

In this study, we evaluated whether motion compensation using simultaneous fluoroscopic and nuclear imaging could enhance the SPECT quality in fast interventional liver scanning.

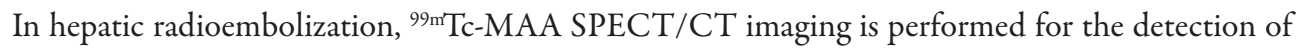
inadvertent regions of high activity and for the treatment planning of dosimetry. We have shown that regions with low uptake ratios can be better detected with our motion compensation method than with gating. This argument similarly holds for regions of increasingly smaller sizes. We acknowledge that high visual quality is currently not crucial for dosimetry, as activity recovery remains accurate for low count rates as with gating [37]. However, it could be argued that uniformity will become an increasingly important measure when voxel-based dosimetry [38] is further developed, as the activity will then also need to be correctly measured for small regions.

Two limitations of this study were that a phantom with rigid-like liver motion was evaluated and 
Table 2: Maximum CNR values for the irregular breathing patterns for the five-minute scan for no compensation, gating, and use of the motion vector field. No motion resulted in a maximum CNR of $26.5 \pm 0.9$.

\begin{tabular}{l|ccc} 
Breathing pattern & No compensation & Gating & Vector field \\
\hline Stable & $13.4 \pm 0.4$ & $15.1 \pm 0.8$ & $22.3 \pm 0.7$ \\
Phase change & $13.7 \pm 0.7$ & $15.4 \pm 1.0$ & $21.8 \pm 0.9$ \\
Amplitude change & $11.6 \pm 0.6$ & $15.5 \pm 0.7$ & $20.7 \pm 0.9$ \\
Baseline shift & $8.8 \pm 0.3$ & $12.7 \pm 0.7$ & $17.7 \pm 0.7$ \\
Small variations & $8.6 \pm 0.4$ & $14.0 \pm 1.0$ & $18.5 \pm 0.7$ \\
Large variations & $11.0 \pm 0.4$ & $10.4 \pm 0.7$ & $13.3 \pm 0.7$
\end{tabular}

that the proposed method assumed a non-deformable liver. The method discussed in this work is furthermore heavily dependent on the visibility of the lung/liver-barrier to extract the motion vector field. Assumptions have been made on the liver displacement in relation to the other organs, the validity of which will be studied in future work. For now, the effect of motion compensation should be carefully monitored in the case of regions of high activity further away from the liver. While the current implementation of the motion vector field requires a manual selection of regions to extrapolate the lung motion to the liver motion, this step should be automated once the system has passed the prototype phase. This is expected to be feasible because the boundaries between lung, air, and soft tissues are well discernible.

The background noise in gating could, to some extent, be compensated for in the reconstruction using regularizers, such as total variation minimization [39]. The smoothing of uniform regions, while preserving sharp edges, would improve the results on CNR similarly to the proposed method. However, since activity distributions will vary substantially between patients, the best setting for the required regularization parameter is often not evident. The use of optimal gating [40] could similarly provide smoother reconstructions but will come at the cost of a loss in resolution.

The prototype dual-layer detector system $[15,16]$ is currently being integrated into a custom C-arm. In the present simulation study, it was assumed that the flat panel could be decreased in thickness and attenuation by relocating the detector electronics. The camera size was additionally increased, and the detector orbit was reduced so that a closer orbit around the patient could be realized. $\mathrm{X}$-ray overflow on the gamma camera was not considered since this is not a limiting factor at low fluoroscopic doses $[41,42]$.

The simultaneous anatomic and nuclear information needed to acquire a motion vector field might also be successfully obtained using integrated SPECT/MRI [43], which does not have the downside of delivering radiation doses to patients and might better cope with irregular breathing. The main benefit of the proposed detector is that it is relatively small and mobile, and thus suited for interventional procedures. Additionally, by designing the gamma camera as an add-on to a regular c-arm, the manufacturing cost can be kept low and implementation in the clinic can be accelerated. 
Table 3: Maximum CNR values for the spheres with varied uptake ratios for the five-minute scan for no compensation, gating, and use of the motion vector field.

\begin{tabular}{c|ccc} 
Uptake ratio & No compensation & Gating & Vector field \\
\hline $2: 1$ & $2.7 \pm 0.3$ & $3.2 \pm 0.4$ & $4.4 \pm 0.5$ \\
$3: 1$ & $6.4 \pm 0.4$ & $7.1 \pm 0.6$ & $9.9 \pm 0.5$ \\
$4: 1$ & $10.0 \pm 0.5$ & $11.0 \pm 1.0$ & $15.7 \pm 1.2$ \\
$5: 1$ & $13.4 \pm 0.5$ & $15.1 \pm 0.8$ & $22.3 \pm 0.7$
\end{tabular}

A radiation dose is required for our approach to motion compensation. However, it is not expected that this will limit clinical use. First, a radiation dose will always be required for interventional scanning, inasmuch as the SPECT scan needs either a self-recorded attenuation map or a volume to perform registration on. The ability to perform motion compensation can thus be considered an added benefit. Second, it was shown that our approach required only $10 \mu \mathrm{Gy}$ for the described situation. This is very low in comparison with, e.g., the generation of an attenuation map in SPECT, which is usually in the order of several $\mathrm{mGy}$ [44]. And third, in the proposed application of hepatic radioembolization, patients locally receive several hundred Gy, many orders of magnitude more than in our approach.

Breathing motion patterns consist of a combination of phase changes (breathing faster or slower), amplitude changes (breathing more/less deeply), and baseline shifts (switching of breathing pattern) [45]. Algorithms such as the Amsterdam Shroud [46], Fourier-based approaches [47], and principle component analysis [48] cannot determine these amplitude and baseline changes. Furthermore, approaches using template matching $[49,50]$ require high-contrast images, which are not available if the administered fluoroscopic dose is kept low.

It was shown that changes in respiratory motion degrade the reconstruction results. However, the extent to which such motion variations will be present in an interventional (e.g., radioembolization) setting is unclear, as we unfortunately do not have access to patient respiratory signals. Patients are generally already on the operating table for some time before the scout scan is performed, which might reduce the likelihood of a change from an active to a resting state. Additionally, patients are often sedated to some degree, which will reduce stress-related breathing. For now, we assume that the motion vector field can be approximated as stable during a single scan. Future studies are needed to determine whether this assumption holds.

The prototype as described performs a single rotation only and is thus subject to the limited angle effect since projections are only available under certain angles [51]. One way to better cope with the influence of irregular breathing would be to perform multiple rotations during the scan. Faster rotation, however, requires better mechanical stability, which may not be achieved in the prototype under construction. This option has therefore not been further explored in this study but could be beneficial for future designs.

As discussed previously, an approximation of the motion vector field could be estimated with other modalities, such as CT or MRI. A major advantage of our motion compensation approach is that 
it is not sensitive to potential changes in breathing between scans, making it a reliable option for clinical practice. The above options would also require that the previously acquired $4 \mathrm{D}$ scan be matched to the SPECT scan, requiring a registration step. This registration could result in blurring, which is an additional disadvantage of using the vector field from a previously acquired 4D scan. The integrated SPECT/CBCT system captures the same field of view and hence does not have this problem.

By assigning nuclear counts to gates using the motion signal from an external gating device, it might be possible to estimate the motion vector field from the resulting reconstructed SPECT volumes. This approach has the benefit of not requiring any other modality for its motion compensation, and thus not requiring any hardware modifications. However, for fast scanning, we expect that the quality of the SPECT volumes will be severely limited by counting statistics, making it very challenging to estimate a correct vector field. While this approach might thus be beneficial for general motion compensation in SPECT, it is unlikely to succeed in a time-critical interventional setting.

It is not expected that the use of an anti-scatter grid will improve the detection of the lung-liver barrier since the difference in density between these tissues is relatively large. However, when simultaneously applying a higher fluoroscopic dose, the soft-tissue contrasts in the reconstructions may become more apparent. Tracking all organs individually may then still be challenging but should be feasible with the use of dedicated registration software. Such an approach would additionally allow deformable registration, in contrast to the proposed method, which assumes a rigid liver. Our aim is to limit the fluoroscopic dose rate initially and evaluate its performance. If it proves that the used extrapolation method does not accurately describe the respiratory motion, the option above could be further explored.

Three steps in the total reconstruction process take up most of the reconstruction time. For a single bin, and with a single thread on a regular desktop computer, the required times are approximately 10 seconds for $\mathrm{CBCT}$ reconstruction, two minutes for registration, and 30 minutes for SPECT reconstruction (for 25 iterations). All bins can be processed in parallel, which means that the total number of bins will generally not be limiting. With code optimization and the use of dedicated hardware, we expect that a total reconstruction time of five minutes, fast enough for use in an interventional setting, can be achieved.

\section{Conclusion}

We have developed and evaluated a motion compensation approach using simultaneous fluoroscopic and nuclear imaging with a dual-layer detector. Such a configuration can intrinsically measure the respiratory motion signal and the associated motion vector field during a SPECT scan, which eliminates the need for external devices and provides an optimal motion vector field. The SPECT reconstruction quantitative accuracy is boosted substantially compared with no motion correction; the visual quality is also improved compared to gating. Combined with the fast reconstruction implementation, this will allow for motion-corrected scans in the intervention room. 


\section{References}

1. Bastiaannet R, Viergever MA, De Jong HWAM. Impact of respiratory motion and acquisition settings on SPECT liver dosimetry for radioembolization. Med. Phys. 44(10): 5270-5279 (2017)

2. Pretorius PH, Johnson KL, Dahlberg ST, King MA. Investigation of the physical effects of respiratory motion compensation in a large population of patients undergoing Tc- $99 \mathrm{~m}$ cardiac perfusion SPECT/ CT stress imaging, J Nucl Cardiol: 1-16 (2017)

3. Turner DA, Fordham EW, Ali A, et al. Motion corrected hepatic scintigraphy: an objective clinical evaluation. J. Nucl. Med. 19(2):142-148 (1978)

4. Cho K, Kumiata SI, Okada S, Kumazaki T. Development of respiratory gated myocardial SPECT system. J. Nucl. Cardiol. 6(1):20-28 (1999)

5. McClelland JR, Hawkes DJ, Schaeffter T, King AP. Respiratory motion models: A review. Med. Image Anal. 17(1):19-42 (2013)

6. Li T, Thorndyke B, Schreibmann E, Yang Y, Xing L. Model-based image reconstruction for fourdimensional PET. Med. Phys. 33(5):1288-1298 (2006)

7. Pan T, Lee TY, Rietzel E, Chen GTY. 4D-CT imaging of a volume influenced by respiratory motion on multi-slice CT. Med. Phys. 31(2):333-340 (2004)

8. Schleyer PJ, O'Doherty MJ, Barrington SF, Marsden PK. Retrospective data-driven respiratory gating for PET/CT. Phys. Med. Biol. 54(7):1935-1950 (2009)

9. Sanders JC, Ritt P, Kuwert T, Vija AH, Maier AK. Fully automated data-driven respiratory signal extraction from SPECT images using Laplacian eigenmaps. IEEE Trans. Med. Im. 35(11):2425-2435 (2015)

10. Nehmeh SA, Erdi YE. Respiratory Motion in Positron Emission Tomography/Computed Tomography: A Review. Semin. Nucl. Med. 38(3):167-176 (2008)

11. Rohlfing T, Maurer CR, O’Dell WG, Zhong J. Modeling liver motion and deformation during the respiratory cycle using intensity-based nonrigid registration of gated MR images. Med. Phys. 31(3):427$432(2004)$

12. Yang D, Lu W, Low DA, Deasy JO, Hope AJ, El Naqa I. 4D-CT motion estimation using deformable image registration and 5D respiratory motion modeling. Med. Phys. 35(10):4577-4590 (2008)

13. King AP, Buerger C, Tsoumpas C, Marsden PK, Schaeffter T. Thoracic respiratory motion estimation from MRI using a statistical model and a 2-D image navigator. Med. Image Anal. 16(1):252-264 (2012)

14. Redmond KJ, Song DY, Fox JL, Zhou J, Rosenzweig CN, Ford E. Respiratory Motion Changes of Lung Tumors Over the Course of Radiation Therapy Based on Respiration-Correlated Four-Dimensional Computed Tomography Scans. Int. J. Radiat. Oncol. Biol. Phys. 75(5):1605-1612 (2009)

15. Van der Velden S, Kunnen B, Koppert WJC, et al. A Dual-layer Detector for Simultaneous Fluoroscopic and Nuclear Imaging. Radiology 290(3):833-838 (2019)

16. Dietze MMA, Kunnen B, Van der Velden D, Steenbergen JHL, Koppert WJC, Viergever MA, De Jong HWAM. Performance of a dual-layer scanner for hybrid SPECT/CBCT. Phys. Med. Biol. 64(10):105020 (2019)

17. Vedam SS, Keall PJ, Kini VR, Mostafavi H, Shukla HP, Mohan R. Acquiring a four-dimensional computed tomography dataset using an external respiratory signal. Phys. Med. Biol. 48(1):45-62 (2003)

18. Braat ATAJ, Smits MLJ, Braat MNGJA, et al. $Y^{90}$ Hepatic Radioembolization: An Update on Current Practice and Recent Developments. J. Nucl. Med. 56(2), 1079-1087 (2015)

19. Gabr A, Kallini JR, Gates VL, et al. Same-day ${ }^{90} \mathrm{Y}$ radioembolization: implementing a new treatment 
paradigm. Eur. J. Nucl. Med. Mol. Imaging 43(13):2353-2359 (2016)

20. Segars WP, Sturgeon G, Mendonca S, Grimes J, Tsui BMW. 4D XCAT phantom for multimodality imaging research. Med. Phys. 37(9):4902 (2010)

21. Vedam SS, Kini VR, Keall PJ, Ramakrishnan V, Mostafavi H, Mohan R. Quantifying the predictability of diaphragm motion during respiration with a noninvasive external marker. Med. Phys. 30(4):505-513 (2003)

22. Jan S, Santin G, Strul D, et al. GATE: A simulation toolkit for PET and SPECT. Phys. Med. Biol. 49(19):4543-4561 (2004)

23. Poludniowski G, Evans PM, Hansen VN, Webb S. An efficient Monte Carlo-based algorithm for scatter correction in keV cone-beam CT, Phys. Med. Biol. 54(12):3847-3864 (2009)

24. Siemens. Online tool for the simulation of X-ray Spectra. https://www.oem-xray-components.siemens. com/x-ray-spectra-simulation

25. De Jong HWAM, Slijpen ETP, Beekman FJ. Acceleration of Monte Carlo SPECT simulation using convolution-based forced detection. IEEE Trans. Nucl. Sci. 48(1):58-64 (2001)

26. Xiao J, De Wit TC, Staelens SG, Beekman FJ. Evaluation of 3D Monte Carlo-based scatter correction for ${ }^{99 m}$ Tc cardiac perfusion SPECT. J. Nucl. Med. 47(10):1662-9 (2006)

27. Elschot M, Lam MGEH, Van den Bosch MAAJ, Viergever MA, De Jong HWAM. Quantitative Monte Carlo-based ${ }^{90}$ Y SPECT reconstruction. J. Nucl. Med. 54(9):1557-63 (2013)

28. Elschot M, Smits MLJ, Nijsen JFW, et al. Quantitative Monte Carlo-based holmium-166 SPECT reconstruction. Med. Phys. 40(11):112502 (2013)

29. Dietze MMA, Van der Velden S, Lam MGEH, Viergever MA, De Jong HWAM. Fast quantitative reconstruction with focusing collimators for liver SPECT. EJNMMI Phys 5(1):28 (2018)

30. Siemens. Symbia $S$ and T System Specifications. (2010)

31. Zhang H, Ouyang L, Ma J, Huang J, Chen W, Wang J. Noise correlation in CBCT projection data and its application for noise reduction in low-dose CBCT. Med. Phys. 41(3):031906 (2014)

32. Biguri A, Dosanjh M, Hancock S, Soleimani M. TIGRE: a MATLAB-GPU toolbox for CBCT image reconstruction. Biomed. Phys. Eng. Express 2(5):055010 (2016)

33. Feldkamp LA, Davis LC, Kress JW. Practical cone-beam algorithm. J. Opt. Soc. Am. A 1(6):612 (1984)

34. Klein S, Staring M, Murphy K, Viergever MA, Pluim JPW. Elastix: A toolbox for intensity-based medical image registration. IEEE Trans. Med. Imaging 29(1):196-205 (2010)

35. Sonke JJ, Lebesque J, Van Herk M. Variability of Four-Dimensional Computed Tomography Patient Models. Int. J. Radiat. Oncol. Biol. Phys. 70(2):590-598 (2008)

36. Rose A. The sensitivity performance of the human eye on an absolute scale. J. Opt. Soc. Am. 38(2):196208 (1948)

37. Van der Velden S, Dietze MMA, Viergever MA, De Jong HWAM. Fast technetium-99m liver SPECT for evaluation of the pre-treatment procedure for radioembolisation dosimetry. Med Phys. 46(1):345$355(2019)$

38. Mikell JK, Mahvash A, Siman W, Mourtada F, Kappadath SC. Comparing voxel-based absorbed dosimetry methods in tumors, liver, lung, and at the liver-lung interface for ${ }^{90} \mathrm{Y}$ microsphere selective internal radiation therapy. EJNMMI Phys. 2(1):16 (2015)

39. Panin VY, Zeng GL, Gullberg GT. Total variation regulated EM algorithm. IEEE Trans. Nucl. Sci. 46(6):2202-2210 (1999)

40. Van Elmpt W, Hamill J, Jones J, De Ruysscher D, Lambin P, Öllers M. Optimal gating compared to 
3D and 4D PET reconstruction for characterization of lung tumours. Eur. J. Nucl. Med. Mol. Imaging 38(5):843-855 (2011)

41. Koppert WJC, Van der Velden S, Steenbergen JHL, De Jong HWAM. Impact of intense x-ray pulses on a NaI(Tl)-based gamma camera. Phys. Med. Biol. 63(6):065006 (2018)

42. Koppert WJC, Dietze MMA, Van der Velden S, Steenbergen JHL, De Jong HWAM. A Comparative Study of $\mathrm{NaI}(\mathrm{Tl}), \mathrm{CeBr}_{3}$, and CZT for use in a Real-Time Simultaneous Nuclear and Fluoroscopic DualLayer Detector. Phys. Med. Biol. 64(13):135012 (2019)

43. Hutton BF, Occhipinti M, Kuehne A, et al. Development of clinical simultaneous SPECT/MRI. Br. J. Radiol. 91(1081):20160690 (2018)

44. Rausch I, Füchsel FG, Kuderer C, Hentschel M, Beyer T. Radiation exposure levels of routine SPECT/ CT imaging protocols. Eur. J. Radiol. 85(9):1627-1636 (2016)

45. Fassi A, Schaerer J, Fernandes M, Riboldi M, Sarrut D, Baroni G. Tumor tracking method based on a deformable 4D CT breathing motion model driven by an external surface surrogate. Int. J. Radiat. Oncol. Biol. Phys. 88(1):182-188 (2014)

46. Sonke JJ, Zijp L, Remeijer P, Van Herk M. Respiratory correlated cone beam CT. Med. Phys. 32(4):1176$1186(2005)$

47. Vergalasova I, Cai J, Yin FF. A novel technique for markerless, self-sorted 4D-CBCT: Feasibility study. Med. Phys. 39(3):1442-1451 (2012)

48. Hao Y, Xiaoyu W, Wotao Y, et al. Extracting respiratory signals from thoracic cone beam CT projections. Phys. Med. Biol. 58(5):1447 (2013)

49. Rit S, Sarrut D, Ginestet C. Respiratory Signal Extraction for 4D CT Imaging of the Thorax from ConeBeam CT Projections. MICCAI:556-563 (2015)

50. Park JC, Park SH, Kim JH, et al. Four-dimensional cone-beam computed tomography and digital tomosynthesis reconstructions using respiratory signals extracted from transcutaneously inserted metal markers for liver SBRT. Med. Phys. 38(2):1028-1036 (2011)

51. Dey J, Segars WP, Pretorius PH, et al. Estimation and correction of cardiac respiratory motion in SPECT in the presence of limited-angle effects due to irregular respiration. Med. Phys. 37(12):6453-6465 (2010) 



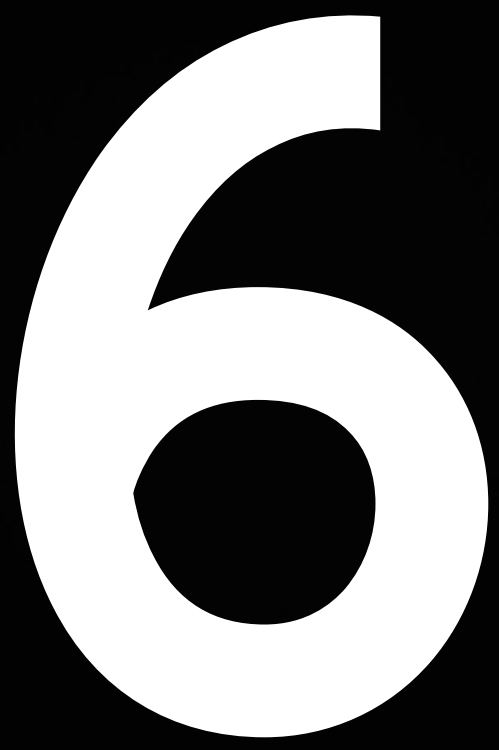




\section{INTERVENTIONAL RESPIRATORY MOTION COMPENSATION VALIDATION}

BASED ON:

MMA Dietze, B Kunnen, MGEH Lam, HWAM de Jong Interventional Respiratory Motion Compensation by Simultaneous Fluoroscopic and Nuclear Imaging 


\section{Abstract}

\section{Purpose}

A compact and mobile hybrid c-arm scanner, capable of simultaneously acquiring nuclear and fluoroscopic projections and SPECT/CBCT, was developed to aid fluoroscopyguided interventional procedures involving the administration of radionuclides (e.g. hepatic radioembolization). However, as in conventional SPECT/CT, the acquired nuclear images may be deteriorated by patient respiratory motion. We propose to perform compensation for respiratory motion by extracting the motion signal from low-dose fluoroscopic projections that are simultaneously acquired with the nuclear data so that the nuclear counts can be gated into motion bins. The purpose of this study is to evaluate this motion compensation technique with phantom experiments.

\section{Methods}

Anthropomorphic phantom configurations that are representative of distributions obtained during the pre-treatment procedure of hepatic radioembolization were placed on a translating stage that mimicked respiratory motion. Low-dose fluoroscopic projections and nuclear counts were simultaneously acquired under planar and SPECT/CBCT imaging while the phantom translated and while it was stationary. The planar projections were visually assessed. The SPECT reconstructions were visually assessed and quantitatively assessed by calculating the activity recovery of the spherical inserts in the phantom.

\section{Results}

The planar nuclear projections of the translating anthropomorphic phantom were blurry when no motion compensation was applied. With motion compensation, the nuclear projections became sharper and representative of the stationary phantom nuclear projection. The same behavior was observed for the SPECT reconstructions. The mean activity recovery of one of the spherical inserts in the SPECT reconstructions was $53.5 \pm 0.4 \%$ when no motion compensation was applied. With motion compensation, the activity recovery improved to $64.7 \pm 0.8 \%$. This activity recovery is similar to the recovery that was obtained for the stationary anthropomorphic phantom $(65.2 \pm$ $0.4 \%)$.

\section{Conclusion}

A compact and mobile hybrid c-arm scanner, capable of simultaneously acquiring nuclear and fluoroscopic projections, can perform compensation for respiratory motion. Such motion compensation improves the interpretation of planar nuclear projections and increases the quantitative accuracy of the SPECT reconstructions. 


\section{Introduction}

Fluoroscopy-guided interventions involving the administration of radionuclides (e.g. hepatic radioembolization [1] or sentinel node procedures [2]) would benefit from acquiring nuclear images while in the intervention room since this would allow for the evaluation of the activity distribution during the procedure. Recently, a compact and mobile hybrid c-arm scanner was developed that enables: i) procedure guidance by simultaneous acquisition of planar nuclear and fluoroscopic projections with the same field of view, and ii) procedural dosimetry by the acquisition of hybrid SPECT/CBCT reconstructions [3,4]. We refer to this novel scanner as IXSI (Interventional X-ray and Scintigraphy Imaging).

The quality and the quantitative accuracy of the nuclear images that will be obtained during interventional procedures may be deteriorated due to patient respiratory motion. For planar imaging, a heavy respiratory motion will result in blurred nuclear projections, since the nuclear counts are smeared out over several pixels. For SPECT/CBCT imaging, a heavy respiratory motion will cause deteriorated dosimetric quality [5]. The procedure guidance capability of IXSI would hence improve when accurate compensation for respiratory motion is made.

The most common approach to compensate for respiratory motion is to gate the nuclear counts (obtained in list-mode) in motion bins by referencing a measure of the motion signal over time. This motion signal can be retrieved from external devices (e.g. a respiratory belt) or from data-driven approaches (e.g. tracking the center-of-mass of the nuclear counts) [6]. However, external devices are undesired in an interventional setting since they are difficult to work with and do not provide sufficient flexibility. Data-driven approaches are often unstable when imaging small activity doses since these methods rely heavily on the counting statistics.

These conventional methods for obtaining the motion signal are hence not best suited to be employed in interventional procedures. We propose a methodology that does meet the requirements for interventional imaging: by tracking the motion in the fluoroscopic projections, a continuously stable estimate of the motion signal is obtained that does not require the setup of external devices. A downside of the proposed approach is that extra dose is administered to the patient but it will be demonstrated that this dose can be kept very low.

By employing the motion compensation approach with IXSI, the projections obtained with planar imaging are expected to become sharper and the quantitative accuracy of the SPECT reconstruction should improve. Before IXSI will be employed in patients, this study aimed to quantify the performance of the proposed motion compensation approach with phantom experiments.

\section{Methods}

\section{IXSI}

IXSI consists of a detector stack that is positioned on a mobile custom-made c-arm (Indes, Enschede, The Netherlands) with an x-ray tube (Veradius; Philips Healthcare, Best, The Netherlands) 

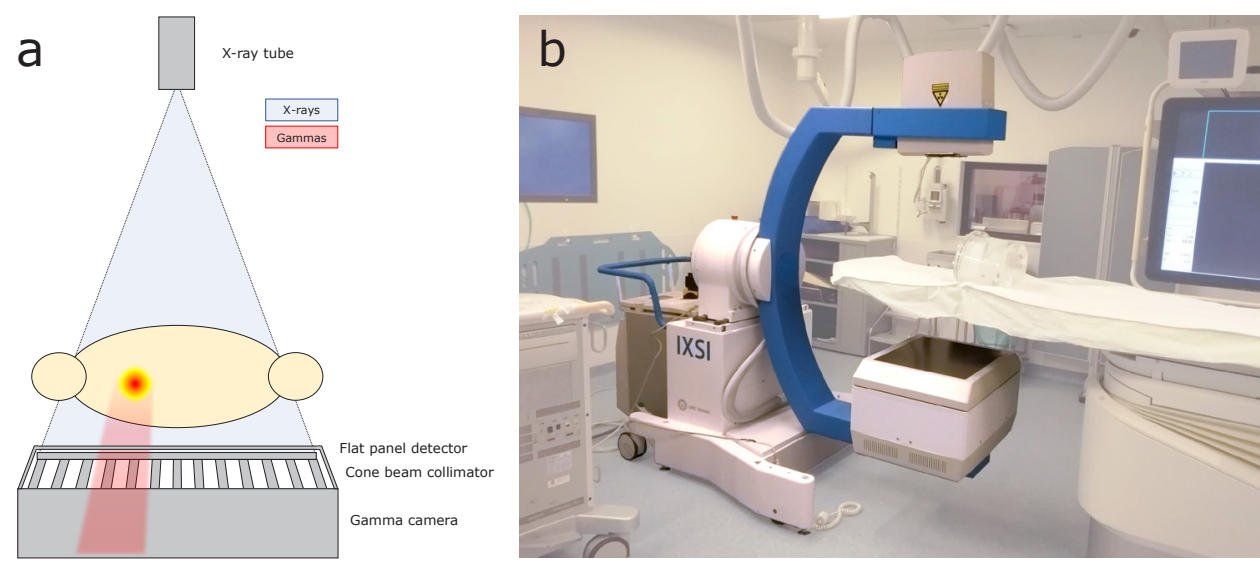

Figure 1: (a) A schematic illustration of the scanner components and (b) the developed scanner. IXSI consists of a detector stack (consisting of a collision detector, a flat panel detector, a cone beam collimator, and a gamma camera) that is mounted together with the $\mathrm{x}$-ray tube on a mobile custom c-arm.

positioned on the opposite side (see Fig. 1). The detector stack consists of a CBCT flat panel detector (Pixium 3040; Trixell, Moirans, France) that is positioned in front of a gamma camera (P3000; Inter Medical, Lübbecke, Germany) that is mounted with a cone beam collimator (Nuclear Fields, Vortum-Mullem, The Netherlands). The x-ray tube is positioned at the same distance as the focal distance of the cone beam collimator $(105 \mathrm{~cm})$ so that intrinsically overlapping nuclear and fluoroscopic projections are acquired.

The c-arm can translate in two directions while rotating and is able to perform parameterized (noncircular) orbits. A collision detector was present in front of the flat panel detector so that the c-arm rotation terminated when contact with an object was registered. The settings of the $\mathrm{x}$-ray tube were set on the console that is integrated into the scanner.

The flat panel detector was adjusted from the commercially available product by reduction of the back-shielding thickness and by removal of several internal highly-attenuating components (e.g. support screws) so that the transmission of gamma photons was increased. The thickness of the flat panel detector was $6.8 \mathrm{~cm}$ and the average transmission of gamma photons at $140 \mathrm{keV}$ was $52 \%$. The field of view (FOV) of the flat panel detector was $39.9 \times 29.5 \mathrm{~cm}^{2}$.

The gamma camera was adjusted from the commercially available product by having alterations in the detector housing to fit on the custom c-arm. The effective FOV of the gamma camera was 51.0 $\mathrm{x} 38.1 \mathrm{~cm}^{2}$. The cone beam collimator had holes with $40.0 \mathrm{~mm}$ length, $1.90 \mathrm{~mm}$ inner diameter, and $0.25 \mathrm{~mm}$ septal thickness. The gamma camera had an intrinsic spatial resolution of $3.9 \mathrm{~mm}$ full width at half maximum (FWHM) and an energy resolution of $9.4 \%$. 

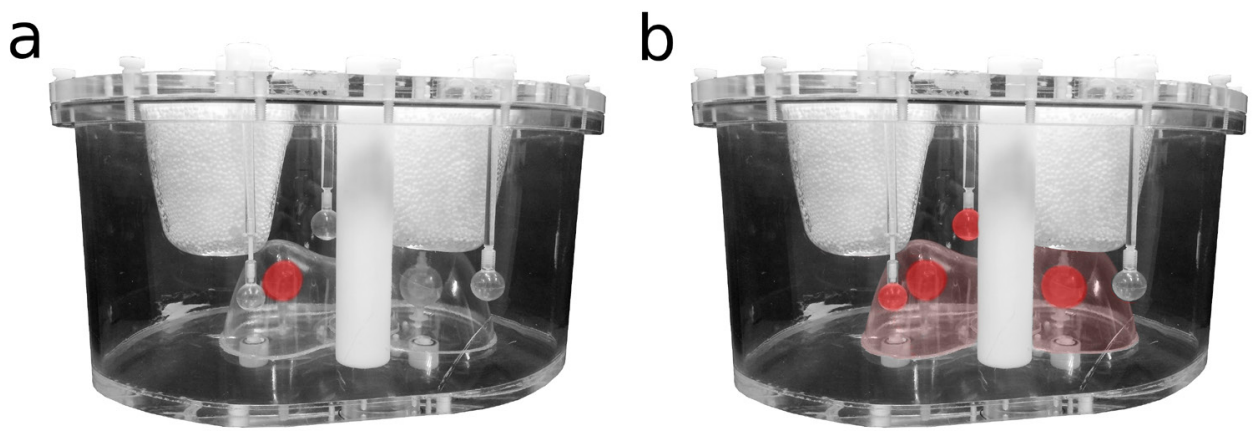

Figure 2: A representation of the two activity distributions that were evaluated: (a) a superselective and (b) a whole liver treatment. The red overlay represents the volumes that had activity present.

\section{Phantoms}

One of the proposed uses of IXSI is the guidance of the pre-treatment procedure of hepatic radioembolization, in which ${ }^{99} \mathrm{~m} T \mathrm{~T}$-labeled macro aggregated albumin $\left({ }^{99} \mathrm{~m} T \mathrm{~T}-\mathrm{MAA}\right)$ is injected into the hepatic artery. Two activity distributions (see Fig. 2) that are expected to arise during the procedure guidance of radioembolization were evaluated:

\section{Superselective treatment}

The first activity distribution represents an interventional procedure in which only one tumor is targeted (i.e. a superselective treatment) and all activity accumulates in this tumor. This setting was recreated by inserting a sphere $(16 \mathrm{ml})$ of $38 \mathrm{MBq}$ in the liver compartment of an otherwise nonactive anthropomorphic thorax phantom (model ECT/TOR/P).

\section{Whole liver treatment}

The second activity distribution represents a whole liver treatment in which the activity has spread out. This setting was recreated by filling the liver compartment $(1172 \mathrm{ml})$ with $229.7 \mathrm{MBq}$, inserting a sphere $(16 \mathrm{ml})$ of $24.9 \mathrm{MBq}$ and a sphere with a cold core $(19 \mathrm{ml}$ active volume $)$ of $30.2 \mathrm{MBq}$ in the liver compartment (tumor to non-tumor ratio of 8:1), and inserting two spheres (8.1 and $4.1 \mathrm{ml}$ ) of respectively 4.8 and $2.5 \mathrm{MBq}$ in the background compartment (simulating extrahepatic accumulations).

\section{Acquisition}

For all experiments, fluoroscopic projections were sampled at $3.75 \mathrm{~Hz}$ and nuclear counts were simultaneously acquired in list-mode. The gamma camera counts that were obtained while the $\mathrm{x}$-ray tube was active were removed from the nuclear data since these counts could have originated from $\mathrm{x}$-ray photons instead of gamma photons $[7,8]$. The fluoroscopic projections were acquired at a 1008 x 1008 grid with a $0.399 \mathrm{~mm}$ pixel size. The nuclear projections were acquired at a $128 \times 128$ grid with a $4.79 \mathrm{~mm}$ pixel size. 

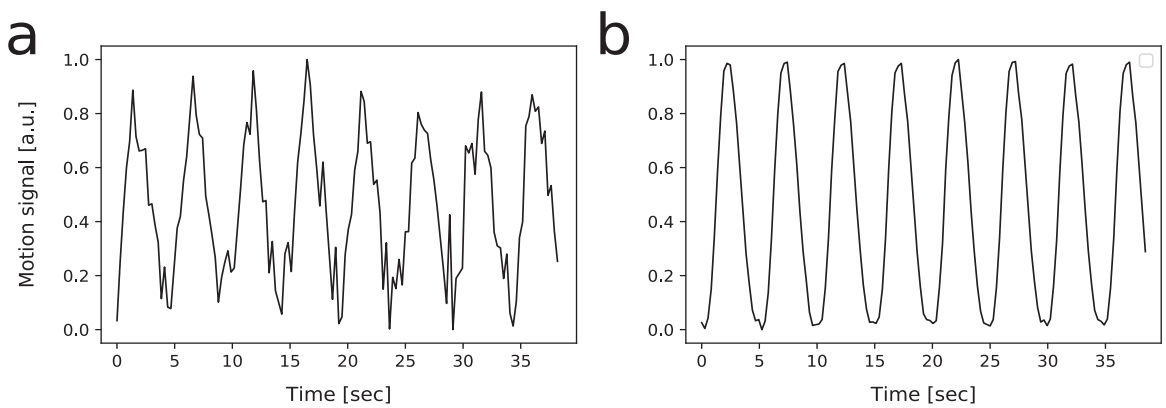

Figure 3: Examples of the extracted motion signals in arbitrary units (a.u.) for (a) the data-driven approach (with $35 \mathrm{MBq}$ ) and (b) the fluoroscopy-guided approach (with $60 \mathrm{kVp}$ ).

Both anthropomorphic phantom configurations were placed on a motor-driven stage that translated in one direction with a sinusoidal movement with maximum motion amplitude of $2 \mathrm{~cm}$ and a phase of 5 seconds. The motion compensation performance for planar imaging was studied with the superselective treatment phantom since this phantom configuration provides a sharp contrast in the planar images. The motion compensation performance for SPECT/CBCT imaging was studied with the whole liver treatment phantom configuration this phantom configuration has several features that are interesting to study quantitively.

\section{Motion signal extraction}

First, two methods were compared on their motion signal extraction: i) a data-driven approach, in which the center of mass of the nuclear counts was determined, and ii) the proposed fluoroscopyguided approach, in which the position of the diaphragm was tracked over the fluoroscopic projections. The motion signal extraction performance was studied for planar imaging with the superselective treatment phantom configuration. Pearson's correlation coefficient between the extracted motion signal and the true phantom position was calculated for both motion signal extraction methods. Every motion-signal extraction measurement was repeated five times to study the stability of the results.

To get insight into the influence of dose and activity on the signal extraction performance, the $\mathrm{x}$-ray tube strength was varied from 45 to $80 \mathrm{kVp}$ and the activity level in the phantom was varied from 5 to $35 \mathrm{MBq}$ (by sampling counts from the list-mode data). The dose corresponding to the above $\mathrm{X}$-ray tube settings was determined with the dose area product (DAP) meter that is integrated into IXSI. It will prove that the fluoroscopy-guided approach provides a more stable signal over the respiratory cycle than the data-driven approach. The remainder of the manuscript hence employed the fluoroscopy-guided approach for motion compensation.

\section{Planar imaging}

To assess the planar imaging performance, nuclear and fluoroscopic projections were simultaneously acquired for the anthropomorphic phantom with the superselective treatment configuration while it was translating and while it was stationary. The acquisition time was one minute for all scans. 

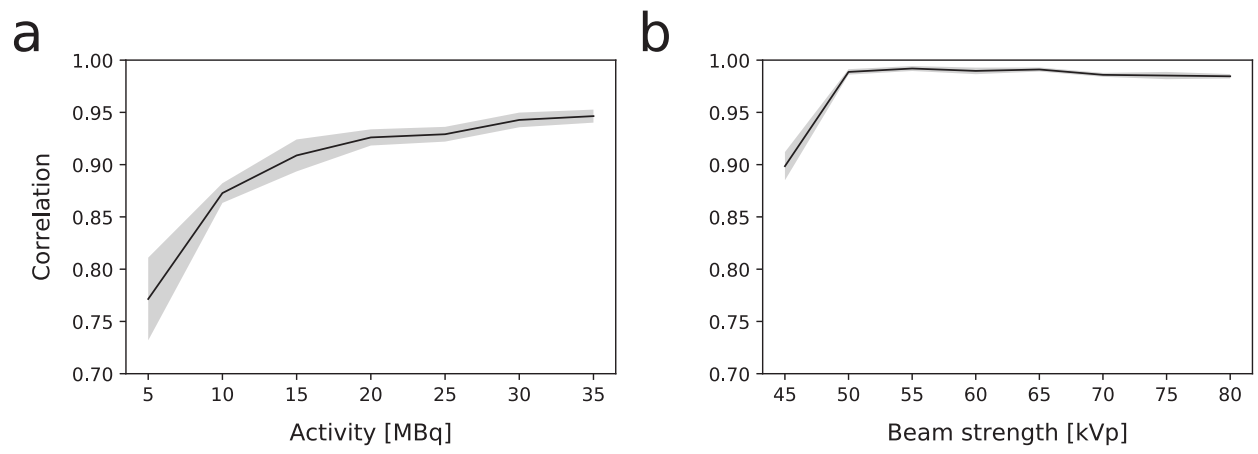

Figure 4: The correlation between the extracted motion signal and the true phantom position for (a) the datadriven approach as a function of the phantom activity and (b) the fluoroscopic projection approach as a function of the $\mathrm{x}$-ray tube strength.

For the translating phantom without motion compensation and for the stationary phantom, the nuclear projections were created by summing all nuclear counts together. For the translating anthropomorphic phantom with motion compensation, the following steps were taken: i) the motion signal was determined from the fluoroscopic projections, ii) the nuclear counts were gated into five motion bins based on the motion signal amplitude, and iii) the nuclear projection was created by including only the nuclear counts from one of the five motion bins (the bin that had the most nuclear counts present was selected). A $5 \mathrm{~mm}$ FWHM Gaussian filter was applied to the nuclear projections and the visual quality of the projections was compared.

\section{SPECT/CBCT imaging}

To assess the SPECT/CBCT imaging performance, nuclear and fluoroscopic projections were simultaneously acquired for the anthropomorphic phantom with the whole liver treatment configuration while it was translating and while it was stationary. The c-arm rotated over 360 degrees (with 120 angles) during an acquisition of 10 minutes, for all scans.

For the translating phantom without motion compensation and for the stationary phantom, all nuclear counts and fluoroscopic projections were processed together. For the translating phantom with motion compensation, the following steps were taken: i) the motion signal was extracted from the fluoroscopic projections, ii) the motion signal was normalized to the peaks (because the changing geometry and attenuation over the rotation introduced a position-dependent signal shift), iii) the nuclear counts and fluoroscopic projections were gated into five motion bins based on the normalized motion signal amplitude, and iv) one of the five motion bins (the one that had the most nuclear counts present) was selected for further processing.

The fluoroscopic projections were reconstructed using reconstruction software from Philips Healthcare. Since the CBCT reconstruction had a limited field of view and because the quality of the $\mathrm{CBCT}$ reconstruction was deteriorated by scattered photons, the $\mathrm{CBCT}$ reconstruction was registered to a previously acquired attenuation map using the elastix software package [9]. 
Table 1: The dose area product for the evaluated $x$-ray tube settings in DAP per minute and total DAP for 15 min of scanning.

\begin{tabular}{c|cc} 
Beam strength $[\mathrm{kVp}]$ & DAP $\left[\mu \mathrm{Gy} \mathrm{m}{ }^{2} / \mathrm{min}\right]$ & $15 \mathrm{~min} \mathrm{DAP}\left[\mathrm{mGy} \mathrm{m}^{2}\right]$ \\
\hline 60 & 51.9 & 0.8 \\
65 & 98.3 & 1.5 \\
70 & 188.3 & 2.8 \\
75 & 236.5 & 3.5 \\
80 & 284.6 & 4.3
\end{tabular}

The nuclear projections were reconstructed with the Utrecht Monte Carlo System (UMCS) software package, which included Monte Carlo-based scatter correction, point spread function (PSF) modeling, and attenuation correction $[10,11]$. The reconstructor used the OSEM reconstruction algorithm with 10 iterations and 8 subsets and reconstructed to a $128 \times 128 \times 128$ grid with a 4.79 $\mathrm{mm}$ isotropic voxel size. A $5 \mathrm{~mm}$ FWHM Gaussian post-reconstruction filter was applied to the reconstructions.

The total activity in the anthropomorphic phantom with the whole liver treatment configuration was $292 \mathrm{MBq}$. By sampling from the list-mode nuclear counts, nuclear projections corresponding to $150 \mathrm{MBq}$ total phantom activity were created (so that the same activity level as injected during a clinical pre-treatment procedure of radioembolization was achieved). The sampling process was repeated 10 times to study the influence of different noise patterns on the reconstruction stability.

The visual quality and the quantitative accuracy of the SPECT reconstructions were assessed. For the quantitative analysis, the activity recoveries of the four spherical inserts were calculated. This metric is important in e.g. dosimetry as required for radioembolization planning. The masks of the spherical inserts were manually delineated on the registered attenuation map.

\section{Results}

\section{Motion signal extraction}

Examples of the extracted motion signals for the data-driven (using $35 \mathrm{MBq}$ ) and fluoroscopyguided approach (using $60 \mathrm{kVp}$ ) are given in Fig. 3. The data-driven method had substantial variations in the motion signal amplitude over time. In contrast, the fluoroscopy-guided motion signal provided a very stable motion signal.

The correlation between the extracted motion signal and the true phantom position is shown in Fig. 4 for the data-driven approach as a function of the phantom activity and the fluoroscopic projection approach as a function of the $\mathrm{x}$-ray tube strength. The correlation from the fluoroscopic motion signal extraction was consistently higher than that of the data-driven approach. The correlation of the fluoroscopic projection approach reached a plateau after an $\mathrm{x}$-ray tube strength of $50 \mathrm{kVp}$. Because the fluoroscopic projection approach provided the most stable results, this method was used in the remainder of the manuscript. 

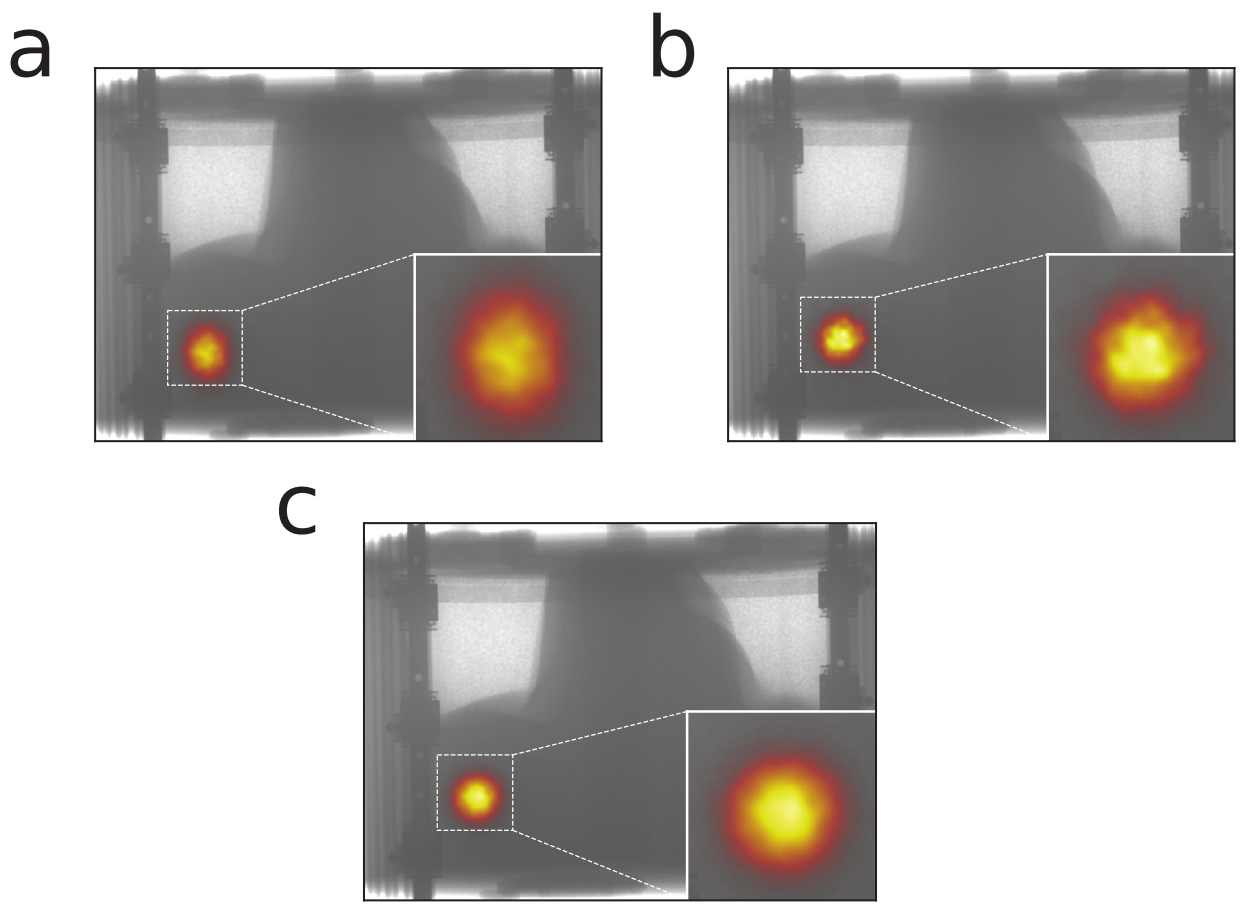

Figure 5: The nuclear projections obtained with planar imaging of the translating anthropomorphic phantom with the superselective treatment configuration when made (a) without and (b) with motion compensation. The projection obtained from the stationary phantom is shown in (c) as a reference.

The dose levels corresponding to the above-evaluated $\mathrm{x}$-ray tube settings are collected in Tab. 1 . The dose is provided in DAP per minute and DAP for a total of 15 minutes of scanning (e.g. 5 min of planar imaging and $10 \mathrm{~min}$ of SPECT/CBCT). The DAP-meter that is integrated into IXSI was not able to measure the dose below $60 \mathrm{kVp}$. Hence, although $50 \mathrm{kVp}$ was previously found to be sufficient for the accurate extraction of the motion signal, $60 \mathrm{kVp}$ was used for the fluoroscopic projections in the remainder of this work.

\section{Planar imaging}

The nuclear projections obtained through planar imaging of the translating anthropomorphic phantom with the superselective treatment configuration when made with and without motion compensation are shown together with the projection from the stationary anthropomorphic phantom in Fig. 5. The sphere was smeared out in case of the moving phantom with no motion compensation. When applying motion compensation, the sphere retrieved a similar shape as the sphere in the stationary anthropomorphic phantom. The motion-compensated moving phantom had more noise in its projection than the stationary phantom projection because of the counts that are disregarded when applying motion compensation. 


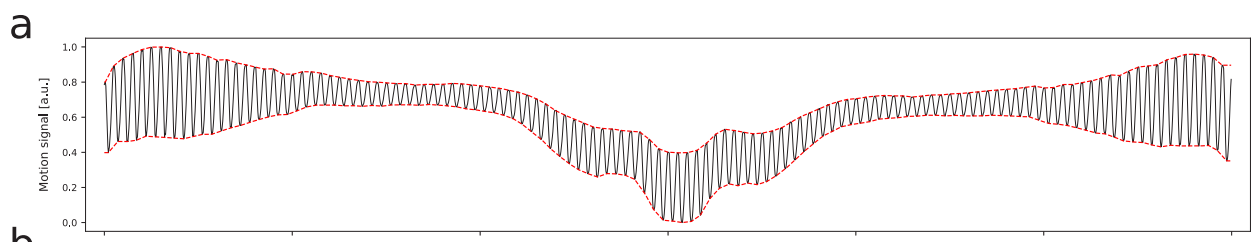

b

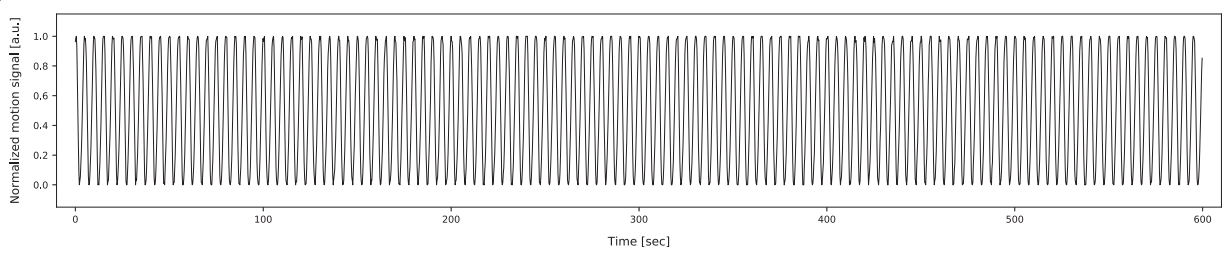

Figure 6: (a) The motion signal that was extracted while that scanner was rotating around the translating anthropomorphic phantom. The red dashed lines denote the lower and upper bounds of the signal. (b) The motion signal normalized to the upper and lower boundaries.

\section{SPECT/CBCT imaging}

The motion signal that is obtained during the rotation of the scanner around the translating anthropomorphic phantom is shown in Fig. 6a. This figure illustrates the dependence of the motion signal to the relative position of the scanner to the phantom. The upper and lower bounds of the motion signal were determined and the signal was normalized in Fig. 6b. This normalized signal was used to gate the nuclear counts into the five motion bins.

The SPECT reconstructions of the translating anthropomorphic phantom (whole liver treatment configuration) when made with and without motion compensation are shown together with the reconstruction of the stationary anthropomorphic phantom in Fig. 7. The reconstruction obtained without motion compensation $\left(1.26 \times 10^{6}\right.$ total counts in the projections) resulted in a blurry image. The reconstruction obtained with motion compensation $\left(0.42 \times 10^{6}\right.$ total counts $)$ was substantially sharper than the reconstruction obtained without motion compensation.

The quantitative evaluation of the SPECT reconstructions is collected in Tab. 2. No motion compensation resulted in relatively low activity recoveries for all spherical inserts (for the solid tumor: $53.5 \pm 0.4 \%$ ). Motion compensation substantially improved the activity recoveries (solid tumor: $64.7 \pm 0.8 \%)$ and resulted in similar activity recovery levels as in the stationary anthropomorphic phantom (solid tumor: $65.2 \pm 0.4 \%$ ). Hence, motion compensation also quantitatively substantially improved the SPECT reconstruction.

\section{Discussion}

This study demonstrated the potential of performing motion compensation using a hybrid c-arm scanner that is capable of simultaneous fluoroscopic and nuclear projection acquisition using phantom experiments. The motion compensation approach has been proposed for the setting of interventional imaging, in which external devices for the motion signal extraction are undesired and data-driven approaches are unstable due to the low count rates. With motion compensation, the 


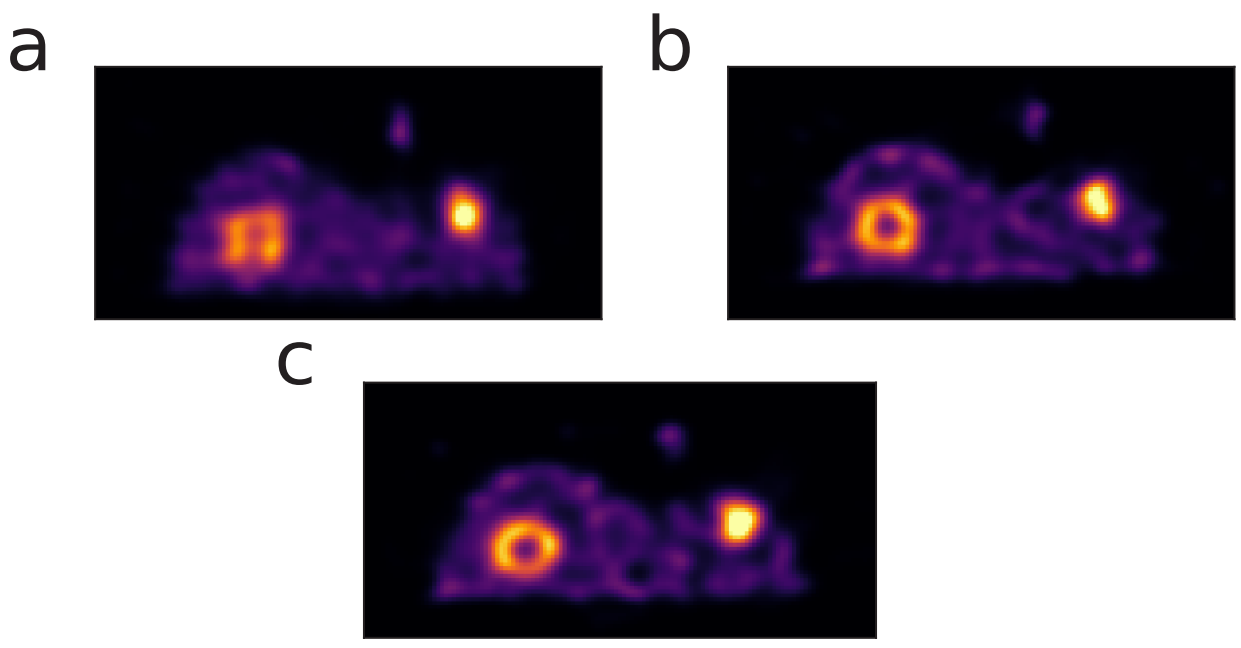

Figure 7: Slices of the SPECT reconstructions of the translating anthropomorphic phantom with the whole liver treatment configuration when made (a) without and (b) with motion compensation. The reconstruction obtained from the stationary anthropomorphic phantom is shown in (c) as a reference.

nuclear planar projections become easier to interpret and the quantitative accuracy of the SPECT reconstructions improves.

A limitation of this study was that the anthropomorphic phantom translated in only one direction and that the organ compartments did not change over the respiratory cycle. Real patient motion has translations in 3D and organ-specific translations and deformations. However, as long as these additional movements are stable over time, the motion compensation approach should provide similar results as described in this study seeing that the proposed method does not rely on any specific movement assumptions.

Since the flat panel detector is positioned in front of the gamma camera, the source-to-collimator distance is larger for IXSI than for a conventional SPECT/CT scanner. The improvements to the planar nuclear projections and the SPECT reconstructions by applying motion compensation are hence currently limited by the intrinsic spatial resolution of IXSI. When the flat panel detector thickness is reduced (e.g. by relocating the detector electronics), the intrinsic spatial resolution will be improved and the influence of motion compensation will increase.

The noise in the SPECT reconstructions is increased when performing motion compensation since the nuclear counts from only one motion bin are used. For the pre-treatment procedure of radioembolization (which is one of the proposed applications of IXSI), it has, however, been demonstrated that quantitative measures (e.g. the activity recoveries of the extrahepatic depositions) can be accurately determined even for high-noise reconstructions [12-14]. For interventional imaging, we hence believe it is more important to obtain quantitatively correct images rather than images with low noise. 
Table 2: The activity recoveries of the four spherical inserts (extrahepatic sphere 1 and 2 with 8.1 and $4.1 \mathrm{ml}$ volume respectively and the two intrahepatic spheres) obtained from the translating anthropomorphic phantom with the whole liver treatment configuration when reconstructed with and without motion compensations and from the stationary anthropomorphic phantom. The standard deviation was determined from the 10 individually analyzed noise realizations.

\begin{tabular}{l|ccc} 
& No compensation & Motion-compensated & No motion \\
\hline Extra - 1 & $36.8 \pm 0.8$ & $53.5 \pm 1.3$ & $51.9 \pm 1.1$ \\
Extra - & $50.6 \pm 1.2$ & $72.2 \pm 2.1$ & $71.9 \pm 1.8$ \\
Tumor - Necrosis & $41.7 \pm 0.4$ & $50.0 \pm 0.4$ & $49.8 \pm 0.9$ \\
Tumor - Solid & $53.5 \pm 0.4$ & $64.7 \pm 0.8$ & $65.2 \pm 0.4$
\end{tabular}

It might also be possible to extract a motion model from the motion-binned CBCT reconstructions so that it can be incorporated in the SPECT reconstruction. This approach has the advantage that no counts are disregarded and hence that the noise in the reconstruction is kept low. The approach was successfully demonstrated with digital simulations [15] but proved to be challenging due to the relatively low quality of the CBCT reconstruction when imaging at a low dose. Furthermore, the approach required several assumptions on the movement of the organs in the body, which may not necessarily hold in patients. Thus, although the performance of the motion-model approach will also be investigated when IXSI is employed in patient studies, the gating approach as in this study will be the primary objective.

The proposed motion compensation approach requires that the minimum and maximum motion amplitudes are relatively stable over the scan duration when performing SPECT/CBCT imaging since the motion signal is normalized to the peaks to account for the changing geometry and attenuation over the scanner rotation. The motion compensation performance hence decreases for motion patterns with substantial variations. This effect was previously studied with simulations [15] and was hence not further evaluated in this work.

A disadvantage of the proposed motion compensation approach is that an additional fluoroscopic dose is administered to the patient. However, when motion-compensated images are acquired with $60 \mathrm{kVp}$ beam strength for 15 minutes (e.g. planar imaging for $5 \mathrm{~min}$ and SPECT/CBCT for $10 \mathrm{~min}$ ), the total extra dose area product (DAP) that will be administered to the patient is only $0.8 \mathrm{mGy} \mathrm{m} \mathrm{m}^{2}$. By lowering the beam strength to $50 \mathrm{kVp}$, the extra administered dose will be even lower. As a comparison, the mean DAP that is already administered in our institute during the pre-treatment fluoroscopy procedure of radioembolization is $31.7 \mathrm{mGy} \mathrm{m}^{2}$ (not including the dose from the therapy itself). Hence, the extra dose administered by fluoroscopy-guided motion compensation is not expected to be a limiting factor for its clinical implementation.

\section{Conclusion}

Phantom experiments demonstrated that an interventional hybrid c-arm scanner, capable of simultaneous nuclear and fluoroscopic projection acquisition, can perform accurate compensation 
for respiratory motion at a low dose. Motion compensation improves the interpretation of the planar nuclear projections and increases the quantitative accuracy of the SPECT reconstructions. These improvements are expected to be beneficial for the guidance of interventional procedures involving radionuclides.

\section{References}

1. Braat AJAT, Smits MLJ, Braat MNGJA, et al. "Y90 Hepatic Radioembolization: An Update on Current Practice and Recent Developments”, J. Nucl. Med. 56(2) 1079-1087 (2015)

2. Vidal-Sicart S, Paredes P, Zanón G, Pahisa J, Martinez-Román S, Caparrós X, Vilalta A, Rull R, Pons F. Added value of intraoperative real-time imaging in searches for difficult-to-locate sentinel nodes. J. Nucl. Med. 51(8) 1219-25 (2010)

3. Van der Velden S, Kunnen B, Koppert WJC, et al. “A Dual-layer Detector for Simultaneous Fluoroscopic and Nuclear Imaging”, Radiology 290(3) 833-838 (2019)

4. Dietze MMA, Kunnen B, Van der Velden S, Steenbergen JHL, Koppert WJC, Viergever MA, De Jong HWAM, "Performance of a Dual-Layer Scanner for Hybrid SPECT/CBCT", Phys. Med. Biol. 64(10) 105020 (2019)

5. Bastiaannet R, Viergever MA, De Jong HWAM, "Impact of respiratory motion and acquisition settings on SPECT liver dosimetry for radioembolization”, Med. Phys. 44(10) 5270-5279 (2017)

6. McClelland JR, Hawkes DJ, Schaeffter T, King AP, "Respiratory motion models: a review”. Medical image analysis 17(1): 19-42 (2013)

7. Koppert WJC, Van der Velden S, Steenbergen JHL, De Jong HWAM, "Impact of intense x-ray pulses on a NaI (Tl)-based gamma camera”, Phys. Med. Biol. 63(6) 065006 (2018)

8. Koppert WJC, Dietze MMA, Van der Velden S, Steenbergen LJH, De Jong HWAM, "A comparative study of $\mathrm{NaI}(\mathrm{Tl}), \mathrm{CeBr}_{3}$, and $\mathrm{CZT}$ for use in a real-time simultaneous nuclear and fluoroscopic dual-layer detector", Phys. Med. Biol. 64(13) 135012 (2019)

9. Klein S, Staring M, Murphy K, Viergever MA, Pluim JP, "Elastix: a toolbox for intensity-based medical image registration", IEEE TMI 29(1) 196 (2010)

10. De Jong HWAM, Slijpen ETP, and Beekman FJ, "Acceleration of Monte Carlo SPECT simulation using convolution-based forced detection”, IEEE Trans. Nucl. Sci. 48(1) 58-64 (2001)

11. Dietze MMA, Van der Velden S, Lam MGEH, Viergever MA, De Jong HWAM, "Fast quantitative reconstruction with focusing collimators for liver SPECT”, EJNMMI Phys. 5(1) 28 (2018)

12. Van der Velden S, Dietze MMA, Viergever MA, De Jong HWAM, "Fast technetium-99m liver SPECT for evaluation of the pretreatment procedure for radioembolization dosimetry", Med. Phys. 46(1) 345$355(2019)$

13. Kunnen B, Dietze MMA, Braat AJAT, Lam MGEH, Viergever MA, de Jong HWAM, "Feasibility of imaging ${ }^{90} \mathrm{Y}$ microspheres at diagnostic activity levels for hepatic radioembolization treatment planning", Med. Phys. 47(3) 1105-1114(2019)

14. Dietze MMA, Kunnen B, Beijst C, de Jong HWAM, "Adaptive scan duration in SPECT: Evaluation for radioembolization”, Med. Phys. 47(5) 2128-2138 (2020)

15. Dietze MMA, Bastiaannet R, Kunnen B, Van der Velden S, Lam MGEH, Viergever MA, De Jong HWAM, "Respiratory motion compensation in interventional liver SPECT using simultaneous fluoroscopic and nuclear imaging”, Med. Phys. 46(8) 3496-3507 (2019) 


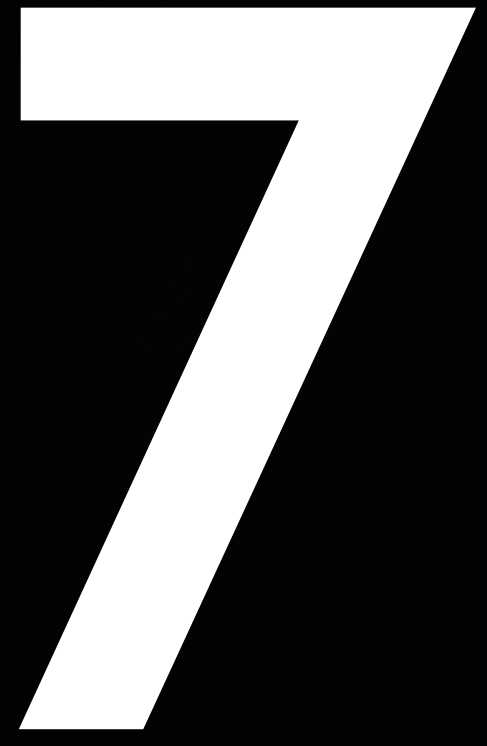




\section{NUCLEAR IMAGING WITH FOCUSING COLLIMATORS}

PUBLISHED AS:

MMA Dietze, S van der Velden, MGEH Lam, MA Viergever, HWAM de Jong

Fast quantitative reconstruction with focusing collimators for liver SPECT

EJNNMI Physics 5(1):28 (2018) 


\begin{abstract}
Background

The generation of a SPECT scan during the procedure may aid in the optimization of treatments as liver radioembolization by offering image-guided dosimetry. This, however, requires both shortened acquisition times and fast quantitative reconstruction. Focusing collimators increase sensitivity and thus may speed up imaging. Monte Carlo-based iterative reconstruction has shown to provide quantitative results for parallel hole collimators, but may be slow. The purpose of this work is to develop fast Monte Carlo-based reconstruction for focusing collimators and to evaluate the impact of reconstruction and collimator choice on quantitative accuracy of liver dosimetry by means of simulations.
\end{abstract}

\title{
Results
}

The developed fast Monte Carlo simulator was found to accurately generate projections compared to a full Monte Carlo simulation, providing projections in several seconds instead of several days. Monte Carlo-based scatter correction was superior to other scatter correction methods in describing recovered activity and reached similar noise levels as dual-energy window scatter correction. Although truncation artifacts were present in the cone beam collimator $(50 \mathrm{~cm})$, the region inside the field of view (FOV) could be reconstructed without loss of accuracy. Provided the object to image is inside the FOV, the focusing collimator with $50 \mathrm{~cm}$ focal distance could retrieve the same noise levels as a parallel hole collimator in $68 \%$ of the total scanning time, the multifocal collimator in $73 \%$ of the time, and the $100 \mathrm{~cm}$ focal distance collimator in $84 \%$ of the time.

\section{Conclusion}

Focusing collimators combined with Monte Carlo-based reconstruction have the ability to enable quantitative imaging of the FOV in a significantly shorter timeframe. The proposed approach to the forward projector will additionally make it possible to reconstruct within minutes. These are crucial steps in moving toward real-time dosimetry during interventions. 


\section{Background}

Liver malignancies are increasingly treated with intra-arterial microsphere radioembolization $[1,2]$. In this procedure, beta-emitting radioactive isotopes as yttrium-90 $\left({ }^{90} \mathrm{Y}\right.$ ) (encapsulated by either glass (TheraSphere; MDS Nordion, Ontario, Canada) or resin (SIR-Spheres; Sirtex Medical, Sydney, Australia)), holmium-166 ( ${ }^{166} \mathrm{Ho}$ ) (QuiremSpheres; Quirem, Deventer, The Netherlands) are injected into the hepatic artery. The microspheres distribute according to the vascularity, ideally occluding small tumor capillaries in which they deliver a high dose to the tumor whilst sparing the healthy parenchyma.

Single photon emission computed tomography (SPECT) of technetium-99m macroaggregated albumin ( $\left.{ }^{99 \mathrm{~m}} \mathrm{Tc}-\mathrm{MAA}\right)$ is normally performed before treatment to study the sphere deposition in the liver and to ascertain minimal dose to healthy liver parenchyma and lungs. Ideally, this scout scan would be performed shortly before the treatment to minimize changes in pathology and catheter position. In addition, if the scout scan proves affirmative, it can be directly followed by the treatment in a single procedure. Such image-guided procedures are, however, not common practice because SPECT/CT is not available in the intervention room and the combined time of transporting the patient to the scanning room and performing a SPECT scan is too long to hold the intervention room in most hospitals [3]. We envision a gamma camera in the intervention room to eliminate the need for patient transportation. Additionally, if such a device were to acquire accurate SPECT images in a matter of minutes, real-time image-guided radioembolization procedures would become feasible.

To reduce the SPECT scanning times, we propose to use focusing collimators to increase sensitivity. Historically, this has been done in brain SPECT using a cone beam collimator [4,5], inasmuch as the object to reconstruct is smaller than the size of the gamma camera. More recently, a multifocal collimator (e.g. SmartZOOM on the IQ-SPECT) for cardiac SPECT has been introduced in clinical practice [6,7], with the aim of improving sensitivity in the area of interest, whilst capturing the unfocused photons to reduce truncation artifacts (see Fig. 1). Such collimators could function in radioembolization SPECT similarly, since the volume of dosimetric interest is often located in a predetermined region (e.g., retrieved from a CT- or MRI-scan).

For image-guided procedures to be successful, not only the acquisition stage should be fast, but quantitative reconstruction should also be performed in a matter of minutes. To ensure that image quality is preserved whilst reducing reconstruction time, sophisticated reconstruction algorithms can be used. This study will make use of Monte Carlo-based iterative reconstruction, which has previously been shown to improve quantitative results for several isotopes and parallel hole collimators [8-10]. Focusing collimators for these reconstructions introduce new characteristics, such as a shift-variant point spread function (PSF) and potentially severe truncation artifacts. The purpose of this work is to evaluate to what extent the combination of Monte Carlo-based iterative reconstructions and focusing collimators can realize the need for fast and quantitatively accurate liver SPECT imaging in the intervention room, by means of a simulation study. 

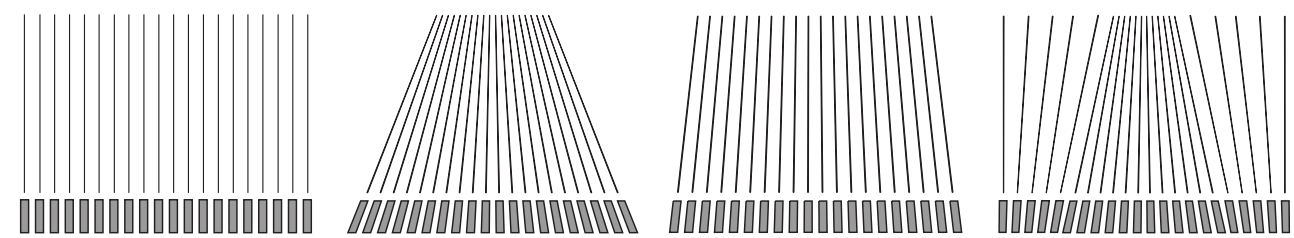

Figure 1: Illustration of the field of view (FOV) of the discussed collimators. From left to right: parallel hole collimator, cone beam collimator $(50 \mathrm{~cm})$, cone beam collimator $(100 \mathrm{~cm})$, multifocal collimator.

\section{Methods}

\section{Simulated collimators}

Four specific configurations were considered for the comparison of focusing collimators: the regular parallel hole collimator, cone beam collimators with focal lengths of $50 \mathrm{~cm}$ and $100 \mathrm{~cm}$, and a multifocal collimator [11] (Fig. 1). These collimators were chosen as they are often already available at hospitals (or can be readily made) for the previously mentioned procedures of brain and cardiac imaging.

To be able to make accurate comparisons, the collimator parameters were standardized: all collimators were equipped with LEHR specifications $(24.05 \mathrm{~mm}$ hole length, $1.11 \mathrm{~mm}$ hole diameter, $0.16 \mathrm{~mm}$ septal thickness), as is customary for ${ }^{99 \mathrm{~m}} \mathrm{Tc}$ imaging [12], and covered a camera crystal surface of $53.3 \times 38.7 \mathrm{~cm}^{2}$ (Siemens Symbia T characteristics [12]). The multifocal collimator was equipped with a focal distance of $50 \mathrm{~cm}$ and a focusing area covering half of the total surface: $26.7 \times 19.4 \mathrm{~cm}^{2}$.

To illustrate the effect of the varying field-of-views of the different collimators with respect to liver coverage, the XCAT phantom [13] is shown together with the four collimators rotating at a distance of $1.0 \mathrm{~cm}$ from the body in Fig. 2. The parallel hole collimator has no spatial dependence and has thus equal information over the entire liver. The cone beam collimator $(50 \mathrm{~cm})$ has complete information of only part of the liver, whilst the cone beam collimator $(100 \mathrm{~cm})$ is able to encapsulate the entire organ. The multifocal collimator images part of the liver with increased sensitivity, and the remainder with decreased sensitivity. Notably different from the usual cardiac imaging is that not one sharply defined sphere with increased sensitivity is imaged, but, since the liver lies more offcenter in the body than the heart, this volume is smeared out over a larger volume.

The cone beam collimators may be prone to truncation artifacts. When a large fraction of activity is present outside the area that is imaged in most projections, this could result in incorrect quantification. The multifocal collimator has a FOV equal to that of a parallel hole collimator and hence does not have this problem. However, this geometry results in a decrease in sensitivity in the background, which might reduce the advantage of the focusing geometry. The magnitude of these effects was investigated using a Monte Carlo simulation study. 


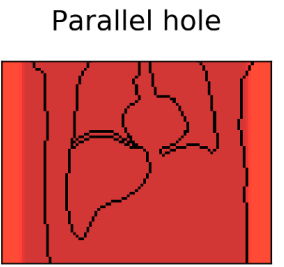

Cone beam $(50 \mathrm{~cm})$
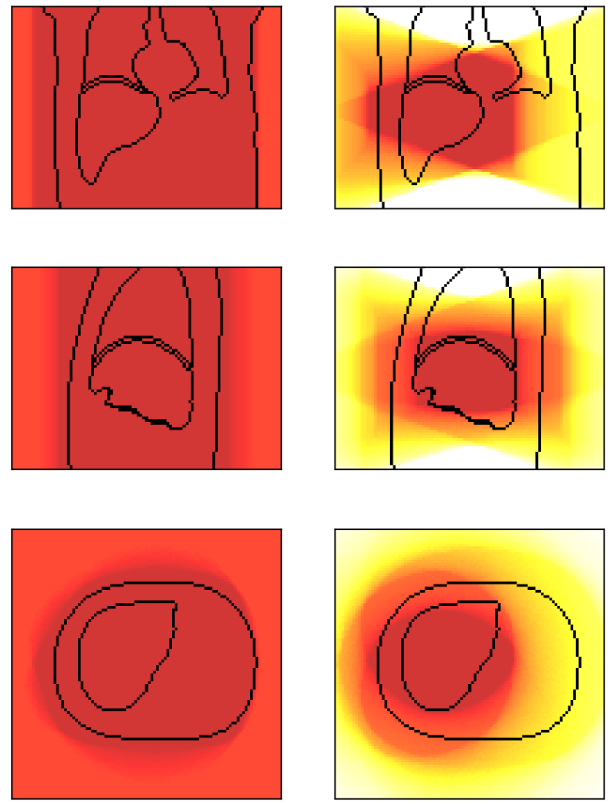

Lowest sensitivity
Cone beam $(100 \mathrm{~cm})$
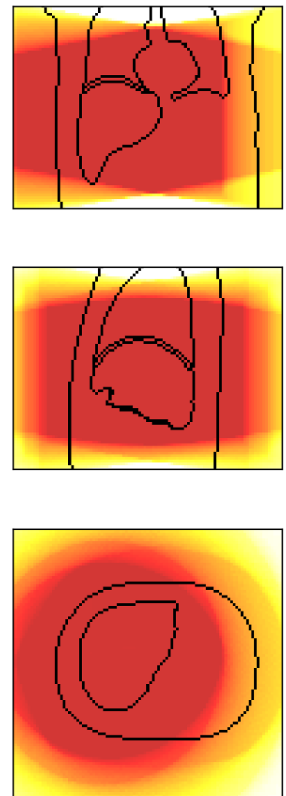

Highest sensitivity
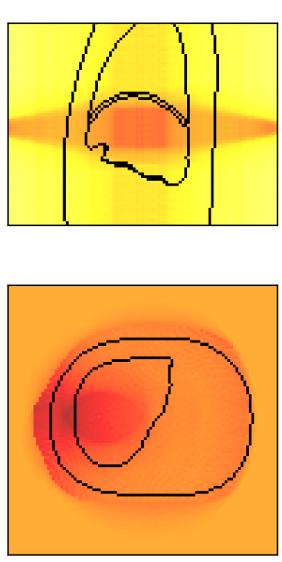

Multifocal

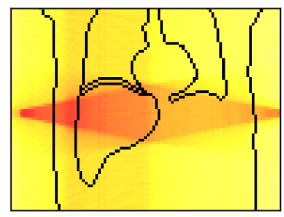

Figure 2: From top to bottom are the coronal, sagittal and transverse planes of the XCAT phantom (drawn are the body, liver, and lung contours) for the simulated collimators. The color gives an indication of the geometric sensitivity of each collimator. The detector surface follows a closed (non-circular) contour of the patient whilst rotating around the center of the liver.

\section{Point Spread Function modeling}

The Utrecht Monte Carlo System (UMCS) is a Monte Carlo-based iterative SPECT reconstruction package. UMCS is able to simulate photon interactions inside the body in a fast manner, so that implementation in the clinic is feasible. The forward projector has previously been validated for ${ }^{99 \mathrm{~m}} \mathrm{Tc},{ }^{90} \mathrm{Y}$, and ${ }^{166} \mathrm{Ho}$; a complete description can be found elsewhere [8-10]. UMCS applies convolution-based forced detection (CFD), which projects a point spread function (PSF) from every source or scatter position to the camera. To implement CFD for focusing collimators, the object transformation was performed using rotating and warping in a single interpolation step [14]. Additionally, several characteristics of focusing collimators differ from those of regular parallel-hole collimators and affect the shape and magnitude of the PSF (see Fig. 3):

1. The distance of a point source to the collimator surface increases with spatial distance from the middle of the collimator, broadening the PSF.

2. There is a change in coordinate system as the collimator captures photons over a sphere, while the detector is flat. This introduces a Jacobian determinant of $1 / \cos (\theta)^{2}$ (with $\theta$ being the angle of the irradiated collimator hole) [14] and thus induces spatial dependence on gamma camera sensitivity. 


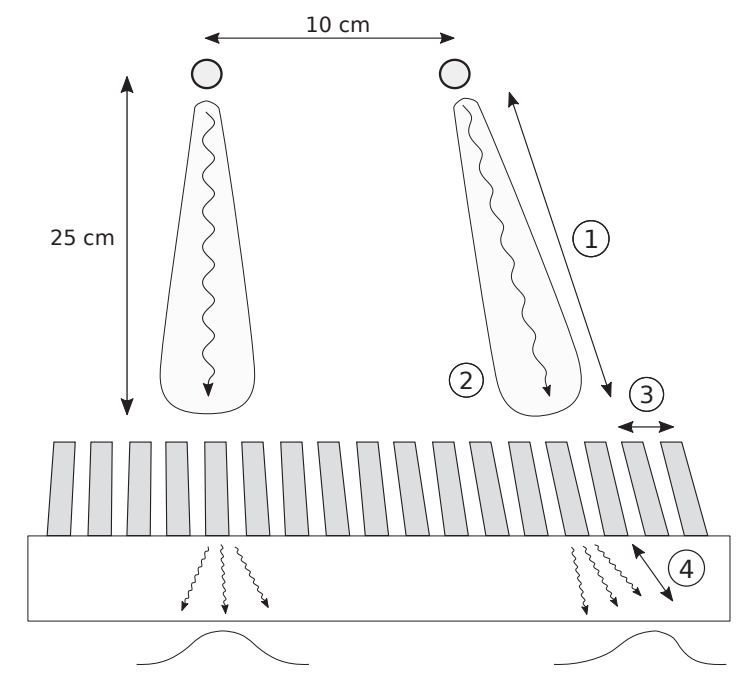

Figure 3: Differences between a point source located in the middle of a focusing collimator, versus one located toward the side. The effects corresponding to the numbers can be found in the text. It can be expected that the PSF of a point source located in the origin has a different shape than a point source toward the edge; the severity of this effect was studied in a simulation with point sources as located in the figure.

3. Hole diameter and septa thickness increase with distance from the origin, changing the number of photons that interact with the collimator.

4. The attenuation of photons in the crystal increases with distance from the origin. Since photons enter the crystal under an angle, this makes the shape of the PSF slightly asymmetric.

To study the severity of the above effects on the shape of the PSF, a simulation study was performed on the cone beam $(50 \mathrm{~cm})$ collimator. The collimator configuration was modeled in GATE [15] with one point source positioned at the center of the collimator and one at the edge of the collimator (with a spatial shift of $10 \mathrm{~cm}$ ), both at a distance of $25 \mathrm{~cm}$ from the gamma camera (see Fig. 3). This is a region in which the greatest deviation between PSFs is expected, because the offset point source is detected close to the edge of the gamma camera. The differences in magnitude and shape of the retrieved PSFs were measured for comparison.

\section{Acceleration of shift-variant PSF modeling}

As mentioned, the shift variance of the PSF will be greatest for regions toward the side with a large distance from the detector. For the other regions, the effect will likely be not significant in comparison to the intrinsic detector resolution. We propose that it is sufficient to perform only a single convolution per focusing/diverging region to accurately describe the detector physics, thus only accounting for the Jacobian determinant. The shift variance of the PSF was thus not included in the reconstruction. This may slightly reduce the accuracy, but will greatly improve the reconstruction speed. 


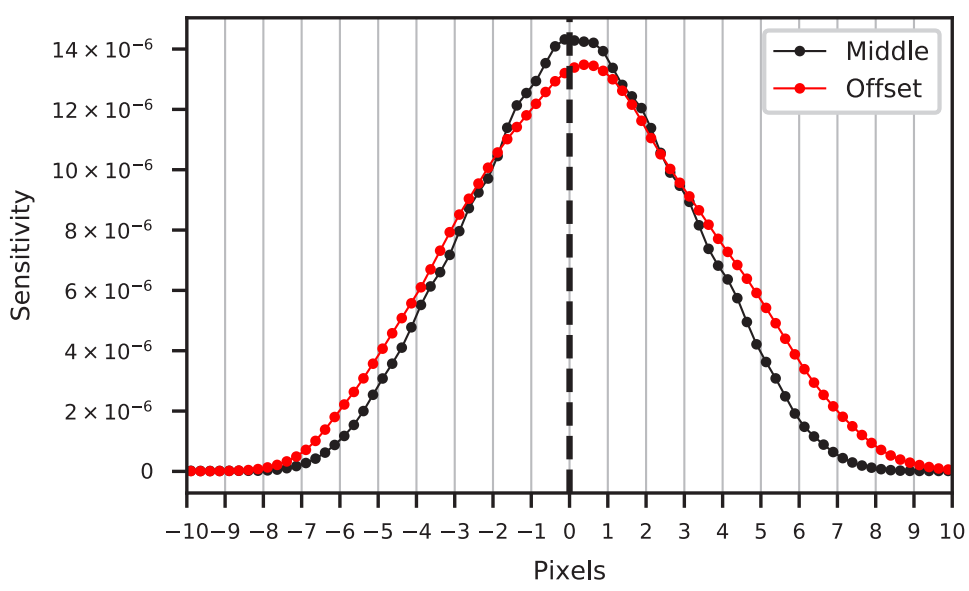

Figure 4: Difference in Point Spread Function for a point source located in the origin at $\mathrm{z}=25 \mathrm{~cm}$ and one with an offset of $\mathrm{dx}=10 \mathrm{~cm}$. These PSFs were corrected for the $1 / \cos (\theta)^{2}$ sensitivity factor.

Thus, for the cone beam collimators, one convolution per step in height is required over the $\mathrm{x} / \mathrm{y}$ plane to model the detector physics; for the multifocal collimator, four regions are defined: one with focusing in both directions, one with focusing in $\mathrm{x}$ and divergence in $\mathrm{y}$, one with divergence in $\mathrm{x}$ and focusing $\mathrm{y}$, and finally one with divergence in both directions. The regions with combined focusing and divergence have an elliptical shape and thus no rotational averaging was performed. The border where the different regions meet was averaged over a width of two pixels.

\section{Projector evaluation}

To evaluate the performance of the proposed fast forward projector, projections of an image quality phantom (IEC NEMA 2007; PTW, Freiburg, Germany) were compared against full Monte Carlo simulation in GATE for all collimator configurations. The spheres of the digital NEMA phantom were configured with a 10:1 background ratio of ${ }^{99 \mathrm{~m}} \mathrm{Tc}$ and photons were generated until the smallest sphere was visually distinguishable from the background. The profiles of the spheres in the projections were compared to verify whether the PSF was indeed correctly modeled and whether the sensitivity of the system was accurate. The speed of both simulators was compared to find the acceleration factor of the proposed fast Monte Carlo projector over the full one.

\section{Digital phantom study}

To evaluate the performance of the mentioned collimators for quantitative liver SPECT, reconstructions of the XCAT liver and lung were made. A tumor (radius of $20 \mathrm{~mm}$; positioned in the converging region of all collimators) was inserted into the liver at a 5:1 uptake ratio. The total activity in the phantom was $100 \mathrm{MBq}$. The lung was filled uniformly with a lung shunt fraction (LSF) of 5\%. To speed up the simulations, the fast forward projector of UMCS was used to create projections, on which Poisson noise was added to emulate a clinical setting. 


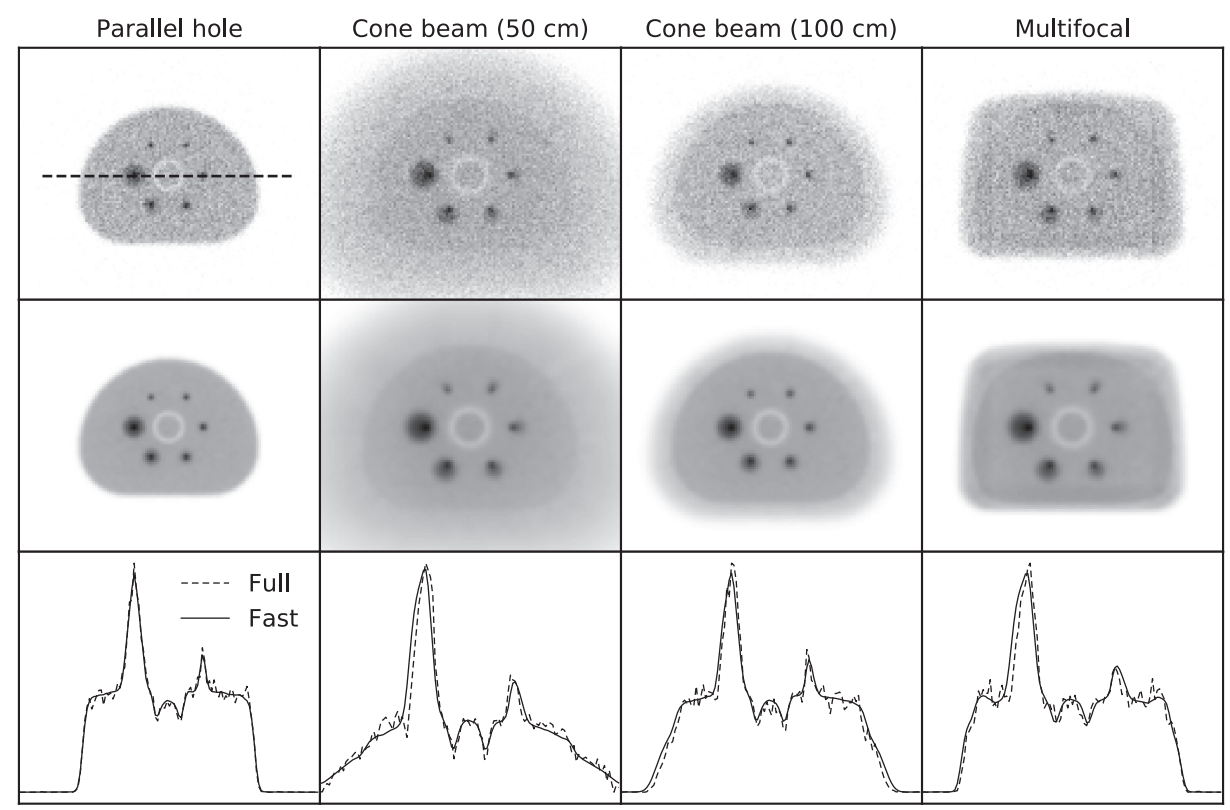

Figure 5: Projections of the NEMA phantom made with the GATE Monte Carlo projector (top row) and with the fast Monte Carlo projector (middle row) for the parallel hole, cone beam $(50 \mathrm{~cm})$, cone beam $(100 \mathrm{~cm})$, and multifocal collimators. Profiles along the middle spheres are shown in the bottom row. The GATE Monte Carlo projections are generated with $2.6,4.1,2.7,2.6$ billion photons, respectively.

The obtained projections were subsequently reconstructed with attenuation and scatter correction and resolution recovery (PSF modeling) in both forward and backward projections, with eight subsets for ten iterations. The photon physics were simulated based on the phantom density map in the forward projection. Ten noise realizations were performed to retrieve an indication of the stability of the reconstructions. The phantoms were configured in a $128 \times 128 \times 90$ grid, with 4.7 $\mathrm{mm}$ isotropic voxels. The energy window was set at $15 \%$ around the $140 \mathrm{keV}$ photopeak.

The effect of truncation on the reconstruction was evaluated by reconstructing the liver phantom with and without physical camera size; the latter assumes an infinitely large detector surface. The reconstructed activity in the tumor was measured to study whether truncation affects the quantitative results inside the FOV.

For comparison of the Monte Carlo-based scatter correction, reconstructions were also performed with a dual-energy window scatter correction [16] and without scatter correction. In the case of dual-energy window scatter correction, projections of the scatter window (20\% around $110 \mathrm{keV}$ ) were additionally simulated - again with Poisson noise added. These scatter projections were smoothed by a Gaussian filter of two pixels, added to the reconstruction loop and weighted with a $k$ factor for scaling of the difference of scatter in the photopeak window and the scatter window, which was evaluated at the clinically used value of $k=0.5$ and an optimal value. This optimal $k$-value was first based on the ratio of the scatter in the photopeak and the scatter energy window, and 


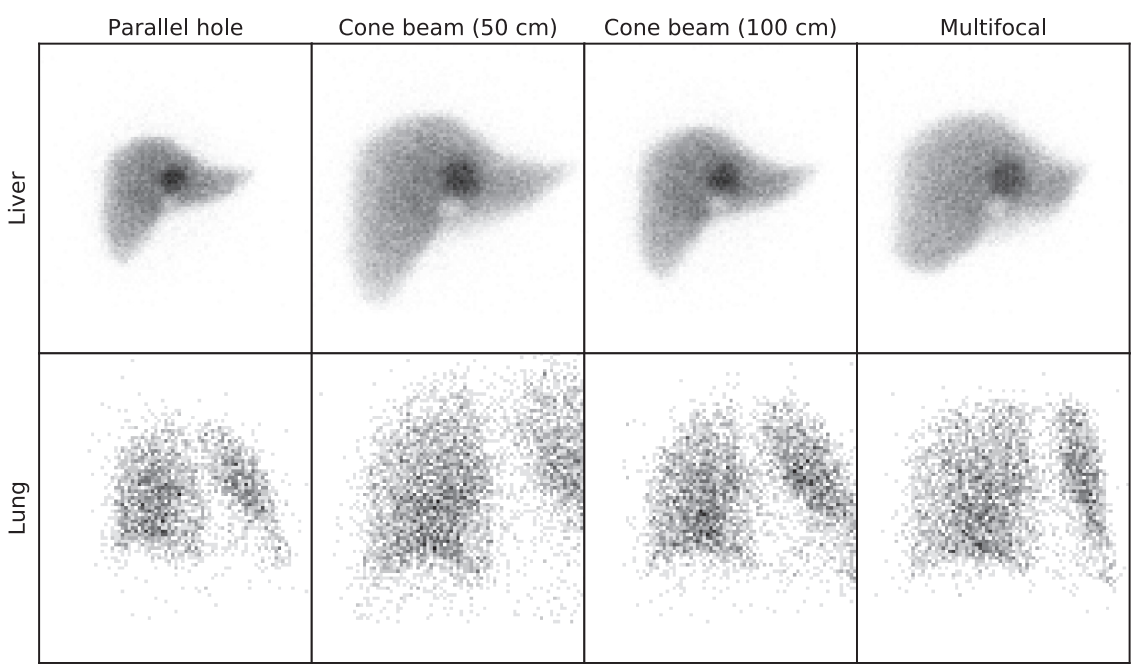

Figure 6: Projections of the XCAT liver and lung made with the fast Monte Carlo projector, with Poisson noise added to emulate a detector measurement of 20 seconds. The number of counts in the liver projections is $56 \mathrm{k}$, $104 \mathrm{k}, 75 \mathrm{k}$, and $90 \mathrm{k}$, respectively. For the lung, these are 3458, 6376, 5406 and 5144, respectively.

was then further tuned by additionally simulating more $k$-values both above and below this first estimate. The $k$-value resulting in the lowest mean squared error with the phantom over the entire liver was used as the optimal value. The recovery profiles of the different scatter corrections methods were measured to study their effect on tumor contrast.

Subsequently, the noise properties of the collimators and scatter correction methods were studied in more detail. To evaluate whether they are able to reduce scanning times, reconstructions were performed for several view durations $(20 \mathrm{~s}$ - as is clinical protocol, $17.5 \mathrm{~s}, 15 \mathrm{~s}, 12.5 \mathrm{~s}, 10 \mathrm{~s}, 7.5 \mathrm{~s}$ and $5 \mathrm{~s}$ ). A tumor VOI was created by selecting the tumor region and eroding it with one pixel to reduce partial volume effects. As the total reconstruction quality is of primary interest, the spatial noise dependence was not studied. Therefore, the background liver VOI, for which no truncation artifacts were present, was created by selecting the hot region for the cone beam $(50 \mathrm{~cm})$ collimator from Fig. 2 and subtracting the tumor VOI. The lung VOI was created similarly, except that no tumor VOI had to be subtracted.

\section{Results}

\section{Point Spread Function modeling}

The profiles of the simulated point sources in the origin and at the edge of the cone beam collimator $(50 \mathrm{~cm})$, blurred with the intrinsic gamma camera resolution of $3.8 \mathrm{~mm}$ full-width at half-maximum (FWHM) [12], are shown in Fig. 4. It can be seen that the difference between the means of the distributions is less than one pixel $(4.7 \mathrm{~mm})$. This means that, other than the $1 / \cos (\theta)^{2} \mathrm{Jacobean}$ factor introduced by the coordinate change, the shape and sensitivity of the PSF only changed slightly. 

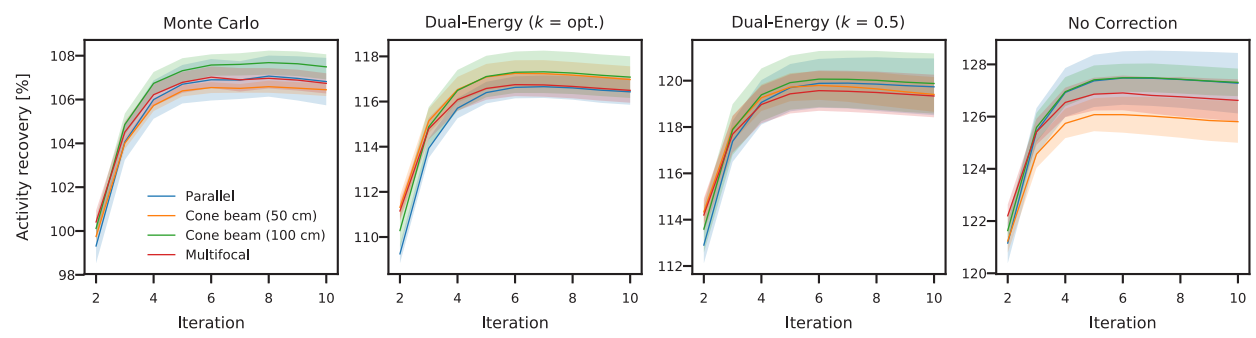

Figure 7: Activity recovery in the tumor, collected per scatter correction method. The shaded bars indicate the standard deviation retrieved from the ten noise realizations.

\section{Projector evaluation}

The projections of the NEMA phantom simulated with the fast Monte Carlo simulator (UMCS) and the full Monte Carlo simulator (GATE) are shown in Fig. 5. The profiles over the middle spheres are visualized and agree well with each other. Hence, the proposal of a single convolution per focusing region is sufficient and UMCS can be used as a quantitative forward projector for focusing collimators.

The fast forward projector was able to generate a projection in approximately 2 seconds, whilst the full Monte Carlo projection took 4 days (single-threaded) to obtain images with a quality as in Fig. 5 on a regular desktop PC. The total reconstruction time for ten iterations with the mentioned matrix configuration using the fast forward projector was approximately one hour.

\section{Digital phantom study}

Projections of the XCAT lung and liver (with added Poisson noise) can be found in Fig. 6. The number of counts for the focusing collimators was higher than for the parallel hole geometry. Since the resolution of all collimators is equal, more accurate reconstruction results should therefore be possible with the focusing collimators.

The activity recovery (activity of the reconstruction divided by activity of the phantom) in the tumor with the various collimators is presented in Fig. 7. Convergence rate is approximately the same for all collimators. However, absolute activity recovery values differ for the various methods of scatter correction: approximately $106 \%$ is retrieved for the Monte Carlo-based method, $116 \%$ for the dual-energy window method with optimal $k, 119 \%$ with clinical $k=0.5$, and $127 \%$ for no scatter correction applied. As the activity recovery was found to be converged after ten iterations, this setting was used in the remaining analysis.

Reconstructions of the digital phantoms are displayed in Fig. 8, shown separately for reconstructions of 5 and 20 seconds per view. It can be observed that the background noise was reduced in the reconstructions of the focusing collimators. The lung reconstructions possessed higher noise levels than those of the liver, due to their lower activity. It is furthermore evident how truncation influenced the results, inasmuch as in the cone beam $(50 \mathrm{~cm})$ reconstruction streak artifacts appeared toward the edge of the phantoms. The objects within the FOV, however, seem to be reconstructed correctly for all collimators. 

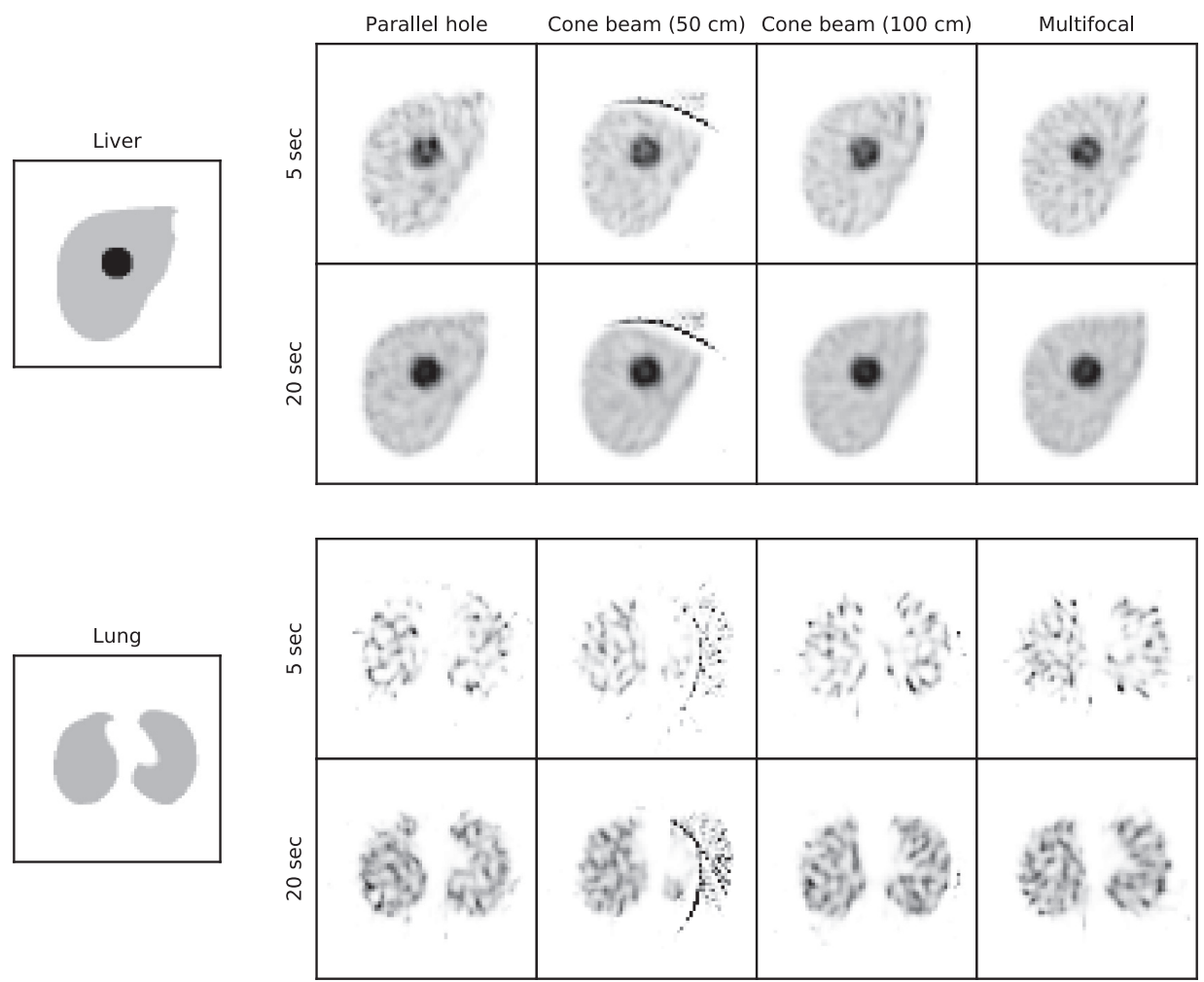

Figure 8: Reconstructions of the XCAT liver and lung for all collimators for 5 and 20 seconds per view reconstructed with Monte Carlo-based scatter correction.

\section{Truncation}

To study the effect of truncation, the activity recovery in the tumor of the liver reconstructions with and without truncation was compared, for the cone beam $(50 \mathrm{~cm})$ collimator; see Fig. 9 . The reconstructions with truncation converge toward the same value as those without truncation. Hence, truncation artifacts do not significantly influence the quantitative accuracy inside the FOV for this configuration. The volume where no artifacts were present (i.e., the volume seen by more than half of the total number of projections) was found to be $86 \%$ of the liver and $51 \%$ of the lung total volume.

\section{Scatter correction}

To study the effect of scatter correction, activity recovery profiles of the various scatter correction techniques were generated; these are shown in Fig. 10. It is evident that reconstruction without scatter correction leads to an overestimation of activity over the entire profile. Dual-energy window scatter correction was able to trace the contours better, since the scattered photons are added to the reconstruction loop. Dual-energy window scatter correction with optimal $k$ factor (found to be $k$ $\sim 0.72$ for all collimators) performed better than with clinical $k=0.5$ for this phantom, especially 

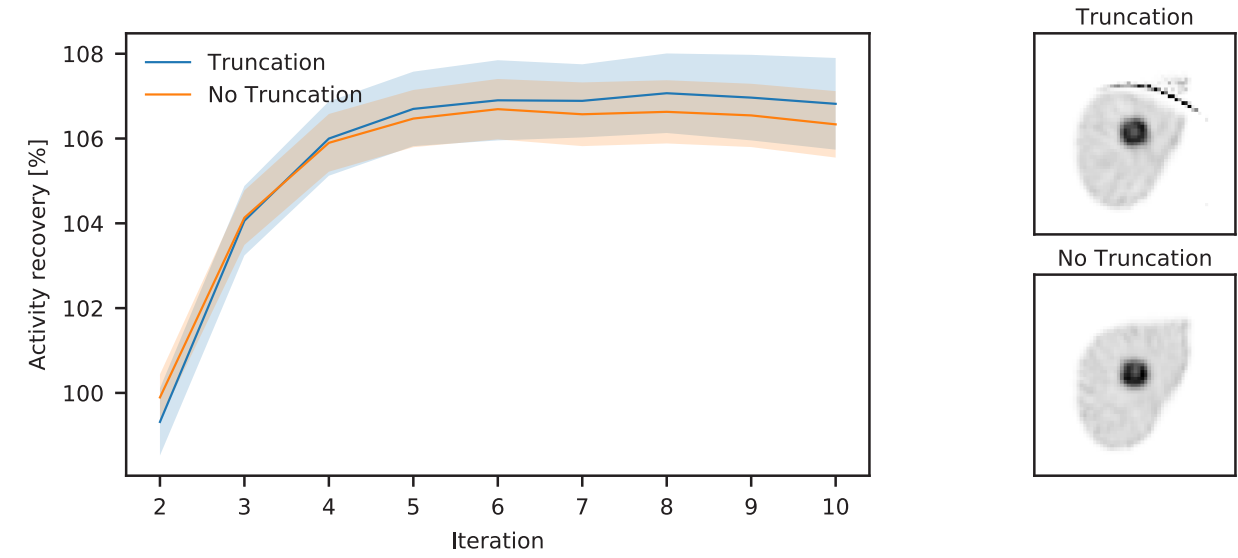

Figure 9: Activity recovery of reconstructions with and without truncation, for the cone beam $(50 \mathrm{~cm})$ collimator reconstructed with Monte Carlo-based scatter correction.

in the background region. Both options however have difficulty with correct quantification of the tumor. Monte Carlo-based scatter correction was able to trace the contours most accurately, both in the background and around the tumor.

These results were further detailed by calculating for each of the collimators the absolute difference with the ground truth (phantom), shown in Fig. 11 separately for the tumor and the background VOI of the liver phantom. It can again be observed that Monte Carlo-based scatter correction outperforms the other options.

\section{Scan time reduction}

To study the effect of scanning times on the reconstructions, projections of the digital phantoms were simulated for several view durations. It can be seen in Fig. 12 that the focusing collimators yielded a reduced noise level in comparison to the parallel hole collimator. The trends in the liver and the lung phantom were comparable.

The total possible time gain of the focusing collimators can be found by calculating the points on the curves where a similar noise level is achieved as with the parallel hole collimator at 20 seconds per view (clinical setting). This shows that comparable quality can be achieved in approximately $13.5 \mathrm{~s}$ per view for the cone beam $(50 \mathrm{~cm}$ ) collimator (thus $68 \%$ of the total scanning time), $14.5 \mathrm{~s}$ per view ( $73 \%$ of the scanning time) for the multifocal collimator, and $16.7 \mathrm{~s}$ per view ( $84 \%$ of the scanning time) for cone beam $(100 \mathrm{~cm})$ collimator.

Similarly to the noise properties of the focusing collimators with respect to the parallel hole collimator, the effects in scatter correction method can be evaluated. These results are presented in Fig. 13 for all considered collimators. No scatter correction has the lowest level, as no corrections are performed for this option. Monte Carlo-based and dual-energy window scatter correction can be found to achieve comparable noise levels. 


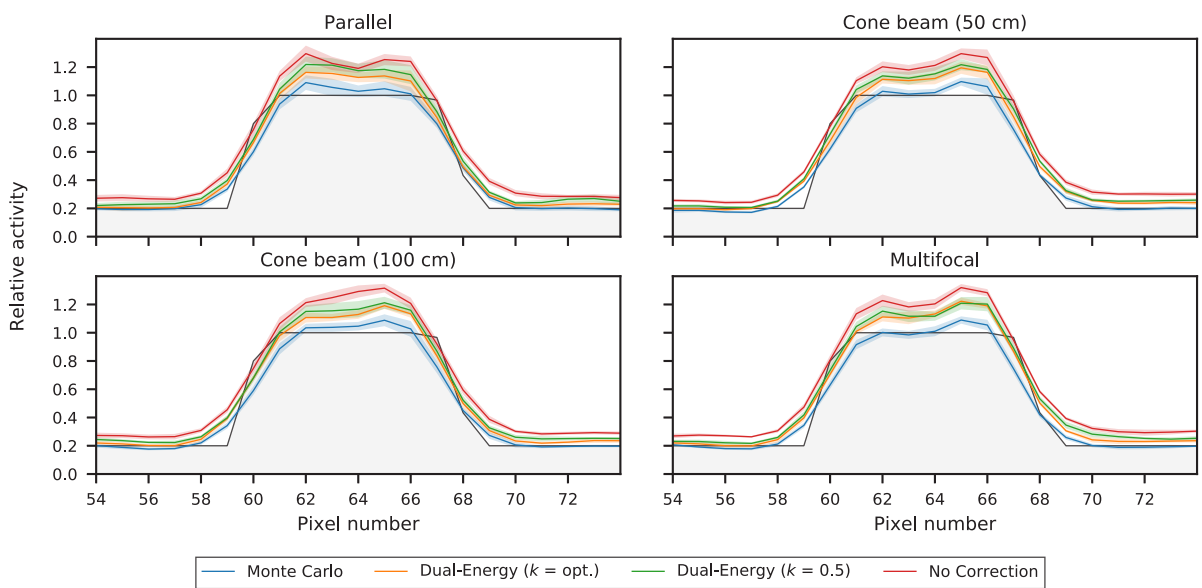

Figure 10: Activity recovery profiles, normalized to the maximum activity, of the investigated scatter correction techniques for the several collimators. In black is the phantom profile.

\section{Discussion}

This study investigated to what extent quantitative liver SPECT imaging could be accelerated by combining focusing collimators with Monte Carlo-based reconstruction. We showed that focusing collimators are able to reduce scanning times in comparison to the clinical procedure - provided that the object to image is inside the FOV. The proposed approach to the forward projector allows fast reconstruction and gives quantitative results. This is an important step in realizing real-time imageguided radioembolization procedures.

No definitive conclusion was reached in this work as to which collimator is best suited for liver SPECT, since this strongly depends on liver size and tumor locations. We therefore consider this an exploratory study in which the implementation of fast reconstruction of focusing collimators is discussed and the individual effects of truncation and scatter are shown. In the case of radioembolization, it is desired to have homogeneous image quality over the entire liver. This would favor the cone beam $(100 \mathrm{~cm})$ or multifocal collimator. However, in the case of a solitary tumor, the cone beam $(50 \mathrm{~cm})$ collimator would be more beneficial.

The SPECT/CT scans required for radioembolization do not necessarily have to be performed with 20 seconds per view. It may be that reconstructions gathered with a shorter time per view are also of sufficient quality for clinical use. However, also for these faster scans, there will be a benefit in using focusing collimators. For a 10 minute scan e.g. one could move toward $\sim 7$ minute scanning time. With the aim of interventional scanning as described in this work, the gain of a few minutes in scanning time could be crucial to facilitate implementation in the clinic.

Other than the localization of activity distributions, one clinical use of the ${ }^{99 \mathrm{~m}} \mathrm{Tc}$ scout dose is the determination of the LSF. As discussed, the FOV of the focusing collimators is smaller than that of the parallel hole collimator, which therefore potentially requires multiple separate acquisitions to 

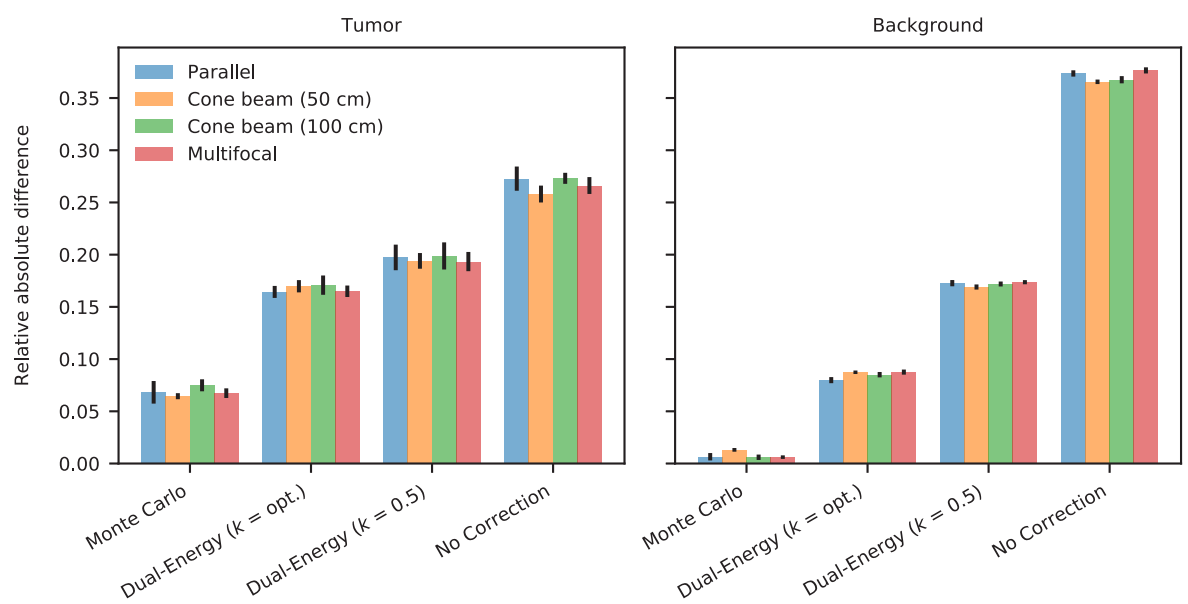

Figure 11: The relative absolute difference, normalized to the mean activity, of the reconstructions with the phantom (ground truth), shown separately for the tumor and the background region, for the four collimators.

also cover the lung region. However, as the LSF is determined over two large regions, it is much less sensitive to low count rates. It is envisioned that one or two acquisitions can thus be quickly (likely $<1$ minute) performed to calculate the LSF. This LSF procedure will thus likely not be limiting for the real-time image-guided procedures.

This work has studied ${ }^{99 \mathrm{~m}} \mathrm{Tc}$ for use as scout dose to facilitate fast implementation in the clinic. Promising research is however ongoing on the use of ${ }^{166} \mathrm{Ho}[17,18]$ as a scout dose for the corresponding liver radioembolization, so that scout and treatment particle are equal. This will be investigated for ${ }^{90} \mathrm{Y}$ similarly. For these isotopes, bremsstrahlung will be the most important measure and scatter modeling will have an even larger impact than for ${ }^{99 \mathrm{~m}} \mathrm{Tc}$ imaging. It will be straightforward to extend this simulation study to the above-mentioned isotopes by generation of new point spread functions.

Focusing collimators are not the only option for scan time reduction. Other options are, for instance, gating of the respiratory signal [19], so that motion artifacts are suppressed, and optimization of the time spent per view [20], so that more counts are collected in the projections that carry most information. Ideally, all these options would be combined in a single system.

SPECT reconstruction with Monte Carlo-based scatter correction comprising ten iterations with clinical parameter settings with our implementation currently takes approximately one hour on a single-threaded regular desktop. Obviously, this is too long for inclusion into an image-guided procedure. The most time-consuming step in this reconstruction is the rotating and warping transform, due to the many memory look-up operations required. If this step is optimized and implemented on a GPU-based workstation, we expect that reconstruction in the order of minutes is achievable. 

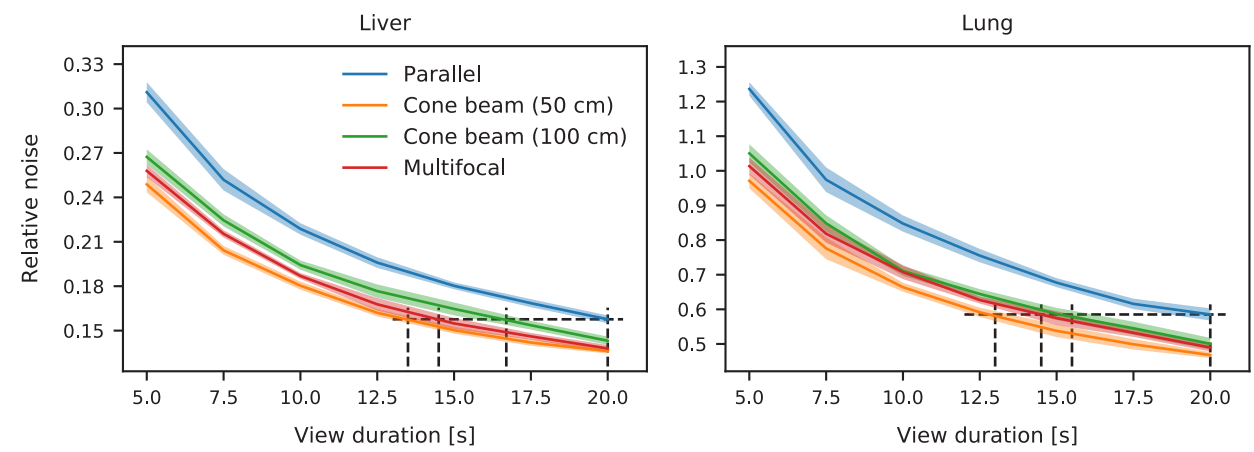

Figure 12: Noise levels, normalized to the mean activity, for the four collimators obtained by reconstruction with Monte Carlo-based scatter correction. The dotted framework shows the time needed for the conebeam and multifocal collimators to achieve the same image quality as the parallel hole collimator at the clinical setting of 20 seconds per view.

Commercial reconstruction software is generally already able to retrieve reconstructions within a few minutes, thus too facilitating real-time image-guided procedures. These packages however usually employ dual-energy window scatter correction, of which it was shown that quantification fails for smaller distributions. For such distributions, the more extensive Monte Carlo-based scatter correction would be better suited to accurately describe the true activity.

In convolution-based forced detection, many options to model the PSF have been used. Du [21] warped a model for parallel hole geometry into the focusing geometry, Tsui [22] and Van Roosmalen [23] employed analytic functions for the representation, and Chun [24] took the response from the detector itself and generalized this, which requires measurements for all distances, isotopes, and collimators. All implementations have their own strengths and weaknesses and the best option will depend on the individual requirements. In the case of ${ }^{99 \mathrm{~m}} \mathrm{Tc}$ modeling, collimator interactions will be minimal and hence all options can be expected to achieve similar results. However, when modeling higher-energy photons (e.g. ${ }^{90} \mathrm{Y},{ }^{166} \mathrm{Ho}$ ), collimator interactions will likely become a major factor. In this setting Monte Carlo-based PSFs are expected to most accurately describe the detector physics.

To achieve the largest FOV in the liver, one option would be to increase the gamma camera size. However, as the discussed collimators are symmetric, the gamma camera would at some point collide with the shoulder of the patient. These problems have also been encountered in brain SPECT, where the gamma camera cannot be positioned as close to the skull as preferred, again since the shoulder gets in the way. To solve this problem, a half-cone beam collimator was proposed [25]. Such a tilted collimator could be of use for liver SPECT as well.

The reconstructions performed in this work were made from projections using the same forward projector. This means that the reconstructions will iterate toward the original phantom and are not the same as detector projections [26]. Previously, however, it has been shown that measured detector data match the simulated projections closely [27,28] and hence we assume that these simulated projections provide a good starting point for the investigation of focusing collimators. Future work will focus on the reconstruction of detector measurements. 

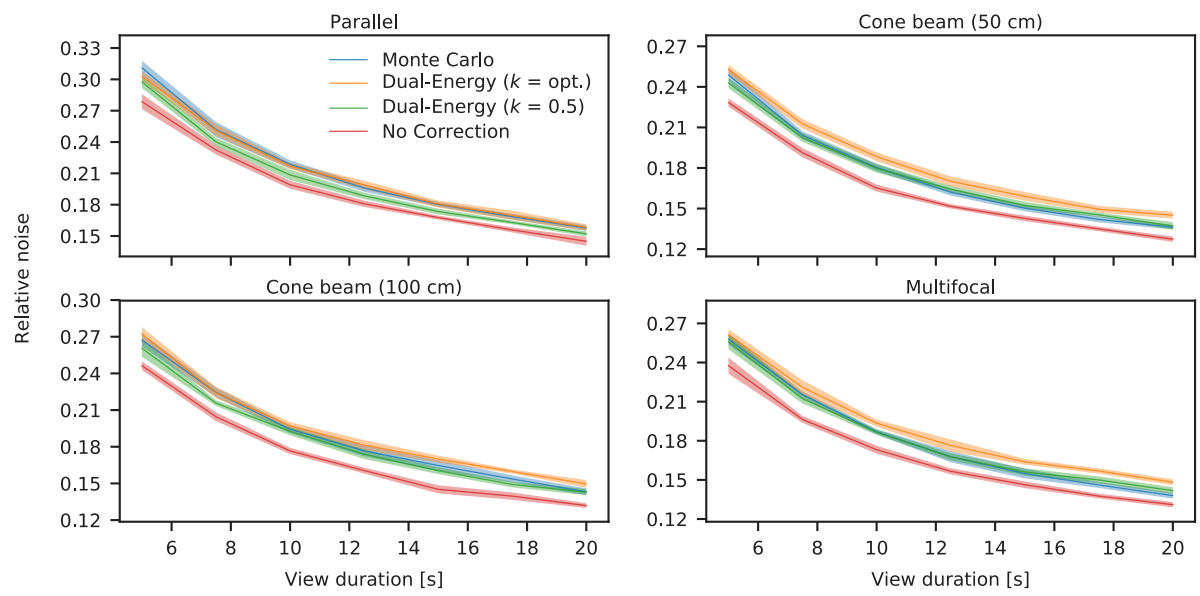

Figure 13: Noise levels, normalized to the mean activity, for the scatter correction methods and for the reconstruction without scatter correction, for the four considered collimators.

Comparison of the collimators using detector measurements will however be challenging, as there exist several parameters that influence the system sensitivity. For example, small production errors in the collimator, differences in photomultiplier tube gains and varying crystal efficiency can all influence the observed noise levels. Furthermore, the used phantom must be configured exactly the same during every measurement. In this study it was therefore decided to only focus on simulations, as this makes it able to only study the effect of collimator choice.

As mentioned in the introduction, a gamma camera in the intervention room would be ideal for image-guided liver SPECT. The patient then does not have to be transferred to the scanning room, which saves time and reduces patient discomfort. Significant effort has recently gone into the development of mobile gamma cameras [29,30]. If such a device were included in the intervention room, the SPECT scan can instantly be made after the scout dose in a short time frame, which would substantially expedite the procedure.

\section{Conclusions}

A fast quantitative Monte Carlo reconstructor for focusing collimators was developed, making reconstruction within minutes feasible. The use of focusing collimators for liver SPECT decreased scanning times. Monte Carlo-based scatter correction traced the tumor activity profiles most accurately. Both fast acquisition and fast quantitative reconstruction are crucial steps in moving toward real-time dosimetry during interventions.

\section{References}

1. Kennedy A, Coldwell D, Sangro B, Wasan H, Salem R. Radioembolization for the Treatment of Liver Tumors. Am J Clin Oncol. 35:91-9 (2012) 
2. Braat AJAT, Smits MLJ, Braat MNGJA, et al. $Y^{90}$ Hepatic Radioembolization: An Update on Current Practice and Recent Developments. J Nucl Med. 56:1079-87 (2015)

3. Gabr A, Kallini JR, Gates VL, et al. Same-day ${ }^{90} \mathrm{Y}$ radioembolization: implementing a new treatment paradigm. Eur J Nucl Med Mol Imaging. 43:2353-9 (2016)

4. Jaszczak RJ, Greer KL, Coleman RE. SPECT using a specially designed cone beam collimator. J Nucl Med. 29:1398-405 (1988)

5. Ter-Antonyan R, Jaszczak RJ, Greer KL, Bowsher JE, Metzler SD, Coleman RE. Combination of converging collimators for high-sensitivity brain SPECT. J Nucl Med. 50:1548-56 (2009)

6. Hawman P, Ghosh P. White Paper IQ SPECT : A Technical and Clinical Overview. (2012)

7. Caobelli F, Kaiser SR, Thackeray JT, et al. IQ SPECT allows a significant reduction in administered dose and acquisition time for myocardial perfusion imaging: evidence from a phantom study. J Nucl Med. 55:2064-70 (2014)

8. De Jong HWAM, Slijpen ETP, Beekman FJ. Acceleration of Monte Carlo SPECT simulation using convolution-based forced detection. IEEE Trans Nucl Sci. 48:58-64 (2001)

9. Elschot M, Lam MGEH, Van den Bosch MAAJ, Viergever MA, De Jong HWAM. Quantitative Monte Carlo-based ${ }^{90}$ Y SPECT reconstruction. J Nucl Med. 54:1557-63 (2013)

10. Elschot M, Smits MLJ, Nijsen JFW, Lam MGEH, et al. Quantitative Monte Carlo-based holmium-166 SPECT reconstruction. Med Phys. 40:112502 (2013)

11. Hawman PC, Haines EJ. The cardiofocal collimator: a variable-focus collimator for cardiac SPECT. Phys Med Biol. 39:439-50 (1994)

12. Siemens Healthcare. Symbia $S$ and T System Specifications. (2010)

13. Segars WP, Sturgeon G, Mendonca S, Grimes J, Tsui BMW. 4D XCAT phantom for multimodality imaging research. Med Phys. 37:4902-15 (2010)

14. Zeng GL, Hsieh YL, Gullberg GT. A rotating and warping projector/backprojector for fan-beam and cone-beam iterative algorithm. IEEE Trans Nucl Sci. 41:2807-11 (1994)

15. Jan S, Santin G, Strul D, et al. GATE: A simulation toolkit for PET and SPECT. Phys Med Biol. 49:4543-61 (2004)

16. Jaszczak RJ, Greer KL, Floyd CE, Harris CC, Coleman RE. Improved SPECT quantification using compensation for scattered photons. J Nucl Med. 25:893-900 (1984)

17. Elschot M, Nijsen JFW, Lam MGEH, et al. ${ }^{99} \mathrm{~m} T \mathrm{Tc}-\mathrm{MAA}$ overestimates the absorbed dose to the lungs in radioembolization: a quantitative evaluation in patients treated with ${ }^{166} \mathrm{Ho}$-microspheres. Eur J Nucl Med Mol Imaging. 41:1965-75 (2014)

18. Braat AJAT, Prince JF, Van Rooij R, Bruijnen RCG, Van den Bosch MAAJ, Lam MGEH. Safety analysis of holmium-166 microsphere scout dose imaging during radioembolisation work-up: A cohort study. Eur Radiol. 28:920-8 (2018)

19. Bastiaannet R, Viergever MA, De Jong HWAM. Impact of respiratory motion and acquisition settings on SPECT liver dosimetry for radioembolization. Med Phys. 44:5270-9 (2017)

20. Li N, Meng L-J. Adaptive Angular Sampling for SPECT Imaging. IEEE Trans Nucl Sci. 58:2205-18 (2011)

21. Du Y, Bhattacharya M, Frey E. Fast Monte Carlo simulation of Siemens IQ-SPECT with SMARTZOOM collimator. J Nucl Med. 53:425 (2012)

22. Tsui BMW, Gullberg GT. The geometric transfer function for cone and fan beam collimators. Phys Med Biol. 35:81-93 (1990) 
23. Van Roosmalen J, Goorden MC. Non-diverging analytical expression for the sensitivity of converging SPECT collimators. Phys Med Biol. 62:228-43 (2017)

24. Chun SY, Fessler JA, Dewaraja YK. Correction for collimator-detector response in SPECT using point spread function template. IEEE Trans Med Imaging. 32:295-305 (2013)

25. Li J, Jaszczak RJ, Van Mullekom A, Scarfone C, Greer KL, Coleman RE. Half-cone beam collimation for triple-camera SPECT systems. J Nucl Med. 37:498-502 (1996)

26. Kaipio J, Somersalo E. Statistical inverse problems: Discretization, model reduction and inverse crimes. J Comput Appl Math. 198:493-504 (2007)

27. Xiao J, De Wit TC, Staelens SG, Beekman FJ. Evaluation of 3D Monte Carlo-based scatter correction for ${ }^{99 m} T c$ cardiac perfusion SPECT. J Nucl Med. 47:1662-9 (2006)

28. Van Gils CAJ, Beijst C, Van Rooij R, De Jong HWAM. Impact of reconstruction parameters on quantitative I-131 SPECT. Phys Med Biol. 61:5166-82 (2016)

29. Beijst C, Elschot M, Viergever MA, De Jong HWAM. Toward Simultaneous Real-Time Fluoroscopic and Nuclear Imaging in the Intervention Room. Radiology. 278:232-8 (2016)

30. Van der Velden S, Kunnen B, Koppert WJC, et al. A dual layer detector for simultaneous fluoroscopic and nuclear imaging. Radiology 290(3):833-838 (2019) 



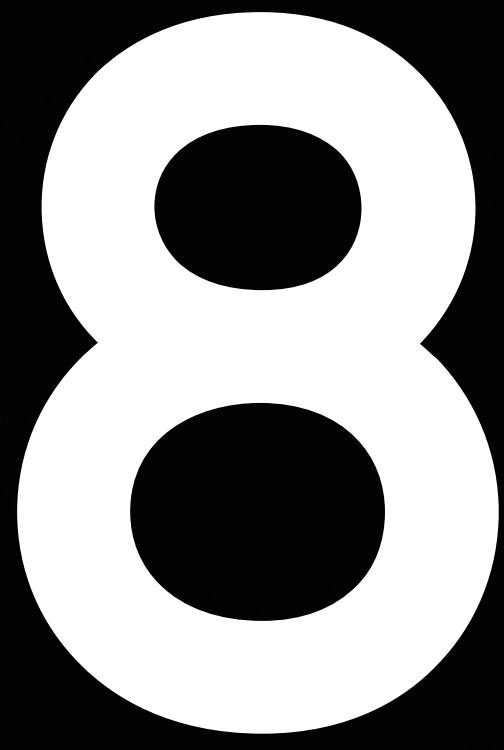




\section{MULTIFOCAL COLLIMATOR IMAGING VALIDATION}

PUBLISHED AS:

MMA Dietze, B Kunnen, M Stella, HWAM de Jong

Monte Carlo-based Scatter Correction for the SMARTZOOM Collimator 


\begin{abstract}
Background

Myocardial perfusion imaging is a commonly performed SPECT protocol and hence it would be beneficial if its scan duration could be shortened. For traditional gamma cameras, two developments have separately shown to allow for a shortened scan duration: i) reconstructing with Monte Carlo-based scatter correction instead of dual-energy window scatter correction and ii) acquiring projections with the SMARTZOOM collimator instead of a parallel-hole collimator. This study investigates which reduction in scan duration can be achieved when both methods are combined in a single system.
\end{abstract}

\title{
Results
}

The SMARTZOOM collimator was implemented in a Monte Carlo-based reconstruction package and the implementation was validated through image quality phantom experiments. The potential for scan duration reduction was evaluated with a phantom configuration that is realistic for myocardial perfusion imaging. The original reconstruction quality was achieved in $76 \pm 8 \%$ of the original scan duration when switching from dual-energy window scatter correction to Monte Carlo-based scatter correction. The original reconstruction quality was achieved in $56 \pm 13 \%$ of the original scan duration when switching from the parallel-hole to the SMARTZOOM collimator. After combining both methods in a single system, the original reconstruction quality was achieved in $34 \pm 7 \%$ of the original scan duration.

\section{Conclusions}

Monte Carlo-based scatter correction combined with the SMARTZOOM collimator can further decrease the scan duration in myocardial perfusion imaging. 


\section{Background}

Myocardial perfusion imaging is a commonly performed technetium- $99 \mathrm{~m}\left({ }^{99 \mathrm{~m}} \mathrm{Tc}\right)$ single-photon emission computed tomography (SPECT) protocol [1]. Scan duration reduction for this protocol has been a major research theme because potential time-gain would allow for substantial increases in the scanner throughput. The duration of a scan can e.g. be shortened through hardware design choices (e.g. by trying to increase the system sensitivity) or improvements in the reconstructed image quality (so that the original quality can be achieved with fewer counts) [2]. We will discuss two successful examples that have been introduced for traditional (i.e. general-purpose) gamma cameras.

First, the SMARTZOOM collimator has been introduced in clinical practice by Siemens Healthineers (Erlangen, Germany) on IQ-SPECT systems [3,4]. This collimator has a converging (i.e. high sensitivity) inner part with diverging (low sensitivity) outer regions. By aiming the converging part of the collimator on the heart (in the 'sweet spot'), up to $4 \mathrm{x}$ more photons can be collected in comparison with a conventional parallel-hole collimator. The diverging parts of the collimator ensure that photons originating from outside of the heart will not produce truncation artifacts in the reconstruction. Since this collimator design provides a higher local sensitivity, it is frequently used to decrease the scan duration [5].

Second, the reconstruction image quality can be improved by implementing sophisticated models for the photon physics (e.g. point spread function modeling and attenuation correction) in the reconstruction algorithms. For myocardial perfusion imaging with a parallel-hole collimator, it has been shown that Monte Carlo-based scatter correction generates higher quality images (i.e. a higher contrast) when compared with the conventional dual-energy window scatter correction approach [6,7] because the non-uniform nature of the attenuation is better incorporated and the reconstructions are not deteriorated by additional Poisson noise in the scatter estimate. The improved contrast can be combined with an increased smoothing (e.g. with a Gaussian filter) so that more noise can be accepted in the initial image, which subsequently also allows for a reduction in scan time.

We believe that it is possible to combine Monte Carlo-based scatter correction together with the SMARTZOOM collimator. This combination might lead to an even larger reduction in scan duration. This study will demonstrate the implementation of Monte Carlo-based scatter correction for the SMARTZOOM collimator and will determine the achievable scan duration reduction using phantom experiments.

\section{Methods}

\section{Acquisition}

All phantom scans were performed on a dual-head Siemens Symbia T2 SPECT/CT scanner with our current clinical scan protocol for myocardial perfusion imaging. Projections were acquired at a $128 \times 128$ grid with a $4.79 \mathrm{~mm}$ isotropic pixel size. The photopeak window captured photons from 
Parallel-hole

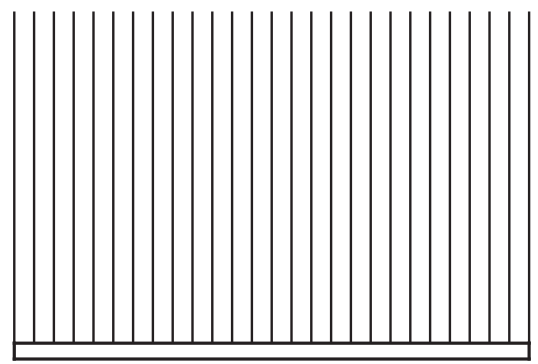

SMARTZOOM

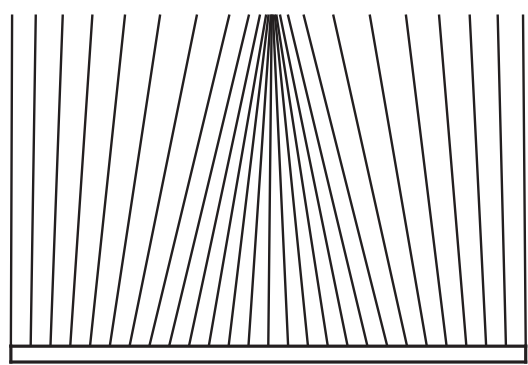

Figure 1: An illustration of the field of view of a conventional parallel-hole collimator and the SMARTZOOM collimator. The highest sensitivity is achieved in the regions with the highest line density.

129 to $150 \mathrm{keV}$ while the scatter window ranged from 108 to $129 \mathrm{keV}$. The attenuation map was created from the low-dose CT scan. The scanner acquired 34 projections (17 per detector head) over 208 degrees (with a start angle of 54 degrees) in step-and-shoot mode. The experiments were performed with two collimators:

\section{Parallel-hole}

The low-energy high-resolution (LEHR) parallel-hole collimator has a uniform sensitivity over its entire surface and is conventionally used for ${ }^{99 \mathrm{~m}} \mathrm{Tc}$ imaging. The collimator had a hole length of $24.05 \mathrm{~mm}$, a hole diameter of $1.11 \mathrm{~mm}$, and a septal thickness of $0.16 \mathrm{~mm}$ [8]. The detector heads performed a body-tracing orbit.

\section{SMARTZOOM}

The SMARTZOOM collimator differs from the parallel-hole collimator by having a converging part in the middle of the collimator, while the outer parts are diverging (see Fig. 1 for an illustration). The converging part increases the sensitivity, while the diverging parts ensure that no truncation artifacts arise in the reconstruction. The hole length of the SMARTZOOM collimator was 40.25 $\mathrm{mm}$, the inner hole diameter was $1.95 \mathrm{~mm}$, and the septal thickness was between 0.2 and $0.4 \mathrm{~mm}$ [8]. The SMARTZOOM collimator is difficult to manufacture because of the complicated geometry and hence every SMARTZOOM collimator will have slightly different angles. These angles have been measured and this information is provided by the manufacturer. For the SMARTZOOM collimators that were present on the two detector heads of the SPECT/CT, the focal lengths were 54.5 and $53.2 \mathrm{~cm}$. The detector heads performed a cardio-centric orbit with a fixed center-todetector distance of $28 \mathrm{~cm}$.

\section{Reconstruction}

The phantom acquisitions were reconstructed with our in-house developed maximum-likelihood expectation-maximization (MLEM) reconstruction software package Utrecht Monte Carlo System (UMCS), which has been validated for a variety of isotopes $[9,10]$. UMCS accounts for photon attenuation via correction with the attenuation map (obtained from the low-dose CT) and for 


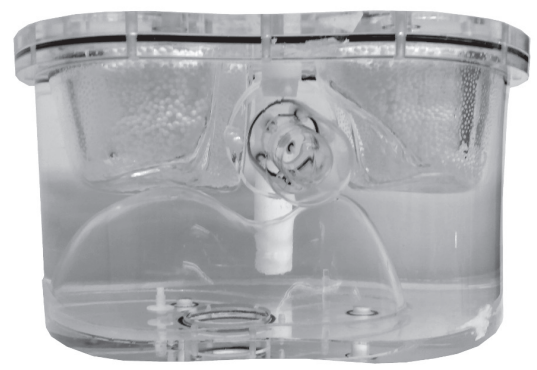

Figure 2: The anthropomorphic phantom that comprises a cardiac insert with a defect.

collimator resolution via distance-dependent point spread function (PSF) modeling [11]. The performance of two scatter correction methods was compared:

\section{Dual-energy window}

Dual-energy window scatter correction, in which the scatter window projections are added in the reconstruction loop at a certain scatter multiplier factor [12], is conventionally used in clinical reconstruction software because of its straightforward implementation. This approach, however, has two drawbacks. First, the scatter multiplier factor is chosen to represent an average scatter medium (often, and also used in this study: $k=0.5$ ), which leads to sub-optimal performance for patients with a non-average physique (e.g. with a very small or large body-mass index). And second, the scatter projections are corrupted by Poisson noise, which increases the noise level in the reconstruction. This latter effect can (partly) be compensated for by filtering the projections, but the optimal filtering magnitude to apply depends heavily on the phantom configuration and was hence not employed in this study.

\section{Monte Carlo-based}

A more sophisticated method for scatter correction is to simulate the physical interactions of the photons in the patient. This is performed by Monte Carlo-based sampling of photon emissions from the intermediate reconstructed activity image and determining their traveling distance, traveling angle, and energy loss when an interaction (at a specific scatter position) with the scattering medium (the attenuation map) occurs. This process is hence repeated for $2^{\text {nd }}, 3^{\text {rd }}$, etc. orders of scatter until the photon is either absorbed or falls below an energy limit. The photon physics simulation can take a long time but is greatly accelerated with variance reduction techniques. Convolution-based forced detection is employed for this purpose in UMCS. In convolution-based forced detection, at every scatter position the probability is calculated that the photon scatters towards the detector and is detected. In addition, instead of adding one detection event to the projection, all the potential events are projected using a predefined and tabulated kernel. It was previously determined that the change in the simulated projections became very small after the simulation of $5 \times 10^{6}$ photons [13] and hence this number of simulated photons was used per projection. The simulated scatter projections are added in the reconstruction loop similarly as is done in the dual-energy window approach. 
Table 1: The organ activity concentrations in the myocardial perfusion phantom.

\begin{tabular}{l|ccc} 
& Volume $[\mathrm{mL}]$ & Concentration $[\mathrm{kBq} / \mathrm{mL}]$ & Activity $[\mathrm{MBq}]$ \\
\hline Liver & 1160 & 94 & 109.0 \\
Background & 8838 & 7.6 & 67.2 \\
Heart & 118 & 159 & 18.8
\end{tabular}

The variable field of view of the SMARTZOOM collimator was implemented in UMCS by warping the intermediate reconstruction volumes and the attenuation map during the forward and backward projection step of the reconstruction [14] (using the collimator hole angles that are provided by the scanner manufacturer) so that the rays under an angle become parallel to the detector. With this approach, it becomes possible to perform the further forward and backward projection operations (e.g. weighted summing of the image volume to obtain the projection) the same as is done with parallel-hole collimators. This approach is relatively easy to implement and has the additional benefit that only a single point spread function (as a function of the source-to-detector distance) is required since the detector response is approximately shift-invariant for translations in the detector plane (after warping) [15]. Since the septal thickness of the SMARTZOOM collimator ranged between 0.2 and $0.4 \mathrm{~mm}$, point spread functions were created for an average septal thickness of 0.3 $\mathrm{mm}$.

\section{Validation}

To verify whether our reconstruction implementation of the SMARTZOOM collimator was accurate, we compared the reconstructions obtained from UMCS (with dual-energy window correction) with reconstructions from the clinical software. Two image quality phantoms were used for this task:

- A rack with five point sources (positioned in the same plane) of approximately $29 \mathrm{MBq}$ ${ }^{99} \mathrm{~m}$ Tc each that was scanned with 30 seconds per projection. This phantom was used to check whether the point sources were reconstructed to the same position (validating the warping methodology) and to determine whether they were reconstructed as small point sources (validating the point-spread function implementation).

- A cylinder (6820 mL volume) filled uniformly with $148 \mathrm{MBq}{ }^{99 m} \mathrm{Tc}$ that was scanned with 30 seconds per projection. This phantom was used to evaluate the uniformity of the reconstruction (validating the attenuation correction approach) and the reconstruction noise patterns (which are dependent on the used reconstruction algorithm).

The clinical reconstructions employed the MLEM reconstruction algorithm for the parallelhole collimator but used the ordered subset conjugate gradient minimizer (OSCGM) for the SMARTZOOM collimator. The reconstructions were, for both collimators, made with 30 iterations. No subsets were used because of the relatively low number of projection angles available. 


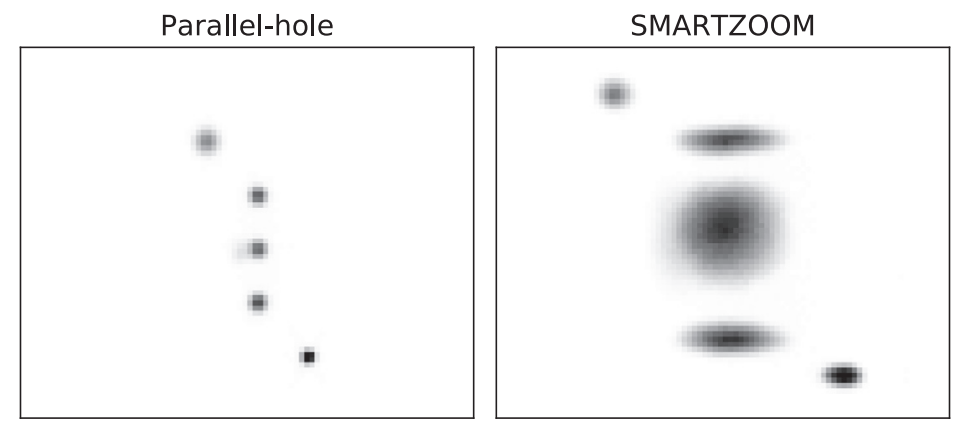

Figure 3: Projections of the five ${ }^{99 \mathrm{~m}} \mathrm{Tc}$ point sources obtained with the parallel-hole and the SMARTZOOM collimator.

The UMCS reconstructions used the MLEM reconstruction algorithm for both collimators. The respective iteration numbers were chosen by visually determining the best-matching images with the clinical reconstructions. This resulted in 40 iterations for the parallel-hole collimator and 250 iterations for the SMARTZOOM collimator. The UMCS reconstructions for the SMARTZOOM collimator required substantially more iterations than the clinical software due to faster convergence of the OSCGM algorithm compared to MLEM [16].

Both the clinical and the UMCS reconstructions were made with attenuation correction, point spread function modeling, and dual-energy window scatter correction. The reconstructions were made on a $128 \times 128 \times 128$ grid with a $4.79 \mathrm{~mm}$ isotropic pixel size and a $5 \mathrm{~mm}$ full-width at halfmaximum (FWHM) Gaussian post-reconstruction filter was applied.

\section{Scan duration reduction}

The potential for scan duration reduction was evaluated for the primary task in myocardial perfusion imaging: the detection of a cardiac defect. The most accurate detection can be made when: i) the contrast between the healthy tissue and the defect is as large as possible, and ii) the noise in the background is as low as possible. The reconstructions of the collimator and scatter correction combinations (made with 100 iterations and no post-reconstruction filter) will hence be compared on a metric that combines both of these measures: the contrast-to-noise ratio (CNR).

\section{Contrast}

The contrast was determined in an anthropomorphic phantom (model ECT/TOR/P) that comprised a cardiac insert (model ECT/CAR/I) with a defect of $2 \mathrm{~mL}$ (i.e. with no activity present) (see Fig. 2). The phantom was filled with a ${ }^{99 \mathrm{~m}} \mathrm{Tc}$ activity distribution that is clinically encountered in myocardial perfusion imaging (see Tab. 1) [5,17]. The phantom was scanned with a relatively large acquisition time of 90 seconds per projection to obtain low-noise projections. The hot wall and the defect were manually delineated on the SPECT reconstructions and the contrast was calculated as:

$$
C=\left(I_{w}-I_{d}\right) / I_{d}
$$

where $I_{w}$ is the intensity in the hot wall and $I_{d}$ is the intensity in the defect. 

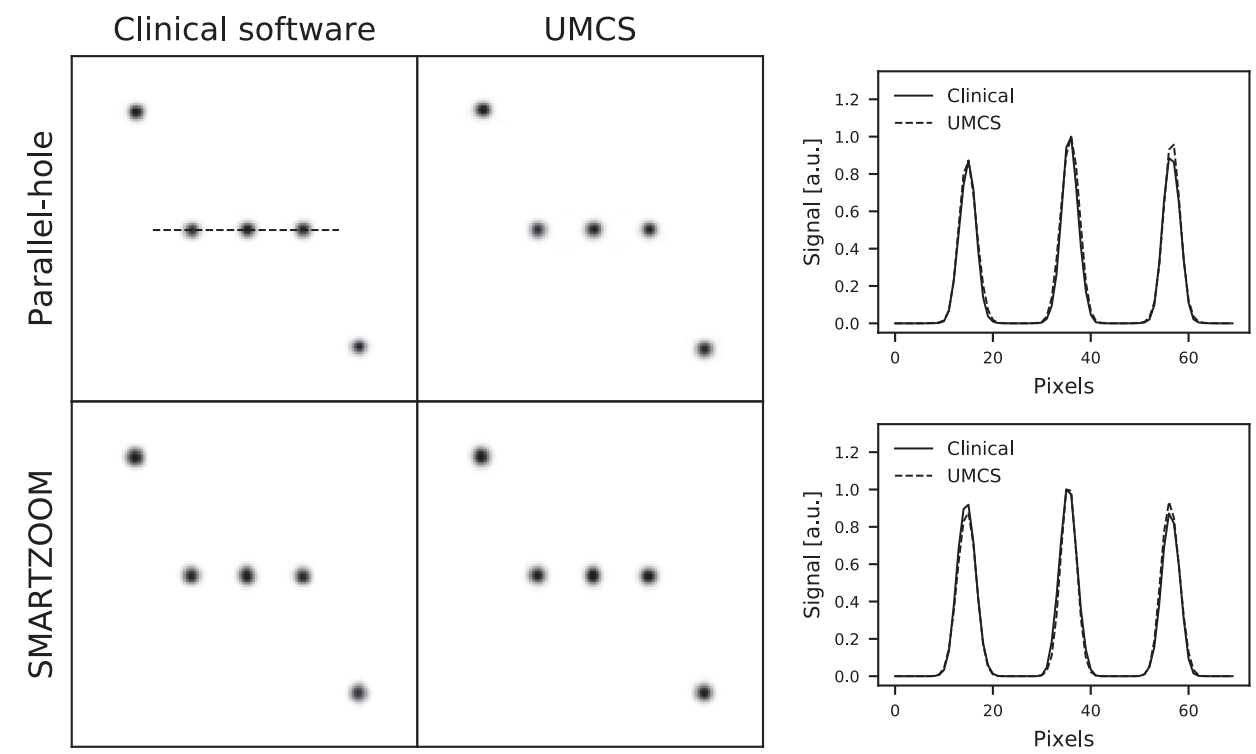

Figure 4: The reconstructions of the point sources obtained with the parallel-hole and the SMARTZOOM collimator when reconstructed with the clinical software and UMCS shown together with the profile at the location of the dashed line in the upper left reconstruction.

Noise

The noise (calculated as the standard deviation in the volume of interest) should ideally be determined in a relatively large volume that is not corrupted by resolution effects. Normally, one could e.g. determine the noise in the background volume of the anthropomorphic phantom. For the SMARTZOOM collimator, however, we are only interested in the noise that is present in the 'sweet spot' and hence this phantom could not be used.

Instead, the noise was determined in the 'sweet spot' of the cylinder phantom (that was used for the reconstruction validation) with its total number of counts scaled to the background activity concentration of the myocardial perfusion phantom at a clinical activity level $(100 \mathrm{MBq}$, from $800 \mathrm{MBq}$ injected activity in combination with 8 gating frames) when scanned with a clinical scan duration ( 20 seconds per projection). These sampled projections were created by putting all events of the original projections in a list, performing a random shuffle of the list, and taking the first $12 \%$ of the events. Since the SPECT reconstructions are sensitive to noise patterns for these low-count projections, the sampling and reconstruction process was repeated 20 times to obtain statistically significant results.

\section{Contrast-to-noise}

Normally, the CNR is determined at a set number of iterations. In this work, however, this is not feasible since the convergence speed between the several collimator and scatter correction combinations varies substantially. Instead, the maximum achieved CNR over the iterations was collected so that the convergence speed was no longer of influence. 

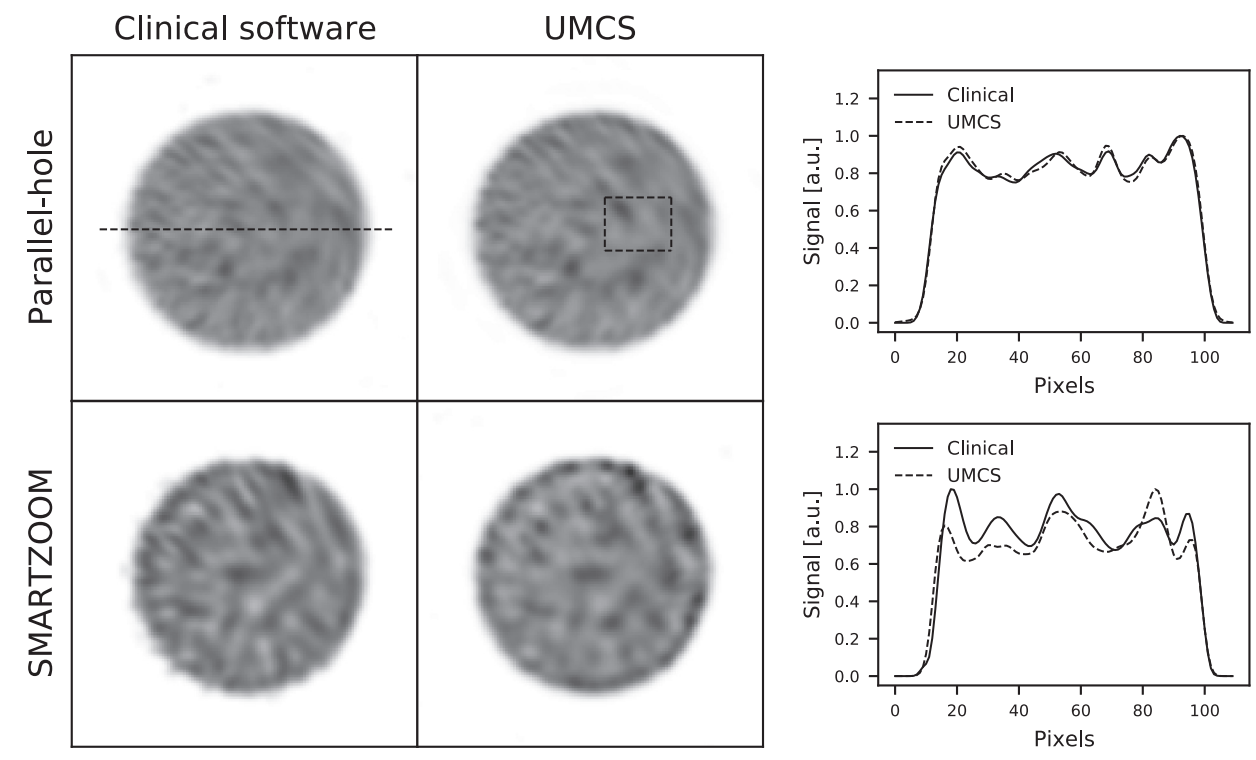

Figure 5: The reconstructions of the uniformly filled cylinder obtained with the parallel-hole and the SMARTZOOM collimator when reconstructed with the clinical software and UMCS shown together with the profile at the location of the dashed line in the upper left reconstruction. The dashed rectangle in the upper right reconstruction represents the volume in which the noise was calculated for the CNR analysis.

To determine the scan duration reduction that can be achieved, the scaled cylinder projections were further sub-sampled to obtain projections that would be obtained with 95 to $5 \%$ (in steps of $5 \%$ ) of the original scan duration. The maximum CNR values were collected for every scan duration and it was determined at which scan durations an equal reconstruction quality was achieved.

\section{Results}

\section{Validation}

Projections of the ${ }^{99 \mathrm{~m}} \mathrm{Tc}$ point sources obtained with the parallel-hole and the SMARTZOOM collimator are shown in Fig. 3. The point spread function of the SMARTZOOM collimator has a spatial variance over the collimator surface: the PSF is largest in the middle of the collimator (where both directions converge) and smallest in the collimator corners (where both directions diverge). The remaining areas have an asymmetric PSF since these parts have convergence in one direction and divergence in the other.

The reconstructions of the point sources are shown in Fig. 4 together with their profiles. The point sources reconstructed to the same location and were approximately the same size. This result demonstrates that the warping and the point spread function modeling were correctly implemented for the SMARTZOOM collimator geometry.

The reconstructions of the uniformly filled cylinder are shown in Fig. 5 together with their profiles. The noise patterns are similar in the clinical software and UMCS in the case of the parallel-hole 


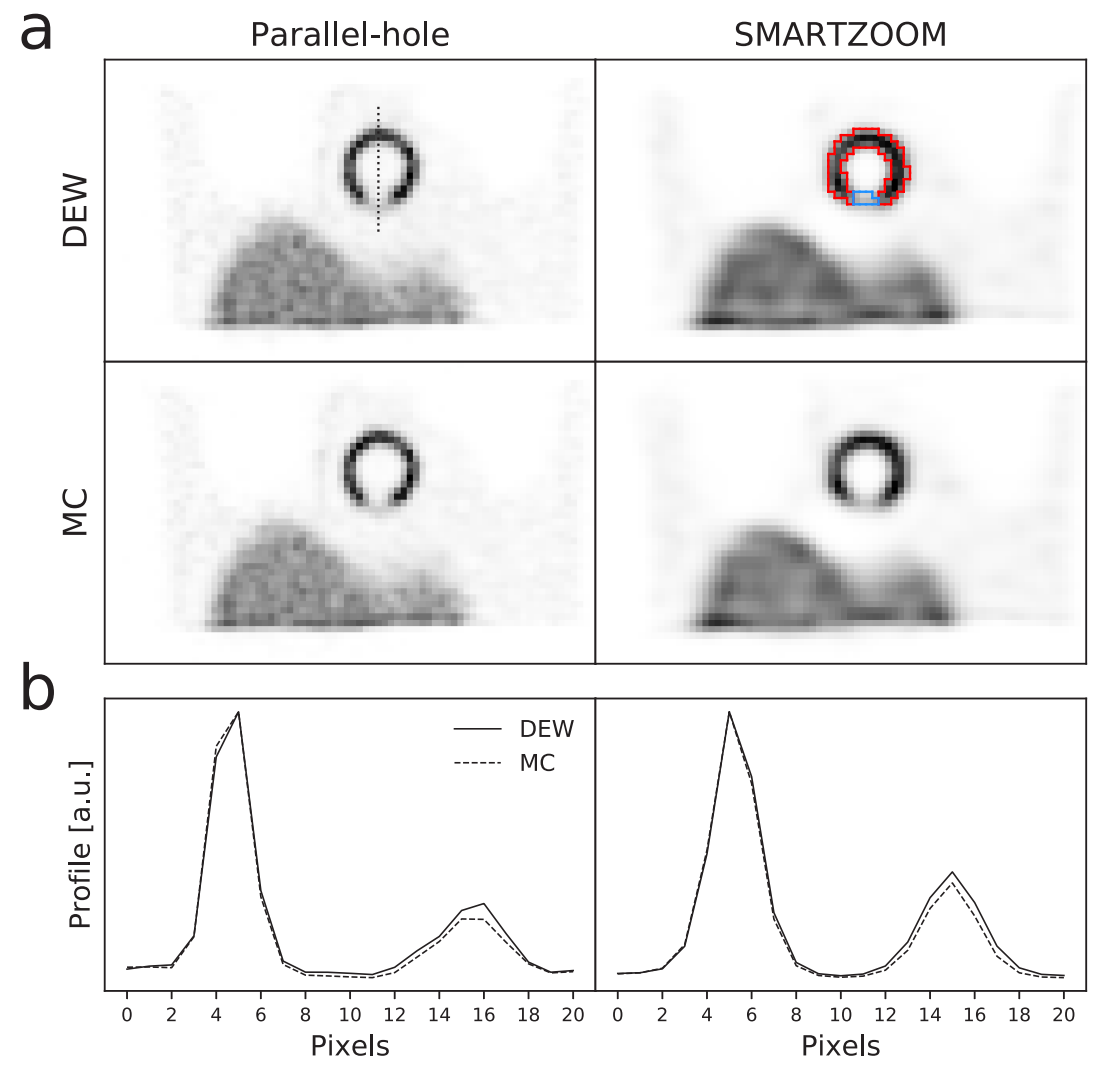

Figure 6: (a) The reconstructions of the myocardial perfusion phantom at iteration 100 obtained with the parallel-hole and the SMARTZOOM collimator when reconstructed with dual-energy window and Monte Carlo-based scatter correction. The dotted line in the upper left reconstruction represents the location of the profiles. The red lines in the upper right reconstruction represent the mask of the hot wall and the blue lines represent the mask of the defect. (b) The profiles over the cardiac insert. The left and the right (lower) peaks are the hot wall and the defect, respectively.

collimator. For the SMARTZOOM collimator, the patterns were different because of the different reconstruction algorithms used (OSCGM versus MLEM). All reconstructions showed a uniform distribution, which demonstrates that the attenuation correction was correctly implemented. We conclude that our implementation of the SMARTZOOM collimator is accurate.

\section{Scan duration reduction}

\section{Contrast}

The reconstructions of the myocardial perfusion phantom at iteration 100 are shown in Fig. 6 for all collimator and scatter correction combinations, together with the profiles over the cardiac insert with the defect. The profiles illustrate that Monte Carlo-based scatter correction achieves a slightly better contrast (difference in intensity between the hot wall and the defect) than dual-energy window scatter correction for both the parallel-hole and the SMARTZOOM collimator. 

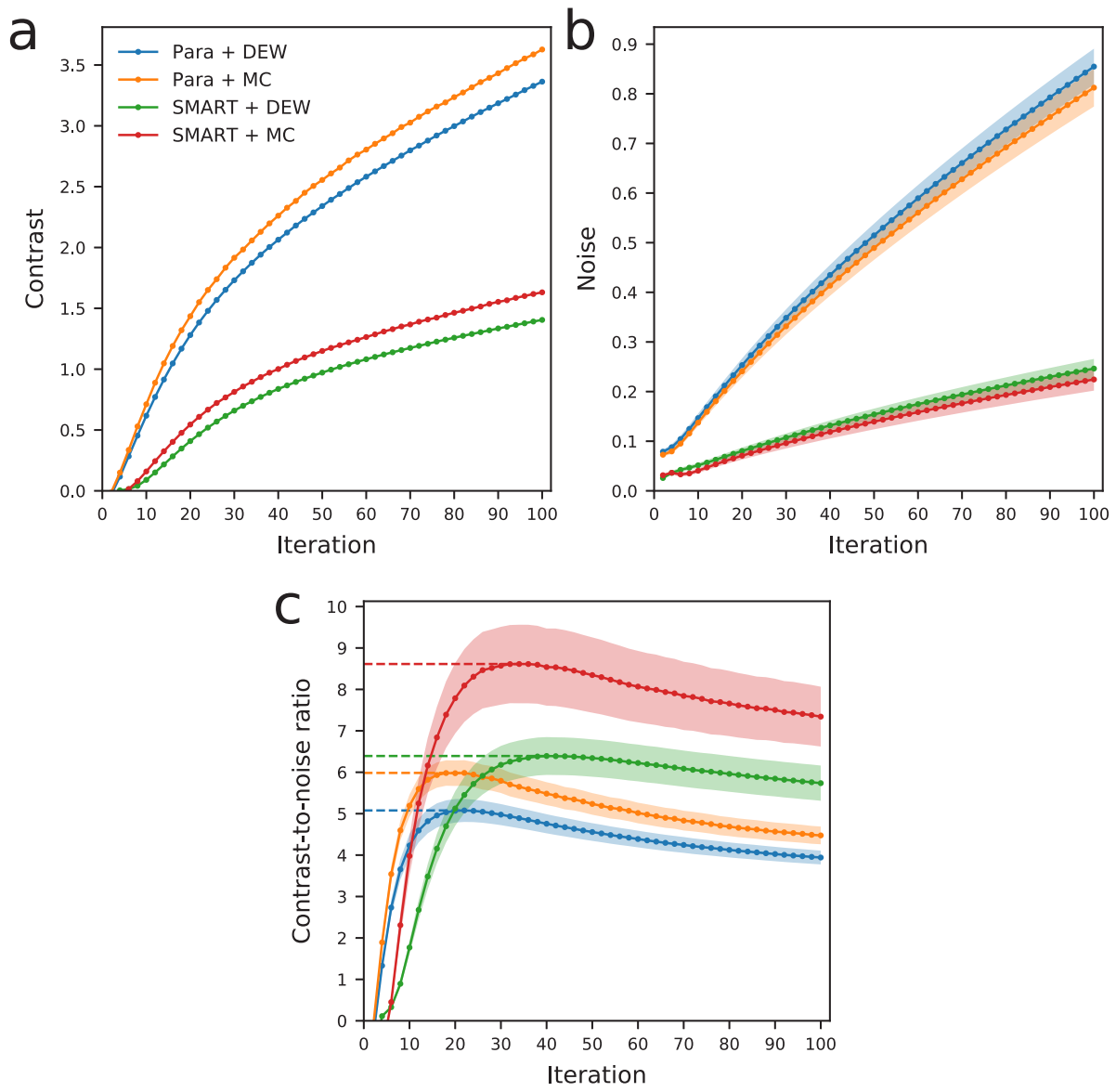

Figure 7: The (a) contrast, (b) noise, and (c) contrast-to-noise ratios, as a function of the iteration number. The shaded regions in (b) and (c) represent the standard deviation over the 20 noise realizations. The dotted lines in (c) indicate the maximum achieved CNR values.

The contrast, as a function of the iteration number, is shown in Fig. 7a. This figure illustrates that the SMARTZOOM collimator has a lower contrast than the parallel-hole collimator at the same iteration. This occurs because of the slower convergence of the SMARTZOOM collimator (due to the intermediate warping during the reconstruction) and because of the worse resolution (due to the relatively larger detector-source distance).

\section{Noise}

The noise, as a function of iteration number, is shown in Fig. 7b. The SMARTZOOM collimator has a lower noise level than the parallel-hole collimator because it captures up to $4 \mathrm{x}$ more photons in the 'sweet spot' and because it convergences slower. The reconstructions made with Monte Carlobased scatter correction result in a slightly lower noise level than those made with dual-energy window scatter correction. This is explained by the almost noiseless scatter projections in the case of Monte Carlo-based scatter correction whereas the scatter projections are corrupted by Poisson noise in the case of dual-energy window correction. 


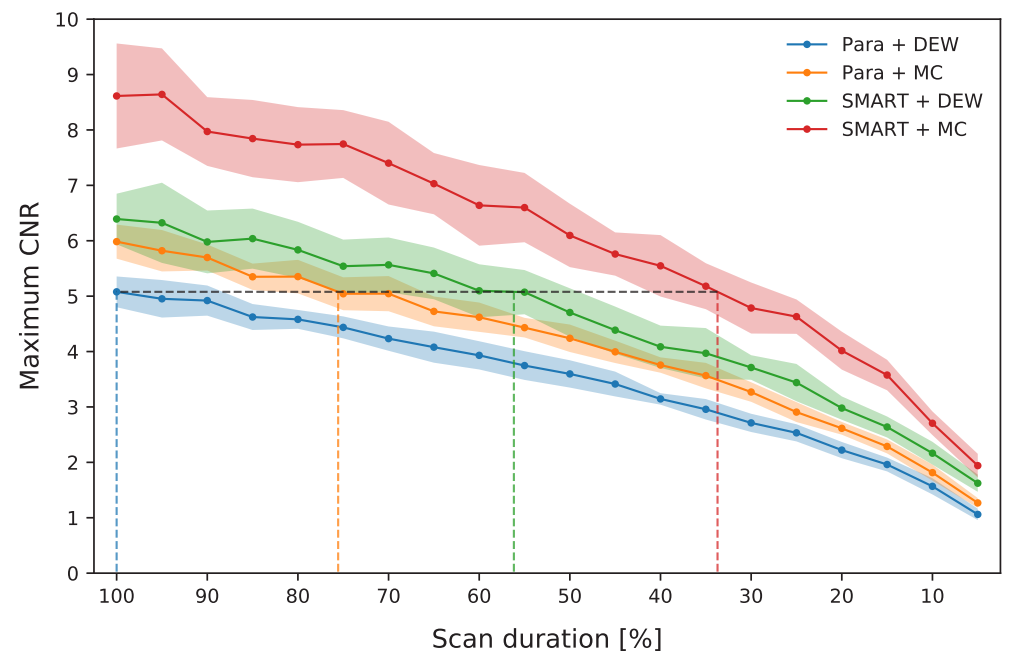

Figure 8: The maximum CNR, as a function of the scan duration. The black dashed line is drawn at the maximum CNR achieved by the parallel-hole collimator with dual-energy window scatter correction. The dashed lines in color are drawn at the scan durations at which the same maximum CNR is achieved for the other collimator and scatter correction combinations. The shaded areas represent the standard deviation over the 20 noise realizations.

\section{Contrast-to-noise}

The contrast-to-noise ratio, as a function of iteration number, is shown in Fig. 7c. The CNR reaches a maximum value for all collimator and scatter correction combinations: after this point, the increase in reconstruction noise starts to dominate over the further gain in contrast. The maximum value is achieved at a different iteration number for every combination.

The best image quality, i.e. the highest CNR, is achieved for the SMARTZOOM collimator with Monte Carlo-based scatter correction. The lowest image quality is achieved for the parallel-hole collimator with dual-energy window scatter correction.

The maximum achieved CNR, as a function of the scan duration, is shown in Fig. 8. The maximum CNR decreases when the scan duration is shortened since the projection noise becomes greater. It was determined at which scan duration the same reconstruction quality as that of the parallel-hole collimator with dual-energy window scatter correction was achieved. This resulted in the following results:

- By moving from dual-energy window to Monte Carlo-based scatter correction, the same reconstruction quality was achieved in $76 \pm 8 \%$ of the original scan duration

- By moving from the parallel-hole to the SMARTZOOM collimator, the same reconstruction quality was achieved in $56 \pm 13 \%$ of the original scan duration

- By combining Monte Carlo-based scatter reconstruction and the SMARTZOOM collimator in a single system, the same reconstruction quality was achieved in $34 \pm 7 \%$ of the original scan duration 


\section{Discussion}

This study investigated the potential for Monte Carlo-based scatter correction in combination with the SMARTZOOM collimator for myocardial perfusion imaging and demonstrated that the scan duration could be shortened even further.

There are three limitations to the current study. First, only one cardiac phantom configuration was studied. The relative performance of the collimator and scatter correction combinations will be different when other activity distributions are imaged. The parallel-hole collimator will perform relatively better when smaller defects need to be visualized (due to its better resolution). The SMARTZOOM collimator will perform relatively better when the activity injected in the patient is lower (due to its better sensitivity). Second, only the contrast-to-noise ratio in the reconstructions was evaluated as a measure of detectability. In certain situations, it may be beneficial to have a better spatial resolution in the reconstruction rather than a lower noise level. And third, no clinical evaluation was performed. The performance in a clinical setting may be different than with phantom experiments because of patient movements (including respiratory and cardiac motion) and non-uniform activity distributions. For a full comparison between the parallel-hole and the SMARTZOOM collimator, a study needs to be performed that investigates more (clinical) activity distributions and calculates more measures of detectability.

There are various practical considerations when employing dual-energy window correction. The scatter multiplier factor can be set higher or lower, the scatter window can be set broader or narrower, and the scatter projection can be filtered or not. In this manuscript, the current clinical settings in our institute were used (scatter multiplier of $k=0.5$, a scatter window from 108 to 129 $\mathrm{keV}$, and no filtering of the scatter projections). For the scatter multiplier factor, it was calculated with the Monte Carlo-based scatter simulator that the optimal $k$-values (based on the total number of counts) were 0.58 for the parallel-hole and 0.56 for the SMARTZOOM collimator. For the scatter window range and the filtering of the projections, the changes in these values will generally be a trade-off between reconstruction noise and contrast. The optimal settings for the dual-energy window settings will depend on the activity distribution configuration and cannot be optimized for every patient separately in clinical practice. An advantage of Monte Carlo-based scatter correction is that the above values do not need to be set.

The time required for reconstruction differs for the studied scatter correction and collimator combinations. A disadvantage of Monte Carlo-based scatter correction over window-based correction is that more computational power is required for the simulation of the photon physics. A disadvantage of the SMARTZOOM collimator over the parallel-hole collimator is that more computational power is required for the forward and backward warping steps during the reconstruction. In the current non-optimized implementation $(3.1 \mathrm{GHz}$ processor), the time required for 100 iterations was $70 \mathrm{~min}$ for the parallel-hole collimator with dual-energy window scatter correction, $108 \mathrm{~min}$ for the parallel-hole collimator with Monte Carlo-based scatter correction, $126 \mathrm{~min}$ for the SMARTZOOM collimator with dual-energy window scatter correction, and 153 min for the SMARTZOOM collimator with Monte Carlo-based scatter correction. 
The difference in the myocardial perfusion phantom profiles between dual-energy window scatter correction and Monte Carlo-based scatter correction was relatively small. To evaluate whether the difference was significant, 20 projections were randomly sampled from the long projection (each projection containing 50\% of the counts) and individually reconstructed. The contrast was calculated for every reconstruction and the average contrast with the associated standard deviation was determined. This investigation demonstrated that, in line with previous studies [6,7], the contrast of Monte Carlo-based scatter correction was significantly higher (two-sided t-test, $p<$ 0.05) than that of dual-energy window correction.

The reconstructions made with Monte Carlo-based scatter correction had an improved contrast over the reconstructions made with dual-energy window scatter correction. We used this property to shorten the scan duration. However, there could also be value in having an increased reconstruction image quality, e.g. by being able to better detect smaller myocardial defects. An observer study would need to be performed to evaluate whether such sharper reconstructions indeed provide more diagnostic information.

Monte Carlo-based scatter correction was studied for the SMARTZOOM collimator because this was the only focusing collimator dedicated to cardiac imaging in our institute. However, we expect that other focusing collimators (such as the cone beam and the fan beam collimators) that are employed in cardiac imaging could similarly benefit from Monte Carlo-based scatter correction.

The implementation of realistic photon interaction physics in the forward projector does not only allow for improved scatter correction but also opens up the possibility of studies with more complicated photon spectra, such as in multi-isotope studies [18]. For instance, Du et al. [19] studied the performance of simultaneous ${ }^{99 \mathrm{~m}} \mathrm{Tc} /{ }^{123} \mathrm{I}$ imaging in combination with the SMARTZOOM collimator. Such studies can be used to potentially gain more information on cardiac functioning.

\section{Conclusions}

Monte Carlo-based scatter correction and the SMARTZOOM collimator have individually shown to accomplish a scan duration reduction in myocardial perfusion imaging. We demonstrated that their combination can provide an even further scan duration reduction.

\section{References}

1. Dorbala S, Ananthasubramaniam K, Armstrong IS, Chareonthaitawee P, DePuey EG, Einstein AJ, Gropler RJ, Holly TA, Mahmarian JJ, Park MA, Polk DM. Single photon emission computed tomography (SPECT) myocardial perfusion imaging guidelines: instrumentation, acquisition, processing, and interpretation. J Nucl Card. 2018;25(5):1784-846

2. Slomka PJ, Patton JA, Berman DS, Germano G. Advances in technical aspects of myocardial perfusion SPECT imaging. J Nucl Card. 2009;16(2):255-76

3. Hawman PC, Haines EJ. The cardiofocal collimator: a variable-focus collimator for cardiac SPECT. Phys Med Biol. 1994;39(3):439-50

4. Hawman P, Ghosh P. White Paper IQ SPECT : A Technical and Clinical Overview. 2012. 
5. Caobelli F, Kaiser SR, Thackeray JT, Bengel FM, Chieregato M, Soffientini A, et al. IQ SPECT allows a significant reduction in administered dose and acquisition time for myocardial perfusion imaging: evidence from a phantom study. J Nucl Med. 2014;55(12):2064-70

6. Xiao J, De Wit TC, Staelens SG, Beekman FJ. Evaluation of 3D Monte Carlo-based scatter correction for ${ }^{99 m}$ Tc cardiac perfusion SPECT. J Nucl Med. 2006;47(10):1662-1669

7. Xiao J, De Wit TC, Zbijewski W, Staelens SG, Beekman FJ. Evaluation of 3D Monte Carlo-based scatter correction for ${ }^{201} \mathrm{Tl}$ cardiac perfusion SPECT. J Nucl Med. 2007;48(4):637-644

8. Siemens Healthcare. Symbia $S$ and T System Specifications.

9. Elschot M, Lam MGEH, van den Bosch MAAJ, Viergever MA, de Jong HWAM. Quantitative Monte Carlo-based ${ }^{90}$ Y SPECT reconstruction. J Nucl Med. 2013;54(9):1557-63

10. Elschot M, Smits MLJ, Nijsen JFW, Lam MGEH, Zonnenberg BA, van den Bosch MAAJ, et al. Quantitative Monte Carlo-based holmium-166 SPECT reconstruction. Med Phys. 2013;40(11):112502

11. De Jong HWAM, Slijpen ETP, Beekman FJ. Acceleration of Monte Carlo SPECT simulation using convolution-based forced detection. IEEE Trans Nucl Sci. 2001;48(1):58-64

12. Jaszczak RJ, Greer KL, Floyd CE, Harris CC, Coleman RE. Improved SPECT quantification using compensation for scattered photons. J Nucl Med. 1984;25(8):893-900

13. de Wit TC, Xiao J, Beekman FJ. Monte Carlo-based statistical SPECT reconstruction: influence of number of photon tracks. IEEE Trans Nucl Sci. 2005;52(5):1365-9

14. Zeng GL, Hsieh YL, Gullberg GT. A rotating and warping projector/backprojector for fan-beam and cone-beam iterative algorithm. IEEE Trans Nucl Sci. 1994;41(6):2807-11

15. Dietze MMA, Van der Velden S, Lam MGEH, Viergever MA, De Jong HWAM. Fast quantitative reconstruction with focusing collimators for liver SPECT. EJNMMI Phys. 2018;5(1):28

16. Onoguchi, M, Konishi, T, Shibutani, T, Matsuo, S, Nakajima, K. Technical Aspects: Image Reconstruction. Ann of Nucl Card. 2016;2(1):68-72

17. Zoccarato O, Scabbio C, De Ponti E, Matheoud R, Leva L, Morzenti S, Menzaghi M, Campini R, Marcassa C, Del Sole A, Garancini S. Comparative analysis of iterative reconstruction algorithms with resolution recovery for cardiac SPECT studies. A multi-center phantom study. J Nucl Card. 2014;21(1):135-48

18. De Jong HWAM, Beekman F, Viergever MA, Van Rijk P. Simultaneous ${ }^{99 \mathrm{~m}} \mathrm{Tc} /{ }^{201} \mathrm{Tl}$ dual-isotope SPET with Monte Carlo-based down-scatter correction. EJNMMI. 2002;29(8):1063-1071

19. Du Y, Bhattacharya M, Frey EC. Simultaneous Tc-99m/I-123 dual-radionuclide myocardial perfusion/ innervation imaging using Siemens IQ-SPECT with SMARTZOOM collimator. Phys Med Biol. 2014;59(11):2813 


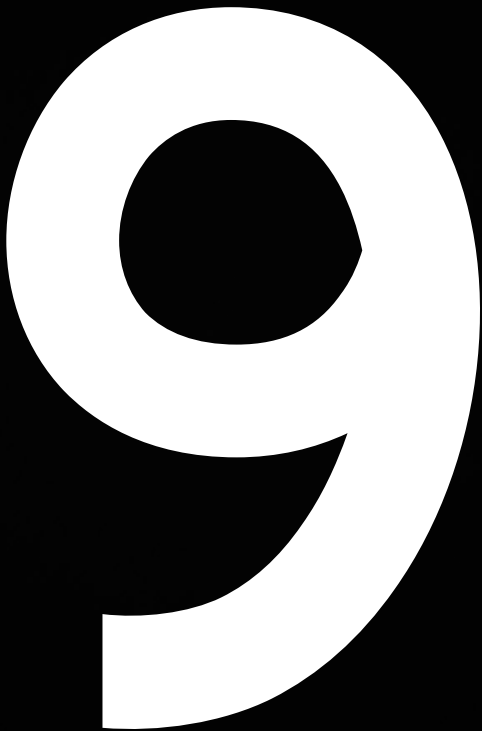




\section{AN ADAPTIVE NUCLEAR SCAN DURATION}

PUBLISHED AS:

MMA Dietze, B Kunnen, C Beijst, HWAM de Jong Adaptive Scan Duration in SPECT: Evaluation for Radioembolization 


\begin{abstract}
Purpose

It may be challenging to select the optimal scan duration for SPECT protocols because the activity distribution characteristics can differ in every scan. Using simulations and experiments, we investigated whether the scan duration can be optimized for every scan separately by evaluating the activity distribution during scanning. We refer to this as adaptive scanning.
\end{abstract}

\title{
Methods
}

The feasibility of adaptive scanning was evaluated for the detection of extrahepatic depositions in the pretreatment procedure of radioembolization, in which ${ }^{99 \mathrm{~m}} \mathrm{Tc}$-labeled macroaggregated albumin ( $\left.{ }^{99 \mathrm{~m}} \mathrm{Tc}-\mathrm{MAA}\right)$ is injected into the liver. We simulated fast one-minute detector rotations and updated the reconstruction with the newly collected counts after every rotation. The scan was terminated when one of two criteria was met: i) when the mask difference of the detected extrahepatic deposition between two consecutive rotations was lower than $5 \%$; or ii) when the reconstructed extrahepatic activity was negligible with respect to the total reconstructed activity $(<0.075 \%)$. The performance of adaptive scanning was evaluated using a digital phantom with various activity distributions, with a physical phantom experiment, and with simulations based on 129 patient activity distributions.

\section{Results}

The digital phantom data showed that the scan termination times substantially depended on the activity distribution characteristics. The experimental phantom data showed the feasibility of adaptive scanning with physical scanner measurements and illustrated that fast detector motion was not limiting the adaptive scanning performance. The patient data showed a large spread in the scan terminations times. By using adaptive scanning, the mean scan duration of the patient distributions was shortened from 20 minutes (current clinical protocol) to $4.8 \pm 0.2$ minutes. The detection accuracy of extrahepatic depositions was unaffected and the mean difference in the extrahepatic deposition masks (compared with the 20 -minute scan) was only $7.0 \pm 1.0 \%$.

\section{Conclusion}

Our study suggests that the SPECT scan duration can be personalized by assessing the activity distribution characteristics during scanning for the detection of extrahepatic depositions in the pretreatment procedure of radioembolization. The adaptive scanning approach might also be of benefit for other SPECT protocols, as long as a measure of interest is available for optimization. 


\section{Introduction}

Selecting the optimal scan duration for a SPECT imaging task remains a challenge because the activity distribution characteristics can differ in every scan. To ensure that the reconstruction answers the imaging question in all cases (i.e. also with low activity or heterogeneous small volumes), scanning is performed with a fixed acquisition time that may be longer than required for most distributions. The scanning efficiency and workflow can be improved if the scan duration is optimized for every activity distribution separately, i.e. by scanning shorter for simple activity distributions and longer for complicated ones. We refer to this as adaptive scanning.

We aim to evaluate an adaptive scanning technique by performing multiple fast detector rotations (of e.g. one minute each) so that the reconstruction can be updated with the extra obtained counts after every rotation. Central to this approach is the existence of a metric of image quality for the task at hand that can be automatically measured. The change of this metric is tracked over the rotations and when its relative change falls below a certain threshold value, the scan is considered to be converged (with respect to the imaging task) and can be terminated. This approach ensures that all activity distributions satisfy the imaging requirement but also that the scan duration is not longer than necessary.

This study evaluates the possibility for such an adaptive scan duration for the pretreatment procedure of hepatic radioembolization, in which ${ }^{99} \mathrm{~m} T \mathrm{Tc}$-labeled macroaggregated albumin ${ }^{99 \mathrm{~m}} \mathrm{Tc}$ $\mathrm{MAA}$ ) is injected into the liver to estimate the activity distribution of the ${ }^{90} \mathrm{Y}$ microspheres [1]. The pretreatment procedure has two objectives in current clinical practice: i) to determine the fraction of activity shunting to the lungs, and ii) to detect potential extrahepatic depositions.

Previously, it was demonstrated that the lung shunt fraction (LSF) can be accurately determined within minutes of scanning (since it is based on the total counts in relatively large volumes) [2] and no further optimization is hence required for this task. However, for the detection of extrahepatic depositions, count statistics become more important. These count statistics are highly dependent on the specific activity distribution characteristics (e.g. the activity in an extrahepatic deposition and the background noise), which can vary considerably between patients. Hence, it is expected that a substantial spread in the required scan durations will be observed for this imaging task.

This study will investigate the influence of the activity distribution characteristics on the scan termination times with a simulation study and the performance in clinically encountered distributions with a retrospective patient study. The fast scanning protocol requires continuous detector motion over the multiple rotations in order to reduce the overhead time when compared with regular step-and-shoot motion. Since the detector orbit can fluctuate over the rotations and the detector distance and detector rotation angle can fluctuate within one projection, image artifacts or degrading effects may occur. The magnitude of these effects will be evaluated in a phantom study. 
a

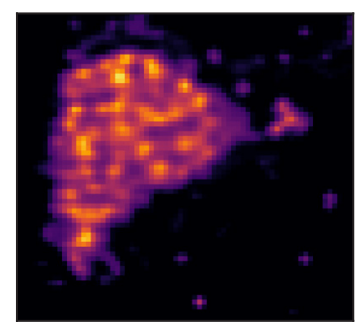

d

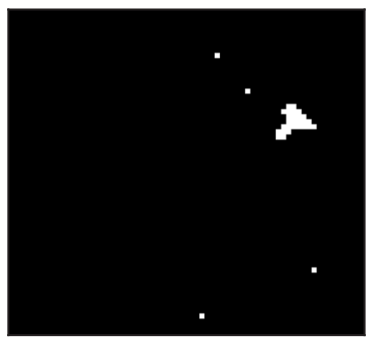

b

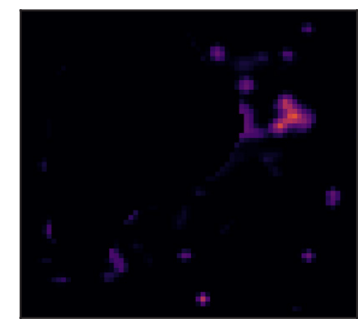

e
C

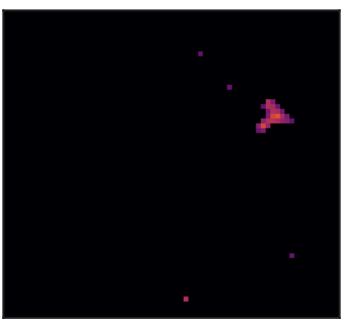

Figure 1: An illustration of the automatic process for detecting potential extrahepatic depositions. (a) The initial reconstructed image. (b) The lungs and liver are removed from the reconstruction. (c) The concentration threshold is applied. (d) The remaining voxels with activity are merged into clusters. (e) The threshold for the total activity present in a deposition is applied.

\section{Methods}

\section{Extrahepatic deposition detection}

To distinguish an extrahepatic deposition from a noisy background, a more or less arbitrary elevation of the reconstruction voxel activity and shape is normally assessed by the physician. Our proposed method is an automatic detection approach which we base on work originating from our institute with the same acquisition and reconstruction parameters as in this study [3].

The process of detecting potential extrahepatic depositions is illustrated in Fig. 1. From the initially reconstructed image (Fig. 1a), only the background is selected by removing the lung and liver masks (dilated by $2 \mathrm{~cm}$ ) from the image (Fig. 1b). These masks are expected to be known in advance. A threshold on the activity concentration (the voxel concentration must be greater than $0.017 \%$ of the total injected activity per $\mathrm{mL}$, as is the lowest concentration observed in an extrahepatic deposition [3]) is hence applied (Fig. 1c). The remaining voxels with activity are merged into clusters (Fig. 1d). The total activity in every cluster is hence measured and the clusters which have an activity greater than $0.1 \%$ of the total reconstructed activity (the lowest activity observed in an extrahepatic deposition [3]) are considered to be an extrahepatic deposition (Fig. 1e). The entire process is performed in 3D. 
Table 1: The studied configurations of the XCAT phantom in which one parameter was changed at a time in relation to the reference phantom. The reference phantom was created to be representative of clinical cases [3].

\begin{tabular}{l|ccc} 
& Concentration $[\mathrm{MBq} / \mathrm{mL}]$ & Volume $[\mathrm{mL}]$ & Location shift $[\mathrm{cm}]$ \\
\hline Reference & 0.10 & 15.19 & 0.0 \\
\hline Concentration changes & 0.05 & 15.19 & 0.0 \\
& 0.20 & 15.19 & 0.0 \\
& 0.50 & 15.19 & 0.0 \\
\hline Volume changes & 0.10 & 4.79 & 0.0 \\
& 0.10 & 7.27 & 0.0 \\
& 0.10 & 30.44 & 0.0 \\
\hline Location shifts & 0.10 & 15.19 & 1.95 \\
& 0.10 & 15.19 & 3.90 \\
& 0.10 & 15.19 & 7.79
\end{tabular}

\section{Scan duration algorithm}

The adaptive scan protocol is done by performing fast detector rotations of one minute each. The reconstruction is updated with the newly collected counts after every rotation. The following two criteria are evaluated for every intermediate reconstruction and the scan is terminated when one of them is met:

1. The total reconstructed activity in the background mask is lower than $0.075 \%$ of the total reconstructed activity.

The rationale behind the first criterion is that there cannot be an extrahepatic deposition if the activity in the background is negligible with respect to the total activity. The threshold of $0.075 \%$ was chosen because this is the defined summed extrahepatic activity threshold $(0.1 \%)$ minus a margin to account for noise fluctuations.

2. The relative difference in the mask of a detected extrahepatic deposition between two subsequent rotations (measured by calculating the total number of changed voxels between the two masks (i.e. from being part of the mask to being left out; or vice versa) and divided by the total number of voxels in the most recent mask) is lower than $5 \%$.

The rationale behind the second criterion is that the reconstruction will change substantially in the first few rotations because noise has a major impact. As the rotations progress, however, the reconstruction will become increasingly stable and hence the difference between two rotations will become increasingly smaller. The rate of this convergence is expected to depend on the activity distribution characteristics and will differ in every scan. We believe that, in clinical practice, it is most important to be confident in the shape and the location of an extrahepatic deposition so that its origin can be accurately determined. Hence, we will track the mask of the detected extrahepatic deposition over time to serve as a surrogate measure for these properties. The threshold of this extrahepatic mask change is a trade-off between accuracy and termination speed; we used 5\% because with this setting all extrahepatic depositions were accurately detected in the patient study. 


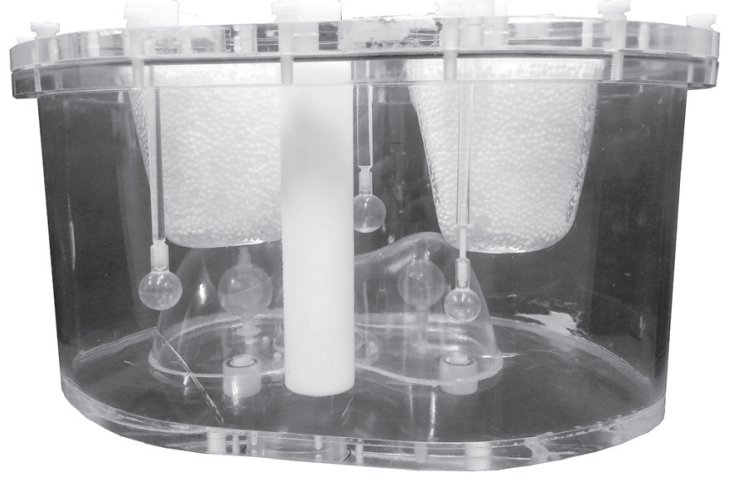

Figure 2: The anthropomorphic phantom with liver and lung compartments, and three extrahepatic depositions placed in the background compartment.

\section{Simulation study}

A simulation study was performed to study the activity distribution characteristics that influence the scan termination times. The XCAT phantom program [4] was used to simulate a patient distribution. The liver and lungs were uniformly filled with the LSF set to 5.0\% (such fractions are often encountered [5]). A spherical extrahepatic deposition was positioned close to the stomach (a common location for extrahepatic depositions [3]). The total activity in the phantom was fixed at $150 \mathrm{MBq}{ }^{99 \mathrm{~m}} \mathrm{Tc}$ (the currently injected activity at our institute [1]).

We varied the extrahepatic deposition's activity concentration, volume, and location (by moving the extrahepatic deposition downward over various distances from the reference location). Only one parameter was changed at a time (see Tab. 1 for all studied options). The reference phantom had an extrahepatic deposition activity concentration of $0.10 \mathrm{MBq} / \mathrm{mL}$, an extrahepatic deposition volume of $15.19 \mathrm{~mL}$, and its extrahepatic deposition positioned at the reference location. Such phantom configurations are representative of clinical cases [3].

The phantom was configured on a $128 \times 128 \times 128$ grid with a $3.9 \mathrm{~mm}$ isotropic voxel size. The nuclear projections were simulated with the Utrecht Monte Carlo System (UMCS) [6-7], which includes realistic physics for photon interactions in the patient and detector. The photopeak window was set to $129-150 \mathrm{keV}$ and the scatter window to $108-129 \mathrm{keV}$. The projector simulated a dual-head scanner with a step-and-shoot body-tracing orbit (with a $1 \mathrm{~cm}$ patient-detector gap), a low-energy high-resolution (LEHR) collimator, and 120 acquisition angles over 360 degrees.

Rotations of one minute each were simulated by the addition of Poisson noise (scaled to the total phantom activity) and background noise (obtained from a scan with no activity present) to the projections. For every phantom configuration, 20 rotations were evaluated by consecutively adding new counts to the projections.

The reconstructions were made with UMCS using the OSEM reconstruction algorithm with 6 iterations and 8 subsets, dual-energy window scatter correction $(k=0.5)$, and a post-reconstruction 
Table 2: The configuration of the phantom experiment. The background volume had no activity present.

\begin{tabular}{l|ccc} 
& Concentration $[\mathrm{MBq} / \mathrm{mL}]$ & Volume $[\mathrm{mL}]$ & Activity $[\mathrm{MBq}]$ \\
\hline Liver & 0.098 & 1172 & 115 \\
Lungs & 0.012 & 690 & 8 \\
Hot sphere & 0.78 & 15.9 & 12.4 \\
Hot sphere with cold core & 0.78 & 18.9 & 14.8 \\
Extrahepatic deposition 1 & 0.28 & 2.0 & 0.56 \\
Extrahepatic deposition 2 & 0.28 & 4.2 & 1.18 \\
Extrahepatic deposition 3 & 0.28 & 8.2 & 2.30
\end{tabular}

Gaussian filter of $5 \mathrm{~mm}$ full width at half maximum (FWHM). Attenuation correction was performed with the $\mu$-map obtained from the low-dose CT. These reconstruction parameters are the current clinical protocol in our institute.

The scan duration algorithm was then used to determine the optimal scan termination times for every phantom configuration. The mask and the activity of the detected extrahepatic deposition were determined in the reconstruction at termination time and the reconstruction at 20 minutes, and their differences were calculated. For every phantom configuration, 10 noise realizations were performed to study the stability of the results.

\section{Phantom study}

A phantom study was performed to evaluate the performance of adaptive scanning for physical scanner measurements and to study the influence of the fast continuous detector motion. An anthropomorphic phantom (IEL, Chilcompton, UK, Model ECT/TOR/P) was altered by the inclusion of three extrahepatic depositions (see Fig. 2). A hot sphere and hot sphere with a cold core were present in the liver compartment. The lungs were filled with an LSF of 5.2\% and the total activity in the phantom was $154 \mathrm{MBq}$. The deposition-to-liver activity concentration ratio was 2.86 . The individual activity levels can be found in Tab. 2.

Scanning was done on a Siemens Symbia T16 system by performing 20 rotations of one minute each in continuous motion. The acquisition and reconstruction parameters were (except for the fast continuous detector motion) the same as in the simulation study. The scan duration algorithm was used to determine the optimal scan termination times and the extrahepatic mask and activity differences with the 20-minute scan were measured.

Some resolution degradation is expected when scanning with fast continuous detector motion because the detector orbit can fluctuate over the rotations and the detector-patient distance and the detector rotation angle can fluctuate within one bin. To evaluate the magnitude of the detector orbit fluctuations, the detector distance was measured over the 20 rotations and its standard deviation was calculated. To evaluate the influence of the continuous motion, the phantom was also scanned with a single-rotation step-and-shoot 20-minute scan. The reconstruction of this scan was visually and quantitatively compared to the reconstruction of 20 one-minute rotations. 
a
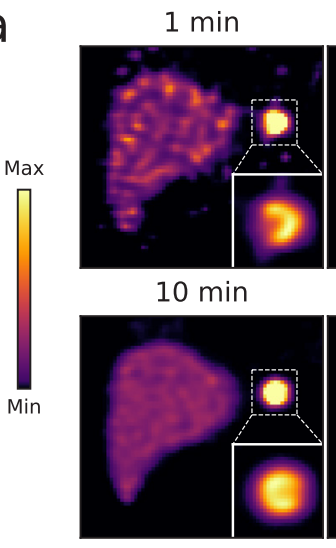

$5 \mathrm{~min}$

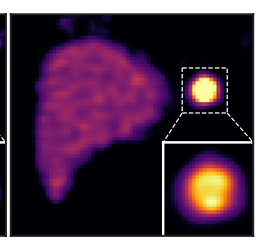

$20 \mathrm{~min}$

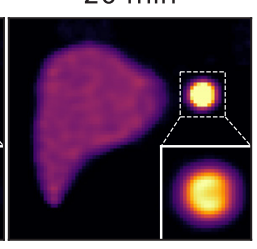

b

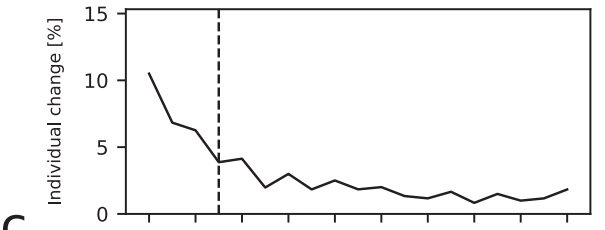

C

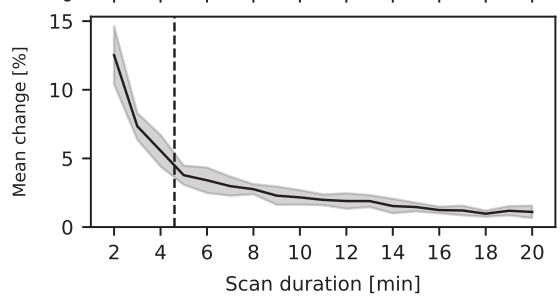

Figure 3: (a) Slices from the reconstructions for four scan durations for the XCAT phantom with $0.50 \mathrm{MBq} / \mathrm{mL}$ extrahepatic deposition activity concentration. The image maximum was set to $5 \mathrm{x}$ the liver concentration. (b) The relative change in the mask of the extrahepatic deposition, as a function of scan duration. The black dashed line indicates the scan termination time. (c) The mean relative change in the mask for 10 (individually analyzed) noise realizations. The shaded error bars indicate the standard deviation. The black dashed line indicates the mean scan termination time.

\section{Patient study}

The potential for adaptive scanning was then evaluated for clinical patient activity distributions. 129 patient projections with corresponding attenuation maps and body, lung, and liver delineations were used. All patients received an injection of approximately $150 \mathrm{MBq}{ }^{99 \mathrm{~m}} \mathrm{Tc}-\mathrm{MAA}(0.8 \mathrm{mg}$, Technescan LyoMAA) in accordance with the radioembolization guidelines [8]. The acquisition and reconstruction parameters were the same as in the simulation study.

Since the patient scans were performed with a single 20 -minute rotation, 20 one-minute rotations were created by subsampling uniform random subsets from the 20-minute projection: all counts of the 20-minute projection were considered individual events and their positions ( $x, y$, angle) were put in an event list-mode. This list-mode was shuffled randomly and the first (or second, etc.) $5 \%$ of this new list-mode was used to create the 20 one-minute minute acquisitions. The sampling process was repeated 10 times to study the stability of the reconstructions to different noise distributions.

The scan duration algorithm was used to determine the optimal scan termination time for every activity distribution. The extrahepatic deposition mask and activity differences with the 20-minute scan were measured. For every detected extrahepatic deposition, the contrast-to-noise ratio was calculated by dividing the contrast between the extrahepatic deposition mask and the background mask by the standard deviation of the background mask. The background mask was available from the automatic extrahepatic deposition detection methodology and consisted of the body contour from which the liver, lung, and all detected extrahepatic deposition masks were subtracted. The analyses were done for the 10 noise realizations separately. 

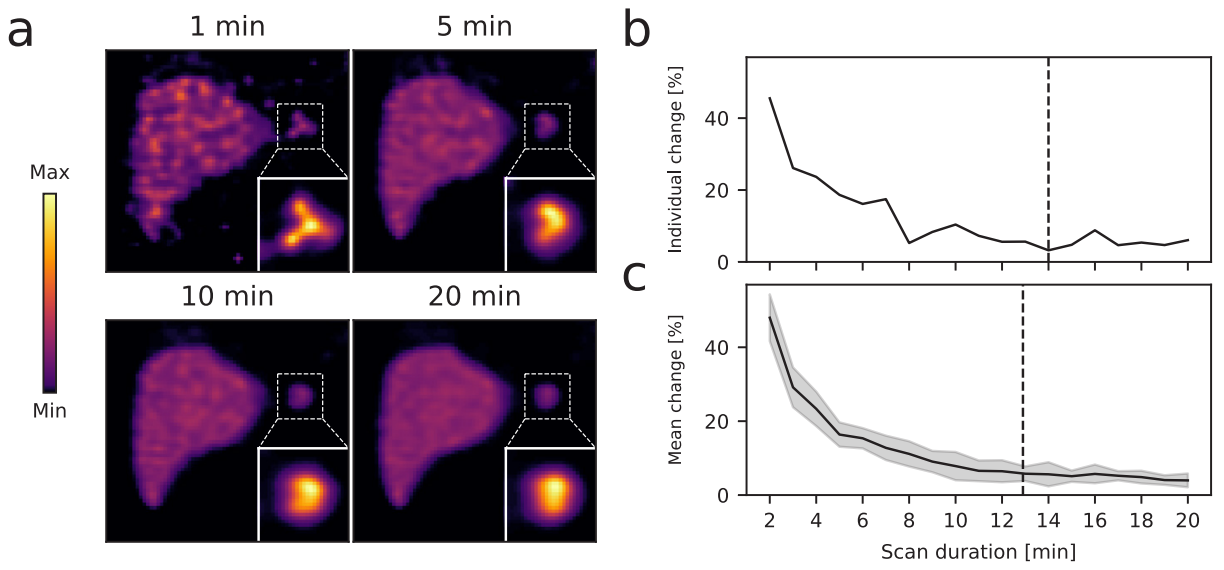

Figure 4: (a) Slices from the reconstructions for four scan durations for the XCAT phantom with $0.05 \mathrm{MBq} / \mathrm{mL}$ extrahepatic deposition activity concentration. The image maximum was set to $5 \mathrm{x}$ the liver concentration. (b) The relative change in the mask of the extrahepatic deposition, as a function of scan duration. The black dashed line indicates the scan termination time. (c) The mean relative change in the mask for 10 (individually analyzed) noise realizations. The shaded error bars indicate the standard deviation. The black dashed line indicates the mean scan termination time.

For comparison, the scan duration was also shortened without separate optimization for every patient activity distribution (i.e. by scanning with a single rotation with the same shortened scan duration for all patients). The scan duration was gradually decreased and it was determined up until which scan duration all extrahepatic depositions were accurately detected. An extrahepatic deposition was considered to be accurately detected when the automatic detection algorithm retrieved the same number of extrahepatic depositions as in the 20-minute scan and for those masks, the difference in their center of mass was lower than 5 pixels.

\section{Results}

\section{Simulation study}

Examples of the reconstructions obtained from the XCAT phantom with an extrahepatic deposition activity concentration of $0.50 \mathrm{MBq} / \mathrm{mL}$ are shown in Fig. 3a. Some differences are observed in the extrahepatic deposition shape and size between the 1 and 5 -minute scans. After 5 minutes, however, almost no changes are observed.

The relative change in the mask of the extrahepatic deposition is shown in Fig. $3 \mathrm{~b}$ (for the scan of Fig. 3a). The relative change dropped below $5 \%$ for a scan duration of 5 minutes, which is in agreement with the visual observation from Fig. 3a. The mean mask changes of the 10 (individually analyzed) noise realizations are shown in Fig. $3 c$. The mean scan termination time was $4.6 \pm 0.5$ min, which shows that the proposed algorithm provides (for this phantom configuration) relatively stable results. 
Table 3: The scan termination times of the studied extrahepatic deposition configurations. The difference in the extrahepatic deposition mask and the extrahepatic activity compares the scan with its duration from adaptive scanning with the 20-minute scan. The standard deviations were retrieved from the 10 (individually analyzed) noise realizations.

\begin{tabular}{lc|ccc} 
& & Scan termination $[\mathrm{min}]$ & Mask difference [\%] & Activity difference [\%] \\
\hline Concentration $[\mathrm{MBq} / \mathrm{mL}]$ & 0.05 & $12.9 \pm 2.8$ & $11.9 \pm 3.3$ & $4.0 \pm 1.6$ \\
& 0.10 & $8.8 \pm 1.7$ & $9.7 \pm 2.1$ & $2.0 \pm 1.2$ \\
0.20 & $7.3 \pm 0.9$ & $9.3 \pm 1.7$ & $1.4 \pm 1.4$ \\
0.50 & $4.6 \pm 0.5$ & $7.7 \pm 1.0$ & $1.5 \pm 1.0$ \\
& & & & \\
Volume $[\mathrm{mL}]$ & 4.79 & $9.3 \pm 3.1$ & $15.8 \pm 6.8$ & $2.3 \pm 2.8$ \\
& 7.27 & $9.1 \pm 1.6$ & $9.6 \pm 3.3$ & $2.0 \pm 1.2$ \\
& 15.19 & $8.8 \pm 1.7$ & $9.7 \pm 2.1$ & $0.8 \pm 0.7$ \\
& 30.44 & $7.9 \pm 0.9$ & $8.5 \pm 2.3$ & \\
& & & & $2.0 \pm 1.2$ \\
Location shift $[\mathrm{cm}]$ & 0.00 & $8.8 \pm 1.7$ & $9.7 \pm 2.1$ & $1.5 \pm 1.1$ \\
& 1.95 & $8.2 \pm 1.7$ & $9.0 \pm 2.4$ & $3.0 \pm 1.6$ \\
& 3.90 & $6.7 \pm 0.8$ & $11.8 \pm 2.7$ & $2.8 \pm 1.8$
\end{tabular}

The same evaluation as described above was performed on the phantom with $0.05 \mathrm{MBq} / \mathrm{mL}$ (instead of $0.50 \mathrm{MBq} / \mathrm{mL}$ as above). Slices from the obtained reconstructions are shown in Fig. 4a. Visually, the extrahepatic deposition changed considerably more over the scan duration. This behavior is also found in the graphs on the relative change in the mask (Fig. $4 \mathrm{~b}$ and c): the phantom configuration reached the $5 \%$ threshold level after $12.9 \pm 2.8 \mathrm{~min}$, which is substantially longer than in the previous phantom configuration. This phantom comparison illustrates that the activity distribution characteristics have a substantial influence on the scan termination time.

The scan termination times and the corresponding differences in the masks (compared with the 20-minute scan) are shown in Tab. 3 for all studied phantom configurations. The predominant factor for the scan termination time was the activity concentration of the extrahepatic deposition: depositions with a higher activity concentration terminated earlier. The volume of the extrahepatic deposition had some influence: larger extrahepatic depositions terminated earlier. The location of the extrahepatic deposition showed no substantial influence.

\section{Phantom study}

The mean detector orbit of the 20 rotations in the phantom study is shown together with the corresponding standard deviation in Fig. 5. The standard deviation was small in relation to the detector distance mean, which indicates that the detector orbit was able to reproduce well over the multiple rotations. 


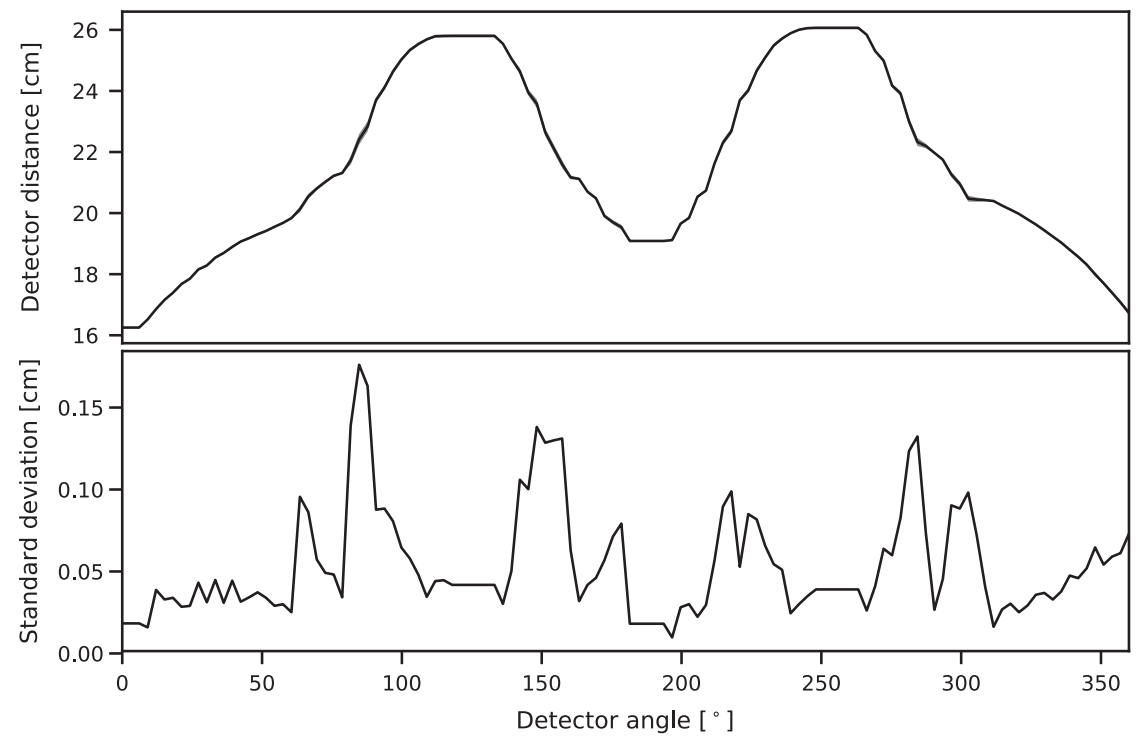

Figure 5: The mean and the standard deviation of the detector distance as a function of the detector angle for the 20 one-minute scans in continuous motion.

Slices from the reconstructions of the phantom experiment with varying scan durations are shown in Fig. 6a. There is a substantial change in the shape of the extrahepatic deposition between the scan of 1 and 5 minutes in duration. However, between 5 and 20 minutes of scan duration, hardly any differences can be observed.

This behavior is also found in the graphs on the relative change in the extrahepatic deposition mask in Fig. 6b, which show that the 5\% threshold level was reached after 4, 4, and 6 minutes for the small, medium, and large extrahepatic depositions, respectively. After 6 minutes of scanning, the extrahepatic mask differences with the 20 -minute scan were $8.1 \%, 4.4 \%$, and $8.2 \%$ and the activity differences were $4.7 \%, 2.3 \%$, and $1.2 \%$ for the small, medium, and large extrahepatic depositions, respectively. This indicates that, also for the phantom experiment, the scan might have been earlier terminated.

The reconstruction of the single-rotation 20-minute scan is shown in Fig. 6c. It can be observed that the reconstruction from the single-rotation is somewhat sharper than the one obtained with multiple fast rotations: the intrahepatic deposition is more uniform and the deposition with cold core is slightly more pronounced. The contrast-to-noise ratio (CNR) of the intrahepatic deposition was 38.4 for the single-rotation 20-minute scan and 35.4 for the one obtained with multiple fast rotations. We conclude that fast detector motion gives rise to some resolution degradation, but will ultimately not be limiting the feasibility of adaptive scanning. 

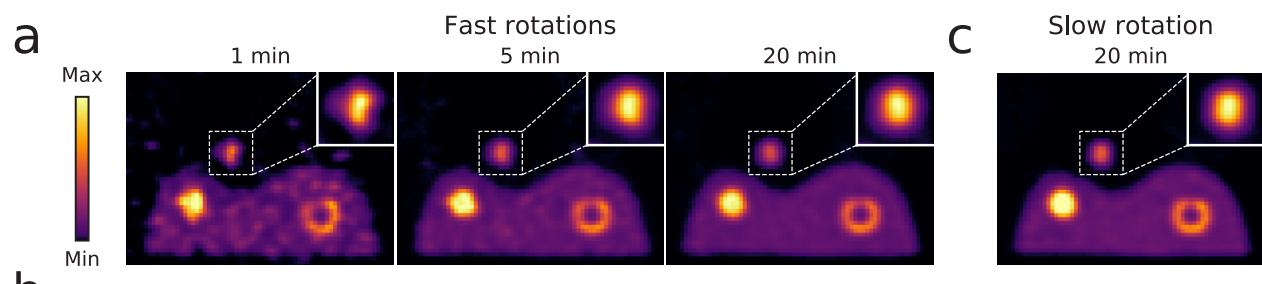

b

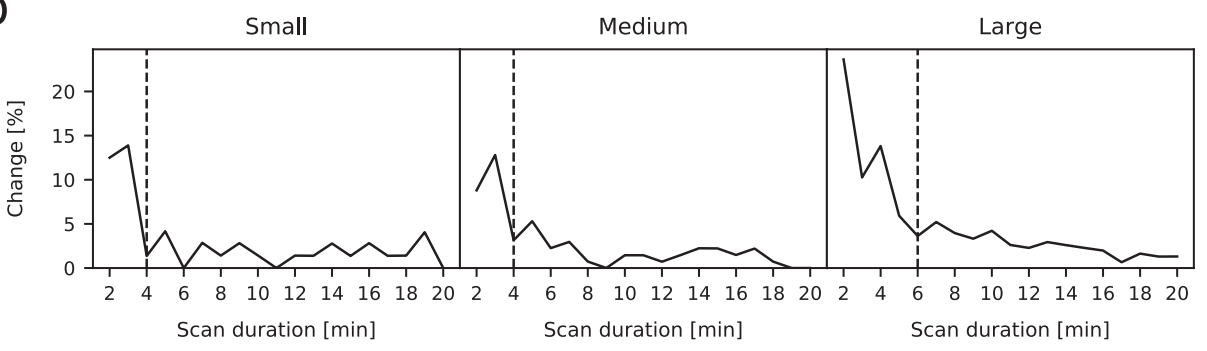

Figure 6: (a) Slices from the reconstructions (shown is the slice with the large extrahepatic deposition) of the phantom experiment obtained from three scan durations with fast rotations and (c) from a slow full-duration scan. (b) The relative change in the extrahepatic deposition mask, as a function of scan duration. The black dashed lines indicate the scan durations in which the $5 \%$ change between two subsequent rotations was achieved.

\section{Patient study}

An example of a patient activity distribution with a high-concentration extrahepatic deposition is shown in Fig. 7. Evident is that the extrahepatic deposition barely changes in shape and size over time, which is also illustrated by the graphs on the relative change in the extrahepatic deposition mask. The mean scan termination time for this activity distribution was $5.7 \pm 1.1 \mathrm{~min}$. This shows that also for a more complex activity distribution (i.e. not spherical depositions as in the simulation and phantom studies), the scan might have been earlier terminated.

An example of a patient activity distribution with a low-concentration extrahepatic deposition is shown in Fig. 8. For this activity distribution, the shape of the extrahepatic deposition does change considerably over time. The mean scan termination time for this activity distribution was $16.6 \pm 3.4$ min. This illustrates that large differences in the scan termination times can be expected for patient activity distributions.

The collection of scan termination times for all patient scans (for a single noise realization) is shown in Fig. 9a. A large fraction of the activity distributions was already terminated at one minute; these are the activity distributions that had $<0.075 \%$ total extrahepatic activity after one rotation. A substantial fraction of the activity distributions did not converge at 20 minutes; these scans might have benefited from a longer scan duration.

For the 129 activity distributions, 28 extrahepatic depositions were detected in 25 patients for the 20-minute scans. Adaptive scanning accurately detected all depositions in all noise realizations 

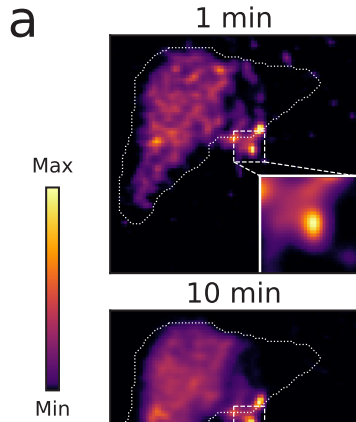

$10 \min$

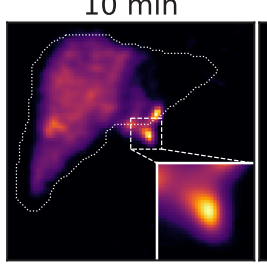

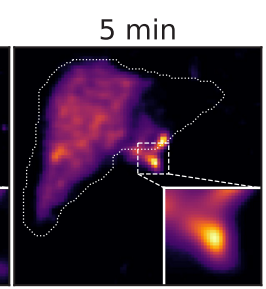

$20 \min$

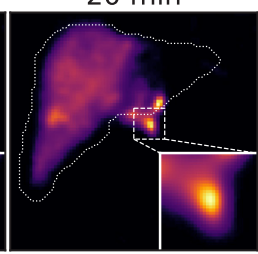

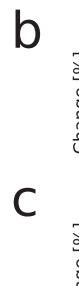
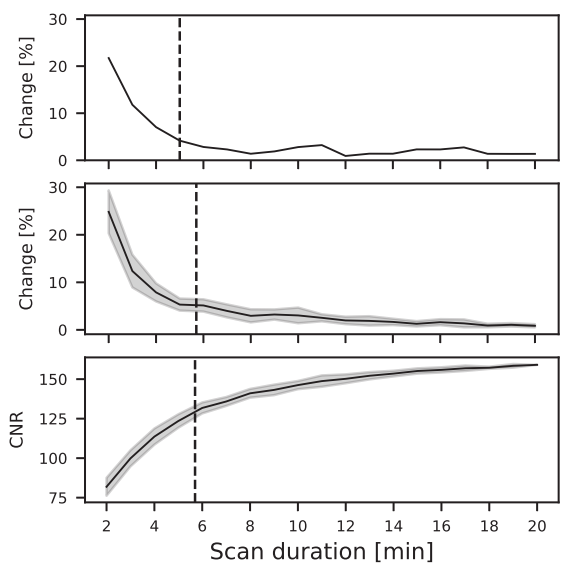

Figure 7: (a) Slices from the reconstructions for four scan durations for a patient activity distribution with a high-concentration extrahepatic deposition. (b) The relative change in the mask of the extrahepatic deposition, as a function of scan duration. The black dashed line indicates the scan termination time. (c) The mean relative change in the extrahepatic deposition mask for 10 (individually analyzed) noise realizations. The shaded error bars indicate the standard deviation. The black dashed line indicates the mean scan termination time. (d) The mean contrast-to-noise ratio for 10 (individually analyzed) noise realizations. The shaded error bars indicate the standard deviation. The black dashed line indicates the mean scan termination time.

(using the 20-minute scans as the ground truth). The contrast-to-noise ratios of the detected extrahepatic depositions at the scan termination times are shown (for a single noise realization) in Fig. 9b. All extrahepatic depositions in all noise realizations had a contrast-to-noise ratio $>4$ and hence should also be visually detectable [9].

The mean scan durations and the mask and activity differences of the extrahepatic depositions at the scan termination times with the 20-minute scan are shown in Tab. 4 for the individual noise realizations. The mean scan duration was $4.8 \pm 0.2$ minutes, the mean difference in the mask with the full-duration scan was $7.0 \pm 1.0 \%$, and the mean activity difference was $2.0 \pm 0.5 \%$.

The minimum scan durations (with a single-rotation) required for accurate detection of all extrahepatic depositions (using the same detection criteria as with adaptive scanning) are also shown in Tab. 4. Several noise realizations could not be shortened: i.e. they require the full 20-minute scan for accurate detection. In the remaining noise realizations, a scan shortening of only a few minutes could be achieved. The mean minimum scan duration required for scanning with a single-rotation was $18.8 \pm 1.1$ minutes.

In conclusion, the switch from a single-rotation scan to an adaptive scanning protocol allows the mean scan duration (for the studied patient distributions) to be reduced from $18.8 \pm 1.1$ minutes to $4.8 \pm 0.2$ minutes, without compromising the detectability of extrahepatic depositions. 

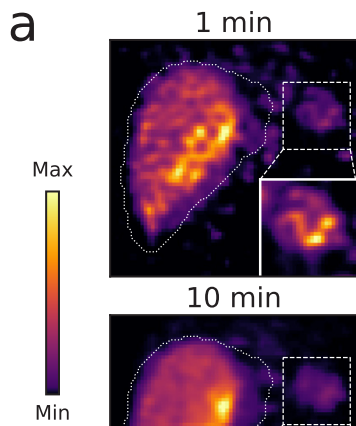

$10 \mathrm{~min}$

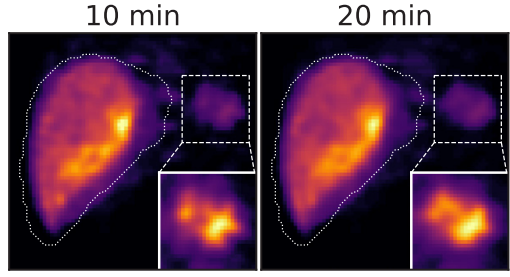

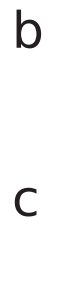

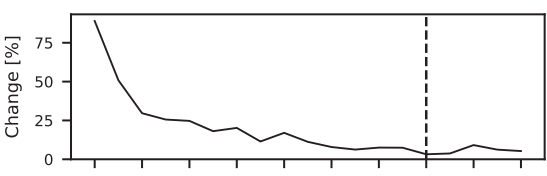

C
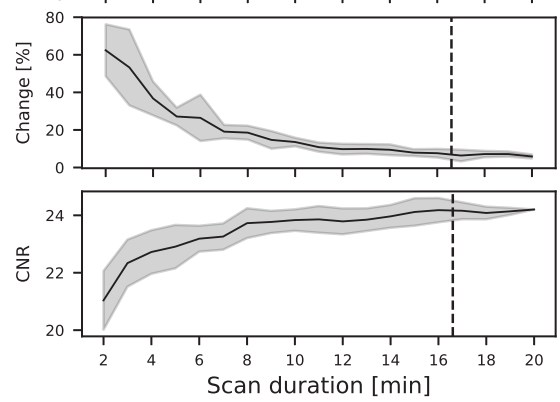

Figure 8: (a) Slices from the reconstructions for four scan durations for a patient activity distribution with a low-concentration extrahepatic deposition. (b) The relative change in the mask of the extrahepatic deposition, as a function of scan duration. The black dashed line indicates the scan termination time. (c) The mean relative change in the extrahepatic deposition mask for (individually analyzed) 10 noise realizations. The shaded error bars indicate the standard deviation. The black dashed line indicates the mean scan termination time. (d) The mean contrast-to-noise ratio for 10 (individually analyzed) noise realizations. The shaded error bars indicate the standard deviation. The black dashed line indicates the mean scan termination time.

\section{Discussion}

We investigated the potential for an adaptive scan duration protocol in SPECT for the radioembolization pretreatment procedure and showed the feasibility of terminating scans at an earlier point in time without affecting the detectability of extrahepatic depositions.

We believe that our approach is not limited to the detection of extrahepatic depositions. As long as a measure of interest is available, adaptive scanning can be performed to shorten the scan duration. For instance, we envision that it could be used in cardiac SPECT since quantitative measures are often evaluated in this protocol. However, we acknowledge that some protocols do not have clearly defined objectives and adaptive scanning would be not be possible for these cases.

We also acknowledge that the scan duration range that can be achieved in clinical practice is limited. The workup of a patient (positioning, providing instructions, etc.) requires several minutes and would become the limiting factor for very fast (one-minute) scans. On the other end, patients can only lie still for a certain amount of time (usually approximately 30 minutes) and will start moving for longer scans. It may be beneficial to set a minimum and maximum scan duration to ensure that such practical considerations do not interfere with the scanning workflow.

Changes would need to be made to the patient and technician scheduling if adaptive scanning is employed in clinical practice since the scan start and finish times are no longer known in advance. Rather than scanning a patient on a specific scanner at a specific time, the patient would be scanned 

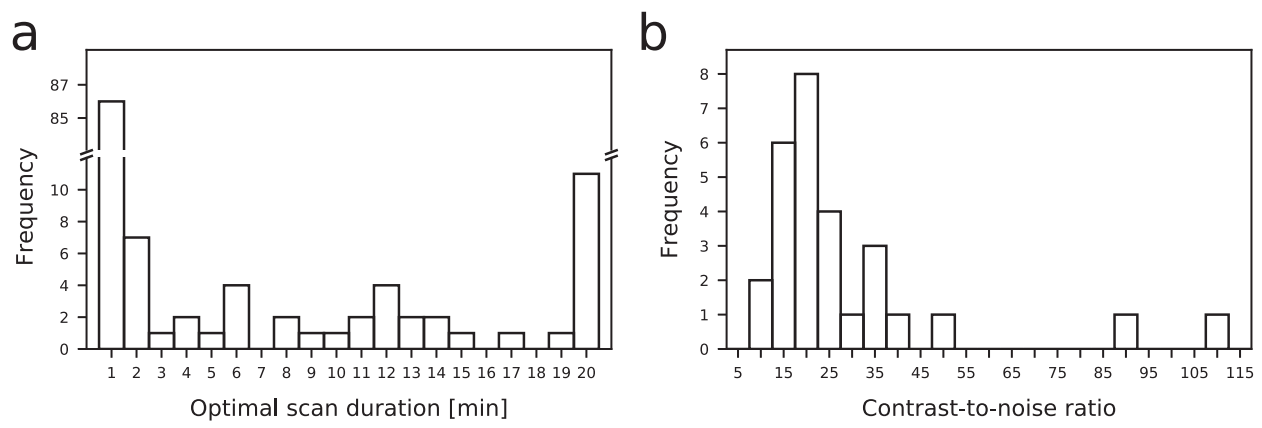

Figure 9: (a) The scan termination times for the 129 patient activity distributions for one noise realization. (b) The contrast-to-noise ratios of the extrahepatic depositions at the scan termination times for one noise realization.

in a certain timeslot on whichever scanner is first available. This form of scheduling becomes increasingly practical when more scanners are available since scan duration extremities would have less impact on the total workflow.

A specific case that does not require scheduling changes would be the use of adaptive scanning in interventional SPECT. Our group is developing a mobile, compact SPECT scanner that can be used in the intervention room [10-12]. We envision that this system could be used to perform a SPECT scan after every ${ }^{99 \mathrm{~m}} \mathrm{Tc}-\mathrm{MAA}$ injection in the pretreatment procedure of radioembolization so that the response to every injection is individually monitored. For this purpose, adaptive scanning would be beneficial in minimizing the total time spent in the intervention room.

The method in this work has been developed only for the detection of extrahepatic depositions since this is the most common objective for the pretreatment procedure of radioembolization.

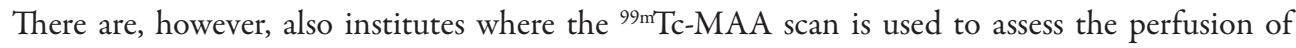
target lesions (with potential necrotic cores) and the activity to the parenchyma. Furthermore, the potential for the ${ }^{99 \mathrm{~m}} \mathrm{Tc}-\mathrm{MAA}$ scan to be used for dosimetry planning is being investigated within trials $[13,14]$. Our algorithm does not take such (intrahepatic) measures into account and hence should be modified if the above methods are introduced in regular clinical practice.

A pre-requisite for adaptive scanning to function is that the reconstructions are performed in a fast manner. The current clinical reconstruction software takes approximately a minute to finish, which is likely sufficient for use in clinical practice. Further speed-up may, for instance, be achieved by employing GPU-based software [15] or deep learning approaches [16].

A further requirement for adaptive scanning to be used in the pretreatment procedure of radioembolization is that the liver and lung delineations need to be present in advance. The lung delineations can be normally be created quickly via thresholding of the Hounsfield units of the lowdose CT. For the liver delineation, atlas-based methods are often employed. Since the liver and lung delineations are dilated by the relatively large margin of $2 \mathrm{~cm}$ to cope with partial volume effects and respiratory motion, small dilatation errors are not expected to substantially alter the results. 
Table 4: For adaptive scanning, shown are the mean scan duration and the difference in the extrahepatic deposition mask and the extrahepatic activity with the 20-minute scan. For scanning with a single-rotation, shown is the minimum scan duration required for accurate detection of all extrahepatic depositions.

\begin{tabular}{c|ccc|c} 
& \multicolumn{3}{|c|}{ Adaptive scanning } & Single-rotation \\
\hline $\begin{array}{c}\text { Noise } \\
\text { realization }\end{array}$ & $\begin{array}{c}\text { Mean scan } \\
\text { duration [min] }\end{array}$ & $\begin{array}{c}\text { Mean mask } \\
\text { difference [\%] }\end{array}$ & $\begin{array}{c}\text { Mean activity } \\
\text { difference [\%] }\end{array}$ & $\begin{array}{c}\text { Minimum required } \\
\text { scan duration [min] }\end{array}$ \\
\hline 1 & 4.7 & 5.8 & 1.9 & 20 \\
2 & 4.4 & 8.7 & 2.7 & 20 \\
3 & 4.8 & 6.2 & 2.0 & 18 \\
4 & 5.1 & 5.7 & 2.2 & 18 \\
5 & 4.8 & 6.8 & 1.1 & 18 \\
6 & 4.9 & 7.8 & 2.0 & 20 \\
7 & 5.0 & 6.5 & 1.5 & 20 \\
8 & 4.8 & 7.9 & 2.7 & 17 \\
9 & 4.8 & 6.3 & 1.3 & 18 \\
10 & 4.8 & 8.0 & 2.4 & $18.8 \pm 1.1$
\end{tabular}

It was shown that fast scanner rotation resulted in a slightly decreased reconstruction resolution. In clinical practice, however, patient motion is expected to be the major limiting factor for resolution and it is hence not expected that severe problems would arise from the fast detector rotation. If it does prove that the fast detector rotation is undesired, the rotation speed could simply be lowered somewhat (by e.g. making rotations of 2 minutes each).

The threshold value for the relative change in the mask to consider the scan converged is linked to the objective that one wants to achieve. A lower threshold provides reconstructions with a higher confidence on the imaging task, a higher threshold allows for faster scans. We chose the $5 \%$ threshold in this work because with this value all extrahepatic depositions in the patient scans were accurately detected. Eventually, it depends on the preference of the physician which extrahepatic depositions (e.g. which activity and volume) need to be detectable and which ones can be safely ignored.

Other methods for adaptive scanning have been previously developed (primarily in positronemission tomography (PET)) $[17,18]$. The majority of these methods are projection-based: i.e. the number of photons collected is measured in the first few projections in a certain region of interest, from which the optimal scan duration is then derived. We cannot use these methods for two reasons. First, it is unknown in advance where an extrahepatic deposition will arise and hence it is not possible to focus on a certain region of interest beforehand. Second, due to the limited intrinsic spatial resolution and the substantial scatter contribution of the SPECT projections, it is challenging to detect small activity depositions. For these reasons, we believe that the reconstructionbased method as described in this work will outperform projection-based ones.

The relationships between the extrahepatic deposition characteristics (size, activity concentration, location) and the resulting optimal scan duration were only studied in the simulation study since 
the sample size of the patient distributions was too low to obtain statistically significant results. It is interesting to revisit the relationships when more patient data becomes available.

The optimal scan durations obtained in the patient study showed that for a substantial fraction of the distributions, the scans could have been terminated after one minute without affecting the extrahepatic detection accuracy. The study of Van der Velden et al. [2] showed that the LSF estimation has an error of less than 0.5 percent point with the ground truth of $5 \%$ for a one minute scan. The LSF estimation is hence expected to be sufficiently accurate for use in clinical practice, even for the very short scans as studied in this work.

In our institute, only one SPECT scan is made per radioembolization procedure since the field of view of the detectors is sufficiently large to cover all potential extrahepatic deposition origins. If one, however, wants to visualize multiple body regions (e.g. because of a smaller detector coverage or a large patient), more acquisitions can be made. These scans can be performed with the same methodology as described in this work and should be separately analyzed.

The approach in this work investigated a scanner with a rotating detector because such scanners are most used in current clinical practice. SPECT scanners with static detectors are, however, becoming increasingly available (e.g. the D-SPECT for cardiac imaging or the VERITON for full-body imaging; both developed by Spectrum Dynamics Medical). Such devices could similarly benefit from adaptive scanning by evaluating the list-mode acquisition e.g. every minute and are furthermore not constrained by the scanner mechanics.

\section{Conclusion}

The feasibility of an adaptive SPECT scan duration (by assessing the activity distribution characteristics during scanning) was demonstrated for the detection of extrahepatic depositions in the pretreatment procedure of radioembolization. We believe that our approach could also benefit other forms of SPECT scanning, as long as a measure of interest is available for optimization.

\section{References}

1. Braat AJAT, Smits MLJ, Braat MNGJA, et al. $\mathrm{Y}^{90}$ Hepatic Radioembolization: An Update on Current Practice and Recent Developments, J. Nucl. Med. 56(2):1079-1087 (2015)

2. Van der Velden S, Dietze MMA, Viergever MA, De Jong HWAM. Fast technetium-99m liver SPECT for evaluation of the pre-treatment procedure for radioembolisation dosimetry, Med. Phys. 46(1):345355 (2019)

3. Prince JF, Van Rooij R, Bol GH, De Jong HWAM, Van den Bosch MAAJ, Lam MGEH. Safety of a Scout Dose Preceding Hepatic Radioembolization with ${ }^{166}$ Ho Microspheres, J. Nucl. Med. 56(6):817-23 (2015)

4. Segars WP, Sturgeon G, Mendonca S, Grimes J, Tsui BMW. 4D XCAT phantom for multimodality imaging research, Med. Phys. 37(9):4902-15 (2010)

5. Xing M, Lahti S, Kokabi N, Schuster DM, Camacho JC, Kim HS. ${ }^{90}$ Y Radioembolization Lung Shunt Fraction in Primary and Metastatic Liver Cancer as a Biomarker for Survival, Clin. Nucl. Med. 41(1):21$7(2016)$ 
6. De Jong HWAM, Slijpen ETP, Beekman FJ. Acceleration of Monte Carlo SPECT simulation using convolution-based forced detection, IEEE Trans. Nucl. Sci. 48(1):58-64 (2001)

7. Dietze MMA, Van der Velden S, Lam MGEH, Viergever MA, De Jong HWAM. Fast quantitative reconstruction with focusing collimators for liver SPECT, EJNMMI Phys. 5(1):28 (2018)

8. Kennedy A, Nag S, Murthy R, et al. Recommendations for radioembolization of hepatic malignancies using yttrium-90 microsphere brachytherapy: a consensus panel report from the radioembolization brachytherapy oncology consortium, Int J Radiat Oncol Biol Phys 68(1):13-23 (2007)

9. Rose A. The sensitivity performance of the human eye on an absolute scale, J Opt Soc Am 38(2):196-208 (1948)

10. Van der Velden S, Kunnen B, Koppert WJC, et al. A Dual-layer Detector for Simultaneous Fluoroscopic and Nuclear Imaging, Radiology 290(3):833-838 (2019)

11. Dietze MMA, Kunnen B, Van der Velden S, Steenbergen LJH, Koppert WJC, Viergever MA, De Jong HWAM. Performance of a dual-layer scanner for hybrid SPECT/CBCT, Phys. Med. Biol. 64(10):105020 (2019)

12. Dietze MMA, Bastiaannet R, Kunnen B, Van der Velden S, Lam MGEH, Viergever MA, De Jong HWAM. Respiratory Motion Compensation in Interventional Liver SPECT using Simultaneous Fluoroscopic and Nuclear Imaging, Med. Phys. 46(8):3496-3507 (2019)

13. Center Eugene Marquis. Internal Radiation Therapy for Hepatocellular Carcinomas With Therasphere: Optimized Dosimetry Versus Standard Dosimetry, https:/clinicaltrials.gov/ct2/show/NCT02582034, Accessed on December 13, 2019

14. Chauhan N, Bukovcan J, Boucher E, et al. Intra-arterial TheraSphere yttrium-90 glass microspheres in the treatment of patients with unresectable hepatocellular carcinoma: Protocol for the STOP-HCC phase 3 randomized controlled trial, JMIR Res. Protoc. 7(8):e11234 (2018)

15. Rydén T, Heydorn Lagerlöf J, Hemmingsson J, et al. Fast GPU-based Monte Carlo code for SPECT/CT reconstructions generates improved 177Lu images. EJNMMI Phys. 5(1):1 (2018)

16. Dietze MMA, Branderhorst WJ, Kunnen B, Viergever MA, De Jong HWAM. Accelerated SPECT image reconstruction with FBP and an image enhancement convolutional neural network, EJNMMI Phys. 6(1):14 (2019)

17. Krizsan AK, Czernin J, Balkay L, Dahlbom M. Whole Body PET Imaging Using Variable Acquisition Times, IEEE Trans. Nucl. Sci. 61(1):115-20 (2014)

18. Namías M, Jeraj R. Patient and scanner-specific variable acquisition times for whole-body PET/CT imaging, Phys. Med. Biol. 64(20):205013 (2019) 


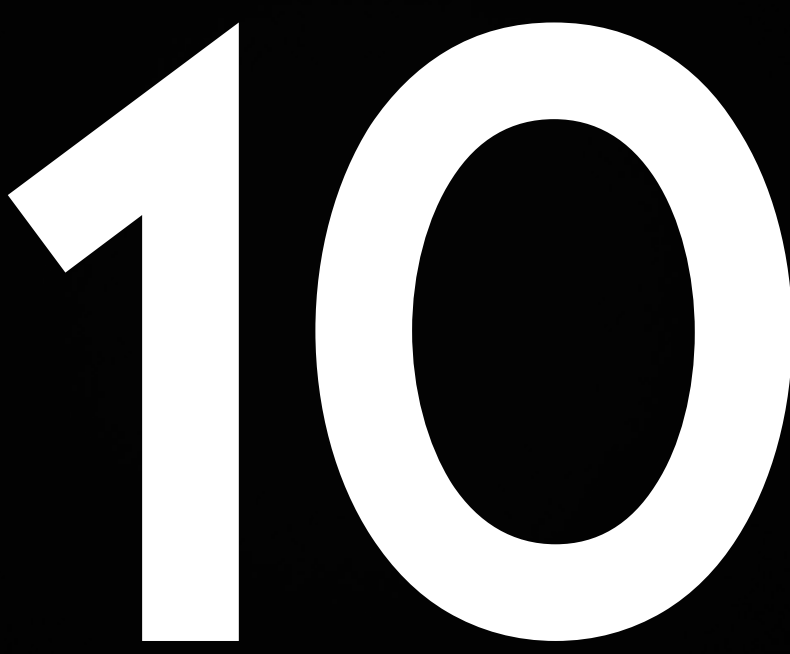


\section{ACCELERATED IMAGE RECONSTRUCTION WITH DEEP LEARNING}

PUBLISHED AS:

MMA Dietze, WJ Branderhorst, B Kunnen, MA Viergever, HWAM de Jong

Accelerated SPECT Image Reconstruction with FBP and an Image Enhancement Convolutional Neural Network 


\begin{abstract}
Background

Monte Carlo-based iterative reconstruction to correct for photon scatter and collimator effects has been proven to be superior over analytical correction schemes in single-photon emission computed tomography (SPECT/CT), but is currently not commonly used in daily clinical practice due to the long associated reconstruction times. We propose to use a convolutional neural network (CNN) to upgrade fast filtered back projection (FBP) image quality so that reconstructions comparable in quality to the Monte Carlo-based reconstruction can be obtained within seconds.
\end{abstract}

\title{
Results
}

A total of 128 technetium-99m macroaggregated albumin pre-treatment SPECT/CT scans used to guide hepatic radioembolisation were available. Four reconstruction methods were compared: FBP, clinical reconstruction, Monte Carlo-based reconstruction, and the neural network approach. The $\mathrm{CNN}$ generated reconstructions in $5 \mathrm{sec}$, whereas clinical reconstruction took $5 \mathrm{~min}$ and the Monte Carlo-based reconstruction took $19 \mathrm{~min}$. The mean squared error of the neural network approach in the validation set was between that of the Monte Carlo-based and clinical reconstruction and the lung shunting fraction difference was lower than 2 percent point. A phantom experiment showed that quantitative measures required in radioembolisation were accurately retrieved from the CNN generated reconstructions.

\section{Conclusions}

FBP with an image enhancement neural network provides SPECT reconstructions with quality close to that obtained with Monte Carlo-based reconstruction within seconds. 


\section{Background}

Monte Carlo-based iterative reconstruction to correct for scatter and collimator effects has been proven to be superior over more analytical correction schemes, albeit at the cost of reconstruction times of potentially several hours [1-3]. This has up to now limited introduction of such techniques into daily clinical practice because the requirement of reconstruction job queues causing interrupted workflow is considered bothersome. In addition, technologists prefer to quickly judge whether a scan was correctly performed, e.g. by validating whether a patient has moved during the examination. Substantial reconstruction acceleration can be achieved with code parallelization, the use of dedicated workstations, and restrictions on the matrix size or number of iterations, which might bring the reconstruction time to a clinically acceptable value. We will in this work, however, explore a different method for acceleration, that is also less dependent on reconstruction settings for its required reconstruction time.

Deep learning has emerged in recent years for a variety of applications in medical imaging, such as segmentation, super-resolution, and denoising [4]. We believe there is a major opportunity for these networks to aid single-photon emission computed tomography (SPECT) by accelerating the reconstruction step. Specifically, we propose to first generate a low-quality reconstruction using fast filtered back projection (FBP) and then pass the result to an image enhancement convolutional neural network. Such a trained network would improve reconstruction quality and would be able to generate its results within seconds.

Although our approach is valid for all forms of SPECT/CT imaging, the focus of this work will be on potential implementation in the hepatic radioembolisation workflow [5]. In this treatment, microspheres filled with radioactivity are inserted into the liver to deliver a damaging dose to the tumour. A scan of technetium-99m macroaggregated albumin ( $\left.{ }^{99 m} \mathrm{Tc}-\mathrm{MAA}\right)$ is normally performed in a separate safety procedure for estimation of the lung shunting fraction (LSF) and detection of potential extrahepatic distributions. Ideally, both this pre-treatment procedure and the treatment itself would be performed in a single setting, to minimize in-between changes in anatomy and catheter position [6]. Such a single-session procedure would require SPECT/CT in the intervention room for a smooth workflow, for which we are developing a compact mobile system [7-9], scanning to be performed in minutes, and reconstructions to be available within seconds.

This study compares the obtained image quality, reconstruction speed, and response to short scan time for the discussed fast neural network approach, a relatively slow but accurate Monte Carlobased reconstructor, and a commercially available clinical reconstructor, so that it becomes clear whether the neural network approach can provide reconstructions with quality similar to those obtained with Monte Carlo-based reconstruction, but now within seconds.

\section{Methods}

Convolutional neural networks are usually trained with pairs of low- and high-quality images. In nuclear medicine, however, the high-quality images (e.g. obtained with long scan time) can be substantially different from true distributions, due to technical limitations such as the limited spatial 
and energy resolution of the gamma camera. To prevent the neural network from learning the errors that arise from these limitations, this work introduced an additional intermediate reconstruction and projection step. The neural network could with this method potentially learn how to approach the true distribution better than the iterative image reconstructor. The separate steps are discussed in more detail below.

\section{Patient data}

Our retrospective study was approved by the local ethics committee, who also waived the need for informed consent of the patients involved. Projections of 128 SPECT/CT scans from the pretreatment ${ }^{99 \mathrm{~m} T \mathrm{Tc}-M A A}$ radioembolisation procedure were available. All scans were performed on a dual-head Symbia T16 detector system (Siemens Healthineers, Erlangen, Germany). Projections were obtained in 20 minutes under 120 angles using a low energy high resolution (LEHR) parallel hole collimator with a photopeak window between 129 and $150 \mathrm{keV}$ and a scatter window between 108 and $129 \mathrm{keV}$. Out of the 128 distributions, 100 were used for network training, 20 for validation, and 8 for testing purposes.

\section{Ground truth reconstruction}

The patient projections were first reconstructed using the Utrecht Monte Carlo System (UMCS) [10]. This software package has been validated for several isotopes [11-14] and is considered stateof-the-art. UMCS accounts for attenuation with the $\mu$-map obtained from the CT, resolution through point spread function modelling, and scatter using Monte Carlo simulation of the photon interactions in the body. A total of 10 iterations with 8 subsets was performed and no postreconstruction filter was applied. The volumes had $128 \times 128 \times 128$ voxels with $3.9 \mathrm{~mm}$ isotropic voxel size. The resulting reconstructions were set as ground truth distributions and were used for comparison of the reconstruction methods at a later stage.

\section{Synthetic volumes}

The performance of the neural network should improve when more unique volumes are available to train on. The 100 ground truth distributions in the training set were hence used to create additional synthetic volumes. From a random patient, the liver mask with corresponding attenuation map was first selected. A sphere with a random diameter of 7 to 20 pixels was then positioned at a random location in the liver and filled with a random patch of the activity distribution from another patient. The process was repeated until the entire liver mask was filled. The generated synthetic volumes were thus a composition of patches from tens of separate reconstructions. In total, 900 synthetic volumes were created with this method.

\section{Projection generation}

Projections of the 100 ground truth distributions and 900 synthetic volumes (with collimator and detector effects and up to ten orders of scatter) were generated using UMCS. The use of a high number of photon tracks combined with convolution-based forced detection yielded nearly noise-free projections. Poisson noise was then added so that the simulated projections became 


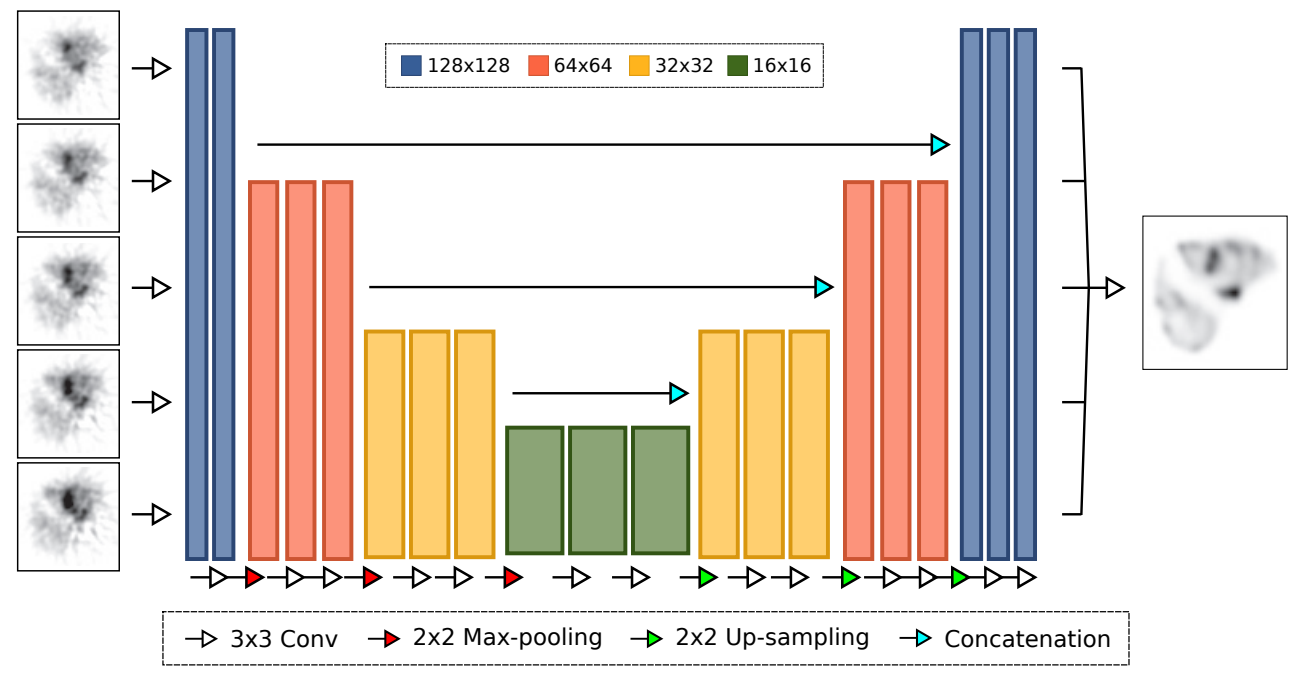

Figure 1: Schematic of the encoder-decoder convolutional neural network with five input slices as used in this study. Left are examples of the FBP input reconstructions and right the associated ground truth distribution.

representative of real detector measurements. Projections were simulated for two scan times: 20 $\mathrm{min}$, as is customary for a regular diagnostic SPECT/CT scan; and $5 \mathrm{~min}$, which we envision for use in interventional radioembolisation procedures. The total activity of the ground truth volumes was set to $150 \mathrm{MBq}$, as this is the average injected dose in the radioembolisation pre-treatment procedure in our hospital. The detector was configured with a single head, in anticipation of the compact mobile system mentioned in the introduction.

\section{Reconstruction methods}

Above projection sets were reconstructed using four different methods:

- Filtered back projection (FBP). The ramp filter was used in combination with Chang's correction [15] to compensate for attenuation (using the attenuation map from the CT scan). A post-reconstruction Gaussian filter of $5 \mathrm{~mm}$ full width at half maximum (FWHM) was applied to remove the most severe artefacts.

- Monte Carlo-based reconstruction (MC). The projections were reconstructed using the same reconstructor as used in the initial reconstruction (UMCS). A total of 10 iterations with 8 subsets was performed and no post-reconstruction filter was applied.

- Clinical reconstruction (CLINIC). An iterative reconstruction method, as can be found in state-of-the-art clinical methods (such as Flash3D in Siemens systems), was used. This reconstruction method included attenuation correction, resolution recovery, and used dualenergy window scatter correction [16]. Scattered photons were smoothed with a Gaussian filter of $5 \mathrm{~mm}$ FWHM and added to the reconstruction loop at fraction $k=0.5$. A post- 


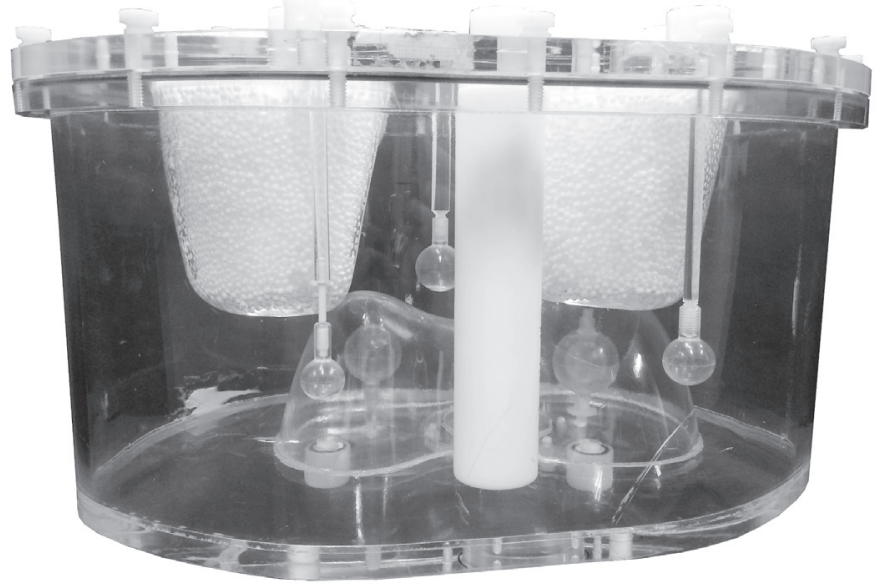

Figure 2: The anthropomorphic phantom with three extrahepatic volumes and one solid lesion and one lesion with a cold core inside the liver.

reconstruction Gaussian filter of $5 \mathrm{~mm}$ FWHM was employed and a total of 10 iterations with 8 subsets was performed. These settings were chosen as they are current clinical practice in our institute.

- Convolutional neural network approach (CNN). The projections were first reconstructed using FBP as above and then fed to the trained network to increase the image quality.

\section{Network design}

The neural network used a deep convolutional encoder-decoder structure (see Fig. 1), which is frequently used for denoising applications $[17,18]$. Network training was performed by minimizing the voxel-wise mean squared error of the FBP reconstructions with the combination of the 100 ground truth distributions and 900 synthetic volumes. The network consisted of layers with several resolutions, which were connected with each other via concatenation (to ensure small objects were not lost in training). Five adjacent slices per sample were used as input so that resolution in all directions was preserved. By inserting all 128 slices from FBP into the network, the entire volume was reconstructed. Separate networks were trained for both simulated scan times.

For the encoding layers, every step consisted of two $3 \times 3$ convolutional layers with ReLu activation function, followed by $2 \times 2$ max pooling. The decoding layers first upsampled the resolution and again used two $3 \times 3$ convolutional layers. Learning was performed using the ADAM optimizer [19] with a learning rate of $1 \mathrm{e}-4$. The data was fed to the network with a batch size of 128 . Training continued until no further decrease in the loss function was observed for 20 epochs. The training was performed using TensorFlow 1.7.0 [20] with Keras 2.1.6 [21]. 


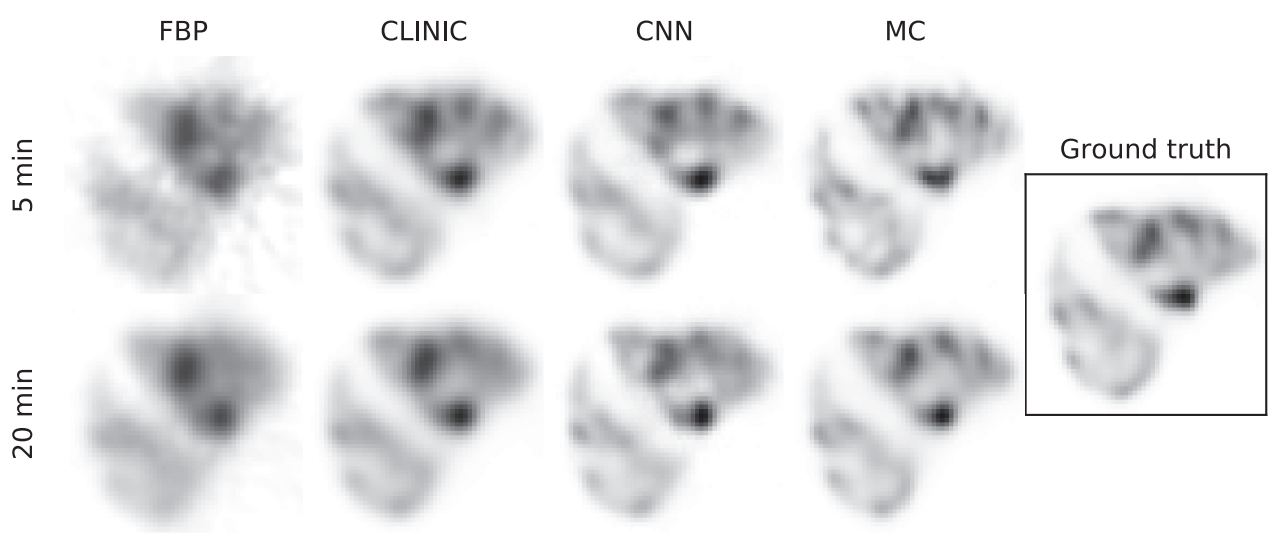

Figure 3: Reconstructed image slices of a representative patient distribution from the validation set, for the four reconstruction methods and two scan times. Additionally shown is the associated ground truth distribution.

\section{Network performance}

It was first studied whether the introduced reconstruction and projection step (by setting the initial reconstructed images as ground truth) performed better than when training directly to the Monte Carlo-based generated reconstructions. It was subsequently evaluated whether the augmentation of training data with synthetic volumes aided network performance by separately training with 0,300 , 600 , and 900 synthetic phantoms, in addition to the 100 ground truth distributions. The minimum acquired losses were used as a measure for network performance. Since the neural network is slightly sensitive to the random initial weights chosen, five realizations were performed per setting.

\section{Validation performance}

The mean squared error of the four reconstructions (normalized to the total reconstructed activity) with the associated ground truth was calculated for the two scan times and used as a quantitative measure for reconstruction quality for the 20 patients in the validation set. The difference of the LSF with the ground truth distributions was furthermore measured because this measure is often assessed in hepatic radioembolisation.

\section{Phantom measurements}

A phantom study was performed to evaluate to what extent the neural network approach could reconstruct true detector projections. An anthropomorphic phantom was adjusted from a commercially available phantom (Anthropomorphic Torso Phantom: ECT/TOR/P) by inclusion of three extrahepatic spheres (with volumes of 2.0,4.1, and $8.1 \mathrm{~mL}$ ) and one solid sphere $(15.7 \mathrm{~mL}$ ) and one sphere with cold core $(5.6 \mathrm{~mL}$ cold volume; $18.7 \mathrm{~mL}$ hot volume) inside the liver (see Fig. 2). The extrahepatic spheres were filled with 2.7 uptake ratio in relation to the liver background activity, for the spheres inside the liver, this ratio was 7.7. The lungs were filled with LSF of 5.2\%. The phantom was filled with water and had $157 \mathrm{MBq}$ total activity of ${ }^{99 \mathrm{~m}} \mathrm{Tc}$. The phantom was configured in this way to represent situations encountered in hepatic radioembolisation [22,23]. 


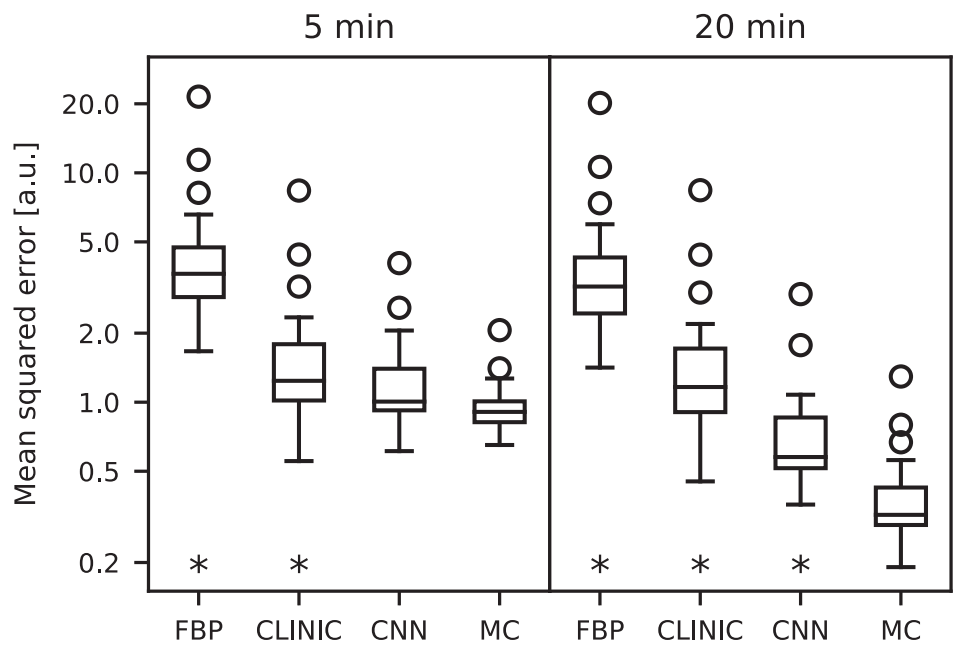

Figure 4: Mean squared error for the 20 distributions in the validation set, for the four reconstructions methods and the two scan times. The asterisk notes the reconstruction methods that were significantly different from the Monte Carlo-based reconstruction (Mann-Whitney $\mathrm{U}$ test at $p<0.01$ ).

The anthropomorphic phantom was scanned for $20 \mathrm{~min}$ on the same scanner with the same acquisition settings as in the patient scans but now with a single head. By uniform random subsampling from the obtained projections, projections with 5 min scan time were additionally acquired. Liver, sphere, and lung masks were generated from delineation on the low-dose CT. The uptake ratios of the solid spheres, contrast-to-noise ratio (CNR), and LSF were calculated from the reconstructions and compared with the values of the phantom. The CNR was calculated with the solid sphere inside the liver and the liver background.

\section{Original detector projections}

Reconstructions were finally performed of the 8 patient projections in the testing set to give an indication of the reconstruction performance on true detector projections for patient distributions with varying levels of activity. Since no ground truth is present for these cases, the reconstructions were solely visually compared.

\section{Results}

\section{Reconstruction time}

The CNN reconstruction was performed on the graphics processing unit (GPU), the other options used the central processing unit (CPU) with a single core, all on a Dell Precision $7810(2.60 \mathrm{GHz}$ Xeon E5-2640v3 and 64 GB RAM) with NVIDIA Quadro P6000. The time for reconstruction of an entire volume was approximately 2 sec using FBP and $5 \mathrm{sec}$ with CNN. The clinical reconstruction required $5 \mathrm{~min}$ and the Monte Carlo-based reconstruction required $19 \mathrm{~min}$. The CNN training took approximately 10 hours for the case of 900 synthetic volumes. 


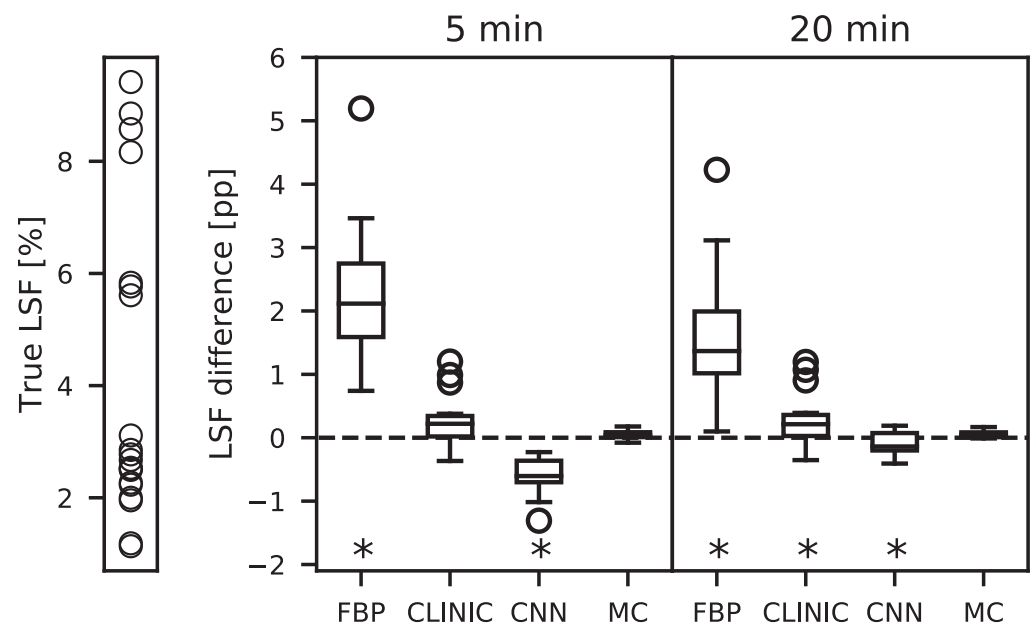

Figure 5: The difference in percent point (pp) of the LSF for the 20 distributions in the validation set, for the four reconstructions methods and the two scan times. Additionally shown are the LSFs found in the ground truth distribution. The asterisk notes the reconstruction methods that resulted in a significantly different LSF from the Monte Carlo-based reconstruction (Mann-Whitney U test at $p<0.01$ ).

\section{Network performance}

Directly training the FBP to the Monte Carlo-based reconstructions (with no synthetic volumes) resulted in a minimum validation loss of $1.31 \pm 0.02$, whereas with the introduced reconstruction and projection step it reduced to $1.22 \pm 0.01$. This indicated that the introduction of this step aided network performance.

The minimum validation loss was $1.22 \pm 0.01$ when no synthetic volumes were used and then decreased to $1.06 \pm 0.02$ for 300 volumes, $1.04 \pm 0.01$ for 600 volumes, and $1.01 \pm 0.01$ for 900 volumes. The introduction of synthetic volumes into the training set thus also improved network performance.

\section{Validation performance}

The reconstructions of one patient distribution from the validation set are shown in Fig. 3 for the four reconstruction methods and two scan times. For all reconstruction methods, it is evident that quality deteriorated with shorter scan time. FBP created reconstructions with severe artefacts and tumour contrast was low. Clinical reconstructions had no visible artefacts but had low contrast too. The CNN approach provided results that were comparable to the Monte Carlo-based reconstructions.

The mean squared error of the reconstructions with the associated ground truth distributions in the validation set is depicted in Fig. 4. These quantitative results showed that the neural network approach had the performance closest to the Monte Carlo-based reconstruction, for both scan times. 
Table 1: Lung shunting fraction, uptake ratio for the spheres, and contrast-to-noise ratio for the four reconstruction methods and two scan times, together with the values as configured in the phantom.

\begin{tabular}{ll|cccccc} 
& & LSF [\%] & Up. 2.0 mL & Up. 4.1 mL & Up. 8.1 mL & Up. 15.7 mL & CNR \\
\hline True values & & 5.2 & 2.7 & 2.7 & 2.7 & 7.7 & - \\
\hline $5 \mathrm{~min}$ & FBP & 6.4 & 0.5 & 0.8 & 1.0 & 4.1 & 6.5 \\
& CLINIC & 5.1 & 1.0 & 1.4 & 1.5 & 5.2 & 10.3 \\
& CNN & 4.7 & 1.7 & 2.3 & 2.0 & 6.6 & 12.1 \\
& MC & 5.8 & 2.0 & 2.3 & 2.2 & 6.5 & 11.0 \\
\hline \multirow{2}{*}{$20 \mathrm{~min}$} & FBP & 5.3 & 0.5 & 0.8 & 1.0 & 4.1 & 6.6 \\
& CLINIC & 4.7 & 1.0 & 1.4 & 1.5 & 5.1 & 10.5 \\
& CNN & 5.1 & 1.9 & 2.3 & 2.3 & 7.2 & 12.5 \\
& MC & 5.2 & 2.0 & 2.2 & 2.2 & 6.5 & 11.6
\end{tabular}

The difference of the LSF obtained with the four reconstruction methods with that from the ground truth distributions is shown in Fig. 5. The Monte Carlo-based reconstruction and clinical reconstruction provided the lowest difference. FBP resulted in an overestimation of several percent point ( $\mathrm{pp}$ ) and the $\mathrm{CNN}$ reconstruction gave a slight underestimation for the $5 \mathrm{~min}$ scan. The values from clinical, CNN, and Monte Carlo-based reconstruction were all, however, within $2 \mathrm{pp}$ difference.

\section{Phantom measurements}

The quantitative measures from the reconstructions of the phantom experiment are compiled in Tab. 1, together with the true values as configured in the phantom. The uptake ratios became relatively worse for spheres of decreasing size, due to the partial volume effect. The Monte Carlobased and $\mathrm{CNN}$ reconstructions provided similar ratios, whereas the found values were lower for clinical reconstruction and FBP. The CNN, clinical, and $\mathrm{MC}$ reconstructions gave comparable LSF values; a higher fraction was obtained with FBP. The CNR provided values that were in the same range for both clinical, CNN, and Monte Carlo-based reconstruction; again, only FBP had substantially lower values.

\section{Original detector projections}

Five reconstructions obtained from the real detector projections of the 8 patients in the testing set are visualized in Fig. 6. These projection sets were generally obtained a few hours after injection (in contrast to our envisioned interventional scanning) and thus did not carry the $150 \mathrm{MBq}$ as in the simulations. The network from the $20 \mathrm{~min}$ scan time was used for the CNN because this network was trained with the closest activity levels. No ground truth is available for these images. Visual inspection, however, showed that $\mathrm{CNN}$ and Monte Carlo-based reconstruction had the same features present. This indicated that the neural network approach was also able to generalize well to true detector measurements for patients. 


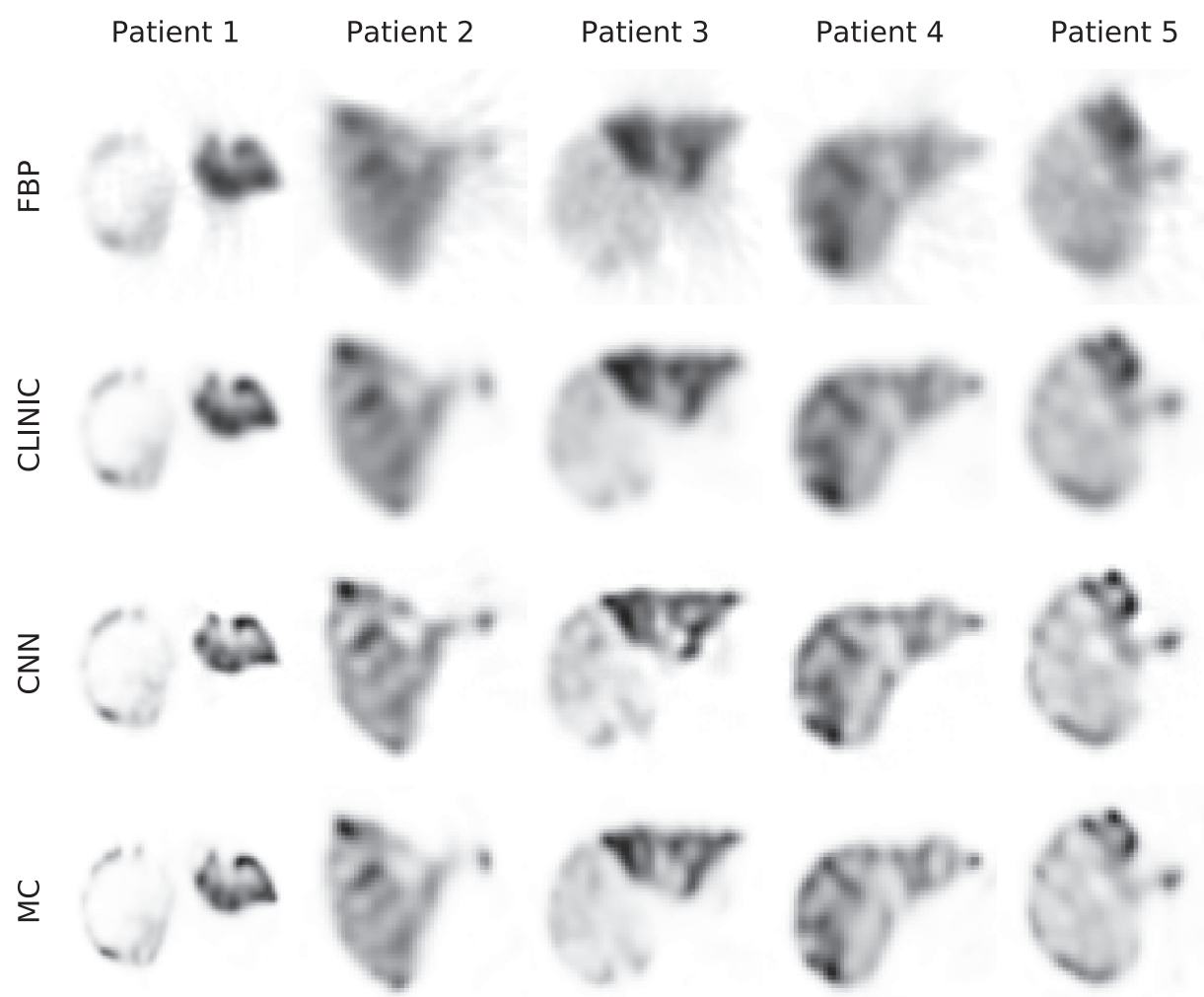

Figure 6: Reconstruction slices of five representative distributions from the test set, for the four reconstruction methods.

\section{Discussion}

This study showed that FBP with an image enhancement convolutional neural network in SPECT/ CT can perform reconstruction within seconds. From the evaluation of the validation set, the phantom experiment, and visual inspection of the testing set, it was found that image quality close to that of the Monte Carlo-based reconstructor was retrieved. The neural network approach additionally performed well for short scans, which would be required for use in interventional SPECT/CT scanning.

This study is one of the first to illustrate the use of deep learning in SPECT. In other modalities, such as positron emission tomography (PET) [24,25], the use of an image enhancement neural network has previously been explored. The mentioned studies, however, had the primary aim of limiting the injected dose and made no use of the acceleration in reconstruction time as is illustrated in this study. Also, the method of creating synthetic volumes to augment the training data has not shown before in nuclear medicine. 
Although this study focused on potential implementation in the pre-treatment procedure of radioembolisation, we believe that our approach is applicable to all forms of SPECT/CT. We envision that one use would be to quickly validate whether a scan was made without any motion artefacts. The network in this work was only trained with distributions from radioembolisation. It is unknown how well the network generalizes to other distributions. It might prove that the neural network needs to be trained for each procedure separately to ensure optimal results.

In the current clinical practice of radioembolisation, the pre-treatment procedure of radioembolisation has two major aims: determination of the lung shunting fraction and detection of potential extrahepatic distributions. Both measures were accurately retrieved from the reconstructions from the neural network approach in the phantom experiment. For the patient distributions, it was found that the difference of the LSF obtained with CNN and the ground truth was within 2 percent point, which can be considered clinically sufficient.

A limitation of this study is that it was not shown that extrahepatic depositions were also accurately detected in the patient distributions. Because these depositions are not commonly found in our institute and we did not perform a selection on which distributions to include in training or validation, there were not enough cases to draw conclusions on. However, by means of the phantom experiment and by illustrating the visual quality of the images obtained with CNN, we are confident that patient extrahepatic depositions would also be accurately detected.

It was shown that upon increasing the number of synthetic volumes, the neural network performance increased. A further increase in quality might be achievable when including more volumes or adding more layers to the network. However, this would simultaneously increase the required GPU memory. Such computational aspects are currently holding back further increase in network performance. With a smarter selection of distributions (i.e. assembling the set with the highest diversity), it might be possible to achieve improved results at the same computational cost.

The clinical and Monte Carlo-based reconstructions were performed using a single core in this study. Substantial acceleration can be achieved when the code is parallelized and a dedicated workstation with many CPU cores is used, which might bring the Monte Carlo-based reconstruction time to a clinically acceptable value. The use of GPUs is also very promising for shortening the reconstruction time [26]. An advantage of the neural network approach is that the reconstruction time is almost independent of the matrix size and the number of iterations.

A potential application for the developed method was proposed for interventional scanning in radioembolisation. This would also require short scan times and a scanner in the intervention room to ensure a smooth workflow. As for the short scan times, it was recently shown that one can move toward substantially reduced scan times $(<10 \mathrm{~min})$ and still obtain accurate results on various quantitative measures [27]. As for the scanner, a mobile compact system that can be included in the intervention room [7-9] is currently being built and it is expected that this system is available for patient studies soon. Combined with the fast reconstruction in this work, we believe that all elements are present to enable interventional SPECT imaging in clinical practice. 


\section{Conclusions}

FBP with an image enhancement convolutional neural network can provide SPECT reconstructions similar in quality to those obtained with Monte Carlo-based reconstruction for the pre-treatment procedure of hepatic radioembolisation within seconds.

\section{References}

1. Cherry SR, Sorenson JA, Phelps ME. Tomographic Reconstruction in Nuclear Medicine. In: Physics in Nuclear Medicine. $4^{\text {th }}$ ed. Saunders, 270-271 (2012)

2. Gustafsson J, Brolin G, Ljungberg M. Monte Carlo-based SPECT reconstruction within the SIMIND framework. Phys. Med. Biol. 63(24):245012 (2018)

3. Dewaraja YK, Chun SY, Srinivasa RN, et al. Improved quantitative ${ }^{90} \mathrm{Y}$ bremsstrahlung SPECT/CT reconstruction with Monte Carlo scatter modeling. Med. Phys. 44(12):6364-6376 (2017)

4. Litjens G, Kooi T, Bejnordi BE, et al. A survey on deep learning in medical image analysis. Med Image Anal. 42:60-88 (2017)

5. Kennedy A, Coldwell D, Sangro B, Wasan H, Salem R. Radioembolization for the treatment of liver tumors: General principles. Am J Clin Oncol Cancer Clin Trials. 35(1):91-99 (2012)

6. Gabr A, Kallini JR, Gates VL, et al. Same-day ${ }^{90} \mathrm{Y}$ radioembolization: implementing a new treatment paradigm. Eur J Nucl Med Mol Imaging. 43(13):2353-2359 (2016)

7. Van der Velden S, Kunnen B, Koppert WJC, et al. A Dual-layer Detector for Simultaneous Fluoroscopic and Nuclear Imaging, Radiology 290(3):833-838 (2019)

8. Dietze MMA, Kunnen B, Van der Velden S, Steenbergen JHL, Koppert WJC, Viergever MA, De Jong HWAM. Performance of a Dual-Layer Scanner for Hybrid SPECT/CBCT, Phys. Med. Biol. 64(10):105020 (2019)

9. Dietze MMA, Bastiaannet R, Kunnen B, Van der Velden S, Lam MGEH, Viergever MA, De Jong HWAM. Respiratory Motion Compensation in Interventional Liver SPECT using Simultaneous Fluoroscopic and Nuclear Imaging, Med. Phys. 46(8):3496-3507 (2019)

10. De Jong HWAM, Slijpen ETP, Beekman FJ. Acceleration of Monte Carlo SPECT simulation using convolution-based forced detection. IEEE Trans Nucl Sci. 48(1):58-64 (2001)

11. Dietze MMA, Van der Velden S, Lam MGEH, Viergever MA, De Jong HWAM, Fast quantitative reconstruction with focusing collimators for liver SPECT. EJNMMI Phys 5(1):28 (2018)

12. Xiao J, De Wit TC, Staelens SG, Beekman FJ. Evaluation of 3D Monte Carlo-based scatter correction for ${ }^{99 \mathrm{~m}}$ Tc cardiac perfusion SPECT. J Nucl Med. 47(10):1662-1669 (2006)

13. Elschot M, Lam MGEH, Van den Bosch MAAJ, Viergever MA, De Jong HWAM. Quantitative Monte Carlo-based ${ }^{90}$ Y SPECT reconstruction. J Nucl Med. 54(9):1557-1563 (2013)

14. Elschot M, Smits MLJ, Nijsen JFW, et al. Quantitative Monte Carlo-based holmium-166 SPECT reconstruction. Med Phys. 40(11):112502 (2013)

15. Chang LT. A method for attenuation correction in radionuclide computed tomography. IEEE Trans Nucl Sci. 25(1):638-643 (1978)

16. Jaszczak RJ, Greer KL, Floyd CE, Harris CC, Coleman RE. Improved SPECT Quantification Using Compensation for Scattered Photons. J Nucl Med. 25(8):893-900 (1984)

17. McCann MT, Jin KH, Unser M. Convolutional neural networks for inverse problems in imaging: A review. IEEE Signal Process. Mag. 201:85-95 (2017) 
18. Ronneberger O, Fischer P, Brox T. U-net: Convolutional networks for biomedical image segmentation. MICCAI:234-241 (2015)

19. Kingma DP, Ba JL. ADAM: A Method for Stochastic Optimization. ICLR (2015)

20. Abadi M, Barham P, Chen J, et al. TensorFlow: A System for Large-Scale Machine Learning. Oper Syst Des Implement 16:265-284 (2016)

21. Chollet, F. Keras. https://github.com/fchollet/keras (2015)

22. Prince JF, Van Rooij R, Bol GH, De Jong HWAM, Van den Bosch MAAJ, Lam MGEH. Safety of a Scout Dose Preceding Hepatic Radioembolization with ${ }^{166}$ Ho Microspheres. J Nucl Med. 56(6):817-823 (2015)

23. Van den Hoven AF, Rosenbaum CENM, Elias SG, De Jong HWAM, Koopman M, Verkooijen HM, Alavi A, Van den Bosch MAAJ, Lam MGEH. Insights into the Dose-Response Relationship of Radioembolization with Resin ${ }^{90}$ Y-Microspheres: A Prospective Cohort Study in Patients with Colorectal Cancer Liver Metastases. J Nucl Med. 57(7):1014-1019 (2016)

24. Xu J, Gong E, Pauly J, Zaharchuk G. 200x Low-dose PET Reconstruction using Deep Learning. http:// arxiv.org/abs/1712.04119 (2017)

25. Jiao J, Ourselin S. Fast PET reconstruction using Multi-scale Fully Convolutional Neural Networks. http://arxiv.org/abs/1704.07244 (2017)

26. Rydén T, Heydorn Lagerlöf J, Hemmingsson J, et al. Fast GPU-based Monte Carlo code for SPECT/CT reconstructions generates improved 177Lu images. EJNMMI Phys. 5(1) (2018)

27. Van der Velden S, Dietze MMA, Viergever MA, De Jong HWAM. Fast technetium-99m liver SPECT for evaluation of the pretreatment procedure for radioembolization dosimetry. Med Phys. 46(1):345-355 (2019) 



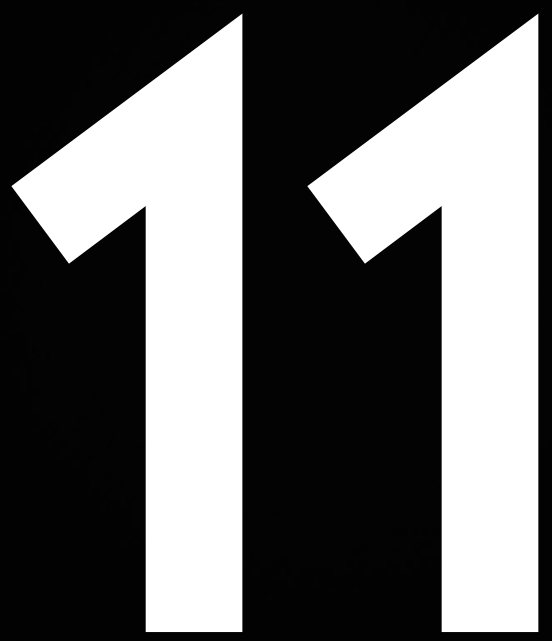




\section{SUMMARY AND DISCUSSION}




\section{Summary}

This dissertation has introduced several methodological contributions that are required to enable nuclear image guidance in the interventional suite. The contributions have primarily been evaluated for use in radioembolization since this treatment is one of the most promising candidates to benefit from interventional nuclear imaging.

\section{Interventional Scanner}

The first task to enable interventional nuclear imaging is to develop a compact and flexible scanner with an open gantry that can also provide an anatomical reference for the nuclear images. A design was proposed and evaluated with a prototype scanner in CHAPTER 2 and with a full-scale device in CHAPTER 3. The reconstruction quality of the interventional scanner was lower than that of a conventional clinical scanner due to the system design. However, it was shown that metrics relevant for radioembolization were accurately retrieved from the images.

Instead of developing a new scanner, it might also be possible to adjust the scanner that is already available in the intervention room. In CHAPTER 4, it was demonstrated that the x-ray flat panel detector of the CBCT scanner can be used for nuclear imaging. While the sensitivity of a flat panel detector is lower than that of a conventional gamma camera, the intrinsic spatial resolution is much better. With this property, the reconstruction quality of a conventional gamma camera was approached.

\section{Motion Compensation}

The nuclear reconstruction should be accurate if the distribution is used for the planning of radionuclide therapies. Patient respiratory motion is a major degrading factor for the reconstruction accuracy and should hence be compensated. Conventional methods to compensate for respiratory motion require external apparatus or high activity levels. Both are undesired in an interventional setting. A new method to compensate for respiratory motion was proposed by making use of the simultaneous acquisition of nuclear and fluoroscopic images in the interventional scanner. The method was evaluated with simulations in CHAPTER 5 and with a phantom study in CHAPTER 6. The motion compensation method substantially improved the image quality of the nuclear reconstructions in both studies.

\section{Faster Scanning}

The interventional scan duration should be as short as possible in order not to considerably prolong the time spent in the intervention room. Converging collimators (e.g. the cone beam collimator and the multifocal collimator) can focus on a specific organ to achieve higher sensitivity and hence could be used to shorten the scan duration. Quantitative reconstruction software for such collimators was developed and evaluated with digital simulations in CHAPTER 7. The quantitative reconstructor of the multifocal collimator was validated with phantom experiments in CHAPTER 8. The focusing collimators substantially reduced the scan duration for both studies. 
A different method of shortening the interventional scan duration, by evaluating the radionuclide distribution during scanning, was proposed in CHAPTER 9. Multiple fast scans of one minute each were made and the reconstruction was updated with the newly obtained counts after every rotation. The scan was hence terminated when the acquired images were sufficiently stable. Digital simulations, phantom experiments, and a retrospective patient study demonstrated that the scan duration could be substantially reduced without loss of diagnostic information.

\section{Faster Reconstruction}

The nuclear reconstructions should be available quickly after scanning to ensure that the valuable time in the intervention room does not go to waste. Current state-of-the-art reconstructors require several minutes to complete. In CHAPTER 10, a different method for reconstruction was explored. This approach first reconstructed the projections using filtered back projection and then passed the results to an image enhancement convolutional neural network. The deep learning reconstruction method provided images with a quality similar to that of state-of-the-art reconstruction but completed within seconds. 


\section{Discussion}

This dissertation has introduced several methodological contributions to enable interventional nuclear imaging. The majority of the presented techniques can be combined in a single system. Such a system would be able to create motion-compensated reconstructions in the intervention room within minutes of scanning and seconds of reconstruction, and would hence meet the requirements for use in clinical practice. The developments have been primarily investigated for radioembolization as a potential application for interventional nuclear imaging.

\section{Clinical Introduction}

A first clinical study is planned to investigate the potential of the interventional scanner to aid the radioembolization workflow. The primary objective of this study is feasibility and safety: it will be evaluated whether interventional nuclear imaging can be smoothly incorporated into the procedure workflow and whether any adverse side-effects occur when applying interventional nuclear imaging. The secondary objectives include the study of potential dynamic uptake behavior of ${ }^{99}{ }^{\mathrm{m}} \mathrm{Tc}-\mathrm{MAA}$, the comparison between the dosimetric quality of the interventional reconstruction with that of a conventional reconstruction, and the potential benefit of motion compensation for the nuclear image interpretation. These secondary objectives will be evaluated on a case-by-case basis since the outcome of these tasks is expected to vary across patients.

\section{Radioembolization Workflow}

The merging of the scout dose procedure (i.e. the simulation of the treatment procedure which can be used for dosimetry-based activity planning) with the treatment in a single-session procedure will provide radionuclide distributions that contain a mixture of ${ }^{99 \mathrm{~m}} \mathrm{Tc}-\mathrm{MAA}$ particles with ${ }^{90} \mathrm{Y} /{ }^{166} \mathrm{Ho}$ microspheres. This mixture causes a more complicated energy spectrum since the scattered photons from one radionuclide will influence measurements in the energy window of the other. However, when this energy spectrum is precisely incorporated in the software, accurate reconstructions can be achieved [1].

A prerequisite for accurate dosimetry in radioembolization is that the particles used in the pretreatment procedure should be representative of the microspheres. Concerns have been raised on the predictive accuracy of the ${ }^{99 \mathrm{~m}} \mathrm{Tc}-\mathrm{MAA}$ particles due to their aberrant size [2,3]. Promising research is ongoing on the use of small amounts (i.e. scout dose) of ${ }^{90} \mathrm{Y}[4,5]$ or ${ }^{166} \mathrm{Ho}[6-8]$ microspheres in the pre-treatment procedure (equivalent to the particles used for treatment). Scout doses may also be imaged with the interventional SPECT scanner. For imaging of these particles, the scanner should be mounted with a high-energy collimator (instead of a low-energy one in case of ${ }^{99 \mathrm{~m}} \mathrm{Tc}$ ), because of the higher photon energies involved [9].

\section{Interventional Scanner}

In the dual-layer detector design of the interventional scanner, the flat panel detector was positioned in front of the gamma camera to enable the simultaneous acquisition of intrinsically registered nuclear and fluoroscopic projections. This choice resulted in a lower SPECT reconstruction 
quality (due to increased gamma attenuation and deteriorated spatial resolution), lower CBCT reconstruction quality (due to the removal of the anti-scatter grid), a non-uniform response in the nuclear projections (caused by components in the flat panel detector), and gamma camera performance deterioration at higher beam strengths (due to crystal afterglow and photomultipliertube saturation). The planned clinical study will investigate whether obtaining such simultaneous intrinsically registered nuclear and fluoroscopic information has a clinical benefit. If this is not the case, a simpler design (i.e. with the flat panel detector positioned elsewhere on the gantry) may be considered. Such a design will more closely resemble a conventional SPECT/CT scanner, which may be more difficult to integrate into the intervention room.

The gamma camera in the dual-layer detector design suffers from severe artifacts at higher x-ray beam strengths [10]. There exist materials for the photon detection that are more resilient to this effect (for instance, $\mathrm{CeBr}_{3}$ and $\mathrm{CdZnTe}$ ) but these detectors have high associated production costs [11]. An alternative (cost-effective) solution was proposed by using an off-focus geometry. This approach increased the tolerable beam strength but was a sub-optimal solution since the design introduced a mismatch in the registration of the fluoroscopic and nuclear projections. Even with off-focus design, it was not possible to perform simultaneous nuclear and fluoroscopic imaging with the beam strengths that are currently clinically used $(120 \mathrm{kVp})$.

It was demonstrated that the interventional scanner provides a worse reconstruction quality than a clinical scanner due to its lower sensitivity and poorer resolution. These characteristics will limit the scanner usage for small or low-activity radionuclide accumulations. It is unknown to what extent this will limit the applicability of the interventional scanner for image guidance purposes. Most likely, it will be sufficient to accurately image relatively large volumes (e.g. liver compartments in radioembolization used for dosimetry purposes [12]).

The quality obtained from the interventional nuclear scanner may be improved by relocating the detector electronics of the flat panel detector. This modification would reduce the thickness of the flat panel detector, which in turn would improve the spatial resolution and the sensitivity of the gamma camera.

\section{Motion Compensation}

The simulation study on respiratory motion compensation with the interventional scanner investigated the potential of extracting a motion model (i.e. a map of the tissue deformations over time) from the gated CBCT reconstructions and incorporating this information into the SPECT reconstruction. The experimental study with the interventional scanner investigated a different method by extracting only the motion signal (i.e. the state of the respiratory motion; inhalation or exhalation) and using this information to gate the nuclear counts into motion bins. Both approaches substantially increased the quantitative accuracy of the SPECT reconstructions. The motion model technique resulted in lower noise in the SPECT reconstruction than gating, but the performance was unstable at lower dose levels and several assumptions were required. For this reason, the gating approach is the preferred motion compensation method for the planned clinical study. 


\section{Interventional Imaging}

There are already several other devices that are specifically developed for use in the intervention room. For SPECT, hand-held cameras or compact and mobile gamma cameras are sometimes employed $[13,14]$. Although these devices provide much lower image quality than diagnostic SPECT/CT and lack quantitative feasibility, they have the advantage that they are easy to work with and are valued for their smooth integration into the intervention room. However, their field of view is small and no real-time anatomical reference is provided, which complicates the interpretation of the nuclear images.

For PET [15,16], MRI [17,18], and CT [19,20], interventional scanner designs have been introduced as well. These designs function by moving the scanner back-and-forth to the operating table (or vice-versa) when the patient needs to be imaged. An important disadvantage of these designs is that the intervention room needs to be specifically built around the scanner, which is costly and space-consuming. These are practical considerations that could limit the widespread application of interventional imaging.

Still, there are several ways in which the above-mentioned interventional scanners may aid the radioembolization workflow. The interventional CT scanner may be used to acquire contrastenhanced images that accurately visualize the contrast-enhanced vasculature in the targeted tissue, which can then be used for treatment planning [21]. A disadvantage of this method is that the physical properties of the injected particles are not taken into account (i.e. it is not known where the particles lodge), complicating more sophisticated dosimetry methods.

Both interventional CT and interventional MRI can be used to visualize the distribution of dense/ paramagnetic microspheres $\left({ }^{166} \mathrm{Ho}\right)$. A disadvantage of both techniques is the limited sensitivity for small amounts of microspheres. In addition, for interventional MRI, the intervention room is a very hostile environment because of the magnetic objects that are conventionally used during the intervention. Many changes to the interventional workflow are hence required to enable interventional MRI, which could be a substantial hurdle for its implementation.

The interventional PET scanner may be used to visualize ${ }^{90} \mathrm{Y}$ microspheres since this radionuclide also emits positrons (albeit at a very small branching fraction). Such PET imaging provides accurate images when therapeutic activity levels are concerned [22]. However, for imaging activity levels that may be used for planning purposes (i.e. scout dose), the PET image is likely not sufficient for accurate dosimetry $[4,5]$. The dosimetric quality obtained with SPECT remained accurate at diagnostic activity levels.

The interventional scanner design in this dissertation was constructed to enable dynamic imaging with a real-time anatomical reference. However, if one is only interested in obtaining nuclear images, an interventional design similar to the above configurations (with the scanner translating back-andforth to the operation table) could be constructed. Such a scanner would be comparable to the conventional clinical scanners and can be expected to provide a similar reconstruction quality. 


\section{Future Perspectives}

Radionuclides are increasingly being used in patient treatments. These innovations require technical support in the form of imaging equipment and data analysis. This dissertation has introduced the methodological contributions required to enable interventional nuclear image guidance and evaluated these methods retrospectively on a case-by-case basis. A substantial effort is now required to combine all of the methods into a single robust imaging pipeline. Additional features can be added when this task is completed. It may e.g. be beneficial to overlay tumor delineations (previously made on e.g. a high-quality CT or MRI) on the radionuclide distribution obtained in the intervention room to quantitatively determine their obtained radiation doses.

The developments in nuclear image guidance will likely place higher demands on the associated data analysis and equipment in the intervention room. When it becomes possible to precisely monitor tumor doses, it may e.g. become possible to incorporate knowledge on tumor dose-response relationships that have already been extensively studied in radiotherapy. In turn, it should become possible to precisely regulate the amount of activity that is injected in the patient, which e.g. requires developments in the radionuclide injection mechanisms. All of these technological innovations have to be precisely monitored on their performance. This requires personnel that has sufficient expertise in these areas. Nuclear medicine clinical physicists will likely play an increasingly important role in the coming years for the optimization of interventional procedures involving radionuclides. The performance of nuclear medicine will be only as good as the technological support it receives.

An interventional nuclear scanner has now been constructed that can be easily incorporated into existing clinical workflows. This allows to study its performance in many interventional procedures involving radionuclides. These studies will determine which elements of the scanner are most important (e.g. how good the planar nuclear images should be in quality, whether the images should be quantitative) so that the scanner design can be optimized and potentially produced in larger numbers. This could allow other institutes to similarly investigate whether interventional nuclear imaging is beneficial in their innovative procedures involving radionuclides and may open up a new area of research. 


\section{References}

1. van Rooij R, Braat AJ, de Jong HW, Lam MG. Simultaneous ${ }^{166} \mathrm{Ho} /{ }^{99 m} \mathrm{Tc}$ dual-isotope SPECT with Monte Carlo-based downscatter correction for automatic liver dosimetry in radioembolization. EJNMMI physics. 2020;7(13).

2. Wondergem M, Smits ML, Elschot M, de Jong HW, Verkooijen HM, van den Bosch MA, Nijsen JF, Lam MG. ${ }^{99 m}$ Tc-macroaggregated albumin poorly predicts the intrahepatic distribution of ${ }^{90} \mathrm{Y}$ resin microspheres in hepatic radioembolization. Journal of Nuclear Medicine. 2013;54(8):1294-301.

3. Elschot M, Nijsen JF, Lam MG, Smits ML, Prince JF, Viergever MA, van den Bosch MA, Zonnenberg BA, de Jong HW. 99m Tc-MAA overestimates the absorbed dose to the lungs in radioembolization: a quantitative evaluation in patients treated with 166 Ho-microspheres. European journal of nuclear medicine and molecular imaging. 2014;41(10):1965-75.

4. Kunnen B, Dietze MM, Braat AJ, Lam MG, Viergever MA, de Jong HW. Feasibility of imaging ${ }^{90} \mathrm{Y}$ microspheres at diagnostic activity levels for hepatic radioembolization treatment planning. Medical Physics. 2020;47(3):1105-14.

5. Kunnen B, van der Velden S, Bastiaannet R, Lam MG, Viergever MA, de Jong HW. Radioembolization lung shunt estimation based on a ${ }^{90} \mathrm{Y}$ pretreatment procedure: A phantom study. Medical Physics. 2018;45(10):4744-53.

6. Braat AJ, Prince JF, van Rooij R, Bruijnen RC, van den Bosch MA, Lam MG. Safety analysis of holmium-166 microsphere scout dose imaging during radioembolisation work-up: a cohort study. European radiology. 2018;28(3):920-8.

7. Prince JF, van Rooij R, Bol GH, de Jong HW, van den Bosch MA, Lam MG. Safety of a scout dose preceding hepatic radioembolization with ${ }^{166} \mathrm{Ho}$ microspheres. Journal of Nuclear Medicine. 2015;56(6):817-23.

8. Smits ML, Dassen MG, Prince JF, Braat AJ, Beijst C, Bruijnen RC, de Jong HW, Lam MG. The superior predictive value of ${ }^{166} \mathrm{Ho}$-scout compared with ${ }^{99 \mathrm{~m}} \mathrm{Tc}$-macroaggregated albumin prior to ${ }^{166} \mathrm{Ho}$ microspheres radioembolization in patients with liver metastases. European journal of nuclear medicine and molecular imaging. 2019:47:798-806.

9. Elschot M, Nijsen JF, Dam AJ, de Jong HW. Quantitative evaluation of scintillation camera imaging characteristics of isotopes used in liver radioembolization. PLoS One. 2011;6(11).

10. Koppert WJ, van der Velden S, Steenbergen JH, de Jong HW. Impact of intense x-ray pulses on a NaI (Tl)-based gamma camera. Physics in medicine and biology. 2018;63(6):065006.

11. Koppert WJ, Dietze MM, van der Velden S, Steenbergen JL, de Jong HW. A comparative study of $\mathrm{NaI}(\mathrm{Tl}), \mathrm{CeBr}_{3}$, and $\mathrm{CZT}$ for use in a real-time simultaneous nuclear and fluoroscopic dual-layer detector. Physics in Medicine \& Biology. 2019;64(13):135012.

12. Bastiaannet R, Kappadath SC, Kunnen B, Braat AJ, Lam MG, de Jong HW. The physics of radioembolization. EJNMMI physics. 2018;5(1):22.

13. Tsuchimochi M, Hayama K. Intraoperative gamma cameras for radioguided surgery: technical characteristics, performance parameters, and clinical applications. Physica Medica. 2013;29(2):126-38.

14. Siman W, Cheenu Kappadath S. Performance characteristics of a new pixelated portable gamma camera. Medical physics. 2012;39(6):3435-44.

15. Tempany CM, Jayender J, Kapur T, Bueno R, Golby A, Agar N, Jolesz FA. Multimodal imaging for improved diagnosis and treatment of cancers. Cancer. 2015;121(6):817-27.

16. Shyn PB. Interventional positron emission tomography/computed tomography: state-of-the-art. Techniques in vascular and interventional radiology. 2013;16(3):182-90. 
17. White MJ, Thornton JS, Hawkes DJ, Hill DL, Kitchen N, Mancini L, McEvoy AW, Razavi R, Wilson S, Yousry T, Keevil SF. Design, Operation, and Safety of Single-Room Interventional MRI Suites: Practical Experience From Two Centers. Journal of magnetic resonance imaging.;41(1):34-43.

18. Black PM, Moriarty T, Alexander E, Stieg P, Woodard EJ, Gleason PL, Martin CH, Kikinis R, Schwartz RB, Jolesz FA. Development and implementation of intraoperative magnetic resonance imaging and its neurosurgical applications. Neurosurgery. 1997;41(4):831-45.

19. Lunsford LD, Parrish R, Albright L. Intraoperative imaging with a therapeutic computed tomographic scanner. Neurosurgery. 1984;15(4):559-61.

20. Uhl E, Zausinger S, Morhard D, Heigl T, Scheder B, Rachinger W, Schichor C, Tonn JC. Intraoperative computed tomography with integrated navigation system in a multidisciplinary operating suite. Operative Neurosurgery. 2009;64:231-9.

21. Roncali E, Taebi A, Foster C, Vu CT. Personalized Dosimetry for Liver Cancer Y-90 Radioembolization Using Computational Fluid Dynamics and Monte Carlo Simulation. Annals of Biomedical Engineering. 2020;48:1499-1510.

22. Kunnen B, Beijst C, Lam MG, Viergever MA, de Jong HW. Comparison of the Biograph Vision and Biograph mCT for quantitative ${ }^{90} \mathrm{Y}$ PET/CT imaging for radioembolisation. EJNMMI physics. $2020 ; 7(1): 1-3$. 


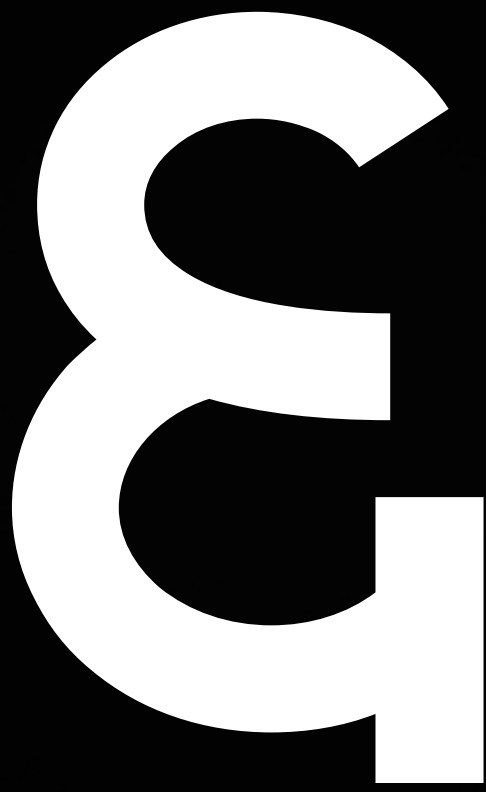


ADDENDA 


\section{Nederlandse Samenvatting}

Dit proefschrift heeft verschillende methodologische bijdragen geïntroduceerd die nodig zijn om interventionele nucleaire beeldvorming mogelijk te maken. De bijdragen zijn primair geëvalueerd voor het gebruik bij radio-embolisatie, omdat deze behandeling een van de meest veelbelovende kandidaten is om te profiteren van interventionele beeldvorming.

\section{Interventionele Scanner}

De eerste taak om interventionele nucleaire beeldvorming mogelijk te maken, is het ontwikkelen van een compacte en flexibele scanner die voldoende open ruimte heeft en ook een anatomische referentie voor de nucleaire beelden kan bieden. Een ontwerp werd voorgesteld en geëvalueerd met een prototype in HOOFDSTUK 2 en met een volledig apparaat in HOOFDSTUK 3. De reconstructiekwaliteit van de ontwikkelde scanner was lager dan dat van een conventionele klinische scanner vanwege het systeemontwerp. Er werd echter aangetoond dat de metingen die relevant zijn voor radio-embolisatie nauwkeurig konden worden bepaald in de beelden.

In plaats van het ontwikkelen van een nieuwe scanner, is het misschien ook mogelijk om de scanner aan te passen die al beschikbaar is in de interventieruimte. In HOOFDSTUK 4 werd aangetoond dat de detector van de CBCT-scanner ook kan worden gebruikt voor nucleaire beeldvorming. Hoewel de gevoeligheid van deze detector lager is dan die van een conventionele gammacamera, is de intrinsieke spatiële resolutie veel beter. Met deze eigenschap kon de reconstructiekwaliteit van een conventionele gamma-camera worden benaderd.

\section{Bewegingscompensatie}

De nucleaire reconstructie moet nauwkeurig zijn als de verdeling wordt gebruikt voor het plannen van radionuclide therapieën. De ademhalingsbeweging van een patiënt is een belangrijke verslechterende factor voor de nauwkeurigheid van de reconstructie en moet daarom worden gecompenseerd. Conventionele methoden om ademhalingsbewegingen te compenseren vereisen externe apparaten of veel geïnjecteerde activiteit. Beide zijn ongewenst in een interventionele omgeving. Daarom werd een nieuwe methode ontwikkeld om de ademhalingsbeweging te compenseren die gebruikmaakt van de gelijktijdige acquisitie van nucleaire en fluoroscopische beelden met de interventionele scanner. De methode werd geëvalueerd met simulaties in HOOFDSTUK 5 en met een fantoomstudie in HOOFDSTUK 6. De methode voor bewegingscompensatie verbeterde de beeldkwaliteit van de nucleaire reconstructies in beide onderzoeken aanzienlijk.

\section{Sneller Scannen}

De duur van de nucleaire scan moet zo kort mogelijk zijn om de tijd die in de interventieruimte wordt doorgebracht niet aanzienlijk te verlengen. Convergerende collimatoren (bijv. de kegelbundelcollimator en de multifocale collimator) kunnen op een specifiek orgaan worden gericht om een hogere gevoeligheid te bereiken en kunnen daarom worden gebruikt om de scanduur te verkorten. Kwantitatieve reconstructie-algoritmes werden ontwikkeld voor deze collimatoren, die werden geëvalueerd met digitale simulaties in HOOFDSTUK7. De kwantitatieve reconstructie van 
de multifocale collimator werd verder gevalideerd met fantoomexperimenten in HOOFDSTUK 8. De focusserende collimators verkortten de scanduur voor beide studies aanzienlijk.

Een andere methode om de interventionele scanduur te verkorten werd voorgesteld in HOOFDSTUK 9 met het evalueren van de verdeling van de radionucliden tijdens het scannen. Er werden meerdere snelle scans van elk één minuut gemaakt en de reconstructie werd na elke rotatie bijgewerkt met de nieuw verkregen data. De scan werd beëindigd wanneer de verkregen afbeeldingen voldoende stabiel waren. Digitale simulaties, fantoomexperimenten en een retrospectief patiëntonderzoek toonden aan dat de scanduur aanzienlijk kon worden verminderd, zonder verlies van diagnostische informatie.

\section{Snellere Reconstructie}

De nucleaire reconstructies moeten na het scannen snel beschikbaar zijn om ervoor te zorgen dat de kostbare tijd in de interventieruimte niet verloren gaat. De huidige geavanceerde reconstructies hebben enkele minuten nodig om te voltooien. In HOOFDSTUK 10 werd een andere methode voor reconstructie onderzocht. Deze aanpak reconstrueerde eerst de projecties met behulp van gefilterde terugprojectie en gaf de resultaten vervolgens door aan een convolutioneel neuraal netwerk voor beeldverbetering. Deze reconstructiemethode leverde beelden op met een kwaliteit die vergelijkbaar was met die van de modernste reconstructietechnieken die bovendien binnen enkele seconden werden gegenereerd. 


\section{Acknowledgments}

This dissertation would not have been possible without the help of a great number of people. I am grateful to everyone that contributed along the way and I would like to thank several people in particular.

Hugo, thank you for providing me the opportunity to work on the IXSI project and for your support over the years. I have enjoyed the freedom you gave me to pursue my interests. Your never-ending flow of ideas has been an inspiration. The research that the clinical physics group has performed over the past years may revolutionize the field of nuclear medicine. This is impressive and deserves more recognition.

Max, thank you for your contributions along the way. Your comments on manuscripts have always been helpful and your support with administrative matters was much appreciated. The Image Sciences Institute has become a world-leading institute under your supervision and provided me with an excellent scientific environment for my stay. I am grateful to be one of your final $\mathrm{PhD}$ candidates.

Marnix, thank you for your valuable clinical input throughout the project. It is impressive how you have brought so many novel treatments to the clinic in recent years. The interventional scanner has now been added to your toolbox. I trust it will be of help with your coming radionuclide therapy innovations.

Sandra and Britt, fellow IXSI PhD's, it was great to share the ups and downs in the development of the interventional scanner. Your feedback on manuscripts and assistance with phantom experiments has been invaluable. Thank you for all of your help.

Wilco, Leo, Pascal, Frank, and Maryam, the IXSI research engineers, the interventional scanner would not have been completed without your perseverance. Thank you for all of your efforts in developing prototype configurations, managing of the many involved users, and coping with the massive amounts of documentation. You can be proud of what has been achieved.

Fasco, Remco, and Daan, dear roommates, it was great to share the road to becoming a doctor. The struggles with statistics, revisions, and deadlines always felt much easier to deal with after a joint brainstorming session. Thank you for all the fun times in our office.

Arnold, Bas, Bastiaan, Casper, Edwin, Rob, and Woutjan, clinical physicists (in training) and postdocs, thank you for the valuable discussions during our meetings and lunches. I have enjoyed our trips to the Ardennen and the shared conference visits. Sander, Ahmed, and Martina, our office neighbors, thank you for the enjoyable coffee breaks. Arthur, Carin, and Margot, fellow radioembolization researchers, thank you for your input during the radioembolization meetings. 
Members of the technical cluster, thank you for your assistance concerning all computer and scanner issues. Team of the RNA-lab, thank you for supporting me with the various phantom experiments and for the many radionuclide preparations. Technicians of the nuclear medicine department, thank you for assisting me with the radioactive sources and for allowing me on the scanners so many times.

ISI and ImagO members, thank you for the organization of and the participation in the various (scientific) activities, conferences, and lunch meetings. They have been crucial in establishing my scientific frame of reference. Master, bachelor, and clinical physics students, thank you for your interest in our work and your contributions over the years.

Mom and dad, thank you for always supporting me in my studies. Jasper and Tim, dear brothers, thank you for joining me as my paranymphs on this memorable day.

And finally, Linda. This dissertation would not have been completed without your support. Thank you for everything you have done. I look forward to our future together. 


\section{List of Publications}

\section{Journal Articles}

- MMA Dietze, $S$ van der Velden, MGEH Lam, MA Viergever, HWAM de Jong. "Fast quantitative reconstruction with focusing collimators for liver SPECT”. EJNMMI Physics, 2018; 5(1):28

- $\quad S$ van der Velden, MMA Dietze, MA Viergever, HWAM de Jong. "Fast technetium-99m liver SPECT for evaluation of the pre-treatment procedure for radioembolisation dosimetry". Medical Physics, 2019; 46(1):345-355

- $\quad S$ van der Velden, B Kunnen, WJC Koppert, JHL Steenbergen, MMA Dietze, C Beijst, MA Viergever, MGE H Lam, HWAM de Jong. "A dual layer detector for simultaneous fluoroscopic and nuclear imaging”. Radiology, 2019; 290(3):833-838

- $\quad$ MMA Dietze, B Kunnen, S van der Velden, JHL Steenbergen, WJC Koppert, MA Viergever, HWAM de Jong. "Performance of a Dual-Layer Scanner for Hybrid SPECT/CBCT". Physics in Medicine and Biology, 2019; 64(10):105020

- WJC Koppert, MMA Dietze, S van der Velden, JHL Steenbergen, HWAM de Jong. "A Comparative Study of $\mathrm{NaI}(\mathrm{Tl}), \mathrm{CeBr}_{3}$, and CZT for use in a Real-Time Simultaneous Nuclear and Fluoroscopic Dual-Layer Detector". Physics in Medicine and Biology, 2019; 64(13):135012

- MMA Dietze, R Bastiaannet, B Kunnen, S van der Velden, MGEH Lam, MA Viergever, HWAM de Jong. "Respiratory Motion Compensation in Interventional Liver SPECT using Simultaneous Fluoroscopic and Nuclear Imaging”. Medical Physics, 2019; 46(8):3496-3507

- MMA Dietze, WJ Branderhorst, B Kunnen, MA Viergever, HWAM de Jong. "Accelerated SPECT Image Reconstruction with FBP and an Image Enhancement Convolutional Neural Network". EJNMMI Physics, 2019; 6(1):14

- B Kunnen, MMA Dietze, AJAT Braat, MGEH Lam, MA Viergever, HWAM de Jong. "Feasibility of imaging ${ }^{90} \mathrm{Y}$ microspheres at diagnostic activity levels for hepatic radioembolization treatment planning”. Medical Physics, 2019; 47(3):1105-1114

- MMA Dietze, B Kunnen, C Beijst, HWAM de Jong, "Adaptive Scan Duration in SPECT: Evaluation for Radioembolization”. Medical Physics, 2020; 47(5):2128-2138

- MMA Dietze, WJC Koppert, R van Rooij, HWAM de Jong, "Nuclear Imaging with an X-Ray Flat Panel Detector: A Proof-of-concept Study”. Medical Physics, 2020; 47(8)

- MMA Dietze, B Kunnen, M Stella, HWAM de Jong, "Monte Carlo-based Scatter Correction for the SMARTZOOM Collimator”. EJNMMI Physics, 2020; 7(1):49 
- $\quad$ MMA Dietze, B Kunnen, F Brontsema, P Ramaekers, C Beijst, M Afifah, AJAT Braat, MGEH Lam, HWAM de Jong, "A Compact and Mobile Hybrid C-arm Scanner for Simultaneous Nuclear and Fluoroscopic Image Guidance”. Submitted

- MMA Dietze, B Kunnen, MGEH Lam, HWAM de Jong, "Interventional Respiratory Motion Compensation by Simultaneous Fluoroscopic and Nuclear Imaging”. Submitted

\section{Conference Abstracts}

- $\quad S$ van der Velden, B Kunnen, WJC Koppert, JHL Steenbergen, MMA Dietze, C Beijst, MA Viergever, MGEH Lam, HWAM de Jong. "A dual layer detector for simultaneous fluoroscopic and nuclear imaging”. SNMMI 2017, Denver

- MMA Dietze, R Bastiaannet, MA Viergever, MGEH Lam, HWAM de Jong. "Monte Carlobased reconstruction of converging collimators and application to liver dosimetry". IEEE NSS/ MIC 2017, Atlanta

- R Bastiaannet, M Verra, MMA Dietze, MA Viergever, HWAM de Jong. "Fully Data-Driven Resiratory Motion Correction under Non-Stationary Breathing”. IEEE NSS/MIC 2017, Atlanta

- MMA Dietze, R Bastiaannet, B Kunnen, S van der Velden, MGEH Lam, MA Viergever, HWAM de Jong. "Motion Compensation in Liver SPECT using Simultaneous X-Ray and Nuclear Imaging”. RSNA 2018, Chicago

- MMA Dietze, B Kunnen, S van der Velden, JHL Steenbergen, WJC Koppert, MA Viergever, HWAM de Jong. "Performance of a Dual-Layer Scanner for Hybrid SPECT/CBCT". SNMMI 2019, Anaheim

- MMA Dietze, WJ Branderhorst, MA Viergever, HWAM de Jong. "Accelerated SPECT Image Reconstruction with a Convolutional Neural Network”. SNMMI 2019, Anaheim

- B Kunnen, MMA Dietze, AJAT Braat, MGEH Lam, MA Viergever, HWAM de Jong. "Image quality of a ${ }^{90}$ Y pretreatment procedure for radioembolisation”. EANM 2019, Barcelona

- MMA Dietze, WJC Koppert, HWAM de Jong. "High-Resolution SPECT with a Flat Panel Detector and Detailed Collimator Modeling”. IEEE NSS/MIC 2019, Manchester

- MMA Dietze, B Kunnen, HWAM de Jong. "Adaptive Scan Duration for Interventional SPECT”. IEEE NSS/MIC 2019, Manchester 



\section{Biography}

Martijn Dietze was born on the 18th of September 1993 in Capelle aan den IJssel, The Netherlands. He obtained his Bachelor's degree in Physics and Astronomy from the Utrecht University. During his Bachelor, he enrolled in an exchange year at the Australian National University and he performed his thesis partly at DESY in Hamburg. He obtained his Master's degree in Particle Physics from the University of Amsterdam. During his Master, he participated in the summer school at CERN in Geneva and he performed his thesis at Nikhef in Amsterdam. He continued as a $\mathrm{PhD}$ candidate at the Radiology and Nuclear Medicine Department of the University Medical Center in Utrecht. The results of that research are presented in this dissertation. 
